\title{
Modern topics in black hole physics and cosmology
}

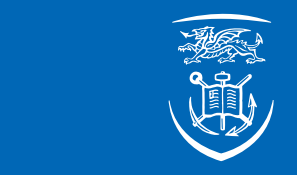

Swansea University

Prifysgol Abertawe

Francesco Filippini

Department of Physics

Swansea University

Submitted to Swansea University in fulfilment of the requirements for the degree of

Doctor of Philosophy 


\section{Declaration}

This work has not previously been accepted in substance for any degree and is not being concurrently submitted for any degree.

Signed:

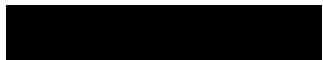
(candidate)

$01 / 05 / 2020$

Date:

\section{Statement 1}

This thesis is the result of my own investigations, except where otherwise stated. Where correction services have been used, the extent and nature of the correction is clearly marked in a footnote(s). Other sources are acknowledged by footnotes giving explicit references. A bibliography is appended.

Signed:

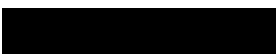
(candidate)

\section{$01 / 05 / 2020$}

Date:

\section{Statement 2}

I hereby give consent for my thesis, if accepted, to be available for photocopying and for inter-library loan, and for the title and summary to be made available to outside organisations.

Signed:

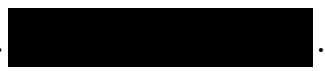
(candidate)

$01 / 05 / 2020$

Date: 



\section{Acknowledgements}

I thank my supervisor prof. Gianmassimo Tasinato for the great help and support he gave me in these years, through inspiring discussions and precious suggestions. Moreover, my thanks go to the Swansea Cosmology Group, and in particular to prof. Ivonne Zavala, for giving me the opportunity to work in a friendly and stimulating environment. Also, I want to thank prof. Carlos Nunez for insightful comments on this work.

Finally, thanks to Silvia and to my family, who have constantly encouraged and sustained me since the very first day: I dedicate this work to them. 

Considerate la vostra semenza: fatti non foste a viver come bruti, ma per seguir virtute e canoscenza. 



\begin{abstract}
Motivated by the new opportunities that gravitational wave detections offer in both cosmology and astrophysics, in this thesis we study potentially detectable physical phenomena related with black hole physics, with particular interest to systems of black holes interacting with cosmological scalar fields.
\end{abstract}

First of all, we study a charged, rotating black hole solution in a Generalised Proca theory of gravity, obtained through a disformal transformation of the Einstein-Maxwell action. Due to the breaking of gauge symmetry, the gauge freedom of the components of the gauge vector is lost; thus, for a particular choice of the gauge vector, we are able to find an exact, charged, rotating black hole solution, with event horizon and ergosphere parametrically different from the General Relativity solution. For such solution, the Innermost Stable Circular Orbits (ISCOs) are studied. Subsequently, we consider the effects of an external, ultralight scalar field interacting with an electromagnetic Kerr-Newman black hole. Depending on the frequencies of the scalar's modes and on the angular velocity of the black hole, the scalar waves scattering off the event horizon may trigger superradiance; for some scalar field's modes, a stationary scalar cloud is developed in the black hole's surroundings, and the frequencies of the scalar's modes in the cloud correspond to the resonant frequencies of the superradiant gain factor. Moreover, we investigate the effects of a massless scalar field coupled to an electromagnetic rotating black hole through Chern-Simons couplings; in particular, we consider both scalar-vector and scalar-tensor couplings. Using both a slow rotation and small couplings approximation scheme, we are able to find an hairy black hole solution up to second order in the approximation parameters. Such solution carries a secondary scalar charge, and it satisfies the Smarr formula for black hole thermodynamics. Furthermore, we propose a possible revealing effect for such hairy configuration, based on the polarization dependent angular deflection to which photons are subject to when interacting with the scalar field. At the end, we study the linear perturbation theory for a static, electromagnetic black hole, also including a Chern-Simons scalar-vector coupling to an external, massless axion field. We find that due to the presence of the magnetic charge the decoupling between axial and polar perturbations is broken, leading to a Regge-Wheeler perturbation equation containing terms with different parity. 


\section{Contents}

List of Figures

1 Introduction and theory review

1.1 Introduction . . . . . . . . . . . . . . . . . . . ⿴囗十

1.2 Black holes part 1: a minimal review . . . . . . . . . .

1.2.1 The Schwarzschild solution . . . . . . . . . . . प प

1.2.2 Charged case: the Reissner-Nordström solution . . . . . . . ए2

1.2.3 Rotating case: Kerr and Kerr-Newman solution . . . . . . . प् प5

1.2.4 Black hole thermodynamics ... . . . . . . . . 20 201

1.3 Modified Gravity: introduction . . . . . . . . . . . . . . . . . . .

1.3.1 Beyond General Relativity . . . . . . . . . . . . ए2]

1.3.2 Modified gravity theories: a roadmap . . . . . . . . . 25

1.3.3 Tests of gravity theories . . . . . . . . . . . . B4

1.4 Axion cosmology: the basics . . . . . . . . . . . . . . 35

1.5 Superradiance in a nutshell . . . . . . . . . . . . . . . . 40

1.6 Black holes part 2: no-hair theorem . . . . . . . . . . . . . 4.3

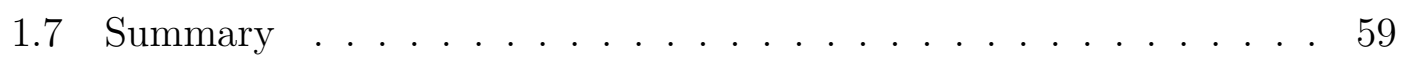

2 A new rotating black hole solution 67

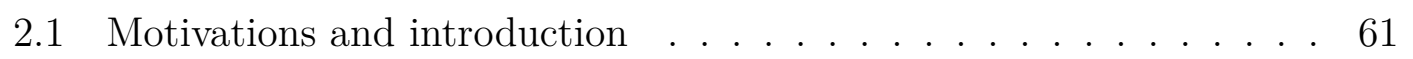

2.2 Set-up: disformal transformation of a Kerr-Newman black hole . . . 64

2.3 Singularities, Killing vectors and horizons . . . . . . . . . . . . r r

2.4 New rotating solutions in vector-tensor theories of gravity . . . . . . [3]

2.4.1 Case 1: $A_{r}(r, \theta)=A_{\theta}(r, \theta)=0 \ldots \ldots$. . . . . . . . . .

2.4.1.1 Horizons and singularities ......... [ 
2.4.2 Case 2: $A_{r}(r), A_{\theta}(r, \theta)=0 \ldots \ldots$. . . . . . . . . .

2.4.2.1 Structure and properties of horizons . . . . . . 82

2.4.2.2 Maximal black hole spin and horizon oblateness . . 8.5

2.4.2.3 Equatorial orbits . . . . . . . . . . 89

2.4.3 Case $3: A_{r}(r), A_{\theta}(\theta) \ldots \ldots \ldots$

2.4.4 Case $4: A_{r}(r, \theta), A_{\theta}(r, \theta) \ldots \ldots \ldots$

2.5 Discussion . . . . . . . . . . . . . . . . . . ए एण2

2.6 Appendix: mass, angular momentum and charge . . . . . . . . . . 104

3 Scalar Clouds 107

3.1 Introduction and motivations . . . . . . . . . . . . . एण

3.2 Scalar field clouds . . . . . . . . . . . . . . . Ш3

3.2.1 Asymptotic region . . . . . . . . . . . . . . एप

3.2 .2 Near region . . . . . . . . . . . . . . . . . ए20

3.2.2.1 Extremal case: $\tau=0 \ldots \ldots$. . . . . . . . . .

3.2.2.2 General case: $\tau \neq 0 \ldots \ldots$. . . . . . . . . .

3.3 Conclusions . . . . . . . . . . . . . . . . . . . . . . . . . . . . . ए ए22

4 Black hole hair [3]

4.1 Introduction . . . . . . . . . . . . . . . . एउप

4.2 System under consideration . . . . . . . . . . . . . . ए34

4.2 .1 The action . . . . . . . . . . . . . . . . . . . . . . .

4.2.2 Long range axion hair and black holes . . . . . . . . . . ए38

4.3 Spherically symmetric configurations . . . . . . . . . . . . . . . प40

4.3.1 A Smarr formula for our configurations . . . . . . . . . . . . 144

4.3.2 An exact solution for large values of $\lambda \ldots \ldots$. . . . . . 5 .50

4.4 Slowly rotating charged configurations . . . . . . . . . . . ए552

4.5 Phenomenological considerations . . . . . . . . . . . ए56

4.5.1 Black hole axion hair and light polarisation . . . . . . . . ए56]

$4.5 .2 \quad$ ISCOs . . . . . . . . . . . . . . . . . . . . . . . . . . . . . .

4.5.3 Scalar hair in Active Galactic Nuclei . . . . . . . . . . . ए67

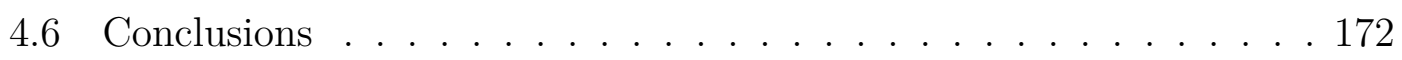


5 Black hole perturbations

5.1 Introduction and motivations . . . . . . . . . . . . . . ए75

5.2 Set-up . . . . . . . . . . . . . . . . . . . . 185

5.3 Regge-Wheeler gauge . . . . . . . . . . . . . . . . . . . ए87

5.3 .1 Schwarzschild case . . . . . . . . . . . . . . . एप्प

5.4 Electric Reissner-Nordström case . . . . . . . . . . . . . . . . ए ए97

5.5 Magnetic Reissner-Nordström case . . . . . . . . . . . . . . . 203

5.6 Electric Chern-Simons case . . . . . . . . . . . . . . . . . ए ए06

5.7 Magnetic Chern-Simons case . . . . . . . . . . . . . . . . ए 20

5.8 Conclusions . . . . . . . . . . . . . . . . . . . 215

6 Conclusions

References $[22]$ 


\section{List of Figures}

1.1 Horizons, ergosurfaces and singularity for a Kerr black hole, with $a<M$. Picture from "Schwarzschild and Kerr Solutions of Einsteins Field Equation: an introduction", by C. Heinicke and F. W. Hehl [1]]. . . . . . . . . . . . . . . [प]

1.2 Horizons, ergosurfaces and singularity for an extremal Kerr black hole, with $a=$ M. Picture from "Schwarzschild and Kerr Solutions of Einsteins Field Equation: an introduction", by C. Heinicke and F. W. Hehl [1]]. . . . . . . . . . . . . . . एप

1.3 Picture "Content of the Universe Pie Chart", from Wilkinson Microwave Anisotropy Probe (WMAP) , National Aeronautics and Space Administration. Since the WMAP data is accurate to two digits, the total of these numbers is not 100\%. . . . . . . . . Z

1.4 The diagram, taken from [0], illustrates the different ways to circumvent the Lovelock's theorem by relaxing any of its assumptions, ending up with different alternative theories of gravity. . . . . . . .

2.1 Pictorial representation of the shape of horizon and ergosphere for the disformal black hole using Boyer-Lindquist coordinates, as discussed in the main text. 


\section{LIST OF FIGURES}

2.2 Panel on the left: Innermost circular orbits for a Kerr black hole: radial position of the orbit $x=\bar{r} / M$ versus black hole's spin $a / M$. There are two branches of solutions, corresponding to corotating (black) and counterrotating (blue) ISCOs. The red line corresponds to the horizon, and the shaded part the forbidden region inside the black hole horizon. The green line is the boundary of the ergosphere. Panel on the right: binding energy $1-E$ versus black hole spin a/M. 95

2.3 Innermost circular orbits (panel on the left) and binding energy versus black hole's spin (panel on the right) for a black hole with $q=-0.2$. Color codes as in Figure [.g. The corotating ISCO does not touch the black hole's horizon, even for extremal values of the

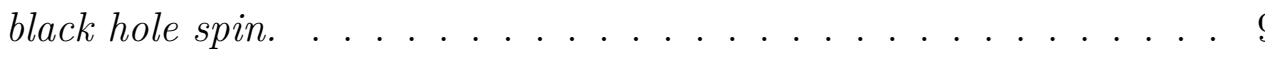

2.4 ISCOs (left) and binding energies (right) versus black hole's spin, for a black hole with $q=-5$. Color codes as in Figure [2.9. The corotating ISCO neither touches the black hole's horizon nor the ergosphere, even for extremal values of the black hole's spin. . . . .

3.1 Effective potential for a Schwarzschild black hole in the case $M \mu=$ 0.5 , with $M$ and $\mu$ being the black hole's mass and scalar field's mass respectively. On the plot, the radial coordinate $r / M=2$ corresponds to the Schwarzschild radius $r=2 M$, where the event horizon is located. $\square$

3.2 Effective potential for a Kerr black hole in the case $M \mu=0.5$, with $M$ and $\mu$ being the black hole's mass and scalar field's mass respectively. On the radial axis, the plot is truncated at the event horizon's location. . . . . . . . . . . . . . . . . . . . प8

4.1 Force-Free plasma (grey area) surrounding the black hole (black area): inside the force-free area, the electric field is screened by the plasma, which acts as a perfect conductor. Thus, inside the force-free area there is no source for the scalar field (blue line), and no hair is developed. Instead, outside the grey area there is an unscreened, non vanishing electric field which can act as a source for the scalar field through a Chern-Simons coupling term. So, the black hole develops a scalar hair outside the force-free area. 
4.2 The force-free plasma (grey area) is effective from an effective screening distance $r_{e}$. In the region enclosed between the event horizon and the force-free area $\left(r_{H} \leq r \leq r_{e}\right)$ the electric field is non vanishing, hence sourcing a scalar hair (blue line) surrounding the black hole (black area). Inside the force-free region, the plasma totally screens the electric field, and consequently the Chern-Simons term proportional to $\vec{E} \cdot \vec{B}$ vanishes, screening the black hole's scalar hair.

5.1 The gravitational wave signal of GW150914. Image from [3]. . . . ए ए78

5.2 The picture shows how the theoretical Gravitational Wave signal is obtained for the different stages of the collapse of two rotating black holes into a single one: PN (Post-Newtonian) theory for the inspiral phase (blue region), numerical relativity for the merger phase (green region), black hole perturbation theory for the ringdown (red area). Image from the talk Gravitational Waves: a new window to explore the Universe by Philippe Jetzer at Joint annual meeting of the Swiss and Austrian Physical society in 2017 . . . . . . . ए78

5.3 Schematic diagram of the axial sector for metric, vector and scalar perturbations. The axial metric's perturbations $W(t, r)$ are sourced by the vector ones $a_{B}(t, r)$, which are sourced by the scalar field perturbations $\delta \phi(t, r)$. Therefore, although the link is not explicitly manifested from the equations, the metric's perturbations implicitly depend on the scalar ones. In the picture, the solid black lines denotes the "direct" dependencies, while the dashed one denotes the "implicit" dependency metric/scalar.

5.4 Schematic diagram of the mixed metric, vector and scalar perturbations. The axial metric's perturbations $W(t, r)$ are sourced by the polar vector ones $a_{0}(t, r), a_{R}(t, r), a_{E}(t, r)$, which are sourced by the scalar field perturbations $\delta \phi(t, r)$. Although not explicitly, the metric's perturbations implicitly depend on the scalar ones through the polar vector perturbations. The solid black lines denotes the "direct" dependencies, while the dashed one denotes the "implicit" one. . . . 저 


\section{Chapter 1}

\section{Introduction and theory review}

\subsection{Introduction}

In 2015, exactly one hundred years after its publication, Einstein's General Relativity (GR) [4] had one more outstanding confirmation of its predictions: for the first time, we were able to directly detect gravitational waves coming from a binary black hole system, namely a couple of black holes rapidly spinning one around the other and finally merging into a single one [5]. This gravitational wave event, now known as GW150914 (Gravitational-Waves-15-09-14, since the detection was on the $14^{\text {th }}$ of September), marked the beginning of a new era of both cosmology and astrophysics; in fact, for the first time we had direct access to strong gravity regime observations, with the opportunity to test once more what Einstein's gravitational theory had predicted one century ago. But if it was just for tests of General Relativity, GW150914 would have been just one of the many confirmations of Einstein's theory in the last years. The reason why GW150914 must be considered a milestone event is due to the fact that it has opened the doors to direct tests of strong gravity regime; in particular, since detectable gravitational waves are likely to come from black holes, such detections provide a tool to test both black holes existence and the viability of gravity theories alternative to General Relativity. In fact, during the last 30 years, most research in cosmology has focused on the necessity of solving the two biggest cosmological problems we still have, namely dark energy and dark matter [ㅁ, [7]; the former describes the mys- 


\section{INTRODUCTION AND THEORY REVIEW}

terious and unpredicted accelerated expansion of the Universe, while the latter is related with the amount of matter we are not directly observing in the Universe. Since dark matter and dark energy together constitute more than the 95\% of the composition of the Universe, they currently are absolute priorities in the cosmological research. About the dark energy problem, in the last decades much effort has been put in trying to find some gravity theories alternative to General Relativity which could be able to both predict Einstein's confirmed predictions and at the same time to solve the dark energy problem. Thus, such modified gravity theories $[8,9]$ must somehow agree with General Relativity predictions at low energy scale (for example the Solar System one), where gravity is in a weak field regime; therefore, the strong gravity regime represents the border between General Relativity and the modified gravity theories, namely it is the regime where different theories manifest distinctive features and predictions. For this reason, GW150914 can be considered a referee of modified gravity theories, since it directly provides physical constraints to test the validity of such theories. Remarkably, in the last five years many gravitational wave detections have been confirmed, making them a reliable and efficient tool to test theories with experiments. In particular, although gravitational waves have been detected also coming from the merger of neutron stars, black holes are the best candidates for the emission of detectable gravitational waves, making them excellent open sky laboratories to test gravity theories. For this reason, it is important to study black holes in alternative theories of gravity, and how gravitational waves are generated and propagate. Moreover, it is also important to study physical systems which could produce distinctive phenomena to be tested with observations, in order to set some constraints on the parameters of the theory.

This is exactly the main goal of this thesis. In particular, we have focused our research on physical systems where black holes interact with scalar fields, since they are well motivated by string theory and particle physics and they are good candidates for solving both the dark energy and the dark matter problems.

This thesis is organised as follows. The first chapter, which includes this introduction, contains the basic theory review necessary for understanding the topics to come. The theory sections are thought to be as self consistent as possible, and 
for more exhaustive discussions external references are provided. The remaining chapters constitute the core of this thesis, since they contain the results on which my $\mathrm{PhD}$ research has been based on and the authentic and original results we have achieved; in both cases, references are provided.

In chapter $\rrbracket$, we study possible black hole solutions in a Generalised Proca theory of gravity [10, 11], obtained with an ad hoc disformal transformation [12, [13] of the Einstein-Maxwell action, using as disformal vector the $U(1)$ Maxwell one. The disformed action is not manifestly gauge invariant: due to the breaking of gauge symmetry, the gauge freedom of the components of the Maxwell gauge vector is lost; thus, depending on the components of the gauge vector, regular or singular solutions appear. For a particular choice of the gauge vector, we are able to find an exact, charged, rotating black hole solution, with event horizon and ergosphere parametrically different from the General Relativity (Kerr-Newman) solution. For such solution, we study the Innermost Stable Circular Orbits (ISCOs), which manifest different binding energies compared to the GR ones.

In chapter B, we consider the effects of an external, ultralight scalar field interacting with an electromagnetic Kerr-Newman black hole. As we will see, the presence of both electric and magnetic charges can lead to interesting effects. Depending on the frequencies of the scalar's modes and on the angular velocity of the black hole, the scalar waves scattering off the event horizon may trigger superradiance, namely being enhanced due to scattering and therefore being reflected with more energy than the incident one. The "boost" of energy is extracted from the black hole: in this case, rotational energy is extracted, and the black hole slows down. Interestingly, for some scalar field's modes a stationary scalar cloud is developed in the black hole's surroundings; such modes are quasi normal, namely their frequencies are almost purely real, and thus they are extremely long lived. As it happens in the electric case, the frequencies associated to the scalar cloud's modes are completely determined by the black holes parameters (mass, angular momentum, charge) and by the scalar field's mass. 


\section{INTRODUCTION AND THEORY REVIEW}

In chapter $⿴$, we analyze the effects of a massless scalar field coupled to an electromagnetic static/rotating black hole. In particular, we consider both scalarvector [14] and scalar-tensor Chern-Simons couplings [15, 16]. In the static case, the scalar-tensor Chern-Simons coupling vanishes, but if both the electric and the magnetic charges are on, then a scalar hair is naturally developed, being completely determined by the Chern-Simons coupling constant and the black hole's parameters; in this sense, such scalar hair is called "secondary". Moreover, using both a slow rotation and small couplings approximation scheme we study the rotating case, with both scalar-vector and scalar-tensor Chern-Simons couplings being on; up to second order in the approximation parameters, we find an hairy black hole solution depending on both the Chern-Simons couplings; again, the scalar charge is secondary. For such solutions we verify that they satisfy the thermodynamics Smarr formula [17]. Furthermore, due to the coupling between the scalar field and the electromagnetic vector, we propose a possible revealing and detectable effect for such hairy configuration, based on the polarization dependent angular deflection to which photons are subject to when interacting with the scalar field around the black hole. Moreover, we briefly discuss about hairy black holes in Active Galactic Nuclei (AGNs).

In chapter [1, we study the linear perturbation theory for an electromagnetic, static black hole, since we are interested in studying perturbations arising from both electric and magnetic charges. Working in the Regge-Wheleer gauge [18] and decomposing the perturbations on a tensor spherical harmonics basis, according to their angular behaviour we can divide perturbations into two sectors: odd parity (axial) perturbations and even parity (polar) ones. Interestingly, unlike what happens in the Schwarzschild and in the electric Reissner-Nordström case, we find that in the magnetic Reissner-Nordström case it is not possible to completely decouple axial and polar perturbations. In fact, focussing on the axial part and writing the Regge-Wheleer equation for the metric's and the vector's perturbations, we find the presence of both parity odd and parity even terms. Moreover, we also study the linear perturbations theory for an electromagnetic, static black hole coupled to an external, massless scalar field through a Chern-Simons scalar-vector term. Again, we find that due to the presence of the magnetic charge the decoupling 
between axial and polar perturbations is broken, leading to Regge-Wheeler equations involving terms with different parity.

Finally, chapter 6 contains a brief summary of the main results discussed and some ideas for further investigations.

\subsection{Black holes part 1: a minimal review}

In this section, we introduce some basic but necessary concepts about black hole physics we will need in the following chapters: for more details, see exhaustive reviews [1, [19-21] or textbooks [22-27]. Throughout this work, we use a mostly plus $(1,3)$ metric signature, and all quantities are expressed in natural units $c=\hbar=G=1$, with the additional choice $4 \pi \epsilon_{0}=1$.

In 1915, Einstein published one of the most outstanding theoretical result in modern physics, i.e. the theory of General Relativity (GR) [4, [28]. GR is not merely an upgrade or generalisation of the previous gravitation theory belonging to Newton, but it is rather a completely new approach to gravity. In fact, gravity in GR is treated as a geometrical theory, with the related complex but powerful formalism. Being the spacetime described by a manifold, the basic object of GR is the metric tensor $g_{\mu \nu}$, namely a covariant, second-degree, non degenerate, symmetric tensor with signature $(1,3)$, which encodes and describes the geometric and causal structure of the spacetime; for more details or more accurate formalism, the

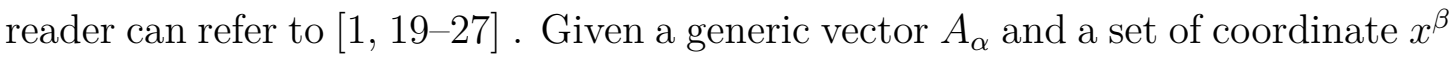
on the manifold, on each metric there is a naturally defined covariant derivative

$$
\nabla_{\alpha} A_{\beta}=\frac{\partial A_{\beta}}{\partial x^{\alpha}}-\Gamma_{\alpha \beta}^{\lambda} A_{\lambda}, \quad \nabla_{\alpha} A^{\beta}=\frac{\partial A^{\beta}}{\partial x^{\alpha}}+\Gamma_{\alpha \lambda}^{\beta} A^{\lambda},
$$

with $\Gamma_{\alpha \beta}^{\lambda}$ being the Christoffel symbols defined as

$$
\Gamma_{\alpha \beta}^{\lambda}=\frac{1}{2} g^{\rho \lambda}\left(\frac{\partial g_{\alpha \rho}}{\partial x^{\beta}}+\frac{\partial g_{\beta \rho}}{\partial x^{\alpha}}-\frac{\partial g_{\alpha \beta}}{\partial x^{\rho}}\right)
$$


The easiest example of spacetime is the flat one, known as Minkowski spacetime, which is described by the diagonal metric

$$
g_{\mu \nu}=\operatorname{diag}(-1,1,1,1)
$$

whose associated covariant derivative is simply the usual derivative.

One of the key concept of GR is the spacetime curvature, describing how and how much the spacetime is curved compared with the flat one. Formally, we define the Riemann curvature tensor as

$$
R_{\beta \gamma \delta}^{\alpha}=\partial_{\gamma} \Gamma_{\beta \delta}^{\alpha}-\partial_{\delta} \Gamma_{\beta \gamma}^{\alpha}+\Gamma_{\beta \delta}^{\mu} \Gamma_{\mu \gamma}^{\alpha}-\Gamma_{\beta \gamma}^{\mu} \Gamma_{\mu \delta}^{\alpha}
$$

and we furthermore define the Ricci tensor

$$
R_{\mu \nu}=R_{\mu \lambda \nu}^{\lambda}
$$

and the Ricci scalar

$$
R=g^{\mu \nu} R_{\mu \nu}
$$

For a flat spacetime, we have

$$
R=0 \text {. }
$$

However, as we will see, $R=0$ is not a sufficient condition to have a flat spacetime, since the condition is satisfied even for curved but "Ricci traceless" spacetimes.

Up to now, we have just been giving a geometric description of spacetimes, defining geometrical objects to describe geometric features of the spacetime itself. However, what makes GR such a remarkable theory is what is encoded in its most famous and powerful result, Einstein's equation:

$$
R_{\mu \nu}-\frac{1}{2} g_{\mu \nu} R=T_{\mu \nu}
$$

where $R_{\mu \nu}$ and $R$ are the Ricci tensor and the Ricci scalar respectively and $T_{\mu \nu}$ is the energy-momentum tensor (or stress-energy tensor), which describes the content and the behaviour of energy and matter in the spacetime. ${ }^{\text {. }}$ The extraordi-

\footnotetext{
${ }^{1}$ Remember that we are using natural units; in physical units, Einstein's equations reads: $R_{\mu \nu}-\frac{1}{2} g_{\mu \nu} R=\frac{8 \pi G}{c^{4}} T_{\mu \nu}$.
} 
nariness of the Einstein's Equation (‥8) is based on the fact that its left-hand side (LHS) only contains geometrical objects, while on the right-hand side (RHS) there is an object encoding informations on the energy/matter content of the spacetime; hence, the equality implies that the spacetime geometry depends on the matter/energy content, but at the same time the behaviour of such energy/matter depends on the geometry. Quoting A. Wheeler: "Space tells matter how to move, matter tells space how to curve".

Solving the Einstein's Equation (피) means finding the 10 components of the metric tensor $g_{\mu \nu}$ which satisfies the equation and subject to covariant conservation constraints; technically, this problem consists in solving a set of 10 non-linear, partial differential equations, which in most cases is impossible. However, for some particular highly symmetric cases, it happens that the Einstein's Equation is fully analytically solvable.

\subsubsection{The Schwarzschild solution}

To start, we choose to solve the equation ([.8) in vacuum, namely $T_{\mu \nu}=0$, which leads to

$$
R_{\mu \nu}-\frac{1}{2} g_{\mu \nu} R=0
$$

As one would expect, the Minkowski spacetime is a solution of (एप्) ; in addition, under specific assumptions, it is possible to find other interesting solutions. One of the most common choices is to assume spherical symmetry, since (to a good approximation) that is the case for many astrophysical systems like the gravitational field of celestial bodies. In GR, as stated in the Birkhoff theorem (see for example [22] for more details), the unique spherically symmetric vacuum solution of the Einstein's Equation is the Schwarzschild metric [2.9], named after the German physicist Karl Schwarzschild who derived it in 1916. Using a Boyer-Lindquist spherical coordinate system $\{t, r, \theta, \varphi\}$, the Schwarzschild metric reads:

$$
d s^{2}=-\left(1-\frac{2 M}{r}\right) d t^{2}+\left(1-\frac{2 M}{r}\right)^{-1} d r^{2}+r^{2} d \theta^{2}+r^{2} \sin ^{2} \theta d \varphi^{2},
$$


where the constant $M$ is interpreted as the gravitational mass ${ }^{m}$ of the gravitating object. The Schwarzschild metric is a static, asymptotically flat and spherically symmetric solution of the Einstein's Equation.

Roughly speaking, being static means that the metric does not explicitly depend on time and that it is invariant under time reversal $(t \rightarrow-t)$ transformation. With a bit more formalism, a metric is called static if it admits a Killing vector which is timelike at spatial infinity and orthogonal to $t=$ const hypersurfaces.

Asymptotically flatness implies that at spatial infinity the metric reduces to the Minkowsky one, since at infinitely large distances from the source $M$, the spacetime curvature is negligible (for a more formal definition, see for example [26]). Finally, spherical symmetry is satisfied if and only if the following commutation relations are satisfied:

$$
[R, S]=T, \quad[S, T]=R, \quad[T, R]=S
$$

with $(R, S, T)$ being the Killing vectors of the 2 -sphere $S^{2}$ given in coordinate basis $(\theta, \varphi)$ by:

$$
\begin{aligned}
R & =\partial_{\varphi} \\
S & =\cos \varphi \partial_{\theta}-\cot \theta \sin \varphi \partial_{\varphi} \\
T & =-\sin \varphi \partial_{\theta}-\cot \theta \cos \varphi \partial_{\varphi} .
\end{aligned}
$$

What makes the Schwarzschild metric really remarkable and fascinating are its simplicity and depth. In fact, despite being described by a simple expression, the metric (미) hides some intriguing features and phenomena which still have the attention of physicists from very different research areas.

If we look at (․ㅣ), we notice that the metric becomes singular at two points:

$$
r=0, \quad 1-\frac{2 M}{r}=0 \Longrightarrow r_{S}=2 M .
$$

However, even though they both lead to divergences of the metric (ㄸ. $\mathbb{0}$ ), they have a completely different nature.

\footnotetext{
${ }^{1}$ We do not motivated it in this chapter, since a quick derivation is shown in the appendix at the end of the next chapter.
} 
In fact, as we will show soon, only $r=0$ is a physical singularity, while $r_{S}$, known as Schwarzschild radius, is just a coordinate singularity, which can be therefore removed by a suitable change of coordinates. To understand it, let's first focus on the $r=0$ singularity.

In GR, a singularity could be simply ${ }^{\mathbb{m}}$ defined as a set of points (or even a single one) where the spacetime curvature tends to infinity. Hence, to check if a geometry has any singularity, it is necessary to study the curvature invariants $\left(R, R^{\mu \nu} R_{\mu \nu}\right.$, $\left.R^{\mu \nu \alpha \beta} R_{\mu \nu \alpha \beta}\right)$. For the Schwarzschild metric, we get:

$$
R=0, \quad R^{\mu \nu} R_{\mu \nu}=0, \quad R^{\mu \nu \alpha \beta} R_{\mu \nu \alpha \beta}=\frac{48 M^{2}}{r^{6}}
$$

First of all, we notice that both the Ricci scalar and the contracted Ricci tensor $\left(R^{\mu \nu} R_{\mu \nu}\right)$ are everywhere null; however, since the Kretschmann scalar ( $K=$ $\left.R^{\mu \nu \alpha \beta} R_{\mu \nu \alpha \beta}\right)$ does not vanish, the geometry is not flat. From the Kretschmann scalar, we notice that it diverges at locus $r=0$, which is therefore a singularity by definition, while there is no divergence at point $r=r_{S}$.

So, what happens at $r=r_{S}$ ? From the expression of the Schwarzschild metric, we notice that at $r_{S}$ the metric component $g_{r r}$ diverges, which implies that the inverse metric component $g^{r r}$ vanishes at that point. In particular, we have $g^{r r}>0$ for $r>r_{S}$ and $g^{r r}<0$ for $r<r_{S}$. This means that the vector $\partial_{\mu} r$ changes from being spacelike to be timelike at surface $r_{S}$ (where it is a null vector), since by definition we have

$$
g^{r r}=g^{\mu \nu} \partial_{\mu} r \partial_{\nu} r
$$

In addition, since we have

$$
g^{t t}=0 \text { if } r=r_{S}, \quad g^{t t}<0 \text { if } r>r_{S}, \quad g^{t t}>0 \text { if } r<r_{S},
$$

we observe that inside the Schwarzschild radius the time coordinate " $t$ " and the radial coordinate " $r$ " swap their sign, and hence their nature. So, if we consider a a photon travelling with velocity purely along the radial direction, once it crosses the Schwarzschild radius it cannot reverse its motion and crossing it back, since

\footnotetext{
${ }^{1}$ Again, we want to be as simpler as we can, using intuitive definition for geometrical objects which could be rather defined with much more formality.
} 


\section{INTRODUCTION AND THEORY REVIEW}

its future lightcone is now oriented towards the singularity [24], having both the time coordinate and the radial one changed their signs. Therefore, any photon inside the Schwarzschild horizon cannot escape from it, and consequently no signal can be transmitted from the region inside the Schwarzschild radius. We define the surface $r_{S}$ to be an event horizon, since it is the boundary of two causally disconnected spacetime regions where events outside the horizon cannot be influenced by anything from the inside. (For a more rigorous and formal definition, see for example [25-[27]). The event horizon and the inner singularity make the Schwarzschild solution a black hole solution, to indicate that there exists a spacetime region from where signals (and hence light) cannot escape. Remarkably, it has been conjectured by Penrose [30] that singularities cannot be "naked", i.e. they must be contained and protected by a horizon: so, if a spacetime has any singularity, it necessary has a horizon as well.

Other interesting features of the Schwarzschild black hole solution are its stable orbits. In fact, we can imagine a photon or a test mass orbiting around a Schwarzschild black hole: for specific values of energy/mass or angular momentum, it is possible to have the test particle orbiting around the black hole in a stable orbit, with the gravitational attraction acting as a centripetal force. These orbits are quite important for astrophysical systems since they are the last close orbits where particle can stably rotate.

In the case of photons, the last stable orbit represents the photon circle, which due to spherical symmetry is actually a photon sphere: if we consider an astrophysical black hole surrounded by gas and plasma, the photon sphere together with gravitational lensing are responsible of the black hole shadow at the center of the observable bright disk, as remarkably captured and shown in [31-36] for the supermassive black hole on the center of galaxy M87.

For a massive particle, instead, the Innermost Stable Circular Orbit (ISCO) is the closest, stable orbit for a binary gravitating system, such as binary black holes: so, if we consider a binary system of merging black holes like the ones observed with gravitational waves detection [37], the ISCO determines the frequency of the emitted gravitational waves before the merger happens. 
Since we will use it in the next chapters, let's now quickly see how the ISCO trajectories are computed.

In a spherically symmetric spacetime, it is convenient to choose the equatorial plane $(\theta=\pi / 2$ in a Boyer-Lindquist coordinate system) as the reference one; on that plane, the Schwarzschild metric reads

$$
d s^{2}=-\left(1-\frac{2 M}{r}\right) d t^{2}+\left(1-\frac{2 M}{r}\right)^{-1} d r^{2}+r^{2}\left(d \theta^{2}+d \phi^{2}\right)
$$

Considering a generic particle approaching the black hole on the equatorial plane, its 4 -velocity is

$$
u^{\mu}=\left\{u^{t}, u^{r}, u^{\theta}, u^{\phi}\right\}=\left\{\frac{d t}{d \tau}, \frac{d r}{d \tau}, \frac{d \theta}{d \tau}, \frac{d \phi}{d \tau}\right\}
$$

with $\tau$ being the proper time. However, since the motion is restrained on the plane, the velocity's component along the $\theta$ direction is set to zero:

$$
u^{\theta}=0
$$

Considering massive/massless particles respectively, the 4-velocity satisfies the condition

$$
u_{\mu} u^{\mu}=-1, \quad u_{\mu} u^{\mu}=0 .
$$

With the 4-velocity, using the metric (ㅁ) we can also define the conserved energy $\mathbf{E}$ and the conserved angular momentum $\mathbf{L}$ respectively as

$$
\begin{aligned}
& E=-\left(g_{t t} u^{t}+g_{t \phi} u^{\phi}\right), \\
& L=g_{\phi t} u^{t}+g_{\phi \phi} u^{\phi} .
\end{aligned}
$$

Using the definitions above, considering a massive particle the relation ([.20) reads

$$
\left(\frac{d r}{d \tau}\right)^{2}-E^{2}+\left(1-\frac{2 M}{r}\right)\left(1+\frac{L^{2}}{r^{2}}\right)=0
$$




\section{INTRODUCTION AND THEORY REVIEW}

which can be recast as

$$
\frac{1}{2}\left(\frac{d r}{d \tau}\right)^{2}+V_{\text {eff }}=\frac{E^{2}-1}{2}
$$

where we have introduced the effective potential

$$
V_{e f f}=-\frac{M}{r}+\frac{L^{2}}{2 r^{2}}-\frac{M L^{2}}{r^{3}}
$$

We are now interested to find the explicit trajectory of the ISCO; first of all, being the trajectory circular the radial velocity must vanish:

$$
\frac{d r}{d \tau}=0 \rightarrow V_{e f f}=\frac{E^{2}-1}{2}
$$

Moreover, for the motion to be circular the acceleration must vanish as well:

$$
\frac{d^{2} r}{d \tau^{2}}=0 \rightarrow \frac{\partial V_{e f f}}{\partial r}=0
$$

Finally, for the trajectory to be stable the effective potential has to be at a local minimum:

$$
\frac{\partial^{2} V_{e f f}}{\partial r^{2}}=0
$$

Equations (ए.25, ए.26], ए.27) can be solved to get the values for $E, L, r_{I S C O}$ which determine the innermost stable circular orbit for the Schwarzschild geometry.

For a massive particle, the ISCO is located at

$$
r_{I S C O}=6 M=3 r_{S}
$$

while for massless particles the ISCO is located at

$$
r_{I S C O}=3 M=\frac{3}{2} r_{S}
$$

\subsubsection{Charged case: the Reissner-Nordström solution}

As we have seen, the Schwarzschild black hole solution describes the vacuum spacetime around a gravitating mass $M$, where nothing else than the mass itself gener- 
ates the geometry: so, at this point, it could be interesting to see what happens if extra matter/radiation is taken into account. In particular, to keep things simple but interesting, we now consider the case of a gravitating mass $M$ together with an electromagnetic field, neglecting the presence of extra matter. In natural units, such system can be described by the following action

$$
S=\int d^{4} x \sqrt{-g}\left[\frac{R}{4}-\frac{1}{4} F^{\mu \nu} F_{\mu \nu}\right]
$$

with $F_{\mu \nu}$ being the electromagnetic field strength given by

$$
F_{\mu \nu}=\partial_{\mu} A_{\nu}-\partial_{\nu} A_{\mu}
$$

Since the action (1.30) involves the Ricci scalar and the standard electromagnetic field strength only, it is usually referred to as the Einstein-Maxwell action. Now, due to the presence of the electromagnetic field strength in the action, we have to solve two different equations of motion, one for the metric tensor and one for the gauge vector, respectively. Hence we have:

$$
\begin{aligned}
& R_{\mu \nu}-\frac{1}{2} R g_{\mu \nu}=2 T_{\mu \nu}, \\
& \nabla_{\mu} F_{\nu}^{\mu}=0
\end{aligned}
$$

where the electromagnetic energy-stress tensor takes the form

$$
T_{\mu \nu}=F_{\mu}^{\alpha} F_{\nu \alpha}-\frac{1}{4} g_{\mu \nu} F^{\alpha \beta} F_{\alpha \beta}
$$

Without any further assumption or simplification, solving the above set of equations can be very hard, or even (as it often happens to be) impossible. However, as in the Schwarzschild case, an incredibly simple but no less interesting solution can be found if we make the assumption of spherical symmetry, where the spacetime metric can be written as

$$
d s^{2}=A(t, r) d t^{2}+B(t, r) d r^{2}+d \Omega^{2}
$$




\section{INTRODUCTION AND THEORY REVIEW}

with $d \Omega^{2}$ being the 2 -sphere metric

$$
d \Omega^{2}=r^{2} d \theta^{2}+r^{2} \sin ^{2} \theta d \varphi^{2}
$$

Moreover, we also assume the background to be static, which leads to

$$
A(t, r) \rightarrow A(r), \quad B(t, r) \rightarrow B(r) .
$$

Furthermore, we are interested in asymptotically flat solutions, i.e. solutions which approach the vacuum Minkowski solution as the distance from the body approaches infinity.

The first "black hole solution" to this problem was found back in the years 19161918 thanks to the work done by Hans Reissner [38] and Gunnar Nordström [39]; for this reason, the solution has taken the name Reissner-Nordström metric, and it can be written as

$$
d s^{2}=-F_{R N}(r) d t^{2}+F_{R N}(r)^{-1} d r^{2}+r^{2} d \theta^{2}+r^{2} \sin ^{2} d \varphi^{2}
$$

with the function $F_{R N}$ defined as

$$
F_{R N}=1-\frac{2 M}{r}+\frac{Q^{2}}{r^{2}}
$$

with $M$ being the black hole mass and $Q$ the electric charge. Together with the Reissner-Nordström metric (ए.37), the electromagnetic potential which satisfies the equations of motion ( 5.60 ) has the form

$$
A_{\mu}=\left\{-\frac{Q}{r}, 0,0,0\right\}
$$

The Reissner-Nordström solution describes a static, spherically symmetric, asymptotically flat, charged spacetime with an event horizon located at

$$
g^{r r}=0: \quad r_{R N}=M+\sqrt{M^{2}-Q^{2}}
$$


and an inner horizon located at

$$
r_{I H}=M-\sqrt{M^{2}-Q^{2}} .
$$

Hence, the Reissner-Nordström solution describes a static, spherically symmetric, charged black hole, and it can be therefore considered the charged generalisation of the Schwarzschild one, which is recovered in the limit $Q \rightarrow 0$. Interestingly, the Reissner-Nordström solution ([.37) admits a magnetic counterpart, with the electric charge $Q$ replaced by the magnetic charge $P$ and with the electromagnetic potential

$$
A_{\mu}=\{0,0,0,-P \cos \theta\}
$$

In the last century, the Reissner-Nordström spacetime has been studied in depth, and many interestingly features have been discovered: for more details and aspects of the Reissner-Nordström solution, the reader can refer to [26]

\subsubsection{Rotating case: Kerr and Kerr-Newman solution}

We now proceed with a further generalisation of the Schwarzschild solution, considering a non-static spacetime. In fact, from an astrophysical point of view, the assumption of the geometry being static is quite unphysical and simplistic, due to the large amount of angular momentum carried by celestial bodies. Indeed, if we consider a black hole originated from stellar collapse or even more from $\mathrm{BH}-\mathrm{BH}$ merger, it is reasonable to think that the final black hole still carries at least a fraction of the prior total angular momentum, rather than having it completely dissipated or ejected. For this reason, it is natural to wonder whether black hole solutions carrying angular momentum actually exist. Therefore, in order to generalise the Schwarzschild solution while keeping the equations of motion fully analytically solvable, we give up the static assumption in favor of the stationary one. Namely, we require the spacetime (and hence the metric) to be explicitly time independent (i.e. it does not change in time), but it can rotate. Considering for simplicity a vacuum spacetime, we again assume asymptotically flatness and, moreover, we also assume the system to be axisymmetric, with the rotational axis 


\section{INTRODUCTION AND THEORY REVIEW}

being the symmetry axis. With these assumptions and simplifications, the Einstein's equations are now analytically solvable, and they lead to the background metric

$$
\begin{aligned}
d s^{2}= & -\left(1-\frac{2 M r}{\rho^{2}}\right) d t^{2}-\frac{4 M r a \sin ^{2} \theta}{\rho^{2}} d t d \varphi+\frac{\rho^{2}}{\Delta} d r^{2} \\
& +\rho^{2} d \theta^{2}+\left(r^{2}+a^{2}+\frac{2 M r a^{2} \sin ^{2} \theta}{\rho^{2}}\right) \sin ^{2} \theta d \varphi^{2}
\end{aligned}
$$

with

$$
\Delta=r^{2}+a^{2}-2 M r, \quad \rho^{2}=r^{2}+a^{2} \cos ^{2} \theta,
$$

and where $M$ is the mass of the gravitating object and $a=\frac{J}{M}$ its rotation parameter, being $J$ the total angular momentum.

The metric's solution (‥43) is known as Kerr metric, from the mathematician Roy Kerr who first derived its analytical expression in the 1963 [40]. To be precise, the solution (‥43) is the Kerr metric written in the Boyer-Lindquist coordinates frame, since the original solution found by Kerr in 1963 was written in a Eddington-Finkelstein coordinates frame $(v, r, \theta, \varphi)$. The Kerr metric describes an asymptotically flat, axisymmetric, rotating black hole with mass $M$ and rotation parameter $a$, and in the limit $a \rightarrow 0$ the Kerr solution reduces to the Schwarzschild one. As a rotating generalisation of the Schwarzschild metric, the Kerr solution can be derived from the Schwarzschild one using the Newman-Penrose formalism: more details can be found in [21].

Being a black hole, the Kerr spacetime possesses an event horizon; remarkably, that is not the only interesting "exotic" surface the Kerr metric has! As it happens for the Schwarzschild solution, from the metric (‥43) we observe that the surfaces such that

$$
g^{r r}=0
$$

appears to be singularities. However, as in the static case, those are not real spacetime singularities, but rather coordinate singularities, which could be removed by opportune coordinates redefinitions. In fact, the only real spacetime time singularity is located at the point $r=0$, which at first sight can be described by a ring (remember, the spacetime is not spherically symmetric, and the point $r=0$ corresponds to the set of points such that $x^{2}+y^{2}=a^{2}$ in the cartesian plane, 
which describes a circle of radius $a$ ). Anyway, solving $g^{r r}=0$ one finds the two hypersurfaces:

$$
r_{+}=M+\sqrt{M^{2}-a^{2}}, \quad r_{-}=M-\sqrt{M^{2}-a^{2}} .
$$

If $a \neq 0$, they describe ellipsoids in the cartesian space (again, remember that the spacetime is not spherical symmetric, thus hypersurface described by constant radial coordinate are not spheres). The surface with radius $r_{+}$is the black hole's event horizon, separating causally disconnected regions of the spacetime. Instead, the surface with radius $r_{-}$is known as Cauchy horizon, and it is always located inside the event horizon. From (‥46), in order to have "real" horizons we notice that the following condition is required:

$$
0 \leq a \leq M
$$

If $a=0$, the Cauchy horizon disappears and the event horizon is simply $r=$ $2 M=r_{S}$, as the Kerr solution reduces to the Schwarzschild one. If the rotation parameter assumes its largest value $a=M$, the black hole is said to be extremal: in this case, the event and the Cauchy horizons coincide at $r=M$. Remarkably, in the Kerr spacetime there are also other significant surfaces: in fact, by looking at (‥43), we notice that the metric appears to not be regular also at points where $g_{t t}=0$. This should not look too odd, since it also happens in the Schwarzschild/ Reissner-Nordström case; however, in the static, spherically symmetric cases it is $g_{t t}=g^{r r}$, hence this condition is nothing more than the event horizon condition.

On the contrary, in the rotating case $g_{t t} \neq g^{r r}$ and the conditions $g_{t t}=0, g^{r r}=0$ lead to different physical surfaces.

From $g_{t t}=0$ we get

$$
r_{E}=M+\sqrt{M^{2}-a^{2} \cos ^{2} \theta}, \quad r_{I}=M-\sqrt{M^{2}-a^{2} \cos ^{2} \theta},
$$

which are usually referred to as the ergosurface and the inner ergosurface, respectively. Interestingly, we notice that the ergosurface is located outside the event horizon, while it does not happen for the inner one.

Inside the ergosuface (in the region called ergosphere), since $g_{t t}<0$ the world 
lines are space-like; although these considerations are coordinate dependent, they describe a physical, coordinate independent phenomenon known as frame-dragging (or Lense-Thirring effect). In fact, around a rotating black hole the spacetime is forced to rotate with the black hole itself, being dragged by the black hole's rotation; thus, in the ergoregion it is not possible for any object to stay stationary with respect to a distant observer, since it would imply the object being moving faster than light [10, [20]. As we will see later on, the existence of an ergoregion around rotating black holes is crucial for energy extraction processes like the Penrose process [41].

For completeness, we also write the asymptotically flat, stationary, axisymmetric, charged black hole solution of the Einstein-Maxwell action (․30). Such solution, known as Kerr-Newman metric (from Roy Kerr and Ezra Newman), is the rotating generalisation of the Reissner-Nordström black hole, and it is the charged generalisation of the Kerr metric.

The Kerr-Newman metric reads

$$
\begin{aligned}
d s^{2}= & -\left(1-\frac{2 M r-Q^{2}}{\rho^{2}}\right) d t^{2}-\frac{2\left(2 M r-Q^{2}\right) a \sin ^{2} \theta}{\rho^{2}} d t d \varphi+\frac{\rho^{2}}{\Delta} d r^{2} \\
& +\rho^{2} d \theta^{2}+\left(r^{2}+a^{2}+\frac{2 M r a^{2} \sin ^{2} \theta}{\rho^{2}}\right) \sin ^{2} \theta d \varphi^{2}
\end{aligned}
$$

with

$$
\Delta=r^{2}+a^{2}-2 M r+Q^{2}, \quad \quad \rho^{2}=r^{2}+a^{2} \cos ^{2} \theta
$$

where $M$ is the black hole's mass, $a$ is the rotation parameter and $Q$ is the electric charge. The electromagnetic potential which satisfies the equations of motion has the form

$$
A_{\mu}=\left\{-\frac{Q r}{\rho^{2}}, 0,0, \frac{Q a r \sin ^{2} \theta}{\rho^{2}}\right\}
$$

Interestingly, as it happens for the Reissner-Nordström solution, it is easy to write the Kerr-Newman solution including both electric and magnetic charges; in the metric (‥49) it is sufficient to replace $Q^{2}$ with $Q^{2}+P^{2}$, being $P$ the magnetic 
charge, while the electromagnetic potential becomes

$$
A_{\mu}=\left\{\frac{-Q r+P a \cos \theta}{\rho^{2}}, 0,0, \frac{Q a r \sin ^{2} \theta-P\left(r^{2}+a^{2}\right) \cos \theta}{\rho^{2}}\right\} .
$$

In the following chapters, we will often refer to the electromagnetic ReissnerNordström or Kerr-Newman solution as the "dyonic" solutions, meaning that we consider solutions with both electric and magnetic charges.

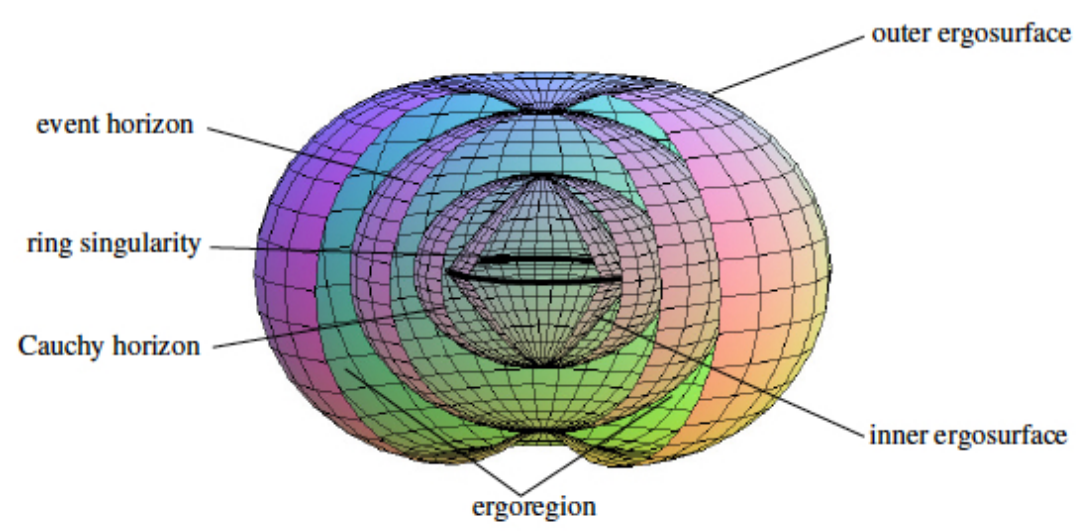

Figure 1.1: Horizons, ergosurfaces and singularity for a Kerr black hole, with $a<M$. Picture from "Schwarzschild and Kerr Solutions of Einsteins Field Equation: an introduction", by C. Heinicke and F. W. Hehl [1]].

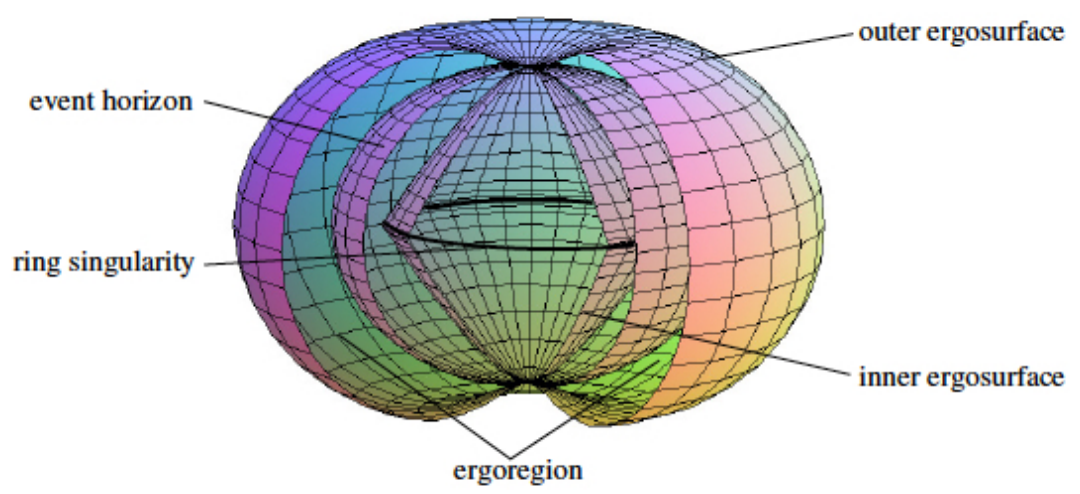

Figure 1.2: Horizons, ergosurfaces and singularity for an extremal Kerr black hole, with $a=M$. Picture from "Schwarzschild and Kerr Solutions of Einsteins Field Equation: an introduction", by C. Heinicke and F. W. Hehl [1]]. 


\subsubsection{Black hole thermodynamics}

Starting from the Sixties, thanks to the discoveries by Kerr and Newman the black hole physics regained much popularity among the scientific community. Many authors, both mathematicians and physicists, focused their researches on a deeper comprehension of black holes nature and their properties; in particular, authors like Smarr, Virbhadra, Christodoulou, Ruffini, Bekenstein, Hawking, Penrose and others, made game changing discoveries on what concerns the black holes structure and energy. Remarkably, they have been the pioneers of the black hole thermodynamics era, which is still a fascinating and intensively studied topic in modern theoretical physics [42-44].

For the purpose of this work, this section will contain only the basic concepts concerning the black hole energy and thermodynamics, since they will turn out to be useful in the next chapters. For more detailed reviews or notes, the reader can look at [2I, 44-46].

Considering as the most generic case a Kerr-Newman black hole, the energy contained in a sphere of radius $r$ such that $r$ is larger than some finite $R>r_{+}$is given by [47]

$$
E=M-\frac{Q^{2}}{R}\left(\frac{a^{2}}{3 R^{2}}+\frac{1}{2}\right)
$$

The result above shows how the energy of an uncharged $(Q=0)$ black hole is entirely contained within the event horizon and it is precisely its mass; on the contrary, for charged black holes there is a contribution to the total energy coming from the exterior region, and such contribution is larger for rotating black holes $(a \neq 0)$. In the case of rotating black holes, through the existence of an ergosphere Penrose [4]] showed how it is possible to extract rotational energy from a black hole; thus, one could ask if it is possible to totally extract rotational energy from a spinning black hole, causing it to stop the rotation. On this topic, Christodoulou and Ruffini [48] showed that there is a threshold amount of energy which cannot be extracted in any way; this quantity, usually named irreducible mass, is given by

$$
M_{i r r}^{2}=\frac{r_{+}^{2}+a^{2}}{4}
$$


and it cannot be decreased by any process, reversible or irreversible:

$$
d M_{i r r} \geq 0
$$

The condition above turns out to be the first step towards the black hole thermodynamics. In fact, thanks to the intuitions of Bekenstein [4.9], Penrose [41] and Hawking [50], an easy relationship between the black hole's entropy $S_{B H}$ and its horizon's area $A_{H}$ has been found:

$$
S_{B H} \propto A_{H},
$$

where the horizon's area is given by

$$
A_{H}=r_{+}^{2}+a^{2}
$$

Using the result ([.54), we can write

$$
d S_{B H}=8 \alpha M_{i r r} d M_{i r r} \geq 0
$$

which is exactly the second law of thermodynamics ${ }^{m}$.

In general, it is possible to write the four laws of black hole thermodynamics [45] in the following way:

- Zeroth Law: the black hole's $T_{B H}$ temperature is constant over the event horizon.

- First Law: the total energy is always conserved.

- Second Law: the entropy of an isolated black hole never decreases, $d S_{B H} \geq 0$.

- Third Law: $T_{B H}$ can never be reduced to zero in a finite number of steps.

\footnotetext{
${ }^{1}$ The proportionality constant $\alpha$ has been computed by Hawking to be $\alpha=\frac{c^{6}}{4 G \hbar}$
} 
In particular, the first law can be compactly written with the following equation, known as Smarr formula [17]:

$$
\delta M=\frac{\kappa}{2} \delta A_{H}+\Omega_{H} \delta J_{H}+\Phi_{B H} \delta Q
$$

where $\kappa$ is the horizon's surface gravity, $\Omega_{H}$ is the horizon's angular velocity and $\Phi_{B H}$ is the electric potential on the horizon.

\subsection{Modified Gravity: introduction}

In this section we briefly discuss testing General Relativity and other modified gravity theories. Since a detailed and exhaustive discussion about such a huge research area is far beyond the possibilities and the aims of this work, we will introduce and illustrate the main aspects of this topic to provide the reader a basic knowledge about the motivations and the opportunities of modifying Einstein's theory of gravity. In particular, we will focus our attention on aspects regarding the possibility of testing General Relativity and other theories of gravity through black hole physics and related phenomena.

So, this section is structured as follows: in the first part we will motivate the need to test General Relativity and alternative gravity theories; in the second section, we will provide a picture of the many possibilities to modify General Relativity, with particular interest in theories involving scalar fields; finally, in the last section we will shortly discuss testing gravity theories with strong gravity regime observations (such as gravitational waves), and the impact of the recent GWs detections on the plethora of modified gravity theories. However, since this section cannot be an exhaustive review on modified gravity and related observations, we have decided to focus our attention on those aspects which will be crucial for the results discussed in the next chapters. For exhaustive reviews, the reader can refer to [2, 8, 5]].

\subsubsection{Beyond General Relativity}

Since its publication in 1915, it was immediately clear that the theory of General Relativity would have completely changed the approach to gravity and to exper- 
iments. Moreover, some of General Relativity's predictions were tested and confirmed with extraordinary precision only a few years after 1915 (in 1919 the light bending was tested, and in 1925 a group of scientists claimed to have measured the gravitational redshift), giving great credibility to GR and fueling the enthusiasm for this revolutionary theory. In addition, during the last century many tests of GR have been made, again providing results in incredible accordance with the theory predictions (Shapiro delay, binary pulsars timing, etc.). However, despite of the elegance and the outstanding precision of its predictions, since its publication GR had some compatibility issues with cosmology, leading Einstein himself to add and then remove the cosmological constant in his equations trying to motivate the stationarity/dynamicity of the Universe:

$$
R_{\mu \nu}-\frac{1}{2} R g_{\mu \nu}+\Lambda g_{\mu \nu}=2 T_{\mu \nu}
$$

with $T_{\mu \nu}$ being the stress-energy tensor and $\Lambda$ being the cosmological constant. Initially, Einstein added the constant term to the equation to prevent the prediction of a collapsing or expanding Universe; however, after the astronomer Edwin Hubble discovered in 1929 that the Universe is actually expanding, Einstein removed the constant term from the field equations. Nowadays, we have evidence that the Universe is accelerating its expansion rate, and thus we need a source fueling such acceleration in contrast with the effect of gravity, which on the contrary tends to slow down the expansion. Such mysterious and still unobserved source of energy has been named ${ }^{\varpi}$ dark energy, and current observations indicate that is roughly $72 \%$ of the content of the Universe [52]. Although unknown, a simple way to model the effects of dark energy is through the introduction of a constant term in the Einstein's equation, acting like a repulsive force which causes the Universe to accelerate its expansion (paradoxically, Einstein added the cosmological constant to keep the Universe stationary, while we need it to explain the Universe expansion!). However, to be consistent with the current observations the value of the cosmological constant must be incredibly small (in Planck units): this is a deep fine tuning problem, and currently it cannot be motivated by any theory prediction (the vacuum energy predicted by quantum field theory is 120 orders of

\footnotetext{
${ }^{1}$ By the cosmologist Michael Turner in 1998.
} 
magnitude bigger than the needed value of the cosmological constant, making it " The worst theoretical prediction in the history of physics!" [53]]).

Besides dark energy, the remaining part of the content of the Universe is composed by "ordinary matter" (elementary particles and the complex particles they form) for the $4.6 \%$ and by dark matter for the remaining $23 \%$ (see Fig.‥3 below).

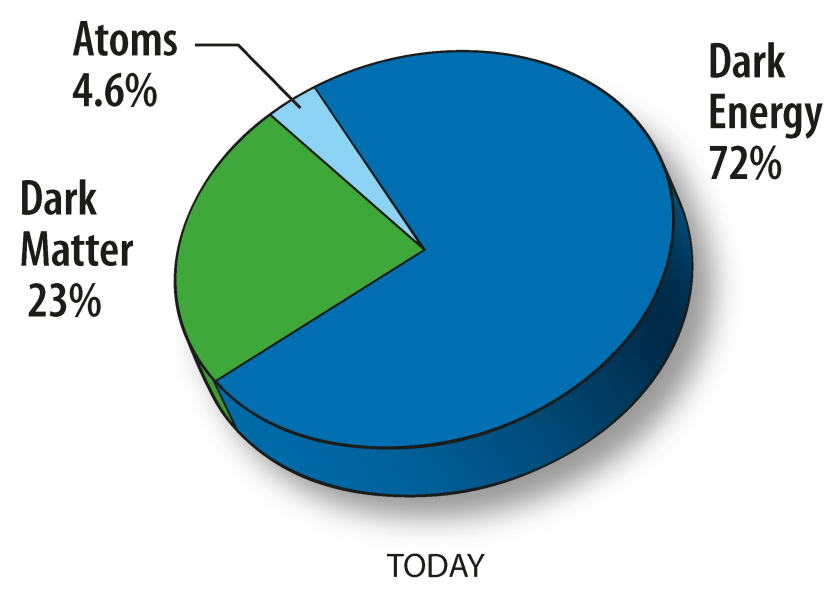

Figure 1.3: Picture "Content of the Universe Pie Chart", from Wilkinson Microwave Anisotropy Probe (WMAP), National Aeronautics and Space Administration. Since the WMAP data is accurate to two digits, the total of these numbers is not $100 \%$.

Dark matter roughly accounts for $82 \%$ of the total matter in the Universe. It is called "dark" because it does not interact with light, and thus it is almost undetectable with the majority of current astronomical telescopes and detectors. Therefore, we can infer the existence of this form of matter by indirect observations, such as the galaxies rotation curves or in the Cosmic Microwave Background (CMB): for a modern theory of dark matter and its possible effects, the reader can refer to [i]].

At the moment, the main cosmological model including both dark energy and dark matter is the so called $\Lambda$ CDM model, where $\Lambda$ refers to the cosmological constant associated with dark energy and "CDM" stands for Cold-Dark-Matter, 
meaning that its speed is small compared to the speed of light. However, since the presence of such a small cosmological constant term in the Einstein's equation cannot be motivated by any quantum field theory, in the last decades many attempts to modify the Einstein's theory of gravity without the introduction of the cosmological constant have been proposed.

Moreover, the lack of a renormalizable quantum theory of gravity has motivated the attempts to formulate theories of gravity which could be consistent with quantum field theory, providing predictions on how GR should be modified at high energies. Such theories, commonly referred to as modified gravity theories, must be considered extensions of the General Relativity theory, in the sense that they must agree with observations and GR predictions at well tested energy scales (the weak-field regime, $10^{-6} \mathrm{~m} \leq l \leq 10^{11} \mathrm{~m}$ ), while being able to explain high and low energy (cosmological) phenomena which cannot be explained with GR. In other words, modified gravity theories are attempts to modify General Relativity at both low and high energy scales, while being consistent with GR in the weakfield gravity regime, where GR has been largely and successfully tested. Being black holes and other compact objects, such as neutron stars and pulsars, the best natural laboratories to test strong-field gravity, it is therefore important to study such objects in alternative theories of gravity, since theories which differ from GR generically predict different dynamics (such as gravitational waves formation and propagation).

\subsubsection{Modified gravity theories: a roadmap}

In the past years, there has been a plethora of attempts to modify GR to find alternative theories of gravity (see [ $]]$ for a review). In order to determine whether a theory of gravity is valid or not, it must satisfy some requirements; however, there is nothing like a validity paradigm, and the final decision about the reliability of a given theory is reasonably arbitrary.

From our point of view, some reasonable criteria to check the validity of an alternative theory of gravity are:

- compatibility with intermediate energy tests, thus being consistent with GR at those scales; 


\section{INTRODUCTION AND THEORY REVIEW}

- compatibility with cosmological evidence;

- compatibility with the existence of compact objects, thus describing their structure and predicting their formation;

- compatibility with the detected gravitational wave signals;

- derivation from an action principle.

In particular, in order to satisfy the intermediate energies compatibility requirement, many modified gravity theories introduce some screening effects to hide some extra degrees of freedom which are predicted at higher/lower energy scales but they do not appear at intermediate scales. Among these screening mechanisms, we mention the Vainshtein mechanism [54, 55], chameleons [56, 57] and MOND dynamics [58-62].

Due to the lack ${ }^{\square}$ of stringent tests and constraints, in the last 40-50 years the number of proposed alternative theories of gravity has been very rapidly increasing. To move in such a vast space and to avoid being lost, we will use the Lovelock's theorem [63, 64] as guiding principle. As written in [2], the Lovelock's theorem states that in four spacetime dimensions the only divergence-free symmetric rank-2 tensor constructed solely from the metric and its derivatives up to second differential order, and preserving diffeomorphism invariance, is the Einstein tensor plus a cosmological term:

$$
R_{\mu \nu}-\frac{1}{2} R g_{\mu \nu}+\Lambda g_{\mu \nu}=2 T_{\mu \nu}
$$

Moreover, if we assume that the Einstein's equation (‥6]) comes from an action principle, we single out the Einstein-Hilbert action

$$
S=\int d^{4} x \sqrt{-g} \frac{R}{4}+S_{M}\left[\psi_{M}, g_{\mu \nu}\right]
$$

where $S_{M}$ denotes the matter sector with $\psi_{M}$ denoting the matter fields.

Thus, according to the Lovelock's theorem, there is not much space left to modify

\footnotetext{
${ }^{1}$ We will discuss in more details about the constraints coming from gravitational waves detection and in particular GW170817 in the following subsections.
} 
Einstein theory of gravity. However, like all theorems do, Lovelock's theorem holds since its assumptions do; thus, relaxing any of its assumptions, it is possible to circumvent the theorem and to end up with a different and a priori viable theory of gravity. The main four possibilities to circumvent the Lovelock's theorem and hence generating gravity theories alternative to GR are:

- Additional fields: since the Lovelock's theorem assumes the metric and its connection being the only dynamical field on the LHS of the Einstein's equation, the easiest and most common way to circumvent the theorem is by adding extra degrees of freedom, namely extra fields (scalar, vector or tensor) coupled to the metric tensor; however, introducing additional degrees of freedom non minimally coupled to gravity usually leads to instabilities (see Ostrogadski's theorem [6.5] for more details). Some examples of gravity theories obtained by introducing additional fields are scalar-tensor theories (including Galileons), $f(R)$ gravity, Quadratic gravity, Einstein-Aether-Horava-Lifshitz, Bimetric gravity, Generalised Proca.

- Diffeomorphism invariance violation: the first way to violate diffeomorphism invariance is by violating Lorentz invariance at high energy scales, hence assuming that such invariance is an emergent symmetry appearing just at low and intermediate energy scales, where it is very well tested. In addition, another possibility to violate diffeomorphism invariance is by assuming the graviton being a slightly massive spin-2 field rather than a massless one, although the mechanism through the graviton can acquire mass is not clear yet.

- Higher dimensions: another possibility to circumvent the Lovelock's theorem is by assuming the existence of more than four spacetime dimensions, as it happens in string theory scenarios. With this assumption, the higher dimensional theory of gravity naturally exhibits additional fields when reduced to four dimensions [ [66,, 67$]$, hence motivating possible deviations from GR at high energy scales.

- Weak Equivalence Principle (WEP) violation: at the end, another option to circumvent the Lovelock's theorem is by dropping the assumption 


\section{INTRODUCTION AND THEORY REVIEW}

of having a divergence-free Einstein tensor and energy-stress tensor, consequently violating the Weak Equivalence Principle.

Since an exhaustive review of so many alternative theories of gravity is beyond the aim of this work, we schematically summarize the different paths to circumvent the Lovelock's theorem and generate alternative theories of gravity in Fig.(ㄸ. $)$. For an up-to-date and detailed review, the reader can refer to [2].

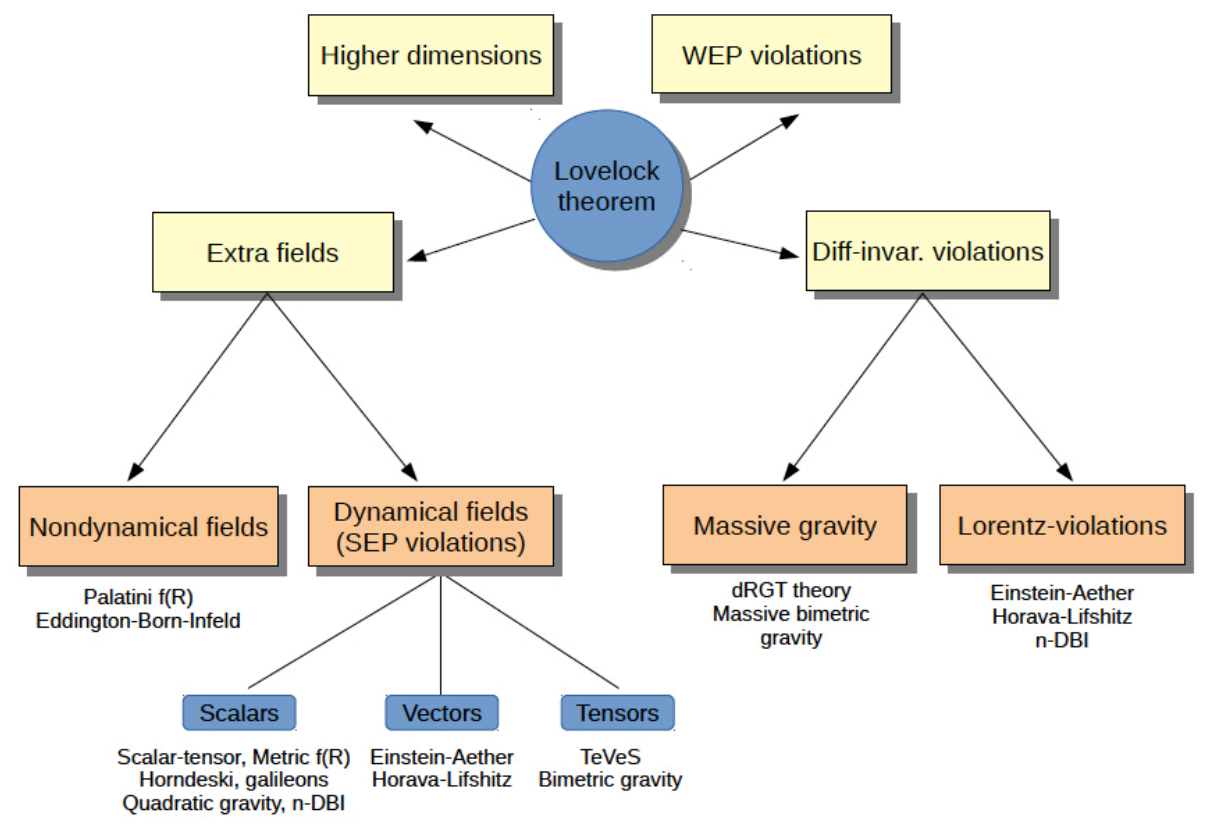

Figure 1.4: The diagram, taken from [圆], illustrates the different ways to circumvent the Lovelock's theorem by relaxing any of its assumptions, ending up with different alternative theories of gravity.

As far as this work is concerned, to motivate the backgrounds we will consider in the next chapters, we now very briefly show the basic features of some modified gravity theories; in particular, we focus our attention on scalar-tensor gravity [68] and quadratic gravity [69]. 


\section{Scalar-tensor gravity}

As we have previously discussed, a possible way to try to extend GR is by adding additional fields non-minimally coupled to the metric tensor. If we consider a single scalar field $\phi$ coupled to the metric, the most general action of scalar-tensor gravity can be written in the following way:

$$
S=\int d^{4} x \sqrt{-g}\left[\phi R-\frac{\omega(\phi)}{\phi} g^{\mu \nu}\left(\partial_{\mu} \phi\right)\left(\partial_{\nu} \phi\right)-V(\phi)\right]+S_{M}\left[\psi_{M}, g_{\mu \nu}\right]
$$

where $\omega$ and $V$ are generic functions of the scalar field and with $S_{M}$ denoting the matter action, which is function both of the metric tensor $g_{\mu \nu}$ and the collective matter fields $\psi_{M}$. In the form ([.6.3), the scalar field is non minimally coupled to the metric tensor (the Ricci scalar is directly coupled to the the scalar field); in this form, the scalar-tensor action is said to be in the Jordan frame.

If we perform a redefinition of the scalar field

$$
\varphi=\varphi(\phi)
$$

and a conformal transformation of the metric

$$
g_{\mu \nu} \rightarrow \tilde{g}_{\mu \nu}=A^{-2} g_{\mu \nu}
$$

with the particular choice

$$
A(\varphi)=\phi^{-1 / 2}
$$

we can recast the action $\left(\left[\frac{6.37)}{)}\right.\right.$ in the so-called Einstein frame, where it takes the form

$$
S=\int d^{4} x \sqrt{-\tilde{g}}\left[\tilde{R}-2 \tilde{g}^{\mu \nu}\left(\partial_{\mu} \varphi\right)\left(\partial_{\nu} \varphi\right)-W(\varphi)\right]+S_{M}\left[\psi_{M}, A^{2}(\varphi) \tilde{g}_{\mu \nu}\right]
$$

where the potential is $W(\varphi)=A^{4}(\varphi) V(\phi(\varphi))$. In the Einstein frame, the scalar field is minimally coupled in the gravitational sector but not in the matter one, with the matter fields being directly coupled to the scalar field. The Jordan and the Einstein frames provide two different representations of the same theory; thus, the meaningful, physical quantities obtained through an experiment do not depend 


\section{INTRODUCTION AND THEORY REVIEW}

on the chosen frame, provided that their physical units are scaled by the appropriate conformal factors. This aspect should not be considered a technicality or a quibble: since in the Einstein frame the scalar field is non minimally coupled to the matter sector, to study a non vacuum system we need to exactly know how the scalar field is coupled to matter, to consequently rescale the measured/computed quantities (for example, a test particle follows the Jordan-frame geodesics, not the Einstein-frame ones). For a more detailed discussion on the equivalence between Jordan/Einstein frames, the reader can refer to [[i], [1]].

In the Jordan frame, the field equations of scalar-tensor gravity are

$$
\begin{aligned}
& G_{\mu \nu}=\frac{2}{\phi} T_{\mu \nu}+\frac{\omega(\phi)}{\phi^{2}}\left(\partial_{\mu} \phi \partial_{\nu} \phi-\frac{1}{2} g_{\mu \nu} \partial_{\gamma} \phi \partial^{\gamma} \phi\right)+\frac{1}{\phi}\left(\nabla_{\mu} \nabla_{\nu} \phi-g_{\mu \nu} \square \phi\right)-\frac{V(\phi)}{2 \phi} g_{\mu \nu}, \\
& \square \phi=\frac{1}{3+2 \omega(\phi)}\left(2 T-4 \phi \frac{\partial T}{\partial \phi}-\frac{d \omega}{d \phi} \partial_{\sigma} \phi \partial^{\sigma} \phi+\phi \frac{d V}{d \phi}-2 V(\phi)\right),
\end{aligned}
$$

with the energy-stress tensor defined as

$$
T^{\mu \nu}=-2(-g)^{-1 / 2} \frac{\delta S_{M}\left(\psi_{M}, g_{\mu \nu}\right)}{\delta g_{\mu \nu}} .
$$

Instead, in the Einstein frame the field equations simply read

$$
\begin{aligned}
& \tilde{G}_{\mu \nu}=2\left(\partial_{\mu} \varphi \partial_{\nu} \varphi-\frac{1}{2} \tilde{g}_{\mu \nu} \partial_{\rho} \varphi \partial^{\rho} \varphi\right)-\frac{1}{2} \tilde{g}_{\mu \nu} V(\varphi)+2 \tilde{T}_{\mu \nu} \\
& \tilde{\square} \varphi=-a(\varphi) \tilde{T}+\frac{1}{4} \frac{d W}{d \varphi},
\end{aligned}
$$

with

$$
a(\varphi)=\frac{d[\ln A(\varphi)]}{d \varphi}
$$

and with the energy-stress tensor defined as

$$
\tilde{T}^{\mu \nu}=-2(-g)^{-1 / 2} \frac{\delta S_{M}\left(\psi, A^{2} \tilde{g}_{\mu \nu}\right)}{\delta \tilde{g}_{\mu \nu}} .
$$

Remarkably, there are some phenomena predicted by scalar-tensor gravity and not occurring in GR which could lead to detectable effects. Among these, we mention 
the emission of dipolar gravitational radiation from compact binaries (rather than the quadrupolar radiation predicted by GR) [ [r2], the spontaneous scalarization phenomenon [[r.3] for extreme compact objects and the superradiance effect for black holes. For what concerns this work, we are particularly interested in the superradiance phenomenon, and therefore we will dedicate to it a detailed discussion in the next sections.

In four spacetime dimensions, the fundamental scalar-tensor gravity theory leading to second-order field equations is Horndeski gravity [74, [75], whose action can be written in the form:

$$
\begin{aligned}
S= & \int d^{4} x \sqrt{-g}\left\{K(\phi, X)-G_{3}(\phi, X) \square \phi\right. \\
& +G_{4}(\phi, X) R+G_{4, X}(\phi, X)\left[(\square \phi)^{2}-\left(\nabla_{\nu} \nabla_{\nu} \phi\right)\left(\nabla^{\mu} \nabla^{\nu} \phi\right)\right] \\
& +G_{5}(\phi, X) G_{\mu \nu} \nabla^{\mu} \nabla^{\nu} \phi-\frac{G_{5, X}(\phi, X)}{6}\left[(\square \phi)^{3}-3 \square \phi\left(\nabla_{\mu} \nabla_{\nu} \phi\right)\left(\nabla^{\mu} \nabla^{\nu} \phi\right)\right. \\
& \left.\left.+2\left(\nabla_{\mu} \nabla_{\nu} \phi\right)\left(\nabla^{\mu} \nabla_{\rho} \phi\right)\left(\nabla^{\nu} \nabla^{\rho} \phi\right)\right]\right\} .
\end{aligned}
$$

with $X=\frac{1}{2} \partial^{\mu} \phi \partial_{\mu} \phi$ being the kinetic term of the scalar field and $G_{i, X}$ denoting the derivative of the $G_{i}$ functions with respect to $X$.

Thus, depending on the particular choices for the functions $G_{i, X}$, it is possible to recover many other scalar-tensor theories, such as Brans-Dicke [[6], Chameleons [56, 57] and, obviously, General Relativity. Remarkably, it has been recently shown that it is possible to further generalise the Horndeski action to a larger class of gravity theories (known as DHOST [[7]) without propagating ghost modes.

For completeness, we conclude by writing the general scalar-tensor theory action when gravity is coupled to more than one scalar field:

$$
S=\int d^{4} x \sqrt{-g}\left[F(\phi) R-\gamma_{a b}(\phi) g^{\mu \nu} \partial_{\mu} \phi^{a} \partial_{\nu} \phi^{b}-V(\phi)\right]+S_{M}\left[\psi_{M}, g_{\mu \nu}\right]
$$

with $\gamma_{a b}$ being the metric of the target space defined by the scalar fields. 


\section{INTRODUCTION AND THEORY REVIEW}

\section{Quadratic gravity}

From a quantum field theory point of view, Einstein's theory of gravity is not renormalizable, and therefore it cannot be considered a viable option for a quantum theory of gravity. However, things change if we consider the Einstein-Hilbert term being the first term in an expansion containing all possible curvature invariants: at small energy scales, the theory can be truncated and the EinsteinHilbert action is a valid "representation" of the theory (decoupling limit), while at higher-energy scales the full general theory is required. Remarkably, it has been shown that the gravity theory becomes renormalizable when quadratic curvature terms are included in the action [[r] , without the need of higher curvature terms contributions. However, including quadratic curvature terms in the action yields to higher-derivative terms appearing in the field equations, which usually lead to ghosts and Ostrogradski's instabilities.

At second order in the curvature, there exist only few independent algebraic curvature invariants:

$$
\begin{gathered}
R^{2}, \quad R_{\mu \nu}^{2}=R^{\mu \nu} R_{\mu \nu}, \quad R_{\mu \nu \sigma \rho}^{2}=R_{\mu \nu \sigma \rho} R^{\mu \nu \sigma \rho} \\
\tilde{R}^{\mu \nu \sigma \rho} R_{\mu \nu \sigma \rho}=\frac{1}{2} \epsilon^{\mu \nu \alpha \beta} R_{\mu \nu \sigma \rho} R_{\alpha \beta}^{\rho \sigma}
\end{gathered}
$$

with $\epsilon^{\mu \nu \alpha \beta}$ being the Levi-Civita tensor and $\tilde{R}^{\mu \nu \sigma \rho} R_{\mu \nu \sigma \rho}$ the Chern-Simons (or Pontryagin) scalar (we will discuss in more details about the Levi-Civita tensor and the Chern-Simons scalar in chapter 母) .

Another important curvature scalar, known as Gauss-Bonnet scalar, can be constructed as a combination of the curvature invariants in the following way:

$$
R_{G B}=R^{2}-4 R_{\mu \nu}^{2}+R_{\mu \nu \sigma \rho}^{2}
$$

In particular, both the Gauss-Bonnet scalar and the Chern-Simons one have been largely studied in gravity theories, since they naturally emerge in low-energy reductions of string theory, being respectively coupled with the dilaton and the axion fields $[\mathbf{[ 7 9}, \mathbf{8 1}]$. Since both terms are topological-invariants in four spacetime dimensions, they do not lead to modifications of the field equations; however, when coupled to extra dynamical fields (such as scalar ones), their contributions to field 
equations become relevant, leading to deviations from Einstein's gravity.

Including a single scalar field coupled to all the possible quadratic curvature invariants, the most generic action can be written as

$$
\begin{aligned}
S=\frac{1}{4} & \int d^{4} x \sqrt{-g}\left[R-2 \nabla_{\mu} \phi \nabla^{\mu} \phi-V(\phi)+f_{1}(\phi) R^{2}+f_{2}(\phi) R_{\mu \nu} R^{\mu \nu}\right. \\
& \left.+f_{3}(\phi) R_{\mu \nu \sigma \rho} R^{\mu \nu \sigma \rho}+f_{4}(\phi) \tilde{R}^{\mu \nu \sigma \rho} R_{\mu \nu \sigma \rho}\right]+S_{M}\left[\psi_{M}, \gamma(\phi) g_{\mu \nu}\right]
\end{aligned}
$$

with $V(\phi)$ being the scalar self-potential and $f_{i}(\phi)$ being the coupling functions. Due to the presence of the quadratic curvature terms, the theory (‥77) is generically unstable, namely it manifests ghosts and instabilities; however, if the curvature invariants enter in the action precisely in the Gauss-Bonnet combination ([.76]), the field equations are free of such ghosts and instabilities.

Apart from Gauss-Bonnet gravity theory [ [ㅍ] ], another way to prevent instabilities is to consider the action (ए.T7) as an effective action of an effective field theory (EFT), namely a truncation of a more general theory (for an introductory review to EFTs, the reader can look at [82]). Using the EFT approach, we can study the particular case of Chern-Simons gravity theory [15, ए6] by setting $f_{1}=f_{2}=f_{3}=0$ in the action (एँ]). The Chern-Simons gravity action reads:

$$
S=\frac{1}{4} \int \sqrt{-g} d^{4} x\left[R-2 \nabla_{\mu} \phi \nabla^{\mu} \phi-V(\phi)+g_{C S} \phi \tilde{R}^{\mu \nu \sigma \rho} R_{\mu \nu \sigma \rho}\right]
$$

with $g_{C S}$ being the coupling constant. To be precise, the action (ㅍ.78) describes the so-called dynamical Chern-Simons gravity (dCS), being $\phi$ a dynamical field with its own kinetic term and eventually with a potential $V(\phi)$. In fact, a different version of Chern-Simons gravity action also exists: such theory, called non dynamical Chern-Simons gravity (ndCS), does not consider the scalar field $\phi$ being dynamical, and therefore no kinetic term appears in the action. Although they could appear similar, the two theories are actually very different: for what concerns this work, we are much more interested in the dynamical theory, since it predicts non standard black hole solutions and parity-breaking deviations from GR [16, 83]. 


\section{INTRODUCTION AND THEORY REVIEW}

\subsubsection{Tests of gravity theories}

To conclude this brief overview of modified gravity, we spend some few words on possible effects and observations which could constrain the theories we have discussed above. First of all, it should be clear at this point that eventual deviations from GR should be especially investigated at strong gravity regime. Therefore, since we are still very far from being able to recreate in laboratory strong gravity environments, we need observations of phenomena occurring at those energy scales. As we have already mentioned, Solar System scale provides extraordinary accurate tests for GR, and thus we have to push the gravity regime even higher if we want to observe any deviation from Einstein's theory. Luckily, Einstein's theory provides the perfect candidates to observe gravity in a strong regime: black holes. For one century they have been elegant and fascinating speculations, associated with phenomena like gravitational lensing and AGNs, but with GW150914 and later on with the Event Horizon Telescope "picture" they have finally become detectable objects [3-[36]. So, at the time we are writing, they are by far the best laboratories we have access to for testing GR and other modified gravity theories. First, their very existence can be considered a preliminary test: in fact, given a generic theory of gravity, it is not trivial to predict the existence of extreme compact objects like black holes, and therefore their predictability can be considered a first requirement for alternative theories of gravity. Furthermore, black holes predicted by General Relativity are remarkably simple since they are described by only few parameters, while in alternative theories of gravity such simplicity is not granted (more details in the next sections). For these reasons, in the last decades much effort has been dedicated to the study of black holes in modified gravity theories, looking for similarities or distinctive differences between GR and other black hole solutions.

However, the game changer in both gravity and astronomy/astrophysics scientific community was the detection in 2015 of the gravitational wave event GW150914 [3, 5], generated by a binary black holes system merging. After GW150914, other gravitational waves events have been detected (at the time we are writing, a dozen of GWs detection have been confirmed, including GW170817 coming from a binary neutron stars system [37]): in all cases, the GR predictions (dipole radiation, 
waveform, etc..) show great agreement with the observations [84, 85]. Thus, any viable alternative theory of gravity must predict the formation and propagation of gravitational waves, and their physical properties (amplitude, energy carried, speed, etc..) must be in accordance with observations. In particular, the detection GW170817 had great impact on setting constraints on the space parameters of gravity theories. In fact, coming from a binary neutron stars system, the GW signal was much longer than the previous BH-BH ones; moreover, in GW170817 the GW signal was followed by an electromagnetic one (a short gamma ray burst), with roughly 1.74 seconds delay [86]. Such electromagnetic counterpart sets very stringent constraints on the speed of propagation of the GWs, with deviations from the speed of light smaller than one part on $10^{15}$. We stress the importance of this result because it finally shed light on the GWs propagation velocity, which previously to GW170817 had not stringent constraints and it was therefore very debated and uncertain, with very different predictions from different theories of gravity: with GW170817, many modified gravity theories predicting a speed of propagation of GWs different from the speed of light have been strongly constrained or even almost completely ruled out (see [87-90] for technical analysis of the constraints on the GWs propagation velocity in alternative gravity theories set by GW170817). Moreover, GW170817 also provided tests to the GWs dispersion relation, the number of spacetime dimensions and the waves polarization: see [85] for details.

Since this section is meant to provide the basic background needed for a contextualization of the results discussed in the next chapters, as far as this work is concerned we conclude this brief overview on the main aspects of modified gravity.

\subsection{Axion cosmology: the basics}

In this section we introduce the basic ideas of axion cosmology, providing a schematic, minimal review of the basic concepts of this large topic. In particular, since for the purposes of this work we are interested in motivating the existence of axions and other light scalar fields and their implications for cosmology and black hole 


\section{INTRODUCTION AND THEORY REVIEW}

physics, in this short review we will focus our attention on the different possibilities to motivate the existence of such boson fields. As we will see, axions and light scalar fields can provide good candidates for solving important cosmological open questions, such as the cosmological constant, dark energy, dark matter and even inflation. For a detailed and exhaustive review on axion cosmology, the reader can refer to [9]].

Nowadays, the word axion can refer to a large class of pseudoscalar fields (namely scalar fields changing sign under parity transformation), appearing in several different contexts and with different origins and motivations. Originally, the term was used in the late Seventies [92] referring to the light scalar particle solving the strong-CP problem according to the Peccei-Quinn mechanism [93, 94] and, in this context, the term indicates how such scalar field is associated with the axial QCD anomaly. However, in the following years the word axion has been applied to many different scalar fields: in quantum field theory, the term can refer to pseudoscalar Goldstone bosons arising from spontaneously broken global symmetries [95]; in string theory, the term is used referring to scalar fields arising from compactified dimensions [96, 97]; in astrophysics and cosmology, the term refers to generic light pseudoscalar fields.

Let's start from its original meaning.

The strong-CP problem in $\mathrm{QCD}^{\square}[14,98]$ is a challenging and intricate open question, which has seen many different attempts of solution in the last 50 years.

With our convention $c=\hbar=G=4 \pi=1$, the QCD lagrangian (including the axion field $a$ and the topological $\theta$-vacua term [95]) reads

$$
\mathcal{L}_{Q C D}=-\frac{1}{4} G_{\mu \nu}^{b} G^{b, \mu \nu}+\sum_{q} \bar{q}\left(i \gamma^{\mu} D_{\mu}-m_{q}\right) q+\frac{g_{s}^{2}}{8} \theta G_{\mu \nu}^{b} \tilde{G}^{b, \mu \nu}
$$

with $G_{\mu \nu}$ being the gluon field strength, $D_{\mu}$ the gauge covariant derivative, $q$ being the quarks with their respective mass $m_{q}$ and $g_{s}$ being the strong coupling constant.

\footnotetext{
${ }^{1}$ The strong-CP problem is based on the fact that, according to the experiments, QCD preserves the CP-symmetry, although such symmetry is not protected and a CP violating terms naturally appear in the action.
} 
The CP-violating term

$$
\mathcal{L}_{Q C D}^{\theta}=g_{s}^{2} \frac{\theta}{8} G_{\mu \nu} \tilde{G}^{\mu \nu}
$$

induces an electric dipole moment for the neutron given by [9.9]:

$$
d_{N}=3.6 \cdot 10^{-18} \theta \text { e m }
$$

However, the observed, constrained electric dipole moment is [1010]:

$$
\left|d_{N}\right|<2.9 \cdot 10^{-28} \mathrm{em}
$$

which implies a value for the $\theta$ angle close to zero:

$$
\theta \lesssim 10^{-10}
$$

This is a fine tuning problem, since there is apparently no explicit reason to motivate an approximately zero $\mathrm{CP}$ violating term. A mechanism to motivate such small value for the $\theta$ angle was proposed by Peccei and Quinn [93] : the idea is to promote $\theta$ to a field and to introduce a new global, chiral $U(1)_{P Q}$ symmetry, spontaneously broken at some scale $f_{a}$ and with an associated pseudo-NambuGoldstone boson ${ }^{\mathbb{m}}$, known as the axion. This axion, that we will denote with " $a$ ", enjoys a shift symmetry

$$
a=a+\text { const },
$$

and it couples with the $G \tilde{G}$ in the QCD lagrangian, which reads

$$
\mathcal{L}_{Q C D+a}=-\frac{1}{4} G_{\mu \nu}^{b} G^{b, \mu \nu}+\frac{1}{2} \partial_{\mu} a \partial^{\mu} a+\sum_{q} \bar{q}\left(i \gamma^{\mu} D_{\mu}-m_{q}\right) q+\frac{g_{s}^{2}}{8}\left(\theta+\frac{\mathcal{C} a}{f_{a}}\right) G_{\mu \nu}^{b} \tilde{G}^{b, \mu \nu}
$$

with $\mathcal{C}$ being the colour anomaly [9.5].

As showed in [93], due to the periodicity of the expectation value $<G \tilde{G}>$ the axion is forced to take the vev

$$
<a>=-\frac{f_{a}}{\varrho} \theta
$$

\footnotetext{
${ }^{1}$ Due to instantons effects, the axion acquires a small mass, thus ceasing to be an authentic massless Nambu-Goldstone boson and therefore gaining the appellative "pseudo".
} 
which therefore cancels out the $\mathrm{CP}$ violating term in the lagrangian (‥85) and solves the CP problem.

Due to QCD instantons effects [91], the axion acquires a mass

$$
m_{a} \simeq 6 \cdot 10^{-6} \mathrm{eV}\left(\frac{10^{12} \mathrm{GeV}}{f_{a} / \mathcal{C}}\right)
$$

and its potential is given by

$$
V(a)=m_{u} \Lambda_{Q C D}^{3}\left[1-\cos \left(\frac{a}{f_{a} / \mathcal{C}}\right)\right],
$$

with $m_{u}$ being the up-quark mass and $\Lambda_{Q C D}$ being the QCD characteristic scale (see [98] for a review about axions in particle physics and [101] about the effects of QCD axions and other pseudoscalar fields in cosmology).

Depending on the decay constant $f_{a}$, the axion mass ${ }^{m}$ can be very light and stable, thus being an excellent candidate for dark matter [106-108]. Nowadays, a reliable mass range for ALPs (Axion-Like-Particles) is [9]

$$
10^{-33} \mathrm{eV} \leq m_{a} \leq 10^{-18} \mathrm{eV}
$$

where the lower bound comes from the Hubble constant and reflects constraints on axion dark energy, while the upper bound is related to cosmological structure formation. In fact, QCD axions are not the only axion-like fields which can be motivated by high energy physics, and different ALPs with very different masses

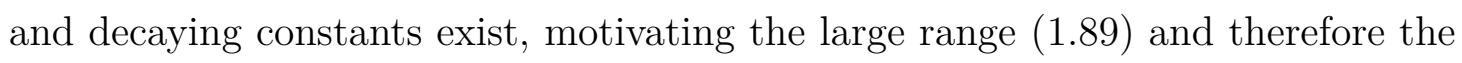
possibility to relate axions with different physical phenomena, such as dark energy, dark matter and inflation.

In addition to the QCD axions, it is important to mention the String Axiverse [97], namely the large class of pseudoscalar fields associated with compactified spatial dimensions in supergravity/string theory: for excellent reference books about

\footnotetext{
${ }^{1}$ The Peccei-Quinn mechanism we have described is the basic model to introduce an axion field solving the CP problem. However, when extra fields (quarks, Higgs) are taken into account, more specific axion models are required; among these, we only mention KSVZ [1122, 103] and DFSZ [1144, 111.5]
} 
strings, superstrings and M-theory, see [10.9-ए3]. Without entering into details, such axion fields arise as the Kaluza-Klein zero modes [66, 67] of the antisymmetric tensors contained in the supergravity description of string theory [14, 115], namely they are originated by the low energy limit of a higher-dimensional theory with compactified dimensions. As Kaluza-Klein zero modes, such axions are massless, but they can acquire mass through non-perturbative effects (such as instantons) as it happens for the QCD axion. However, "stringy" axions can have very different masses compared with the QCD ones, thus being associated with different physical phenomena. For more details about axions in string theory, the reader can refer to [96, 97].

Remarkably, axions can also play a crucial roll during inflation ([16-18]), a period of exponentially accelerated expansion of the Universe during its earliest phase. During the inflationary epoch, axions can both act as spectator fields (as cold dark matter candidates) or as the unstable, slow-rolling field driving inflation. In fact, in the simplest scenario, inflation is driven by a single, decaying field called inflaton $(\phi)$ which slowly rolls in the minimum of its potential $V(\phi)$. Once the minimum is reached, the inflaton begins to oscillate around the stable position, and it decays into radiation in a process known as reheating, which ends the inflationary epoch. If axions are stable fields (like the QCD ones and all the dark matter candidates), they cannot act as the inflaton, but they rather are spectator fields which populate the background and interact during the reheating phase. On the other hand, if we consider an unstable axion, that could act as the inflaton, being the rolling field driving the accelerated expansion of the Universe. In this scenario, the inflaton potential must be very flat compared to the other physical scales in play, in order to have the expansion and the size of the Universe at the end of the inflation compatible with observations and particle physics evidences. Such flat potential determines the slow rolling of the inflaton towards its minimum, and it determines the so-called slow roll parameters:

$$
\epsilon=\frac{M_{P l}^{2}}{2}\left(\frac{V^{\prime}}{V}\right)^{2}, \quad \quad \eta=M_{P l}^{2} \frac{V^{\prime \prime}}{V}
$$


which have to be very much smaller than unity. As an example, one of the simplest scenario of inflation driven by an axion is the so-called Natural Inflation, where the inflaton/axion potential is

$$
V(a)=\Lambda_{a}^{4}\left[1 \pm \cos \left(\frac{a}{f_{a}}\right)\right] .
$$

However, the details of axion inflation are beyond the purpose of this work: for further examples and for a more exhaustive discussion about axions during inflation, the reader can refer to [91].

To recap, we have seen how axions are produced and motivated by many high energy physics theories, such as QCD and string theory, and how such axions can have very different masses and decaying constants depending on the parameters of the theory they are originated by. Consequently, axions can be related with many different physical systems and phenomena; in particular, in cosmology, we have mentioned how axions can be good candidates for cold dark matter models and dark energy, and under some specific assumptions even for inflation. For what concerns this work, we are interested in investigating how axions (or generic scalar/pseudoscalar fields) can interact with black holes, looking for specific phenomena and which could lead to distinctive and detectable effects. For a review about the interactions of QCD axions with black hole physics the reader can refer to [119, ए20], while we mention [121] for an exhaustive review about the axiverse and black holes physics. At the end, we mention [122] for a detailed analysis about the possibilities to reveal ultralight bosons through gravitational wave detections with LIGO and LISA detectors.

\subsection{Superradiance in a nutshell}

Superradiance is a quite old and generic concept, which occurs in very different physical systems. In this section, we will provide a minimal introduction to the superradiance process from a general point of view, while we will discuss specifically about black holes superradiance in the next section. For clear and exhaustive 
reviews on superradiance, the reader can refer to [12:3-125].

In physics, superradiance refers to the radiation enhancement effects which occur in different contexts and physical systems, such as quantum optics, classical and quantum mechanics, astrophysics and relativity.

The first idea of superradiance can be found back in the 1929, when Klein showed how quantum mechanics predicts the transmission of particles in classically forbidden regions [1:26]. In 1946, Ginzburg and Franck studied transition radiation [127], which lead to the discovery of an anomalous Doppler effect causing an excitation of the emitted radiation. Few years later, in 1954 Dicke firstly introduced the concept of superradiance, meant as a collective phenomena of radiation-enhancement processes allowed by coherence of emitters [128]. Moreover, in 1971 Zel'dovich found that it is possible to have an amplification of reflected radiation caused by radiation scattering off rotating absorbing surfaces [12.9]. This fact is nowadays known as rotational superradiance, and it is triggered when the incident radiation (assumed monochromatic with frequency $\omega$ ) satisfies the condition

$$
\omega<m \Omega,
$$

with $m$ being the azimuthal number with respect to the rotational axis and $\Omega$ being the angular velocity of the rotating body.

For an insight of the superradiance effect, let's consider the following example. In $(1+1)$ dimensions, let's consider a massless scalar field with charge $q$ coupled to an electromagnetic potential $A_{\mu}$. The equations of motion are simply described by the Klein-Gordon equation

$$
\square \Phi=0,
$$

where the gauge covariant derivative is defined as

$$
\nabla_{\mu}=\left(\partial_{\mu}-i q A_{\mu}\right)
$$


Let's consider a potential of the form $A^{\mu}=\left\{A_{0}(x), 0\right\}$ such that

$$
A_{0} \rightarrow 0 \text { when } x \rightarrow-\infty, \quad A_{0} \rightarrow W \text { when } x \rightarrow+\infty .
$$

For the scalar field, we use the ansatz

$$
\Phi(t, x)=e^{-i \omega t} f(x)
$$

which makes the Klein-Gordon equation separable and leads to the following equation of motion along the spatial direction:

$$
\frac{d^{2} f}{d x^{2}}+\left(\omega-q A_{0}\right)^{2} f=0
$$

If we consider a flux of particles coming from $-\infty$ and scattering off the potential barrier $W$ with incident, transmission and reflection amplitudes $\mathcal{J}, \mathcal{T}$ and $\mathcal{R}$ respectively, we find a solution $f_{S}(x)$ with asymptotic behaviour such that

$$
\begin{aligned}
& f_{\text {in }}(x)=\mathcal{J} e^{i \omega x}+\mathcal{R} e^{-i \omega x}, \quad x \rightarrow-\infty \\
& f_{\text {out }}(x)=\mathcal{T} e^{i k x}, \quad x \rightarrow+\infty
\end{aligned}
$$

where we have defined

$$
k= \pm(\omega-q W)
$$

The transmission and reflection coefficients depend on the specific form of the potential $A_{0}$, but together with the incident coefficient $\mathcal{J}$ they satisfy the following condition [125]:

$$
|\mathcal{R}|^{2}=|\mathcal{J}|^{2}-\frac{\omega-q W}{\omega}|\mathcal{T}|^{2} .
$$

From the last expression, it is clear that if the frequency $\omega$ satisfies the condition

$$
0<\omega<q V
$$

the reflected amplitude is greater than the incident one:

$$
|\mathcal{R}|>|\mathcal{J}|
$$


which means that the incident radiation has been amplified through the scattering with the potential $A_{0}$ : this fact is precisely what we call superradiant amplification, or just superradiance.

In 1971, Zel'dovich [12.9] showed that an analogous phenomenon happens when radiation is scattered off rotating absorbing surfaces, and the superradiance condition (‥100) becomes

$$
\omega<m \Omega \text {. }
$$

Interestingly, the latter scenario is quite a common one when dealing with rotating black holes. In fact, the event horizon acts as a perfect dissipative membrane [130], and the ergosphere contributes to the amplification of the scattered radiation through the extraction of rotational energy from the black hole.

In the next section, we will see in more details how superradiance is related with black hole physics, and why it has become a very hot topic in modern astrophysics.

\subsection{Black holes part 2: no-hair theorem}

In modern astrophysics, one of the most interesting and still mysterious phenomena is the emission of relativistic jets from astrophysical objects, such as active galactic nuclei (AGNs). Although a clear and full comprehension of such mechanisms has not been achieved yet, they are commonly believed to be the result of some energy extraction mechanism. In this sense, superradiant enhancement could be one of the possible origins of some relativistic jets, and for this reason it has become a very popular and studied topic among the astrophysical scientific community. In particular, since the most powerful jets are seen in AGNs, great interest has been aroused in black hole superradiance. Based on the work done by Zel'dovich on rotational superradiance [12.9], starting from the Seventies many authors (Hawking, Teukolski, Press, Unruh, Blandford and Znajek, etc.) intensively worked on superradiance dealing with rotating black holes.

Nowadays, black holes superradiance can naturally be derived from thermodynamics arguments. As we have already seen, the first law of black holes thermodynam- 
ics is encoded in the Smarr formula

$$
\delta M=\frac{\kappa}{2} \delta A_{H}+\Omega_{H} \delta J+\psi_{H}^{Q} \delta Q+\psi_{H}^{P} \delta P
$$

where $M$ is the ADM mass, $\kappa$ the surface gravity, $\Omega_{H}$ the angular velocity of the black hole, $J$ the angular momentum, $Q$ and $P$ are the electric and the magnetic charges respectively and $\psi_{H}^{Q, P}$ are the electric and magnetic potentials respectively, with all quantities evaluated at the horizon.

For simplicity, let's start discussing the case of a rotating, neutral $(Q=P=0)$ black hole. If we consider a wave with frequency $\omega$ and azimuthal number $m$, the ratio of angular momentum to energy is [125]

$$
\frac{L}{E}=\frac{m}{\omega}
$$

and after scattering with the black hole the variation of angular momentum is

$$
\frac{\delta J}{\delta M}=\frac{m}{\omega}
$$

Plugging it into the Smarr formula we get

$$
\left(\omega-m \Omega_{H}\right) \delta M=\frac{\omega \kappa}{2} \delta A_{H}
$$

or equivalently

$$
\delta M=\frac{\omega \kappa}{2} \frac{\delta A_{H}}{\left(\omega-m \Omega_{H}\right)} .
$$

Extracting energy from the black hole means $\delta M<0$; recalling that the second law of black hole thermodynamics states that $\delta A_{H}>0$, the condition to extract energy from the black hole and hence to have superradiance is

$$
\omega-m \Omega_{H}<0
$$

In this case, rotational energy is extracted from the black hole and transferred to the reflected wave. However, as we will now show, it is also possible to extract energy from charged, non rotating black hole. 
Let's now consider the case of an electrically charged and non rotating black hole $(J=P=0)$ interacting with a monochromatic wave of frequency $\omega$ and charge $q$. Analogously to the rotating case, the ratio of charge to energy of the wave is given by

$$
\frac{Q}{E}=\frac{q}{\omega},
$$

and hence the interaction with the black hole causes a variation of the black hole charge

$$
\frac{\delta Q}{\delta M}=\frac{q}{\omega}
$$

Again, after plugging it into the Smarr formula we obtain

$$
\delta M=\frac{\omega \kappa}{2} \frac{\delta A_{H}}{\left(\omega-q \psi_{H}^{Q}\right)},
$$

and from the fact that $\delta A_{H}>0$ we get the condition for superradiance for a charged black hole,

$$
\omega-q \psi_{H}^{Q}<0,
$$

where the energy extracted is the black hole charge.

Generalising to the case of a rotating and charged black hole, the critical superradiance frequency is

$$
\omega_{c}=m \Omega_{H}+q \psi_{H}^{q},
$$

where $q$ is a generic charge and $\psi_{H}^{q}$ is the black hole's corresponding potential.

An interesting phenomenon related with black holes superradiant amplification is the so-called superradiant instability, which eventually leads to black hole bombs [131-[33]. Such instability is caused by the confinement of an exponentially increasing amount of energy in the black hole's surroundings; when the confined energy reaches the threshold level, a large amount of the confined energy is rapidly released: thus the evocative name "black hole bomb". Although at first sight it seems a simplistic or even idealistic problem, physical configurations comparable to the one just described can be actually achieved in the surroundings of rotating black holes [134-[36].

To understand it, let's consider the case of a scalar wave scattering off a rotating 


\section{INTRODUCTION AND THEORY REVIEW}

(Kerr) black hole. As we have described in the previous paragraph, depending on the black hole's angular velocity and on the scalar's frequency the superradiance

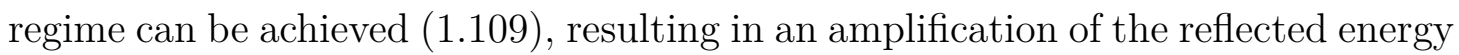
at the expense of a reduction of the black hole's rotational energy.

So, let's now assume the superradiance is triggered. If we also assume the existence of a perfectly reflecting screen (like a mirror) around the black hole and outside the ergosphere, a wave reflected and enhanced by scattering off the event horizon is then again totally reflected by the surrounding screen, causing the wave to be trapped between the horizon and the screen: in such a region, the scalar's energy density is therefore confined. Furthermore, since the "natural mirror" surrounding the black hole causes a total reflection of the superradiant waves back to the event horizon, these waves will be reflected and enhanced again, in a loop process. In such a loop, superradiant waves are trapped between the black hole's horizon and the mirror, being amplified after each scattering with the horizon; in this way, the confined energy exponentially increases, finally leading to an instability which causes a violent burst of energy which could be in principle observable [1:31-[1:33]. Moreover, the superradiant instability and the extraction of rotational energy from a spinning black hole can be related to the black holes populations in the Regge plane [121] , namely a black holes mass-spin diagram. In fact, since superradiance is triggered above a certain threshold mass (depending on the black hole and the scalar field masses) which is usually reached through accretion mechanisms, once the superradiant regime is achieved and rotational energy is extracted the black hole slows down, until the superradiance is exhausted (namely the superradiant regime is no longer satisfied). At this point, the black hole can accrete new mass, eventually undergoing to a new superradiant phase, then losing rotational energy and slowing down again and so on and so forth. Consequently, black holes superradiance affects the population in the Regge plane, since supermassive, extremely fast rotating black holes are predicted to not exist (for a more exhaustive discussion about superradiance predictions in the Regge plane, see chapter 6 of [प25]). Thus, the natural arising question is: do black hole bombs and screening mirrors exist in nature? Interestingly, for massive scalar fields surrounding rotating black holes a "natural mirror" mechanism is provided by the Yukawa potential of the scalar field, which naturally acts like a perfect mirror reflecting the low frequency modes 
[137, [38]. On the other hand, black hole bombs can also develop for massless scalar fields, and it is therefore necessary to consider other mechanisms to provide the mirror reflection, such as high-density matter in the black hole's surroundings [1.34, [.3.9, [40]]. Remarkably, since superradiance can be triggered even in the case of non rotating but charged black holes, charged black holes bombs can detonate as well, providing the existence of a confining, screening mechanism close to black hole's horizon. Without going into details about the physical nature of the surrounding screen, on the base of the work done in [141, 142] we will soon show how the superradiant instability for a charged black hole can end in a stable, static, hairy configuration.

It is important to have a closer look at this physical system because, as we will see soon, it can naturally provide an example of black hole with scalar hair, in contrast to the no-hair theorem originally established by Israel [143] for the Schwarzschild black hole and subsequently refined and extended to the case of rotating and charged black holes. As simple as it sounds, the no-hair theorem states that, in General Relativity, all the black hole solutions of the EinsteinMaxwell action are described by only three observable classical parameters: mass, electromagnetic charge and angular momentum, and all the other parameters (information) describing the matter and the energy of the black hole are lost inside the event horizon [144]. In this sense, the black holes have no-hair, namely they do not have any physical parameter observable at infinity (other than their mass, charge and angular momentum). However, since all theorems are valid as long as their assumptions are, the validity of such a strong theorem is based on two crucial hypotheses/assumptions: the General Relativity gravitation theory and the Einstein-Maxwell action. Thus, since the late Eighties a lot of counterexamples have been proposed, showing the possibility of existence of black holes with hair when some of the no-hair theorem hypotheses are relaxed; in particular, the very first hairy black holes solutions were found in the context of the Skyrme model [145] and Yang-Mills theory [146, 147]. Following these, several examples of black holes with hair have been found; among these, we mention [148-152]; for a brief summary of some important hairy black hole solutions found in the past years look at [1.5.3, 1.54]. 


\section{INTRODUCTION AND THEORY REVIEW}

As far as we are concerned in this work, in the context of modified gravity theories with extra fields non-minimally coupled with gravity and/or electromagnetism, several "not bald" black holes (i.e. they carry observable charge other than their mass, electromagnetic charge and angular momentum ) have been found [155-162].

Let's now go back to our example of static, charged black hole coupled with a charged scalar field. The gravity-electromagnetic-scalar field system is described by the action

$$
S=\int d^{4} x \sqrt{-g}\left[\frac{R}{4}-\frac{1}{4} F_{\mu \nu} F^{\mu \nu}-\frac{1}{2} g^{\mu \nu} D_{(\mu}^{*} \psi^{*} D_{\nu)} \psi\right]
$$

where $D_{\mu}=\nabla_{\mu}-i q A_{\mu}$ is the gauge derivative, $\nabla_{\mu}$ is the covariant derivative and the symmetric/antisymmetric combinations are defined as

$$
v_{(\mu \nu)}=\frac{1}{2}\left(v_{\mu \nu}+v_{\nu \mu}\right), \quad v_{[\mu \nu]}=\frac{1}{2}\left(v_{\mu \nu}-v_{\nu \mu}\right) .
$$

The gravity, electromagnetic and scalar field equations obtained from the action (‥15) can be neatly written as:

$$
\begin{aligned}
& G_{\mu \nu}=2\left(T_{\mu \nu}^{F}+T_{\mu \nu}^{\psi}\right), \\
& \nabla_{\mu} F^{\mu \nu}=J^{\nu}, \\
& D_{\mu} D^{\mu} \psi=0,
\end{aligned}
$$

with

$$
\begin{aligned}
T_{\mu \nu}^{F} & =F_{\mu \alpha} F_{\nu}^{\alpha}-\frac{1}{4} g_{\mu \nu} F^{\alpha \beta} F_{\alpha \beta}, \\
T_{\mu \nu}^{\psi} & =D_{(\mu}^{*} \psi^{*} D_{\nu)} \psi-\frac{1}{2} g_{\mu \nu}\left[g^{\alpha \beta} D_{(\alpha}^{*} \psi^{*} D_{\beta)} \psi\right], \\
J^{\nu} & =\frac{i q}{2}\left[\psi^{*} D^{\nu} \psi-\psi\left(D^{\mu} \psi\right)^{*}\right] .
\end{aligned}
$$

If we consider a small-amplitude scalar field with negligible backreaction on the background geometry, in Boyer-Lindquist coordinates the spacetime is described by the standard Reissner-Nordström metric:

$$
d s^{2}=-f_{R N} d t^{2}+f_{R N}^{-1} d r^{2}+r^{2} d \theta^{2}+r^{2} \sin ^{2} \theta d \varphi^{2}
$$


where

$$
f_{R N}=1-\frac{2 M}{r}+\frac{Q^{2}}{r^{2}}
$$

and the electromagnetic potential is

$$
A_{\mu}=\left\{-\frac{Q}{r}, 0,0,0\right\}
$$

with $Q$ being the electric charge.

For the scalar field, let's assume a small-amplitude perturbation, monochromatic with frequency $\omega$ and spherically-symmetric:

$$
\psi(t, r)=\frac{\phi(r)}{r} e^{-i \omega t}
$$

Introducing for convenience the tortoise coordinate $r_{*}$, the equation of motion of the scalar field becomes

$$
\frac{d^{2} \phi}{d r_{*}^{2}}+\left[\left(\omega-\frac{q Q}{r}\right)^{2}-\frac{f_{R N}}{r} \frac{d f_{R N}}{d r}\right] \phi=0 .
$$

At this point, we have to impose adequate boundary conditions. In this case, we require that the scalar field is purely in-going on the event horizon; moreover, assuming the existence of a perfectly reflecting mirror located at $r=r_{M}$, we also require a vanishing scalar field at the mirror's location:

$$
\phi(-\infty) \sim e^{-i \omega r_{*}}, \quad \phi\left(r_{M}\right)=0 .
$$

These boundary conditions determine a discrete spectrum of states with frequencies $\omega_{n}$. In general, the eigenvalues $\omega_{n}$ are complex: a positive imaginary part corresponds to an exponentially growing mode, while a negative imaginary part corresponds to an exponentially decaying one. The index $n$ denotes the number of nodes of the corresponding mode in the region $r<r_{M}$; thus, the $n=0$ mode has the mirror located at the first zero of the scalar field, the $n=1$ one at the second zero, the $n=2$ mode at the third one and so on and so forth [132, 141]]. In [14I], it has been found that configurations with the mirror placed close to the black hole horizon admit only $n=0$ decaying modes, while configurations with the 
mirror placed far from the horizon admit exponentially growing $n=0$ modes, thus leading to a superradiant instability. ${ }^{\boxplus}$. To investigate if any hairy configuration exist, the following ansatz for the metric is used:

$$
d s^{2}=-f(r) h(r) d t^{2}+f^{-1}(r) d r^{2}+r^{2} d \theta^{2}+r^{2} \sin ^{2} \theta d \varphi^{2}
$$

with

$$
f(r)=1-\frac{2 m(r)}{r}
$$

and where $m(r)$ is the effective mass within the sphere of radius $r$.

The scalar field is assumed to be real and, thanks to gauge freedom, it can be chosen time independent: $\psi(t, r)=\phi(r)$. Similarly, exploiting the gauge freedom the electromagnetic potential can be chosen in the form: $A_{\mu}=\left\{A_{0}(r), 0,0,0\right\}$. The equations of motion for $\left\{f, h, A_{0}, \phi\right\}$ now read

$$
\begin{aligned}
& h^{\prime}=2 r\left[\left(\frac{q A_{0} \phi}{f}\right)^{2}+h\left(\phi^{\prime}\right)^{2}\right], \\
& 2\left(A_{0}^{\prime}\right)^{2}=-\frac{2}{r}\left[f^{\prime} h+\frac{1}{2} f h^{\prime}+\frac{h}{r}(f-1)\right], \\
& 0=f A_{0}^{\prime \prime}+\left(\frac{2 f}{r}-\frac{f h^{\prime}}{2 h}\right) A_{0}^{\prime}-q^{2} \phi^{2} A_{0}, \\
& 0=f \phi^{\prime \prime}+\left(\frac{2 f}{r}+f^{\prime}+\frac{f h^{\prime}}{2 h}\right) \phi^{\prime}+\frac{\left(q A_{0}\right)^{2}}{f h} \phi,
\end{aligned}
$$

where the prime' denotes a variation with respect to $r$.

Again, we have to impose adequate boundary conditions on the horizon (see [141] for a discussion on the following boundary conditions):

$$
f\left(r_{H}\right)=0, \quad f^{\prime}\left(r_{H}\right)>0, \quad A_{0}\left(r_{H}\right)=0, \quad h\left(r_{H}\right)=1,
$$

in addition with the boundary condition at the mirror location

$$
\phi\left(r_{M}\right)=0
$$

\footnotetext{
${ }^{1}$ It has been found that superradiant instability happens for both massless and lightly massive scalar fields.
} 
The set of equations (‥27) together with the corresponding boundary conditions can be analytically solved with a Taylor series expansion near the black hole horizon [141], with all the functions depending just on a family of three parameters: the scalar charge $q$, the scalar field magnitude on the horizon $\phi\left(r_{H}\right)$ and the electric field on the horizon $A_{0}^{\prime}\left(r_{H}\right)$. Such results are also confirmed by numerical integrations, which show that hairy configurations are supported depending on the parameters $\left\{q, \phi\left(r_{H}\right), A_{0}^{\prime}\left(r_{H}\right)\right\}$ [14I] . Solutions with $\phi\left(r_{H}\right)=0$ correspond to the GR Reissner-Nordström ones, while uncharged solutions $\left(A_{0}^{\prime}=0\right.$ or $q=0$ ) cannot exist, since the scalar field does not develop nodes and do not vanish on the mirror.

To study the stability of such hairy configurations, it is necessary to consider a linear perturbation of a non-vacuum hairy solution.

Denoting with $\left\{\bar{f}(r), \bar{h}(r), \bar{A}_{0}(r), \bar{\phi}(r)\right\}$ the static, equilibrium functions which satisfy the equations (․27), we write the following time-dependent perturbations:

$$
\begin{aligned}
f & =\bar{f}(r)+\delta f(t, r), \\
h & =\bar{h}(r)+\delta h(t, r), \\
A_{0} & =\bar{A}_{0}(r)+\delta A_{0}(t, r), \\
\psi & =\bar{\psi}(r)+\delta \psi(t, r),
\end{aligned}
$$

where the only complex quantity is the scalar field perturbation $\delta \psi(t, r)$ with corresponding complex frequency $\omega$. Similarly to (ए..28), after imposing boundary conditions at both the event horizon and the mirror location all the perturbations (tensor, vector and scalar) appear to decay, making the solution stable [141].

Although we did not enter into much detail, in addition to being a clear example of hairy black hole with a non trivial scalar hair supported, the procedure described above to find the black hole solution and stability is very similar to the one we will use in the next sections. At this point, it should be clear that hairy black holes are actually plausible physical configurations, which can naturally develop even from simple physical systems. In particular, concerning this work we are interested in studying black hole configurations with scalar hair, since scalar fields non minimally coupled with gravity and matter are currently valid candidates for 


\section{INTRODUCTION AND THEORY REVIEW}

both dark energy and dark matter. Thus, let's now have a closer look to black holes with scalar hair. As we have already discussed, the surprisingly simplicity of black holes structure in GR is described by the No-hair theorem; however, as we have pointed out, the validity and the strength of the theorem relies on the validity of the assumptions, and relaxing any of them results in a natural circumvention of the theorem. In particular, being based on the uniqueness of the Schwarzschild and the Kerr families of black holes, the no-hair theorem is consequently based on the assumptions of stationarity and asymptotic flatness of the geometry. Thus, it could be interesting to investigate whether the theorem could be extended to a larger class of black hole solutions (satisfying stationarity and asymptotic flatness) coming from gravity theories different from GR (look for example at [16.3] for non spinning black holes). Among these, we are particularly interested in scalar-tensor ones. For such theories, ulterior assumptions on the scalar field symmetries and on the matter sector are often required, which therefore leads to the possibility of avoiding the theorem by breaking any of its assumptions. [164]. For example, as it happens for stars and compact objects, in the presence of external matter the black hole can develop a scalarization, namely a spontaneous accretion of a non trivial scalar field profile around the black hole which ends to be stationary, and hence a possible final, stable, hairy black hole geometry [1.35]. In their paper [1.35], the authors show how the final state of the black hole depends on the nature of the scalar field it is coupled with; in particular, depending on the scalar field's potential, its effective mass can be positive or negative, affecting the stability of the configuration. If the effective mass is negative, the system is affected by a tachyonic instability which leads to a spontaneous scalarization, resulting in a stable hairy configuration; on the other hand, if the effective mass is positive and the geometry admits an ergoregion, depending on the matter configuration and on the specific parameters of the theory it is possible to trigger the superradiant instability. Then, depending on the frequency of the scalar field modes and on the black hole mass and angular velocity, the superradiant instability can lead to a black hole bomb or to long lived stationary scalar field configurations - known as scalar clouds - around the black hole [135, 165]. 
Following the work done in [135], we now show how the spontaneous scalarization or the superradiant/scalar cloud configurations are developed. The results we will provide in the following are therefore well known in the literature, but we believe it is a useful, didactic introduction for a reader unfamiliar with such topic. On the other hand, if the reader is already familiar or they are not interested into more technical details, they can directly move to the next chapter.

As we have already discussed in the modified gravity section, scalar-tensor theories of gravity can be described, in the Jordan frame, by the following generic action $[68]$ :

$$
S=\frac{1}{4} \int d^{4} x \sqrt{-g}\left[F(\Phi) R-Z(\Phi) g^{\mu \nu} \partial_{\mu} \Phi \partial_{\nu} \Phi-V(\Phi)\right]+S\left(\psi_{m} ; g_{\mu \nu}\right)
$$

where $g_{\mu \nu}$ is the spacetime metric, $R$ is the associated Ricci scalar, $\Phi$ is the scalar field and $S\left(\psi_{m} ; g_{\mu \nu}\right)$ is the matter action, with $\psi_{m}$ denoting the matter fields minimally coupled to the background metric $g_{\mu \nu}$. The specific shape of functions $F(\Phi), Z(\Phi)$ and $V(\Phi)$ determine the specific theory within the family of theories. Performing the following transformations

$$
\tilde{g}_{\mu \nu}=F(\Phi) g_{\mu \nu}, \quad \phi(\Phi)=\int d \Phi\left[\frac{3}{4} \frac{F^{\prime}(\Phi)^{2}}{F^{2}(\Phi)}+\frac{1}{2} \frac{(\Phi)}{F(\Phi)}\right]^{1 / 2}
$$

and defining

$$
A(\phi)=\frac{1}{F^{1 / 2}(\Phi)}, \quad W(\phi)=\frac{V(\Phi)}{F^{2}(\Phi)}
$$

we can recast the action $\square . J \mathbb{1}$ in the Einstein frame

$$
S=\int d^{4} x \sqrt{-\tilde{g}}\left[\frac{\tilde{R}}{4}-\frac{1}{2} \tilde{g}_{\mu \nu} \partial^{\mu} \phi \partial^{\nu} \phi-\frac{W(\phi)}{4}\right]+S\left(\psi_{m} ; A^{2}(\phi) \tilde{g}_{\mu \nu}\right)
$$

Assuming a regular behaviour of the potentials $A(\phi)$ and $W(\phi)$ around $\phi \sim \phi_{0}$ 


\section{INTRODUCTION AND THEORY REVIEW}

and expanding the fields equations to first order in $\psi=\phi-\phi_{0}$, we get [1.35]

$$
\begin{aligned}
\tilde{G}_{\mu \nu}= & +2\left(\tilde{T}_{\mu \nu}+\partial_{\mu} \phi_{0} \partial_{\nu} \phi_{0}-\frac{\tilde{g}_{\mu \nu}}{2} \partial_{\mu} \phi_{0} \partial^{\mu} \phi_{0}\right)-\frac{\tilde{g}_{\mu \nu}}{2} W_{0} \\
& +2\left(\partial_{\mu} \phi_{0} \partial_{\nu} \psi+\partial_{\mu} \psi \partial_{\nu} \phi_{0}-\frac{\tilde{g}_{\mu \nu}}{2} \partial_{\mu} \phi_{0} \partial^{\mu} \psi\right)-\psi \frac{\tilde{g}_{\mu \nu}}{2} W_{1}, \\
\tilde{\square} \phi_{0}+\tilde{\square} \psi= & -\frac{A_{1}}{A_{0}} \tilde{T}+\frac{W_{1}}{4}+\psi \frac{W_{2}}{2}+\psi \tilde{T}\left(\frac{A_{1}^{2}}{A_{0}^{2}}-2 \frac{A_{2}}{A_{0}}\right)
\end{aligned}
$$

with

$$
\tilde{T}_{\mu \nu}=-\frac{2}{\sqrt{-\tilde{g}}} \frac{\delta S}{\delta \tilde{g}^{\mu \nu}}
$$

and where $A_{i}$ and $W_{i}$ are the $i^{t h}$-coefficient of the Taylor expansions around $\psi$ for $A(\phi)$ and $W(\phi)$, respectively.Since we are interested in asymptotic flat solutions, we can set $W_{0}=W_{1}=0$. Looking at equations (‥3.5), it is clear that in the presence of matter the GR solution $\phi_{0}=$ const is not a solution of the field equations, unless $A_{1}=0$. Thus, if $A_{1} \neq 0$, the black hole solution necessary develops a scalar hair, since the constant (trivial) scalar field configuration (which is a GR solution) is not a solution anymore and the scalar field must acquire a non trivial profile. On the other hand, if $A_{1}=0$ any solution with constant scalar field satisfies the field equations (ㄷ.5.3.): anyway, it is possible that perturbative effects due to the surrounding matter allow the formation of a supported non trivial scalar hair around the black hole. To show this, let's assume $A_{1}=0$ and we are left with the following Klein-Gordon equation (‥J35):

$$
\left[\tilde{\square}-\frac{W_{2}}{2}+\frac{2 A_{2}}{A_{0}} \tilde{T}\right] \psi=0 .
$$

From the last equation, we can define an effective mass

$$
\mu_{e}^{2}(r)=\frac{W_{2}}{2}-\frac{2 A_{2}}{A_{0}} \tilde{T}
$$

and simply rewrite equation (ㄷ.57) as Klein-Gordon equation with mass term:

$$
\left[\tilde{\square}-\mu_{e}^{2}(r)\right] \psi=0
$$


By definition, the coefficient $W_{2}$ is the coefficient of the quadratic term in the Taylor expansion of the potential $W(\phi)$, and it is therefore associated with the standard mass of the scalar field; since we are interested in ultralight scalar field with Compton length much larger than the black hole's Schwarzschild radius, we can effectively consider the scalar field as massless, and hence setting $W_{2}=0$ without loosing too much of generality. Thus, from the expression of the effective mass $\mu_{e}^{2}(r)$ we see that depending on the sign of $A_{2} / A_{0}$ and $\tilde{T}$, the effective mass could either be positive or negative. As we will now show, depending on the positivity/negativity of the effective mass the black hole could trigger two very different and interesting phenomena: spontaneous scalarization (when the mass is negative) or superradiant instability (when the mass is positive), which can eventually lead to the formation of stationary scalar clouds around the black hole. In any case, the presence of the scalar field really affects the final configuration, leading to qualitatively different configurations from GR.

\section{Spontaneous scalarization}

Let's start with the case of a negative effective mass $\mu_{e}$, which as we will now show leads to a spontaneous scalarization.

We consider a spherically symmetric background described by the Schwarzschild metric in Boyer-Lindquist coordinates; for the scalar field, assuming the separability of the variables we use the following ansatz:

$$
\psi(t, r, \theta, \varphi)=\sum_{l m} \frac{\psi_{l m}(r)}{r} Y_{l m}(\theta, \psi) e^{-i \omega t}
$$

which plugged into the equation (ㄷ.J3) gives the equation of motion of the radial component:

$$
\frac{d^{2} \psi_{l m}}{d r_{*}^{2}}+\left[\omega^{2}-\mathcal{V}(r)\right] \psi(r)=0,
$$

where we have introduced the tortoise coordinate $r_{*}$

$$
d r=\left(1-\frac{2 M}{r}\right) d r_{*}
$$


and we have defined the effective potential

$$
\mathcal{V}(r)=\left(1-\frac{2 M}{r}\right)\left[\frac{l(l+1)}{r^{2}}+\frac{2 M}{r^{3}}+\mu_{e}^{2}(r)\right] .
$$

As boundary conditions, as usual we impose the scalar field to be in-going on the horizon and purely out-going at spatial infinity:

$$
\psi_{H} \sim e^{-i \omega r_{*}}, \quad \psi_{\infty} \sim e^{+i \omega r_{*}}
$$

Such boundary conditions determine a discrete set of eigenvalues for the complex frequency $\omega$; stable, decaying modes have a negative imaginary component $\operatorname{Im}(\omega)<0$, while eigenmodes with a positive imaginary component $\operatorname{Im}(\omega)>0$ are exponentially growing and hence unstable; given the potential $\mathcal{V}(r)$, a sufficient condition to get unstable modes is [166]

$$
\int_{2 M}^{\infty} \frac{\mathcal{V}}{f} d r<0
$$

which can be written in terms of the energy-stress momentum tensor using (․143) and (…38):

$$
2 \frac{A_{2}}{A_{0}} \int_{2 M}^{\infty} \tilde{T} d r>\frac{2 l(l+1)+1}{4 M} .
$$

At this point, to find the eigenvalues spectrum one should specify the matter configuration $\tilde{T}_{\mu \nu}$; in [1.35] two explicit solutions for appropriate, simple choices of $\tilde{T}_{\mu \nu}$ have been found, to show that spontaneous scalarization occurs above a threshold effective mass $\mu_{T}$. Remarkably, the presence of spontaneous scalarization above a certain threshold mass seems to be a quite generic phenomenon shared by many different physical systems. In particular, it has been shown that these results hold for spherically symmetric background more general than the Schwarzschild one, making this approach available for a larger class of black holes surrounded by matter configurations. In addition, in [135] has been determined the final, stationary, spherically symmetric solution for a thin matter shell configuration 
around a Schwarzschild black hole:

$$
d s^{2}=-\left(1-\frac{2 M}{r}+F(r)\right) d t^{2}+\frac{d r^{2}}{1-2 M / r+G(r)}+r^{2} d \theta^{2}+r^{2} \sin ^{2} \theta d \varphi^{2}
$$

where $\psi(r), F(r)$ and $G(r)$ are such that inside the shell we have

$$
\psi=\text { const }, \quad F(r)=G(r)=\frac{\text { const }}{r},
$$

while outside the shell we have

$$
\begin{aligned}
& \psi^{\prime}(r)=\frac{\Sigma}{r(r-2 M)}, \\
& F=-\frac{\Sigma^{2}}{2 M^{2} r}\left[2 M+(r-M) \log \left(1-\frac{2 M}{r}\right)\right], \\
& G=\frac{\Sigma^{2}}{2 M r} \log \left(1-\frac{2 M}{r}\right) .
\end{aligned}
$$

This is a black hole solution carrying an extra independent charge, namely a primary $^{\mathbb{m}}$ scalar hair; in fact, at large distances the scalar field goes like

$$
\psi \sim \frac{\Sigma}{r}
$$

with $\Sigma$ being the scalar charge completely determined by the matter content. Interestingly, for theories such that $A_{1} \neq 0$, the scalar charge also contributes to the black hole total mass:

$$
-g_{t t}=1-\frac{2 M-A_{1} \Sigma}{r}+\frac{A_{1}^{2} \Sigma^{2}-2 A_{1} M \Sigma+2 A_{2} \Sigma^{2}}{r^{2}} .
$$

and therefore also the event horizon radius depends on the scalar field charge $\Sigma$.

\footnotetext{
${ }^{1}$ We will discuss about the difference among primary and secondary scalar hair in the next chapters.
} 


\section{INTRODUCTION AND THEORY REVIEW}

To summarize, spherically symmetric black hole backgrounds in scalar-tensor theories with matter content can naturally develop and support scalar hair, which is consequently fully determined by the specific matter configuration and not by the black hole parameters, being in this sense a primary hair. Since this is a generic effect which also occurs for theory admitting GR solutions, it could be in principle used as a smoking gun to detect non GR solutions.

\section{Superradiant instability}

In a different scenario, when the effective mass $\mu_{e}$ defined as in ([. .38 ) is positive, the scalar field can spontaneously trigger a superradiant instability and eventually settle down into stable, stationary scalar clouds surrounding the black hole. As in the case of massive scalar fields around rotating black hole with an ergosphere (or even static charged black holes), superradiant enhancement can extract rotational energy (or charge) from the black hole and end in a final state with possible supported hair ([141], [142], [157]). For the scalar field $\psi$ and the effective mass $\mu_{e}$ we use the following ansatze:

$$
\begin{aligned}
& \psi=e^{-i \omega t+i m \varphi} \psi(r) S(\theta) \\
& \mu_{e}^{2}(r)=\mu_{0}^{2}+\frac{h(\theta)+k(r)}{r^{2}+a^{2} \cos ^{2} \theta}
\end{aligned}
$$

where $\mu_{0}$ is the canonical mass of the scalar field and $a$ is the rotational parameter $a=J / M$. Thus, it is possible to separate the radial and the angular equations, and we get [167]:

$$
\begin{aligned}
& \frac{d}{d r}\left(\Delta \frac{d \psi(r)}{d r}\right)+\left[\frac{\omega^{2}\left(r^{2}+a^{2}\right)^{2}-4 a M r m \omega+a^{2} m^{2}}{\Delta}-k(r)-r^{2} \mu_{0}^{2}-\lambda_{l m}-a^{2} \omega^{2}\right] \psi(r)=0, \\
& \frac{1}{\sin \theta} \frac{d}{d \theta}\left(\sin \theta \frac{d S(\theta)}{d \theta}\right)+\left[a^{2}\left(\omega^{2}-\mu_{0}^{2}\right) \cos ^{2} \theta-\frac{m^{2}}{\sin ^{2} \theta}-h(\theta)+\lambda_{l m}\right] S(\theta)=0,
\end{aligned}
$$

where

$$
\Delta=r^{2}+a^{2}-2 M r
$$


and with $\lambda_{l m}$ being the separation constant coming from the regularity conditions imposed on the angular function $S(\theta)$. Obviously, to different choices of $h(\theta)$ and $k(r)$ correspond different models, which could in principle behave very different one from the other. However, even if a systematic and general study is still required, some common features appear to be shared by many different configurations [1:35. For example, as in the case of a massive scalar field around a rotating black hole screened by a perfect mirror, the unstable modes $\left(\omega_{I}>0\right)$ trigger superradiance when the condition $\omega<m \Omega_{H}$ is satisfied, with $\Omega_{H}$ being the black hole angular velocity. Moreover, an incident wave which satisfies superradiance condition and which therefore admits the following asymptotic behaviours

$$
\begin{array}{ll}
\mathcal{T} e^{-i\left(\omega-m \Omega_{H}\right) r_{*}} & r_{*} \rightarrow+\infty \\
e^{-i \omega r_{*}}+\mathcal{R} e^{i \omega r_{*}} & r_{*} \rightarrow-\infty
\end{array}
$$

where $\mathcal{T}$ and $\mathcal{R}$ are the renormalized transmission and reflection coefficients respectively, usually admits resonant frequencies in the amplification factor, i.e. frequencies such that $\mathcal{R}\left(\omega_{r}\right) \rightarrow \infty$. As pointed out in [135], the existence of modes such that $\omega_{R} \rightarrow \omega_{\text {res }}$ suggests the existence of very long-lived stationary modes, such that $\omega_{I} \ll \omega_{R}$, analogous to the resonant stationary modes for massive scalar perturbations of Kerr black holes [168]. In fact, when $\omega_{R} \sim \omega_{\text {res }}$, the wave at infinity is almost purely outgoing, and since $\omega_{I} \sim 0$ the mode is stationary, namely it is not decaying or growing; thus, in the surrounding of the black hole, there exist a "cloud" of non vanishing, stationary scalar field modes, whose frequencies correspond to the resonant frequencies of the superradiant gain factor.

\subsection{Summary}

To summarize, in this chapter we have introduced the basic aspects of black hole physics, modified gravity and axion cosmology necessary to understand the results discussed in the next chapters. Moreover, in section [.6] we have seen how the presence of scalar fields (and matter) in the surrounding of static/rotating and neutral/charged black holes can lead to configurations with a non trivial scalar 
profile supported. Depending on the mass and frequency of the scalar field, the black hole can trigger superradiance, eventually developing an instability and detonating a black hole bomb leading to a final hairy configuration or accreting a stationary scalar cloud in the black hole surroundings. Interestingly, in any scenario the presence of the scalar field leads to final configurations different and potentially distinguishable from the GR ones. On this basis, in the following chapters we will focus our attention on different systems with black holes in non standard GR theories, looking for potentially distinctive traits of the resulting black hole configurations. 


\section{Chapter 2}

\section{A new rotating black hole solution}

In this chapter, based on [169], we find an exact rotating black hole solution in a Generalised Proca theory of gravity. Exact solutions describing rotating black holes can offer important tests for alternative theories of gravity, motivated by the dark energy and dark matter problems. We present an analytic rotating black hole solution for a class of vector-tensor theories of modified gravity, valid for arbitrary values of the rotation parameter. The new configuration is characterised by parametrically large deviations from the Kerr-Newman geometry, controlled by non-minimal couplings between vectors and gravity. It has an oblate horizon in Boyer-Lindquist coordinates, and it can rotate more rapidly and have a larger ergosphere than black holes in General Relativity (GR) with the same asymptotic properties. We analytically investigate the features of the innermost stable circular orbits for massive objects on the equatorial plane, and show that stable orbits lie further away from the black hole's horizon with respect to rotating black holes in GR. We also comment on possible applications of our findings for the extraction of rotational energy from the black hole.

\subsection{Motivations and introduction}

The new era of gravitational wave astronomy opens new opportunities for investigating with great precision the physics and dynamics of extreme compact objects, 


\section{A NEW ROTATING BLACK HOLE SOLUTION}

such as black holes and neutron stars (see e.g. [[7/0]), and it will allow us to study for the first time the properties of fundamental interactions in a strong gravity regime, and to test theories of gravity that are alternative to Einstein's General Relativity (GR) [ 8$]$. Moreover, the lack of a fully understood and consistent theory of cosmological dark energy and dark matter keeps motivating the attempts to modify GR to extend it to a broader gravity theory. For example, it is important to explore the possibility to find theories admitting accelerating cosmological solutions with no need of a small positive cosmological constant (see e.g. [[7]] ). The theoretically most interesting frameworks include scenarios automatically equipped with screening features, as chameleon [56] or Vainshtein [54] mechanisms. Screening mechanisms are able to locally hide the effects of additional light degrees of freedom besides GR's spin-2 field, and reproduce the predictions of Einstein's theory of gravity in a weak-field, spherically symmetric regime: see [172] for a review. The study of the properties of black hole solutions in these scenarios can provide new strong gravity tests for these theories, possibly manifesting sizeable deviations from GR. In this chapter, based on [16.9], we focus on theories with additional degrees of freedom non-minimally coupled to gravity through derivative interactions, which are essential for an implementation of Vainshtein screening mechanism. In the scalar-tensor case, the prototypes for such set-up are Galileons [173] and Horndeski [174] theories. The study of spherically symmetric black hole solutions in these scenarios have lead to various interesting results, reviewed e.g. in [158]. Here we focus on vector-tensor versions of these theories, dubbed vector Galileons, or Generalised Proca [10, 175, ए76]. Various examples of static, spherically symmetric black hole configurations have been found, while the study of compact objects as neutron stars have been recently developed [161, 177-184]. Moreover, GWs detections [37] have confirmed that the majority of astrophysical black holes are spinning, setting the need of rotating black hole solutions in the contest of modified gravity theories, with the possibility to find new ways to test GR and other modified gravity theories.

In this work, we present and study examples of rotating black hole solutions with regular horizons for vector Galileons. Rotating black hole configurations which deviate from the Kerr family are hard to obtain in any theory of gravity, 
and only few examples of exact solutions are known in modified gravity frameworks. Solutions are known for scalar-tensor theories [185, 186], also with a complex scalar [157], and in the context of Einstein dilaton Gauss-Bonnet theories [187, 188]. Moreover, slowly rotating solutions in Horndeski theories are discussed in [18.9]: see [2] for a comprehensive review, and [1.90, [191] for useful parameterisations of possible deviations from the Kerr family of black holes in the context of arbitrary theories of gravity. Yet, given the fact that most astrophysical black holes are spinning, it is important to pursue the effort to determine and analyze explicit rotating black hole configurations in theories alternative to General Relativity. An additional theoretical reason to study rotating configurations is the fact that these objects break spherical symmetry (being at most axially symmetric). Hence they are an ideal set-up to start investigating screening mechanisms - as for example the Vainsthein mechanism - that are well studied and are known to be efficient only for spherically symmetric systems.

In this chapter, we determine exact solutions describing rotating black holes, by applying a disformal transformation [13, 1.92] on a version of the Kerr-Newman (KN) solution of the Einstein-Maxwell theory of gravity. The resulting configuration solves the equations of motion associated to a particular vector Galileon action, and it is parametrically different from a KN system. Having exact solutions allows us to analytically investigate distinctive properties of spinning black holes in our theory; first, we show that our configurations are characterized by three asymptotic charges, namely the black hole's mass, angular momentum, and vector charge. We then show that for a particular choice of the disformal transformation the black hole's horizon is oblate in Boyer-Lindquist coordinates, since its radial position depends on the polar angle. This is a feature that can make such black hole configuration distinguishable from KN solutions, whose horizon lies at constant value of the radius in such coordinate system. Moreover, the black hole's maximal spin can be parametrically larger than KN configurations, for the same values of the asymptotic charges. This solution also admits a 'massless' limit of black hole with zero mass, but with a vector charge which ensures the existence of a regular horizon. The study of equatorial circular trajectories admits an analytical treatment, and we show that probe massive objects can rotate faster than 


\section{A NEW ROTATING BLACK HOLE SOLUTION}

in the KN family of solutions. Quite remarkably, innermost stable circular orbits (ISCOs) lie further away from the black hole's horizon with respect to rotating black holes in GR, with possible applications for the extraction of rotational energy from the black hole.

This chapter is structured as follows. In section 2.2 we discuss the set-up of the modified gravity theory we study: we apply a disformal transformation to a $\mathrm{KN}$ black hole in order to generate a different black hole solution in an effective vector-tensor theory. In section 2.3 we go through a brief but necessary review of basic geometrical concepts such as singularities, killing vectors and horizons. In section 2.4 we discuss our results, i.e. an exact, rotating black hole solution in a vector-tensor theory. In particular, as it will be explained, this regular black hole solution comes from a particular choice for the applied disformal transformation, specifically discussed in subsection [2.4.2: for such solution, we also study the ISCO trajectories, since they could provide distinctive physical features distinguishable from GR. At the end, in section 2.5 we conclude with a short recap of the main results we found and we briefly discuss about possible applications and future developments for such results. Throughout the chapter, we use a mostly plus metric signature, and Weinberg's conventions for the Riemann and derived tensors: our results are expressed in natural units, setting $c=\hbar=G=1$ and also $4 \pi \epsilon_{0}=1$.

\subsection{Set-up: disformal transformation of a Kerr- Newman black hole}

We build a modification of Einstein gravity which includes additional vector degrees of freedom, belonging to the class of theories dubbed vector-tensor Galileons [10, ए75, ए76]. Such vector degrees of freedom can be associated with dark forces motivated by dark matter or dark energy model building [1.9:3]. Our aim will be to investigate new rotating black hole solutions for the theory we consider. In order to construct a modified gravity action, our starting point is a standard Einstein- 
Maxwell system, described by an action

$$
S_{E M}=\int d^{4} x \sqrt{-\tilde{g}}\left[\frac{\tilde{R}}{4}-\frac{1}{4} \tilde{F}^{\mu \nu} \tilde{F}_{\mu \nu}\right],
$$

where $\tilde{R}$ is the Ricci scalar and $\tilde{F}$ the electromagnetic field strength, and the corresponding Einstein's equation is

$$
\tilde{R}_{\mu \nu}-\frac{1}{2} \tilde{g}_{\mu \nu} \tilde{R}=\tilde{T}_{\mu \nu}
$$

with

$$
\tilde{T}_{\mu \nu}=\tilde{F}^{\mu \alpha} \tilde{F}_{\alpha}^{\nu}-\frac{1}{4} \tilde{g}^{\mu \nu} \tilde{F}_{\alpha \beta} \tilde{F}^{\alpha \beta}
$$

being the usual electromagnetic stress-energy tensor. Although we call the previous action an 'Einstein-Maxwell' system, as we are going to discuss the vector fields appearing in equation ([.]) should not be identified with standard electromagnetism, but with additional dark vector forces, namely hidden $U(1)$ gauge vectors related with dark energy and/or dark matter models. The theory enjoys an Abelian gauge invariance $A_{\mu} \rightarrow A_{\mu}+\partial_{\mu} \xi$ for any arbitrary function $\xi$, and it propagates four degrees of freedom, two in the transverse traceless tensor sector, and two in the transverse vector sector. Exact black hole solutions for the equations of motion associated for this theory are well studied, and include static Reissner-Nordström and rotating Kerr-Newman (KN) configurations, reviewed in chapter $\square$. As static/stationary solutions of Einstein-Maxwell theory, they satisfy no-hair theorems, and they are therefore uniquely defined in terms of their mass, angular momentum, and charge.

To generate new solutions, we act on this action with a disformal transformation [112, 13, 1194, 195] involving vector fields and parameterized by a real constant $\beta$, which plays a key role in what follows:

$$
\begin{aligned}
\tilde{g}_{\mu \nu}(x) & =g_{\mu \nu}(x)-\beta^{2} A_{\mu}(x) A_{\nu}(x), \\
\tilde{A}_{\mu}(x) & =A_{\mu}(x)+\partial_{\mu} \alpha(x),
\end{aligned}
$$


for any arbitrary function $\alpha(x)$ (the transformation of the vector simply reflects the gauge freedom of the original theory, and $\alpha$ does not appear in the final formulae). Firstly discussed in [12] as causal preserving transformations, disformal transformations have been largely studied in the last decade in the context of scalar-tensor theories of gravity, since they appear to transform such theories of gravity without adding any extra degree of freedom [1]:3]. In general, a disformal transformation of a metric $g_{\mu \nu}$ reads

$$
g_{\mu \nu} \rightarrow C\left(v_{\alpha} v^{\alpha}\right) g_{\mu \nu}+D\left(v_{\alpha} v^{\alpha}\right) v_{\mu} v_{\nu}
$$

with $v_{\mu}$ being the disformal vector and $C\left(v_{\alpha} v^{\alpha}\right)$ and $D\left(v_{\alpha} v^{\alpha}\right)$ the conformal and disformal coefficient, respectively. Although more general disformal/conformal transformations can be considered (see for example [ [194]), the transformation ([2.4) is sufficient for our purposes. The disformed metric is invertible, with inverse given by

$$
\tilde{g}^{\mu \nu}=g^{\mu \nu}+\beta^{2} \gamma_{0}^{2} A^{\mu} A^{\nu} \quad \text { with } \quad \gamma_{0}^{2}=\frac{1}{1-\beta^{2} A^{\mu} A_{\mu}} .
$$

Up to total derivatives, the disformed action reads

$$
S_{d i s f}=S_{E H}+S_{\text {matt }}=\int d^{4} x \sqrt{-g}\left(\mathcal{L}_{E H}+\mathcal{L}_{\text {matt }}\right)
$$

with Lagrangian densities

$$
\mathcal{L}_{E H}=\frac{1}{4 \gamma_{0}}\left[R-\frac{\beta^{2}}{4} \gamma_{0}^{2}\left(S_{\mu \nu} S^{\mu \nu}-S^{2}\right)+\frac{\beta^{2}}{4} F_{\mu \nu} F^{\mu \nu}+\frac{\beta^{4}}{2} \gamma_{0}^{2} F_{\mu \rho} F_{\nu}^{\rho} A^{\mu} A^{\nu}\right]
$$

and

$$
\mathcal{L}_{\text {matt }}=-\frac{1}{4 \gamma_{0}}\left[F^{\mu \nu} F_{\mu \nu}+2 \beta^{2} \gamma_{0}^{2} F_{\mu \rho} F_{\nu}^{\rho} A^{\mu} A^{\nu}\right],
$$

with

$$
\begin{aligned}
& F_{\mu \nu}=\nabla_{\mu} A_{\nu}-\nabla_{\nu} A_{\mu} \\
& S_{\mu \nu}=\nabla_{\mu} A_{\nu}+\nabla_{\nu} A_{\mu} \\
& S=S_{\mu \nu} g^{\mu \nu}
\end{aligned}
$$


In a compact notation, the full disformal Lagrangian reads:

$$
\mathcal{L}_{\text {disf }}=\frac{1}{4 \gamma_{0}}\left[R-\frac{\beta^{2}}{4} \gamma_{0}^{2}\left(S_{\mu \nu} S^{\mu \nu}-S^{2}\right)-\frac{4-\beta^{2}}{4} F_{\mu \nu} F^{\mu \nu}+\frac{\beta^{4}-4 \beta^{2}}{2} \gamma_{0}^{2} F_{\mu \rho} F_{\nu}^{\rho} A^{\mu} A^{\nu}\right] .
$$

The system after the disformal transformation describes a vector-tensor theory of gravity which deviates from the usual Einstein-Maxwell case ([.]) by quantities depending on the disformal parameter $\beta^{2}$. The action ( $[\mathbf{L} .2]$ ) will be our modified gravity theory of reference in the following. It contains non-minimal couplings of the vector to gravity, and derivative self-interactions of the form that usually characterize Horndeski systems. Moreover, the theory is free of Ostrogradsky's instabilities. In fact, this system belongs to the class of theories dubbed vectortensor Galileons or Generalised Proca, which have been investigated at length for their distinctive properties for cosmology [11, 1966-202], field theory [2013-213], and black holes [161, 177-184]. Disformal transformations are known to preserve the causality properties of the theory one starts with [12]: thus, since the EinsteinMaxwell theory is causally well behaved, one does not expect instabilities or causal pathologies in the theory described by equation ([2.T2).

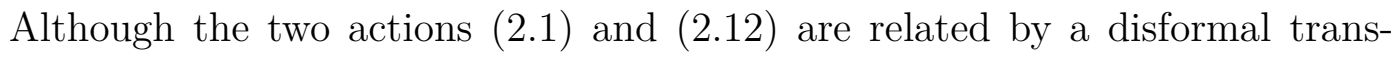
formation, they are not equivalent when additional matter, minimally coupled to gravity, is included into the system. Hence, interpreting the system of ( $\left.2 .{ }^{2}\right)$ as part of a more general action including matter fields, we can expect that its physical consequences can be different from an Einstein-Maxwell set-up (we will elaborate more on these points in the next sections). Action ([2.T2) breaks an Abelian gauge invariance, since the Lagrangian depends explicitly on the gauge potential $A_{\mu}$. On the other hand, it inherits some memory of the original gauge symmetry of action $(\underline{Z} . \mathbb{C})$; in fact, the final action is invariant, up to total derivatives, under the more general gauge transformation

$$
\begin{aligned}
g_{\mu \nu} & \rightarrow g_{\mu \nu}-\beta^{2} \partial_{\mu} \chi A_{\nu}-\beta^{2} \partial_{\nu} \chi A_{\mu}-\beta^{2} \partial_{\mu} \chi \partial_{\nu} \chi \\
A_{\mu} & \rightarrow A_{\mu}+\partial_{\mu} \chi
\end{aligned}
$$

with $\chi$ being an arbitrary scalar function. It is easy to check that the quanti- 


\section{A NEW ROTATING BLACK HOLE SOLUTION}

ties $\tilde{g}_{\mu \nu}$ and $\tilde{A}_{\mu}$ (equations ([2.4) and ([.2.5) ) are invariant under the simultaneous

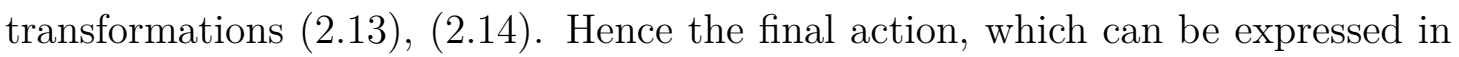
terms of the combinations $\tilde{g}_{\mu \nu}$ and $\tilde{A}_{\mu}$, is invariant under this symmetry. Notice that the limit $\beta \rightarrow 0$ reduces, as expected, to standard Abelian gauge symmetry. Such symmetry ensures that the final action propagates four dynamical modes. The absence of a fifth, scalar degree of freedom is a welcoming feature for phenomenology, since it can automatically avoid stringent constraints on the existence of long range scalar fifth forces. In our analysis, we focus on studying new regular rotating black holes for the theory ( $[. \cdot 2)$, equipped with the new gauge symmetry ([.13), ([.14), obtained by a disformal transformation of the Einstein-Maxwell action. Since the metric $\tilde{g}_{\mu \nu}$ is solution of the Einstein-Maxwell action ( $\mathbb{Z} \cdot \mathbf{D}$ ), we assume the following:

- in the static, spherically symmetric case, $\tilde{g}_{\mu \nu}$ is the the Reissner-Nordström metric $g^{R N}$ with corresponding gauge vector $A_{\mu}^{R N}$;

- in the stationary, axisymmetric case, $\tilde{g}_{\mu \nu}$ is the Kerr-Newman metric $g^{K N}$ with corresponding gauge vector $A_{\mu}^{K N}$.

Moreover, as we have already mentioned, the Einstein-Maxwell action ([2. D) does not simply describe electromagnetism, but it could be rather describe dark forces models. However, for simplicity and to not be pedantic, throughout the chapter we use the notation and terminology of the electromagnetic case, but the reader is aware that is just a convenience choice. Since the Kerr-Newman solution is the rotating generalisation of the static Reissner-Nordström one in the limit $a \rightarrow 0$, we now focus our attention on the more generic rotating case, being able to recover the static one when $a \rightarrow 0$. For an electrically charged rotating black hole, using a Boyer-Lindquist coordinate system the corresponding electromagnetic vector reads

$$
A_{\mu}^{K N}=\left\{A_{t}(r, \theta), 0,0, A_{\varphi}(r, \theta)\right\}
$$

with

$$
A_{t}(r, \theta)=\frac{-Q r}{r^{2}+a^{2} \cos ^{2} \theta}, \quad A_{\varphi}(r, \theta)=\frac{a Q \sin ^{2} \theta}{r^{2}+a^{2} \cos ^{2} \theta}
$$




\subsection{Set-up: disformal transformation of a Kerr-Newman black hole}

Again, the static Reissner-Nordström case is recovered setting $a=0$, and we get:

$$
A_{\mu}^{R N}=\left\{-\frac{Q}{r}, 0,0,0\right\}
$$

Both in the static and in the stationary case, the components $A_{r}$ and $A_{\theta}$ do not affect the geometry, and using the Abelian gauge symmetry and the equation of motion we can gauge them away and set them equal to zero. However, if we want to be completely generic (and this will be reveal itself useful in the following), the Kerr-Newman gauge vector is

$$
A_{\mu}^{K N}=\left\{A_{t}(r, \theta), A_{r}(r, \theta), A_{\theta}(r, \theta), A_{\varphi}(r, \theta)\right\}
$$

with $A_{t}, A_{\varphi}$ given by (2.16) and $A_{r}, A_{\theta}$ being the radial and the polar profiles, respectively. In particular, from the Einstein's equation ([2.2) we obtain the following condition on the radial and polar components:

$$
\tilde{F}_{r \theta}=\partial_{r} A_{\theta}(r, \theta)-\partial_{\theta} A_{r}(r, \theta)=0
$$

It is important to notice that the disformed action mixes metric and vector degrees of freedom, breaking Abelian gauge invariance. Therefore, it is not possible to simply gauge away the radial and polar profiles, and they consequently affect the geometry. So, the choice of both $A_{r}(r, \theta)$ and $A_{\theta}(r, \theta)$ is crucial for the resulting

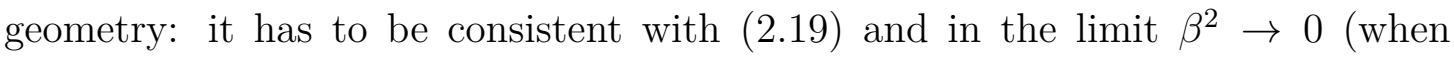
the disformal transformation is off and the action ([.]2) simply reduces to the

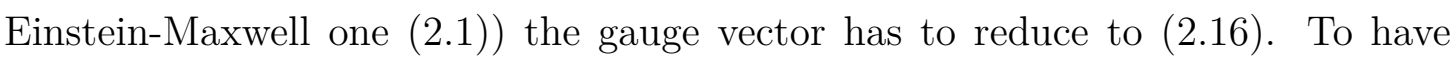
(Z.IG) satisfied, we have 4 different possibilities:

- both $A_{r}$ and $A_{\theta}$ vanish:

$$
\left\{A_{r}(r, \theta)=A_{\theta}(r, \theta)=0\right\}
$$

- $A_{\theta}$ vanishes, while $A_{r}$ is a function of the radial coordinate only (or viceversa):

$$
\left\{A_{r}(r), A_{\theta}(r, \theta)=0\right\} ;
$$


- $A_{\theta}$ is a function just of the angular variable $\theta$, while $A_{r}$ is a function of the radial coordinate only:

$$
\left\{A_{r}(r), A_{\theta}(\theta)\right\}
$$

- both $A_{r}$ and $A_{\theta}$ are generic functions of $r$ and $\theta$ :

$$
\left\{A_{r}(r, \theta), A_{\theta}(r, \theta)\right\}
$$

In the following sections, we study each case separately, since, as we will show, they lead to very different results.

\subsection{Singularities, Killing vectors and horizons}

Before moving to the study of the different scenarios corresponding to different choices of the gauge vector $A_{\mu}$, a very brief review on some basic geometrical objects is necessary for sections to come. For each of the different cases ( $2 \cdot 2 \pi, 2 \cdot 2]$, [2.22, [2.2.3), we have to check the regularity of the geometry and the position of the horizons if any singularity eventually appears. By definition, a singularity is a region of spacetime where the curvature tends to infinity. From this definition, since singularities are located at those points where the curvature invariants diverge, we have to be sure that eventual divergences of the curvature scalars are screened by an event horizon, avoiding the appearing of naked singularities.

To try to formally and intuitively define what an event horizon is, we first need some preliminary definitions. In General Relativity and more in general in pseudoRiemannian geometry, a null hypersurface is a hypersurface (namely a $(n-$ 1)-dimensional submanifold $\Sigma$ embedded in a $n$-dimensional manifold $\mathcal{M}$ ) whose normal vector is a null vector, and therefore it is both tangent and orthogonal to $\Sigma$. Specifying the hypersurface through a scalar function $f$ as

$$
f(x)=\text { const }
$$


the vector field

$$
\xi^{\mu}=g^{\mu \nu} \nabla_{\nu} f
$$

is manifestly normal to the hypersuface. Moreover, since we are considering a null hypersurface, on $\Sigma$ it is also true that

$$
\xi^{\mu} \xi_{\mu}=0
$$

which together with (2.25) leads to

$$
\xi^{\nu} \nabla_{\nu} \xi^{\mu}=0
$$

To be precise, the previous condition is satisfied if the geodesic corresponding to the tangent vector is affinely parametrised ${ }^{m}$ : otherwise, the geodesics equation reads

$$
\xi^{\mu} \nabla_{\mu} \xi_{\nu}=F(\tau) \xi_{\nu}
$$

where the scalar function $F$ is null if the parameter $\tau$ is the affine one: the affinely parametrised null geodesics $x^{\mu}(\tau)$ are known as the generators of the hypersurface $\Sigma$.

Instead, a Killing vector is an infinitesimal generator of an isometry on a manifold; given a metric $g$, a Killing vector $v^{\mu}$ represents a direction along which the metric is preserved, namely it corresponds to a quantity which is conserved along geodesics. Geometrically, the Lie derivative of the metric with respect to the Killing vector is null:

$$
\mathcal{L}_{v} g=0
$$

which in local coordinates can be written as

$$
\nabla_{(\mu} v_{\nu)}=0
$$

Therefore, a Killing horizon is defined as a null hypersurface on which the norm of a Killing vector vanishes. As already discussed in the previous chapter, we can naively but intuitively define an event horizon as a hypersurface separating those

\footnotetext{
${ }^{1}$ Indeed, the curves $x(\tau)$ such that $\xi^{\mu}=\frac{d x(\tau)}{d \tau}$ are actually geodesics.
} 


\section{A NEW ROTATING BLACK HOLE SOLUTION}

spacetime points which cannot affect any observer and event in the outside region. Equivalently, the event horizon can be intuitively defined as the boundary of the spacetime region from where light rays cannot totally escape. From the second definition, it is clear why the event horizon is a null hypersurface, and hence the relations ( $(2.2 .5)$ and $([2.27)$ must hold on the horizon.

For stationary, asymptotically flat spacetimes, there are convenient coordinate systems in which it is easy to find the event horizon position by observing the light cones "tilting over" as it happens in the Schwarzschild case [221, 25], in such a way that (inside the horizon) future oriented light rays propagate towards the singularity, with no chance to cross the horizon and escape. Since a stationary spacetime admits an asymptotically timelike Killing vector, on hypersurfaces $t=$ const we can choose a 3-dimensional coordinate system in which the metric at infinity is just the Minkowski one in a spherical coordinate system. So, at infinity, $r=$ const are timelike hypersurfaces; to find where they become null, it is sufficient to consider the normal vector to such hypersurfaces, namely:

$$
\partial_{\mu} r
$$

and to find where its norm becomes null:

$$
g^{\mu \nu} \partial_{\mu} r \partial_{\nu} r=g^{r r}=0 .
$$

Thus, the event horizon is defined by $r=r_{H}$ such that

$$
g^{r r}\left(r_{H}\right)=0
$$

For static black holes like Schwarzschild and Reissner-Nordström ones, the event horizon coincides with the Killing horizon associated with the time translation Killing vector

$$
k^{\mu}=\{1,0,0,0\}
$$

since it happens that

$$
\left.k^{\mu} k_{\mu}\right|_{r=r_{H}}=\left.\partial_{\mu} r \partial^{\mu} r\right|_{r=r_{H}}=0 .
$$


Instead, for stationary black holes like Kerr and Kerr-Newmann ones, the event horizon coincides with the Killling horizon associated with the Killing vector $\chi^{\mu}$ defined as

$$
\chi^{\mu}=k^{\mu}+\omega r^{\mu}
$$

with $k^{\mu}$ being the time translation Killing vector and $r^{\mu}$ the $\varphi$-rotation Killing vector

$$
r^{\mu}=\{0,0,0,1\}
$$

From the definition, we have

$$
\left.\chi^{\mu} \chi_{\mu}\right|_{r=r_{H}}=\left.\partial_{\mu} r \partial^{\mu} r\right|_{r=r_{H}}=0 .
$$

In general, for stationary geometries, the hypersurface where $k^{\mu} k_{\mu}=0$ is the ergosurface $^{\mathbb{m}}$, being the external boundary of the spacetime region (ergosphere) where no observer can remain stationary with respect to the black hole, since the spacetime is dragged by the black hole's rotation.

\subsection{New rotating solutions in vector-tensor the- ories of gravity}

In this section we show that a disformal transformation acting on an appropriate solution of the original field equations may lead to a new regular, rotating black hole solution for action ( $[.2 \mathrm{Z})$, with a non-trivial profile for the vector field. One might expect that the non-linearity of the field equations and the fact that we renounce spherical symmetry imply that rotating configurations in this theory are different from GR solutions. We confirm this expectation, showing that rotating configurations with non-trivial vector profiles have specific properties that make them distinguishable from their GR counterparts. This fact can lead to qualitatively new ways to test modified gravity models, by investigating the properties of

\footnotetext{
${ }^{1}$ Another common name in literature is infinite redshift surface, since the redshift of a stationary observer diverges on such hypersuface.
} 
their black hole solutions.

We start from a Kerr-Newman solution of the Einstein-Maxwell action ([ॄ.]), which can be expressed in Boyer-Lindquist coordinates as

$$
d s^{2}=\left(\frac{d r^{2}}{\Delta}+d \theta^{2}\right) \rho^{2}-\left(d t-a \sin ^{2} \theta d \varphi\right)^{2} \frac{\Delta}{\rho^{2}}+\left[\left(r^{2}+a^{2}\right) d \varphi-a d t\right]^{2} \frac{\sin ^{2} \theta}{\rho^{2}}
$$

together with the gauge vector

$$
A_{\mu}=\left\{-\frac{Q r}{\rho^{2}}, A_{r}(r, \theta), A_{\theta}(r, \theta), \frac{a Q r \sin ^{2} \theta}{\rho^{2}}\right\}
$$

where we have defined:

$$
\Delta=a^{2}+r^{2}-2 M r+Q^{2}, \quad \rho^{2}=r^{2}+a^{2} \cos ^{2} \theta
$$

As already discussed in chapter $\square$, the $\mathrm{KN}$ configuration describes a rotating charged black hole: see e.g. [201, 21] for comprehensive reviews on rotating black hole solutions in GR. The constants $M, Q$, and $a$ are associated with the black hole's mass, charge, and angular momentum, and they are the three Komar charges associated with Komar integrals of $k^{\mu}, r^{\mu}$ and $A^{\mu}$ : see the appendix [2.6 at the end of this chapter for more details. As we have already explained in the previous section, exploiting the Abelian gauge symmetry of the Einstein-Maxwell action we can set the radial and the polar components of the Kerr-Newman gauge vector to zero:

$$
A_{r}=A_{\theta}=0
$$

However, since the disformal transformation mixes metric and vector degrees of freedom, the standard Abelian symmetry is broken, and the radial and polar components of the gauge vector become crucial for the geometry. In the following, we will study 4 different choices for the radial and polar components, corresponding to the 4 cases ( $2.201,[2 \cdot 2],[2 \cdot 22,[2 \cdot 23)$. 


\subsubsection{Case 1: $A_{r}(r, \theta)=A_{\theta}(r, \theta)=0$}

We apply the disformal transformation of eq ([2.4) to equation (2.3. $)$, with disformal vector $A_{\mu}$ taking the form:

$$
A_{\mu}=\left\{-\frac{Q r}{\rho^{2}}, 0,0, \frac{a Q r \sin ^{2} \theta}{\rho^{2}}\right\} .
$$

The resulting configuration is a solution of the vector-tensor equations of motion

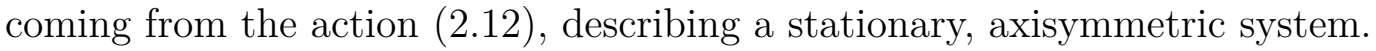

Using a Boyer-Lindquist coordinate system, the disformed metric reads

$$
d s^{2}=g_{t t} d t^{2}+g_{r r} d r^{2}+g_{\theta \theta} d \theta^{2}+g_{\varphi \varphi} d \varphi^{2}+2 g_{t \varphi} d t d \varphi
$$

with components

$$
\begin{aligned}
& g_{t t}=-1-\frac{\left(Q^{2}-2 M r\right)\left(r^{2}+a^{2} \cos ^{2} \theta\right)-\beta^{2} Q^{2} r^{2}}{\left(r^{2}+a^{2} \cos ^{2} \theta\right)^{2}} \\
& g_{t \varphi}=a \sin ^{2} \theta \frac{\left(Q^{2}-2 M r\right)\left(r^{2}+a^{2} \cos ^{2} \theta\right)-\beta^{2} Q^{2} r^{2}}{\left(r^{2}+a^{2} \cos ^{2} \theta\right)^{2}} \\
& g_{r r}=\frac{r^{2}+a^{2} \cos ^{2} \theta}{a^{2}+r^{2}-2 M r+Q^{2}}, \\
& g_{\theta \theta}=r^{2}+a^{2} \cos ^{2} \theta, \\
& g_{\varphi \varphi}=\sin ^{2} \theta\left\{r^{2}+a^{2}-\frac{a^{2} \sin ^{2} \theta\left[\left(Q^{2}-2 M r\right)\left(r^{2}+a^{2} \cos ^{2} \theta\right)-\beta^{2} Q^{2} r^{2}\right]}{\left(r^{2}+a^{2} \cos ^{2} \theta\right)^{2}}\right\} .
\end{aligned}
$$

Since the disformal vector ([.4.3) is exactly the Kerr-Newman gauge vector, the disformed metric has no additional components compared to the Kerr-Newman one. In particular, the metric is still stationary, axisymmetric and asymptotically flat, with only one non diagonal component $g_{t \varphi}$ corresponding with rotations around the symmetry axis. This geometry describes a rotating black hole with regular horizon, charged under the vector field $A_{\mu}$, where the dimensionless quantity $\beta^{2}$ parametrically controls deviations from the Kerr-Newman geometry in eq (2.68). Similarly to the Kerr-Newman solution, the geometry depends on three integra- 


\section{A NEW ROTATING BLACK HOLE SOLUTION}

tion constants $(M, a, Q)$ corresponding as usual at mass, spinning parameter and charge, respectively; moreover, the geometry also depends on the parameter $\beta^{2}$ associated the non-minimal couplings of vector to gravity in action ([2.Z2). Besides mass and spin, the geometry is charged under the vector degrees of freedom that control our modification of gravity (and should not be simply identified with electromagnetism). Using the Mathematica package $x A c t$ [214], we explicitly checked that equations $([2.68),([2.69)$ are a solution of all the equations of motion associated with the disformed vector-tensor action. It is important to emphasise that although this configuration is disformally related to Kerr-Newman, it is a new solution for the vector-tensor modified gravity theory we are considering.

\subsubsection{Horizons and singularities}

At this point, we have to study the position of the event horizon, the ergosurface and the spacetime singularity, to check whether the solution is everywhere regular outside the event horizon. As we will show soon, the event horizon is not affected by the disformal transformation, while both the ergosurface and the singularity depend on the parameter $\beta$, and therefore they are affected by the disformal transformation. Given a spherical coordinate system $\{t, r, \theta, \varphi\}$, the event horizon corresponds to points where the hypersurfaces of constant $r$ become null, given by the condition

$$
g^{r r}=0
$$

For the spacetime described by the metric ([2.44) with ([2.45), we find that the event horizon coincides with the Kerr-Newman one, namely

$$
r_{H}=M+\sqrt{M^{2}-a^{2}-Q^{2}} .
$$

This fact was quite expected, since the disformal transformation does not affect the radial components of the metric. However, since the disformal transformation affects the time component, it is not surprisingly that the ergosphere is different compared with the Kerr-Newman one. In fact, keeping in mind that the ergosurface is given by the condition

$$
k^{\mu} k_{\mu}=0
$$


with $k^{\mu}$ being the killing vector associated with stationarity ([2.34), the disformed ergosurface $\Sigma_{E}$ is defined by

$$
\Sigma_{E} \equiv\left(a^{2} \cos ^{2} \theta+r^{2}-2 M r+Q^{2}\right)\left(a^{2} \cos ^{2} \theta+r^{2}\right)-\beta^{2} Q^{2} r^{2}=0,
$$

to be compared with the Kerr-Newman one

$$
\Sigma_{E}^{K N} \equiv\left(a^{2} \cos ^{2} \theta+r^{2}-2 M r+Q^{2}\right)\left(a^{2} \cos ^{2} \theta+r^{2}\right)=0,
$$

which leads to

$$
r_{E}^{K N}=M+\sqrt{M^{2}-a^{2} \cos ^{2} \theta-Q^{2}}
$$

From ([2.49), it is clear that the Kerr-Newman case is recovered in the limit $\beta^{2} \rightarrow 0$, as expected. At this point, we have to compare the position of the event horizon with the ergosurface. As it can be seen from ([2.49), the ergosphere has the shape of an ellipsoid, with maximum radius on the equatorial plane $\left(\theta=\frac{\pi}{2}\right)$ :

$$
r_{E}^{\frac{\pi}{2}}=M^{2}+\sqrt{M^{2}-Q^{2}\left(1-\beta^{2}\right)}
$$

while at poles $(\theta=0, \pi)$ the radius takes its minimum value.

Assuming $\beta$ real and therefore $\beta^{2} \geq 0$, we have

$$
r_{E}^{\frac{\pi}{2}} \geq r_{E}^{0, \pi} \geq r_{H}
$$

since for a spinning black hole we have

$$
\beta^{2} Q^{2}>-a^{2}
$$

and therefore

$$
\Sigma_{E}\left(r_{H}\right)<0
$$

which prove both the inequalities in ([2.5.3); thus, the ergosurface is totally located outside the event horizon. At this point, we have to be sure that singularities are 
enclosed within the horizon. The expression for the Ricci scalar is

$$
R=\frac{\beta^{2} \zeta(r, \theta)}{\left(a^{2} \cos ^{2} \theta+r^{2}\right)^{4}\left[\left(a^{2}+r^{2}-2 M r+Q^{2}\right)\left(a^{2} \cos ^{2} \theta+r^{2}\right)-\beta^{2} Q^{2} r^{2}\right]^{2}},
$$

with $\zeta(r, \theta)$ being a everywhere regular function which does not affect the regularity of the geometry; thus, the Ricci scalar is divergent both on the Kerr ring

$$
r^{2}+a^{2} \cos ^{2} \theta=0
$$

and on the hypersurface $\Sigma_{S}$ such that

$$
\Sigma_{S} \equiv\left(a^{2}+r^{2}-2 M r+Q^{2}\right)\left(a^{2} \cos ^{2} \theta+r^{2}\right)-\beta^{2} Q^{2} r^{2}=0
$$

Therefore, in order to avoid naked singularities, we have to check whether the hypersurface $\Sigma_{S}$ is entirely inside the event horizon. Similarly to the ergosurface, $\Sigma_{S}$ is an ellipsoid with maximum radius on the equatorial plane, where it takes the value

$$
r_{S}^{\frac{\pi}{2}}=M+\sqrt{M^{2}-a^{2}-Q^{2}\left(1-\beta^{2}\right)}
$$

and so we have

$$
r_{S}^{\frac{\pi}{2}} \geq r_{E}^{\frac{\pi}{2}}
$$

where the equality is satisfied in the static limit $a=0$.

Moreover, from $(\overline{2.58})$ we observe that on the poles it happens

$$
\Sigma_{S}^{0, \pi}=\Sigma_{E}^{0, \pi}
$$

which shows that the hypersurface $\Sigma_{S}$ lies outside the ergosurface but they coincide at poles. Since we have already seen that the ergosurface is totally located outside the event horizon, with a little abuse of notation we can write

$$
\Sigma_{S} \geq \Sigma_{E}>r_{H}
$$

As one would expect, we recover the GR results in the limit $\beta \rightarrow 0$, when the geometry is everywhere outside the event horizon since the spacetime singularity 
appears at the Kerr ring

$$
r^{2}+a^{2} \cos ^{2} \theta
$$

completely inside the event horizon.

So, for $\beta \neq 0$ it is clear that the geometry admits a naked singularity, with no horizon to screen it. From these considerations, the geometry (2.44) with (2.45) cannot be considered a regular black hole, and so the choice $A_{r}=A_{\theta}=0$ for the disformal transformation ([2.4) does not lead to a physical solution.

\subsubsection{Case 2: $A_{r}(r), A_{\theta}(r, \theta)=0$}

As second case, we apply the disformal transformation ([2.4) to ([2.3.4), assuming a disformal vector with radial and polar components such that:.

$$
A_{r}(r), \quad A_{\theta}=0
$$

The resulting configuration is a axisymmetric, stationary solution of the vectortensor theory with action ( $(2 . T 2)$. As we have seen in the previous case, the specific shape of the disformal vector affects the geometry; in this case, requiring regularity on both the horizon and the ergosurface, we found the following specific profile for the radial vector component $A_{r}$ which leads to a regular, asymptotically flat black hole configuration:

$$
A_{r}(r)=\frac{Q r}{\Delta}
$$

It would be interesting to understand whether other radial vector profiles give regular solutions, although it seems that (2.6.5) is the only one which ensures both a disformal horizon and regularity everywhere outside it. With this choice for the radial vector component, the disformed metric contains off-diagonal components $d r d t$ and $d r d \varphi$, and the final metric reads

$$
d s^{2}=g_{t t} d t^{2}+g_{r r} d r^{2}+g_{\theta \theta} d \theta^{2}+g_{\varphi \varphi} d \varphi^{2}+2 g_{t r} d t d r+2 g_{t \varphi} d t d \varphi+2 g_{r \varphi} d r d \varphi
$$


with

$$
\begin{aligned}
g_{t t} & =-1-\frac{\left(Q^{2}-2 M r\right)\left(r^{2}+a^{2} \cos ^{2} \theta\right)-\beta^{2} Q^{2} r^{2}}{\left(r^{2}+a^{2} \cos ^{2} \theta\right)^{2}}, \\
g_{t r} & =\frac{-\beta^{2} Q^{2} r^{2}}{\left(a^{2}+r^{2}-2 M r+Q^{2}\right)\left(r^{2}+a^{2} \cos ^{2} \theta\right)}, \\
g_{t \varphi} & =a \sin ^{2} \theta \frac{\left(Q^{2}-2 M r\right)\left(r^{2}+a^{2} \cos ^{2} \theta\right)-\beta^{2} Q^{2} r^{2}}{\left(r^{2}+a^{2} \cos ^{2} \theta\right)^{2}}, \\
g_{r r} & =\frac{\left(a^{2}+r^{2}-2 M r+Q^{2}\right)\left(r^{2}+a^{2} \cos \theta^{2}\right)+\beta^{2} Q^{2} r^{2}}{\left(a^{2}+r^{2}-2 M r+Q^{2}\right)^{2}}, \\
g_{r \varphi} & =\frac{\beta^{2} a Q^{2} r^{2} \sin ^{2} \theta}{\left(a^{2}+r^{2}-2 M r+Q^{2}\right)\left(r^{2}+a^{2} \cos ^{2} \theta\right)}, \\
g_{\theta \theta} & =r^{2}+a^{2} \cos ^{2} \theta, \\
g_{\varphi \varphi} & =\sin ^{2} \theta\left\{r^{2}+a^{2}-\frac{a^{2} \sin ^{2} \theta\left[\left(Q^{2}-2 M r\right)\left(r^{2}+a^{2} \cos ^{2} \theta\right)-\beta^{2} Q^{2} r^{2}\right]}{\left(r^{2}+a^{2} \cos ^{2} \theta\right)^{2}}\right\} .
\end{aligned}
$$

In a more compact notation, we can write

$$
\begin{aligned}
d s^{2}= & {\left[-1-\frac{\left(Q^{2}-2 M r\right) \rho^{2}-\beta^{2} Q^{2} r^{2}}{\rho^{4}}\right] d t^{2}+\frac{\Delta \rho^{2}+\beta^{2} Q^{2} r^{2}}{\Delta^{2}} d r^{2}+\rho^{2} d \theta^{2} } \\
& +\sin ^{2} \theta\left[r^{2}+a^{2}-\frac{a^{2} \sin ^{2} \theta\left[\left(Q^{2}-2 M r\right) \rho^{2}-\beta^{2} Q^{2} r^{2}\right]}{\rho^{4}}\right] d \varphi^{2} \\
& -\frac{\beta^{2} Q^{2} r^{2}}{\Delta \rho^{2}} d t d r+a \sin ^{2} \theta \frac{\left(Q^{2}-2 M r\right) \rho^{2}-\beta^{2} Q^{2} r^{2}}{\rho^{4}} d t d \varphi-\frac{\beta^{2} a \sin ^{2} \theta Q^{2} r^{2}}{\Delta \rho^{2}} d r d \varphi
\end{aligned}
$$

with $\Delta, \rho^{2}$ given as in (‥4]) and the gauge field being

$$
A_{\mu}=\left(A_{t}, A_{r}, A_{\theta}, A_{\varphi}\right)=\left(-\frac{Q r}{\rho^{2}}, \frac{Q r}{\Delta}, 0, \frac{Q r a \sin ^{2} \theta}{\rho^{2}}\right) .
$$

This geometry describes a rotating black hole with regular horizon, charged under the vector field $A^{\mu}$ : 
- the dimensionless quantity $\beta^{2}$ parametrically controls deviations from the Kerr-Newman geometry in equation ([2.68). Again, the geometry depends on three integration constants, $M, a$, and $Q$ (see the appendix [2.6]), and on the parameter $\beta^{2}$. As we will see in more detail in the next sections, the contributions coming from $\beta$ modify the structure of the black hole horizons, also affecting the geodesics of particles moving close to the black hole;

- the vector field profile ([2.6.9) has three physical components turned on, against the two of the Kerr-Newman configuration. In this case, the radial component of the gauge vector is physical, and it cannot be gauged away without simultaneously changing the geometrical properties of the system.

Again, we emphasise that although this configuration is disformally related to Kerr-Newman, it is a new solution for the vector-tensor modified gravity theory we are considering; in fact, the systems $([]$.$) and \left(\left[\begin{array}{l}2.2 \\ 2.2\end{array}\right)\right.$ can have distinct physical properties when additional matter coupled with gravity is included (as an example, in section [2.4.2.3] we will show how time-like geodesics around such "disformal black hole" are different with respect to rotating black holes in GR).

An important, distinctive difference from the Kerr-Newman GR solution are the curvature invariants. The Ricci scalar associated with the geometry described by $(2.68)$ and $(2.69])$ is

$$
R=2 \beta^{2} a^{2} Q^{2} \cos ^{2} \theta \frac{\left(3 r^{2}-a^{2} \cos ^{2} \theta\right)}{\left(r^{2}+a^{2} \cos ^{2} \theta\right)^{4}}
$$

since the Ricci scalar is non-vanishing, this configuration is different from the original KN solution, where this quantity is equal to zero. However, similarly to GR, the unique geometrical singularity associated with our new disformed solution (ए.68) is the Kerr singularity at the locus

$$
r^{2}+a^{2} \cos ^{2} \theta=0
$$

since all the curvature invariants are everywhere regular except for the ring located at ([.]T). Since at asymptotic infinity the metric components approach flat space 
and the curvature invariants asymptotically tend to zero, the disformal solution is asymptotically flat. Before discussing in some detail the non-linear features of this disformed configuration, it is interesting to analyse the limit of small rotation: at first order in an expansion on the rotation parameter $a$, the geometry reads

$$
\begin{aligned}
d s^{2} & =\left(-1+\frac{2 M}{r}-\frac{\left(1-\beta^{2}\right) Q^{2}}{r^{2}}\right) d t^{2}+\frac{r^{4}}{\Delta^{2}}\left(1-\frac{2 M}{r}+\frac{Q^{2}\left(1+\beta^{2}\right)}{r^{2}}\right) d r^{2}+r^{2} \sin ^{2} \theta d \varphi^{2} \\
& +r^{2} d \theta^{2}-\frac{2 \beta^{2} Q^{2}}{\Delta} d t d r+2 a \sin ^{2} \theta\left[-\frac{2 M}{r}+\frac{Q^{2}\left(1-\beta^{2}\right)}{r^{2}}\right] d t d \varphi+\frac{2 \beta^{2} a Q^{2} \sin ^{2} \theta}{\Delta} d r d \varphi
\end{aligned}
$$

where $\Delta$ is obtained from $\left(\underline{Z .4}\right.$ ) with $a^{2}=0$, and in the static limit $a \rightarrow 0$ the metric becomes

$$
\begin{aligned}
d s^{2}= & \left(-1+\frac{2 M}{r}-\frac{\left(1-\beta^{2}\right) Q^{2}}{r^{2}}\right) d t^{2}+\frac{r^{4}}{\Delta^{2}}\left(1-\frac{2 M}{r}+\frac{Q^{2}\left(1+\beta^{2}\right)}{r^{2}}\right) d r^{2} \\
& +r^{2} d \theta^{2}+r^{2} \sin ^{2} \theta d \varphi^{2}-\frac{2 \beta^{2} Q^{2}}{\Delta} d t d r
\end{aligned}
$$

which can be considered the disformal Reissner-Nordström solution.

\subsubsection{Structure and properties of horizons}

In this section we study the horizons structure, emphasizing deviations from the

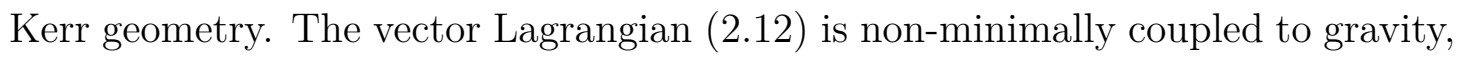
and the non-linear derivative interactions induce qualitative deviations from GR rotating solutions (for example, we will learn that our black holes can rotate faster than their GR counterparts, for the same mass and charge). Given a spherical coordinate system $\{t, r, \theta, \varphi\}$, the event horizon corresponds to points where the hypersurfaces of constant $r$ become null, namely

$$
g^{r r}=0
$$

For stationary geometries as our configuration, the ergosphere corresponds to the Killing horizon of the time translation Killing vector $k^{\mu}=\{1,0,0,0\}$. A priori, ergosphere and event horizon are distinct hypersurfaces: see [2:3] for details. For 
the standard $\mathrm{KN}$ black hole (obtained from solution (‥68) setting $\beta^{2}=0$ ), the exterior event horizon and the ergosphere are respectively located at

$$
\begin{array}{r}
r_{h o r}^{K N}=M+\sqrt{M^{2}-a^{2}-Q^{2}}, \\
r_{e r g}^{K N}=M+\sqrt{M^{2}-a^{2} \cos ^{2} \theta-Q^{2}} .
\end{array}
$$

Using Boyer-Lindquist coordinates, the Kerr event horizon is spherical, being located to a radial position independent on the angular coordinates (although the intrinsic horizon geometry is actually squashed, as manifest using appropriate coordinate systems [2:3]). Instead, the Kerr ergosphere is an ellipsoid, as it can be noticed from the angular dependence of the "radius" $r_{\text {erg }}^{K N}$. In the vector-tensor theory of gravity we are studying, the event horizon and the ergospheres are hypersurfaces of revolution around the azymuthal coordinate. Their positions is given by the real positive solutions of equations ${ }^{\text {m }}$

Hor: $g^{r r}=0 \Rightarrow f_{h} \equiv\left(r^{2}+a^{2} \cos ^{2} \theta\right)\left(r^{2}-2 M r+a^{2}+Q^{2}\right)-\beta^{2} Q^{2} r^{2}=0$,

Erg: $\quad k^{\mu} k_{\mu}=0 \Rightarrow f_{e} \equiv\left(r^{2}+a^{2} \cos ^{2} \theta\right)\left(r^{2}-2 M r+a^{2} \cos ^{2} \theta+Q^{2}\right)-\beta^{2} Q^{2} r^{2}=0$.

Equations $f_{h, e}=0$ are algebraic equations of fourth order in the coordinate $r$ : they can be solved analytically, but their solutions are complicated. Depending on the sign of the discriminant, they can have four, two or no real roots; we denote their maximal real roots with $r_{h}$ and $r_{e}$ respectively, corresponding to the horizon and the ergosurface position, respectively. The external horizon of the black hole is located at $r_{h}(r, \theta)$; since such expression depends on the polar angle $\theta$, in this configuration neither the ergosurface nor the horizons have spherical shape in Boyer-Lindquist coordinates. This is a major qualitative difference with respect to Kerr-Newman black holes, which have spherical horizons in Boyer-Lindquist

\footnotetext{
${ }^{1}$ We are aware that, in order to have a null hypersurface and hence a horizon, the null norm condition is not sufficient, since tangency condition ( $[2.27)$ is also required. In our case, the tangency condition is not satisfied on the horizon. For this reason, technically it is not an absolute horizon, but it could be rather considered an apparent one. However, with a bit of abuse of language we will consider and refer to this hypersurface as a proper horizon, in order to see if there is any interesting and peculiar feature arising from this geometry.
} 
coordinates. For consistency, we checked that substituting the value $r_{e}$ in the expression for $f_{h}$ ( $\left.2 \cdot 76\right)$ we get $f_{h} \geq 0$, hence the position of the ergosurface is outside the horizon: $r_{e} \geq r_{h}$. The equality is satisfied at poles $(\theta=0, \pi)$, where the horizon and the ergosurface coincide: $f_{e}=f_{h}$. The only value of the polar angle where an expression for $r_{h, e}$ can be analytically obtained is on the equatorial plane $(\theta=\pi / 2)$, where we get

$$
\begin{aligned}
& r_{h}^{\pi / 2}=M+\sqrt{M^{2}-a^{2}-Q^{2}\left(1-\beta^{2}\right)}, \\
& r_{e}^{\pi / 2}=M+\sqrt{M^{2}-Q^{2}\left(1-\beta^{2}\right)} .
\end{aligned}
$$

It is simple to show that the external horizon of Kerr-Newman black hole is always in the interior of the horizon of our disformal black hole configuration, for the same values of mass, charge and angular momentum. Substituting the value $r_{h}^{K N}$ for the position of the Kerr-Newman horizon in the expression ([2.76]) for $f_{h}$, we find that this quantity is negative, hence it lies inside the disformal black hole horizon. Figure $[$.$] in the next page shows the shape of black hole horizons and ergosphere$ for our systems, using Boyer-Lindquist coordinates.

At this point it is interesting to look at the event horizon's angular velocity. Let's consider a photon emitted along the $\varphi$ direction at radial distance $r$; thus, there are no components of the velocity along the $\theta$ and $r$ directions, and the metric simply reads

$$
d s^{2}=g_{t t} d t^{2}+2 g_{t \varphi} d t d \varphi+g_{\varphi \varphi} d \varphi^{2}=0
$$

which can be solved to obtain

$$
\frac{d \varphi}{d t}=-\frac{g_{t \varphi}}{g_{\varphi \varphi}} \pm \sqrt{\left(\frac{g_{t \varphi}}{g_{\varphi \varphi}}\right)^{2}-\frac{g_{t t}}{g_{\varphi \varphi}}} .
$$

Evaluating this expression on the event horizon

$$
f_{h}=0
$$


the term in the square root term vanishes and we are left with

$$
\left.\frac{d \varphi}{d t}\right|_{r_{h}}=\Omega_{h}=\frac{a}{a^{2}+r_{h}^{2}}
$$

which is the angular velocity of the event horizon. Notice that since the position of the horizon depends on the angular coordinate $\theta$, the angular velocity is not constant on the horizon.

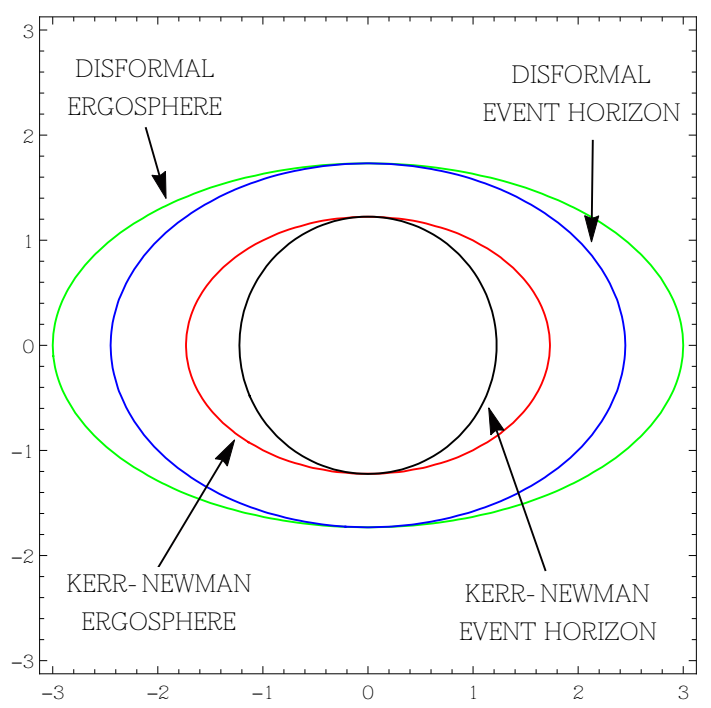

Figure 2.1: Pictorial representation of the shape of horizon and ergosphere for the disformal black hole using Boyer-Lindquist coordinates, as discussed in the main text.

\subsubsection{Maximal black hole spin and horizon oblateness}

The modifications of the geometry proportional to the parameter $\beta$ can deform the horizon, making it oblate also in Boyer-Lindquist coordinates. Interestingly, they can also allow for ultra spinning black holes, i.e. black hole configurations that rotate faster than Kerr. It is not easy to analytically study these properties of the geometry, except two special cases that we are going to investigate in this subsection. 


\section{A NEW ROTATING BLACK HOLE SOLUTION}

\section{The limit of large value of $\beta^{2}$}

The size of the disformal horizon increases with the polar angular coordinate: its smallest value is at the poles $(\theta=0, \pi)$, while its maximal value is on the equatorial plane $(\theta=\pi / 2)$. The existence of a regular horizon depends on the value of the black hole spin parameter $a$ : beyond an extremal value $a_{\max }$, an horizon ceases to exist. Such value of $a_{\max }$ can be found by studying the equation which gives the radial position of the horizon at the poles in Boyer-Lindquist coordinates. We focus on the poles because at this position we get the most stringent condition on $a_{\max }$. The radial horizon equation at the poles can be expressed as

$$
r^{4}-2 M r^{3}+\left(2 a^{2}+q M^{2}\right) r^{2}-2 a^{2} M r+a^{4}+\frac{a^{2} M^{2} q}{1-\beta^{2}}=0,
$$

where we define the combination

$$
q \equiv \frac{Q^{2}}{M^{2}}\left(1-\beta^{2}\right)
$$

which can be positive or negative, depending on the size of $\beta$. Analytic solutions for equation $([2.83])$ are cumbersome, but a great simplification occurs in the limit $|\beta| \gg$ 1. So, we study this regime maintaining a fixed value for the parameter $q$ defined in equation (2.84). This implies that we simultaneously take a limit in which black hole charge $Q$ becomes smaller and smaller, so to maintain $q$ finite. Such limit physically corresponds to a regime of strong non-minimal coupling between vector

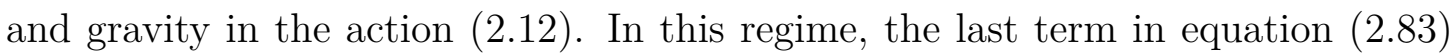
can be neglected, and the equation admits a simple expression for its real roots: the external horizon is located at

$$
r_{h}^{p o l}=\frac{M}{2}\left(1+\sqrt{1-q}+\sqrt{2-q+2 \sqrt{1-q}-4 \frac{a^{2}}{M^{2}}}\right) .
$$

Requiring a positive argument for the last square root in the previous expression gives us an upper bound on the rotation parameter $a$, and we find that its largest 
allowed value is given by

$$
\begin{aligned}
a_{\max } & =\frac{M}{2} \sqrt{2-q+2 \sqrt{1-q}} \\
& =\frac{M}{2} \sqrt{2+\frac{Q^{2}}{M^{2}}\left(\beta^{2}-1\right)+2 \sqrt{1+\frac{Q^{2}}{M^{2}}\left(\beta^{2}-1\right)}},
\end{aligned}
$$

where in the second line we used the definition ([2.84).

When $\beta^{2}>1$, then $a_{\max } \geq M$ (with equality saturated for $Q=0$ ), with $M$ being the maximal value of the spin $a$ for a Kerr black hole (while for Kerr-Newman the maximal spin is $\left.\sqrt{M^{2}-Q^{2}}<M\right)$. Thus, when $\beta^{2}$ is large our disformed rotating black hole can have angular momentum parametrically larger than in Einstein gravity (ultraspinning black holes are however possible in theories with more than $3+1$ dimensions [215] or in modified gravity theories including a complex scalar [157] or EdGB [188]). We obtained this result in the extremal limit of very large $|\beta|$ : it is possible to check numerically that for smaller values of $|\beta|$, the value of $a_{\max }$ reduces its size with respect to ([2.87), by a quantity that is proportional to $1 /|\beta|$. In any case, we will use $a_{\max }$ of $([2.87)$ as reference for our discussion. We also checked that the conditions on the parameter $a$ for having a regular horizon are most stringent at the poles, and less restrictive at other angular positions. In other words, the horizon at the equator could in principle rotate faster than $a_{\max }$ of $([2.86])$, but the requirement of having a regular horizon everywhere does not allow for this.

When the spin parameter attains the value $a_{\max }$, the ratio between the radial size of the horizon at the poles versus the size of the horizon at the equator quantifies the oblateness $\omega$ of the black hole in Boyer-Lindquist coordinates:

$$
\omega=1-\frac{r_{h}^{p o l}}{r_{h}^{e q}}=1-\frac{1+\sqrt{1-q}}{2+\sqrt{2-3 q-2 \sqrt{1-q}}}
$$

In the limit of large, negative values of $q$, oblateness approaches the extremal value $\omega_{\max }=1-1 / \sqrt{3}$ : hence in this limit the radial size of the horizon at the poles is $1 / \sqrt{3} \simeq 0.57$ times smaller than the size of the horizon at the equator, as measured in Boyer-Lindquist coordinates. 


\section{A NEW ROTATING BLACK HOLE SOLUTION}

We can interpret these horizon properties we determined as due to our non minimal vector-tensor interactions, which are able to contrast strong centrifugal forces, and maintain a regular horizon even for large spins, at the price of deforming the horizon shape. As we will discuss in more details in the next sections, the shape of the event horizon is very important, since it could be an observable and therefore distinctive feature of some astrophysical black hole.

\section{Massless black holes}

Interestingly, if $\beta^{2}>1$, our black hole configuration (2.68) admits a regular horizon even in the massless limit $M \rightarrow 0$. In this limit, the solution has two horizons: the radial position of the external one depends on the polar angle and is given by the following expression (valid if $\beta^{2}>1$ ):

$$
\begin{aligned}
r_{h}^{2}= & \frac{1}{2}\left[Q^{2}\left(\beta^{2}-1\right)-a^{2}\left(1+\cos ^{2} \theta\right)\right. \\
& +\sqrt{\left.\left[Q^{2}\left(\beta^{2}-1\right)-a^{2}\left(1+\cos ^{2} \theta\right)\right]^{2}-4 a^{2}\left(a^{2}+Q^{2}\right) \cos ^{2} \theta\right]} .
\end{aligned}
$$

The radial size of the black hole is maximal at the equator $(\theta=\pi / 2)$ and minimal at the poles $(\theta=0, \pi)$. The condition of having a regular horizon at the poles imposes an upper bound on the black hole's spin parameter, given by

$$
a_{\max }^{2}=Q^{2} \frac{\left(\beta^{2}-1\right)^{2}}{4 \beta^{2}}
$$

It is also simple to compute the black hole's oblateness for extremal values of the black hole's spin, as done in the previous paragraph around equation ([2.88). In this case we obtain

$$
\omega=1-\sqrt{\frac{1+\beta^{2}}{1+3 \beta^{2}}} .
$$

Similarly to the previous case (large $\beta$ limit), the maximal oblateness is still $1-$ $1 / \sqrt{3}$, showing that for large $\beta$ the radial size of the horizon at the poles is $1 / \sqrt{3} \simeq$ 0.57 times smaller than the size of the horizon at the equator. 


\subsubsection{Equatorial orbits}

The dynamics of massive and massless fields orbiting around rotating black holes is a broad subject with several ramifications and applications to astrophysics and cosmology, and it is the first step towards the study of black hole accretion disks, or of the extraction of rotational energy from spinning black holes: see [20] for an enlightening review, and [216] for the original paper studying this family of orbits in Kerr configurations. Here we focus our attention to circular orbits for massive particles in the equatorial plane, examining features that are peculiar of the disformed rotating black hole ([2.68). We make the hypothesis that the particles are only minimally coupled to gravity. Since the geometry is axially symmetric, stable orbits exist which remain confined on the equatorial plane. Having an exact form for the metric allows us to point out distinctive properties of equatorial orbits by simple, analytical considerations, which are a natural generalization of arguments developed for the Kerr/Newman geometry [216].

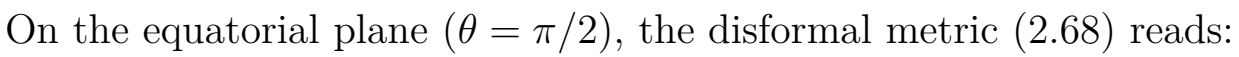

$$
d s^{2}=g_{t t} d t^{2}+g_{r r} d r^{2}+g_{\theta \theta} d \theta^{2}+g_{\varphi \varphi} d \varphi^{2}+2 g_{t r} d t d r+2 g_{t \varphi} d t d \varphi+2 g_{r \varphi} d r d \varphi
$$

with components

$$
\begin{aligned}
& g_{t t}=-\frac{r(r-2 M)+\left(1-\beta^{2}\right) Q^{2}}{r^{2}}, \\
& g_{r r}=\frac{r^{2}\left[a^{2}+r(r-2 M)+\left(1+\beta^{2}\right) Q^{2}\right]}{\Delta^{2}}, \\
& g_{\theta \theta}=r^{2}, \\
& g_{\varphi \varphi}=r^{2}+\frac{a^{2}\left[r(2 M+r)-\left(1-\beta^{2}\right) Q^{2}\right]}{r^{2}}, \\
& g_{t r}=-\frac{\beta^{2} Q^{2}}{\Delta}, \\
& g_{t \varphi}=\frac{a\left[\left(1-\beta^{2}\right) Q^{2}-2 M r\right]}{r^{2}}, \\
& g_{r \varphi}=\frac{\beta^{2} a Q^{2}}{\Delta} .
\end{aligned}
$$


Such metric is independent from $t$ and $\varphi$, hence we can define the conserved energy per unit mass $E$ and the conserved angular momentum per unit mass $L$ along the symmetry axis:

$$
\begin{aligned}
& E \equiv-k^{\mu} u^{\nu} g_{\mu \nu}, \\
& L \equiv r^{\mu} u^{\nu} g_{\mu \nu},
\end{aligned}
$$

where $u^{\mu}$ is the 4 -velocity vector, and $k^{\mu}$ and $r^{\mu}$ are the Killing vectors associated with time translations and $\varphi$-rotations, as defined in ([.34) and ([.37), respectively. Using the metric ([.92), the previous equations can be expressed in the following way:

$$
\begin{aligned}
& E=-\left(g_{t t} u^{t}+g_{t \varphi} u^{\varphi}\right), \\
& L=g_{\varphi t} u^{t}+g_{\varphi \varphi} u^{\varphi}
\end{aligned}
$$

with ( $\tau$ being proper time)

$$
u^{\mu}=\left\{u^{t}, u^{r}, u^{\theta}, u^{\varphi}\right\}=\left\{\frac{d t}{d \tau}, \frac{d r}{d \tau}, \frac{d \theta}{d \tau}, \frac{d \varphi}{d \tau}\right\} .
$$

Inverting the previous relations, one obtains the angular velocity at fixed radial distance from the equator,

$$
\frac{u^{\varphi}}{u^{t}}=\Omega=\frac{L\left[r(r-2 M)+\left(1-\beta^{2}\right) Q^{2}\right]-a E\left[\left(1-\beta^{2}\right) Q^{2}-2 M r\right]}{E r^{4}+a^{2} E\left[r(2 M+r)-\left(1-\beta^{2}\right) Q^{2}\right]+a L\left[\left(1-\beta^{2}\right) Q^{2}-2 M r\right]} .
$$

Time-like geodesics associated with massive particles satisfy the condition

$$
u^{\mu} u^{\nu} g_{\mu \nu}=-1
$$

To compute the radial position of stable, circular time-like trajectories on the equatorial plane, we assume that $u^{\theta}=0$, and we can combine equations ([.9.5) and ([..98) to obtain the following expression for derivatives of the radial position of a massive particle along the proper time:

$$
\begin{gathered}
\frac{r^{4}}{\Delta^{2}}\left[\Delta^{2}-\beta^{4} Q^{4}\right]\left(\frac{d r}{d \tau}\right)^{2}-\frac{2 \beta^{2} r^{2} Q^{2}}{\Delta}\left(a^{2} E-a L+E r^{2}\right)\left(\frac{d r}{d \tau}\right) \\
-\left(a^{2} E-a L+E r^{2}\right)^{2}+\left(\Delta-\beta^{2} Q^{2}\right)\left(1+\frac{(a E-L)^{2}}{r^{2}}\right)=0 .
\end{gathered}
$$


The previous equation can be recast as

$$
\frac{\left[\Delta^{2}-\beta^{4} Q^{4}\right]}{2 \Delta^{2}}\left(\frac{d r}{d \tau}\right)^{2}-\frac{\beta^{2} Q^{2}}{r^{2} \Delta}\left(a^{2} E-a L+E r^{2}\right)\left(\frac{d r}{d \tau}\right)+V_{e f f(r, E, L)}=\frac{E^{2}-1}{2}
$$

where we have define an effective potential

$$
V_{e f f}=-\frac{M}{r}+\frac{a^{2}\left(1-E^{2}\right)+L^{2}+\left(1-\beta^{2}\right) Q^{2}}{2 r^{2}}-\frac{M(L-a E)^{2}}{r^{3}}+\frac{\left(1-\beta^{2}\right) Q^{2}(L-a E)^{2}}{2 r^{4}} .
$$

The effective potential above depends on both energy and angular momentum.

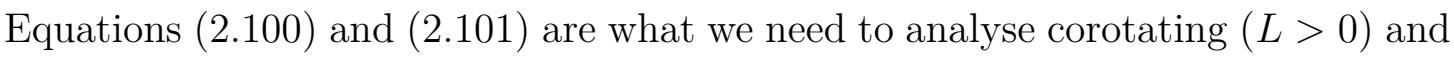
counterrotating $(L<0)$ circular trajectories, associated with objects having angular momentum parallel or antiparallel to the black hole's one. Before continuing, it is important to notice that the expression for the potential ([.]U⿴囗十) has a structure identical to the $\mathrm{KN}$ case, which is recovered in the limit $\beta^{2} \rightarrow 0$. Interestingly, new opportunities arise for our case with non-linear vector-tensor interactions. In the regime $\beta^{2}>1$, the relative sign among different contributions to the effective potential ( 2.100 ) changes with respect to standard KN black holes (in that case, this regime would correspond to an unphysical negative square charge). Hence this regime is interesting since it can lead to qualitatively new features for circular orbits. As a concrete example, a straightforward computation starting from equation ([2.97) leads to the following expression for the angular velocity of massive particles on circular orbits

$$
\Omega=\frac{M}{a M \pm \frac{r^{2} M}{\sqrt{r M+Q^{2}\left(\beta^{2}-1\right)}}},
$$

with \pm indicating orbits corotating/counterrotating with the black hole. If $\beta^{2}>1$, corotating orbits spin faster than the corresponding Kerr-Newman case, for identical values of the asymptotic charges $(M, Q, a)$.

We now proceed examining the existence and properties of marginally stable innermost circular orbits for test particles moving on the equatorial plane of our 
geometry, lying on a fixed radial position $\bar{r}$ in Boyer-Lindquist coordinates. Such trajectories are commonly referred to as simply ISCOs (Innermost Stable Circular Orbits) [2:3]. To move on a circular orbit of radius $\bar{r}$, both the initial radial velocity and the radial acceleration must vanish, and hence we have $\mathrm{t}$ impose:

$$
\begin{aligned}
& V_{e f f}(\bar{r}, E, L)=\frac{E^{2}-1}{2}, \\
& \left.\frac{\partial V_{e f f}(r, E, L)}{\partial r}\right|_{r=\bar{r}}=0 .
\end{aligned}
$$

Furthermore, the condition of stability implies that the particle must be at a minimum point of the potential, namely

$$
\left.\frac{\partial^{2} V_{e f f}(r, E, L)}{\partial r^{2}}\right|_{r=\bar{r}} \geq 0,
$$

where equality holds for the marginally stable circular trajectories that we are investigating. The previous three conditions lead to three equations which determine the three quantities $(E, L, \bar{r})$ characterizing marginally stable innermost trajectories. In order to express formulae in the simplest possible way, it is convenient to rescale our quantities as follows:

$$
x=\frac{\bar{r}}{M}, \quad \hat{a}=\frac{a}{M}, \quad q=\frac{Q^{2}}{M^{2}}\left(1-\beta^{2}\right) .
$$

Notice that $q$ can be negative, if $\beta^{2}>1$ : a negative $q$ can not be obtained in a Kerr-Newman-like configuration, and is distinctive of our disformed black hole geometry. We can now re-express and solve the three equations ([2.103])-([2.104) (with inequality saturated) in terms of the three unknown quantities $(E, L, x)$. After various algebraic manipulations, we get the following expressions for $E$ and $L$ in terms of $x$ :

$$
\begin{aligned}
E^{2} & =\frac{q(3-4 x)+x(3 x-2)}{x(3 x-4 q)} \\
\frac{L^{2}}{M^{2}} & =\frac{\hat{a}^{2}(3 q-2 x)+x\left(4 q^{2}-9 q x+6 x^{2}\right)}{x(3 x-4 q)} .
\end{aligned}
$$


Only the angular momentum of the particle depends on the black hole's spin $\hat{a}$ : there are two possibilities to consider, positive or negative $L$ - corresponding to trajectories corotating or counterrotating with respect to the black hole. The equation determining the radial position $x=\bar{r} / M$ of the ISCO is an algebraic equation of sixth degree:

$$
\begin{aligned}
& x^{6}-12 x^{5}-6\left(\hat{a}^{2}-3 q-6\right) x^{4}-4\left(-2 \hat{a}^{2} q+7 \hat{a}^{2}+2 q^{2}+27 q\right) x^{3} \\
+ & 3\left(3 \hat{a}^{4}+10 \hat{a}^{2} q+43 q^{2}\right) x^{2}-24\left(\hat{a}^{4} q+4 \hat{a}^{2} q^{2}+3 q^{3}\right) x+16 q^{2}\left(\hat{a}^{2}+q\right)=0 .
\end{aligned}
$$

Depending on the values of $(\hat{a}, q)$, there are up to six real solutions to the previous equation, but their analytical expressions are in general unavailable. We are interested to examine the case $q \leq 0$, distinctive of our black hole configuration, and we demand that the ISCOs lie outside the position of the external black hole horizon, located at

$$
x_{h o r}=1+\sqrt{1-\hat{a}^{2}-q} .
$$

In the remaining of this section, we study corotating and counterrotating configurations for representative choices of $q$, starting from a brief review of ISCOs for Kerr black holes $(q=0)$.

- Case $q=0$ : the Kerr black hole. The case $q=0$ reduces the system to a Kerr black hole. Equation ([2.108) simplifies considerably, and leads to two branches of physical solutions, corresponding to a corotating and a counterrotating orbit: see the textbook [2:3] for an excellent account. We plot in Figure 2.2 the radial position of the orbit $x=\bar{r} / M$ versus black hole spin $a / M$ : corotating orbits become closer and closer to the horizon as we increase the spin of the black hole (see black curve on the left panel), while counterrotating orbits become more and more distant. Corotating orbits can touch the horizon for the extremal value of the spinning parameter $a$. The binding energy of the object per unit rest mass - given by the quantity $(1-E)$ - versus black hole spin have opposite behaviour: the binding energies of corotating objects on ISCOs increase as the black hole spin increases, while the same quantity decreases for counterrotating objects. The maximal binding energy for an object in a corotating orbit around an extremal black 
hole $(a=M)$ reaches the value $1-E=0.42$, which corresponds to the maximum amount of extractable gravitational energy.

- Case $q=-0.2$ : When $q$ is small and negative, the properties of ISCO trajectories are qualitatively similar to the case of Kerr. An important difference is that corotating stable orbits can never touch the horizon, not even for extremal values of the spinning parameter, which is $a_{\max }=1.05 \mathrm{M}$ for $q=-0.2$. The binding energy for corotating ISCO never exceed values of order 20 per cent in this case. Notice that, as discussed in section 2.4 .2 .1 , the equatorial region of the black hole could rotate faster than $a_{\max }$, and still be well defined: we represent with dashed red curve the additional interval of black hole spin that would be allowed at the equator. On the other hand, the requirement of having a regular horizon everywhere, including at the poles, restricts the value of $a$ to be smaller than $a_{\max }$.

- Case $q=-5$ : When $q$ becomes more negative, the features we noticed for small $q$ become more accentuated. For $q=-5$, the maximal value of the rotation parameter is $a_{\max }=1.72 M$. Corotating ISCOs are far from the black hole horizon, even for extremal values of $a_{\max }$. The maximal value of the binding energy on a corotating ISCO is of few per cent. 

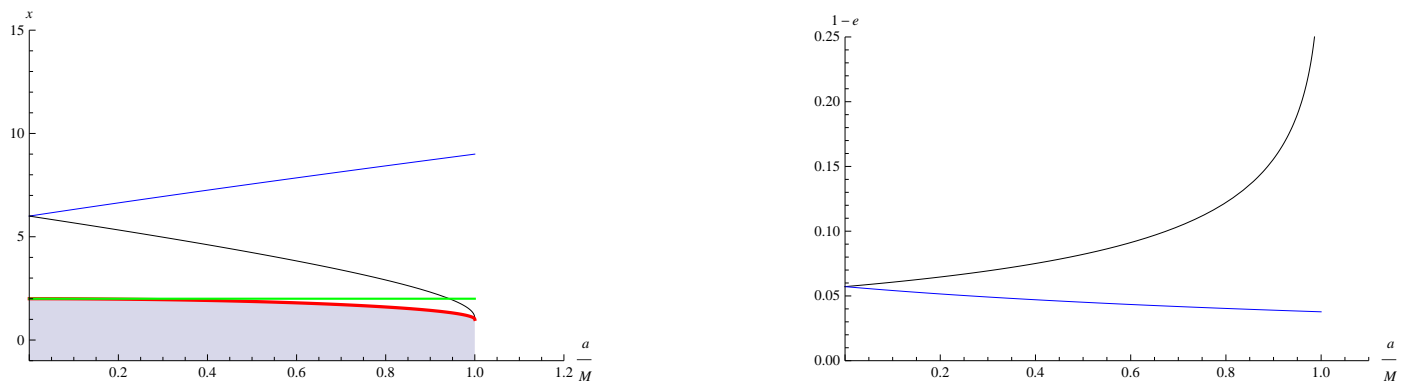

Figure 2.2: Panel on the left: Innermost circular orbits for a Kerr black hole: radial position of the orbit $x=\bar{r} / M$ versus black hole's spin a/M. There are two branches of solutions, corresponding to corotating (black) and counterrotating (blue) ISCOs. The red line corresponds to the horizon, and the shaded part the forbidden region inside the black hole horizon. The green line is the boundary of the ergosphere. Panel on the right: binding energy $1-E$ versus black hole spin $a / M$.
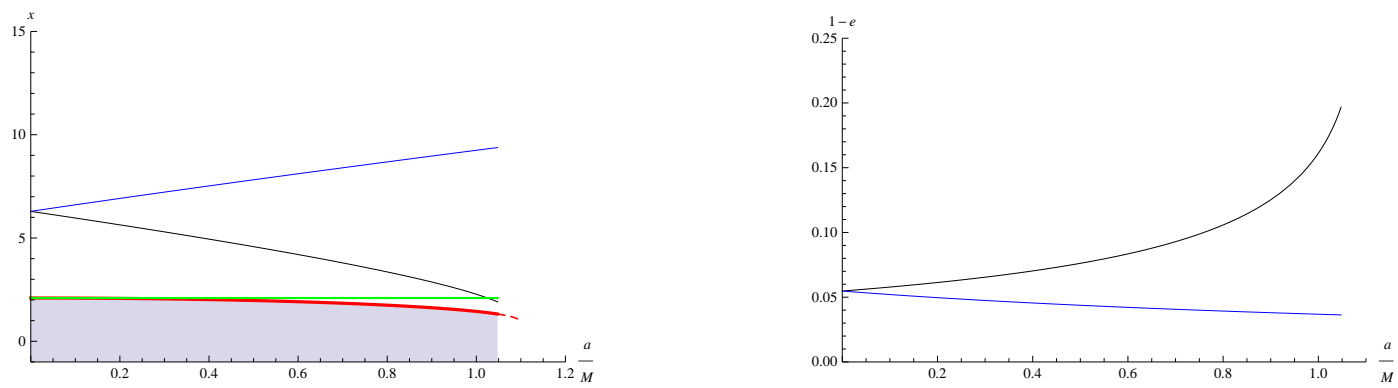

Figure 2.3: Innermost circular orbits (panel on the left) and binding energy versus black hole's spin (panel on the right) for a black hole with $q=-0.2$. Color codes as in Figure [.g. The corotating ISCO does not touch the black hole's horizon, even for extremal values of the black hole spin.
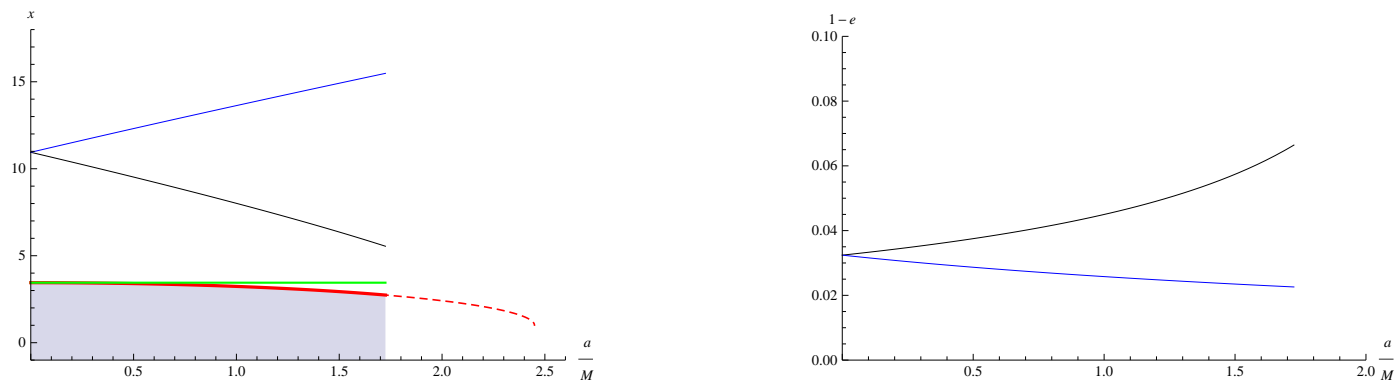

Figure 2.4: ISCOs (left) and binding energies (right) versus black hole's spin, for a black hole with $q=-5$. Color codes as in Figure [2. The corotating ISCO neither touches the black hole's horizon nor the ergosphere, even for extremal values of the black hole's spin. 


\section{A NEW ROTATING BLACK HOLE SOLUTION}

In conclusion, we think that our findings might be relevant for studying extraction of rotational energy from the disformal black hole but that would require a long and technical analysis for an exhaustive treatment, which is beyond the scope of this work (see for example [125] for further discussions about energy extraction mechanisms). However, from what we have already studied, we can make some qualitatively basics considerations. For large values of $|\beta|$, the size of the ergoregion can be greater than for Kerr black holes: at the equator, the radial size of the ergosphere is

$$
r_{e r g}=M\left(1+\sqrt{1+\frac{Q^{2}}{M^{2}}\left(\beta^{2}-1\right)}\right)
$$

hence it can be well larger than $2 M$ for $|\beta| \gg 1$. Massive particles in the ergosphere can have negative energy [23]. This implies that a Penrose process [30, 41] can be in principle devised: a massive object - e.g. a star - can break into two fragments through tidal effects within the ergosphere. One part - with negative energy falls into the black hole; the other part escapes, carrying away more energy than the initial object, and slowing down the black hole rotation. Since the ergosphere region can be large in our set-up, it might be easier to extract energy through this process ${ }^{\square}$. Other mechanisms for energy extraction can be applied in our context, as black hole superradiance [129] or some version of the Blandford-Znajek mechanism [217], possibly using our vector interactions. In fact, it is known that the efficiency of energy extraction can be increased for charged black holes [218]. Moreover, as we have learned around equation (2.102), for our configurations the angular velocity of massive particles on circular orbits can be parametrically larger than in Kerr, possibly making more efficient the mechanism behind the idea of black hole colliders [219]. As said, these are just qualitatively considerations, and it would be interesting to explore them in more details with future further studies.

\footnotetext{
${ }^{1}$ Although it might be not too efficient since only unstable circular orbits are contained in the ergoregion, and the falling objects might not find the correct orbital configurations to make the mechanism feasible.
} 


\subsubsection{Case 3: $A_{r}(r), A_{\theta}(\theta)$}

For this case, we consider a gauge vector $A_{\mu}$ with both non-vanishing radial and polar components; to satisfy the condition ( $\mathbb{Z} \cdot \mathrm{Ig})$, we choose $A_{r}$ to be a function of $r$ only, while $A_{\theta}$ is chosen to be a function of $\theta$ only:

$$
A_{r}(r), \quad A_{\theta}(\theta) .
$$

As we have already seen in the previous case, the radial and the polar profiles play a crucial role in the spacetime geometry, since they affect the position of both the horizon and the singularity. With the choice ([.110), the metric reads

$$
\begin{aligned}
d s^{2}= & g_{t t} d t^{2}+g_{r r} d r^{2}+g_{\theta \theta} d \theta^{2}+g_{\varphi \varphi} d \varphi^{2} \\
& +2\left(g_{t r} d t d r+g_{t \theta} d t d \theta+g_{t \varphi} d t d \varphi+g_{r \theta} d r d \theta+g_{r \varphi} d r d \varphi+g_{\theta \varphi} d \theta d \varphi\right),
\end{aligned}
$$

with components

$$
\begin{aligned}
& g_{t t}=-1+\frac{\beta^{2} Q^{2} r^{2}-\rho^{2}\left(Q^{2}-2 M r\right)}{\rho^{4}}, \\
& g_{r r}=\frac{\rho^{2}}{\Delta}+\beta^{2} A_{r}(r)^{2}, \\
& g_{\theta \theta}=\rho^{2}+\beta^{2} A_{\theta}(\theta)^{2}, \\
& g_{\varphi \varphi}=\sin ^{2} \theta\left[a^{2}+r^{2}-\frac{a^{2} \sin ^{2} \theta\left(Q^{2}-2 M r\right)}{\rho^{2}}+\frac{\beta^{2} a^{2} \sin ^{2} \theta Q^{2} r^{2}}{\rho^{4}}\right], \\
& g_{t r}=-\frac{\beta^{2} Q r A_{r}(r)}{\rho^{2}}, \\
& g_{t \theta}=-\frac{\beta^{2} Q r A_{\theta}(\theta)}{\rho^{2}}, \\
& g_{t \varphi}=\frac{a \sin ^{2} \theta\left[\rho^{2}\left(Q^{2}-2 M r\right)-\beta^{2} Q^{2} r^{2}\right]}{\rho^{4}}, \\
& g_{r \theta}=\beta^{2} A_{r}(r) A_{\theta}(\theta), \\
& g_{r \varphi}=\frac{\beta^{2} a \sin ^{2} \theta Q r A_{r}(r)}{\rho^{2}}, \\
& g_{\theta \varphi}=\frac{\beta^{2} a \sin ^{2} \theta Q r A_{\theta}(\theta)}{\rho^{2}} .
\end{aligned}
$$




\section{A NEW ROTATING BLACK HOLE SOLUTION}

As we did in the previous cases, we try to determine the event horizon position by looking at the hypersurface such that

$$
g^{r r}=0
$$

Moreover, to be sure of the regularity of the geometry, we have also to check that spacetime singularities (if any exist) are within the event horizon; so, we fix both $A_{r}$ and $A_{\theta}$ to have a disformal ${ }^{\square}$ horizon and a geometry everywhere regular on its exterior, and we find

$$
\begin{aligned}
& A_{r}=\frac{i \sqrt{\left(r^{2}-1\right)\left(a^{2}+r(r-2 M)+Q^{2}\right)+\beta^{2} Q^{2} r^{2}}}{\beta \Delta}, \\
& A_{\theta}=\frac{i a \cos ^{2} \theta}{\beta^{2}} .
\end{aligned}
$$

With these particular choices, we get a spherical (in Boyer-Linquist coordinates) event horizon with radius

$$
r_{H}=M+\sqrt{M^{2}-a^{2}-Q^{2}\left(1-\beta^{2}\right)} .
$$

Compared with the GR result

$$
r^{K N}=M+\sqrt{M^{2}-a^{2}-Q^{2}}
$$

we notice that the disformal transformation acts like a "distortion" of the charge $Q$, without really modifying the structure and the shape of the horizon. Consequently, every physical result related with the event horizon radius still holds, with just a modification of the charge $Q$ with the disformed one $Q_{\beta}=Q\left(1-\beta^{2}\right)$. As in

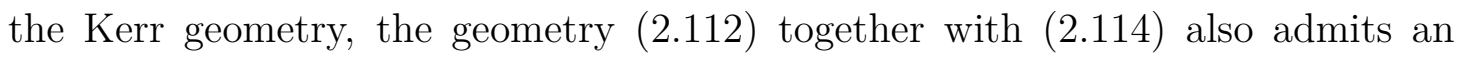
ergosphere, bounded by the ergosurface located at

$$
k^{\mu} k_{\mu}=0: \quad\left(r^{2}+a^{2} \cos ^{2} \theta\right)\left(r^{2}+a^{2} \cos ^{2} \theta-2 M r+Q^{2}\right)-\beta^{2} Q^{2} r^{2}=0 .
$$

\footnotetext{
${ }^{1}$ Interestingly, we found that there are no possible choices for $A_{r}$ and $A_{\theta}$ but the trivial one $\left(A_{r}=A_{\theta}=0\right)$ such that the event horizon coincides with the GR one.
} 


\subsection{New rotating solutions in vector-tensor theories of gravity}

It should be noticed that the above expression for the ergosurface coincides with the ones found in the two previous cases ([2.4.D), ([2.4.2); this was expected, since the ergosurface location only depends on the time component of the gauge vector, which is the same in all the cases studied up to now. On the contrary, the radial and the angular components both affect the position of singularities; with the choice ( $(\mathbb{2 . 1 4})$, we find that the as in the GR case the geometry has a singularity inside the event horizon located at

$$
r^{2}+a^{2} \cos ^{2} \theta=0
$$

Compared with the two cases studied in the previous sections, both the radial and the polar component of the gauge vector look deeply different, since (in this case) they are both imaginary and proportional to $1 / \beta$. The fact that the disformal parameter $\beta$ appears at the denominator in the equations (2.14) and hence it makes the two components divergent in the limit $\beta^{2} \rightarrow 0$ may be look odd: however, we have checked that all the geometric quantities recover the GR results when $\beta$ approaches zero, and since in that limit the Abelian $U(1)$ gauge symmetry is restored we can use gauge freedom to "absorb" the divergences appearing in both the radial and the polar vector profiles $A_{r}$ and $A_{\theta}$.

However, from (2.114) we noticed that both $A_{r}$ and $A_{\theta}$ profiles are imaginary. In the Einstein-Maxwell case, since the theory is gauge invariant and $A_{\mu}$ is not a physical observable, there is no need to require that $A_{\mu}$ is real, but the physical observable quantities (such has the magnetic field) have to be. In our case, after the disformal transformation is applied, the radial and polar profile of the gauge vector $A_{\mu}$ become crucial for the spacetime structure, so it is reasonable to think that they lead to some observable quantities. Furthermore, we have checked that the associated magnetic field is not real. Following these considerations, we think it is reasonable to reject the metric solution associated with (2.14), even though it could be interesting to be studied for some speculative purposes.

\subsubsection{Case 4: $A_{r}(r, \theta), A_{\theta}(r, \theta)$}

At the end, let's study the most general case with both $A_{r}(r, \theta)$ and $A_{\theta}(r, \theta)$ being functions of both $r$ and $\theta$ and satisfying the condition ( $[. \perp \mathrm{g})$. With generic radial 
and polar gauge vector components, the metric reads

$$
\begin{aligned}
d s^{2}= & g_{t t} d t^{2}+g_{r r} d r^{2}+g_{\theta \theta} d \theta^{2}+g_{\varphi \varphi} d \varphi^{2} \\
& +2\left(g_{t r} d t d r+g_{t \theta} d t d \theta+g_{t \varphi} d t d \varphi+g_{r \theta} d r d \theta+g_{r \varphi} d r d \varphi+g_{\theta \varphi} d \theta d \varphi\right)
\end{aligned}
$$

with components

$$
\begin{aligned}
g_{t t} & =-1+\frac{\beta^{2} Q^{2} r^{2}-\rho^{2}\left(Q^{2}-2 M r\right)}{\rho^{4}} \\
g_{r r} & =\frac{\rho^{2}}{\Delta}+\beta^{2} A_{r}(r, \theta)^{2} \\
g_{\theta \theta} & =\rho^{2}+\beta^{2} A_{\theta}(r, \theta)^{2} \\
g_{\varphi \varphi} & =\sin ^{2} \theta\left[a^{2}+r^{2}-\frac{a^{2} \sin ^{2} \theta\left(Q^{2}-2 M r\right)}{\rho^{2}}+\frac{\beta^{2} a^{2} \sin ^{2} \theta Q^{2} r^{2}}{\rho^{4}}\right] \\
g_{t r} & =-\frac{\beta^{2} Q r A_{r}(r, \theta)}{\rho^{2}} \\
g_{t \theta} & =-\frac{\beta^{2} Q r A_{\theta}(r, \theta)}{\rho^{2}} \\
g_{t \varphi} & =\frac{a \sin ^{2} \theta\left[\rho^{2}\left(Q^{2}-2 M r\right)-\beta^{2} Q^{2} r^{2}\right]}{\rho^{4}} \\
g_{r \theta} & =\beta^{2} A_{r}(r, \theta) A_{\theta}(r, \theta) \\
g_{r \varphi} & =\frac{\beta^{2} a \sin ^{2} \theta Q r A_{r}(r, \theta)}{\rho^{2}} \\
g_{\theta \varphi}= & \frac{\beta^{2} a \sin ^{2} \theta Q r A_{\theta}(r, \theta)}{\rho^{2}} .
\end{aligned}
$$

Again, we determine the horizon position by looking at the $g^{r r}$ metric component:

$$
g^{r r}=\frac{\Delta}{\rho^{2}} \frac{\rho^{2} \Delta-\beta^{2}\left[Q^{2} r^{2}-4 \Delta \sin ^{2} \theta \cos ^{2} \theta A_{\theta}(r, \theta)^{2}\right]}{\rho^{2} \Delta-\beta^{2}\left[Q^{2} r^{2}-4 \Delta \sin ^{2} \theta \cos ^{2} \theta A_{\theta}(r, \theta)^{2}-\Delta^{2} A_{r}(r, \theta)^{2}\right]}
$$

From the above expression, it is therefore clear that the choice of both $A_{r}$ and $A_{\theta}$ is crucial for the resulting geometry; moreover, it easy to recover the different special cases we have already studied in the previous sections. For example, the choice $A_{r}=A_{\theta}=0$ leads to

$$
g^{r r}=\frac{\Delta}{\rho^{2}}
$$


which is the GR result and hence it admits the Kerr-Newman horizon, as shown in ([.4. d): however, we now know it is not a physical solution, since it has a naked singularity. Instead, with $A_{\theta}=0$ and $A_{r}(r)$ we are left with

$$
g^{r r}=\frac{\Delta}{\rho^{2}} \frac{\rho^{2} \Delta-\beta^{2} Q^{2} r^{2}}{\rho^{2} \Delta-\beta^{2}\left[Q^{2} r^{2}-\Delta^{2} A_{r}(r)^{2}\right]},
$$

which motivates the particular solution

$$
A_{r}=\frac{Q r}{\Delta}
$$

we studied in ( 2.42$)$, in order to have

$$
g^{r r}=\frac{\rho^{2} \Delta-\beta^{2} Q^{2} r^{2}}{\rho^{4}},
$$

which admits a non spherical event horizon ([2.76]).

To have a physical black hole solution, together with the existence of an event horizon we have already discussed about the necessity of avoiding naked singularities. To be sure of the regularity of the geometry, we have to check that spacetime singularities are within the event horizon; since the singularities arise where the metric determinant vanishes, it is useful to compute it:

$$
g=\frac{\rho^{2}}{\Delta} \frac{-\beta^{2} \Delta\left[A_{r}(r, \theta)^{2} \Delta+4 \sin ^{2} \theta \cos ^{2} \theta A_{\theta}(r, \theta)^{2}\right]+\beta^{2} Q^{2} r^{2}-\rho^{2} \Delta}{4 \cos ^{2} \theta} .
$$

Again, it is evident how the radial and the polar components of the gauge vector $A_{\mu}$ determine the spacetime structure, shaping both the horizon and the singularity. So, we can use ([.12]) and ([2.126]) to find particular choices for both $A_{r}$ and $A_{\theta}$ in order to obtain regular black hole solutions different from the GR ones. However, it seems that for the radial and polar vector components there are no other simple and interesting choices more than the ones we have already discussed. In fact, in our understanding, there are no choices with a non vanishing, real $A_{\theta}$ profile leading to regular black hole solutions which recover the GR ones when the disformal coupling is turned off. In most cases, the $A_{r}$ and $A_{\theta}$ profiles necessary to make the geometry regular do not satisfy the condition ( $2 . \perp \mathrm{M})$. 


\section{A NEW ROTATING BLACK HOLE SOLUTION}

\subsection{Discussion}

In this chapter we obtained and studied exact solutions for rotating black holes in a specific example of vector Galileons, a theory of modified gravity motivated by the dark energy problem, that involves additional vector degrees of freedom besides a spin-2 graviton. The set-up has many features in common with several modified gravity models, including derivative self-couplings and non-minimal couplings with gravity. We determined a new family of black hole solutions applying an appropriate disformal transformation to a system related to the Kerr-Newman solution of Einstein-Maxwell gravity, and discussed various physical implications that differentiate this system from known rotating solutions in General Relativity. In particular, we used the $U(1)$ gauge vector of Einstein-Maxwell gravity as the disformal vector of the disformal transformation. Interestingly, we found that regular black hole solutions are generated only for particular choices of the disformal vector. In fact, the existence of regular geometries depends on the specific choice of the radial and polar components of the gauge vector, since the gauge symmetry is manifestly broken after the disformal transformation is applied. Remarkably, we have been able to find a particular choice for the gauge vector which generates a family of regular black holes with disformal horizons, namely event horizons whose deviations from the GR ones are parametrically controlled by the disformal coupling.

The rotating black hole configurations we determined are valid also for large values of the black hole's spin parameter. Their deviation from a Kerr-Newman solution is parametrically controlled by a dimensionless quantity associated with non-minimal couplings between vectors and gravity. The black holes are characterised by three asymptotic conserved quantities: mass, spin, and vector charge. The black hole's horizon is oblate in Boyer-Lindquist coordinates, since its radial position depends on the polar coordinates: this makes a difference with KerrNewman solutions, whose horizon is at fixed radius in Boyer-Lindquist coordinates. We showed that the maximal value for the black hole's spin can be parametrically larger than in the Kerr-Newman family of solutions, for the same value of asymptotic charges. We then studied equatorial circular trajectories around our solutions, 
which admit an analytical treatment, and we showed that probe massive objects can rotate faster than around Kerr-Newman configurations. Moreover, innermost stable circular orbits (ISCOs) lie far away from the black hole horizon with respect to rotating black holes in GR. It will be also interesting to determine whether there exists an analogue of the Carter constant [220]], which allows us to integrate the equations for time-like geodesics also beyond the case of circular equatorial orbits we studied here.

Another possible interesting development about the oblate horizon and the ISCOs of the black hole solution we got, concerns black hole shadows due to the lensing of photons travelling nearby the horizon, with an impact parameter close to the critical one [221, 222]. Remarkably, the recent results from the Event Horizon Telescope [31-36] confirmed that predicted black hole shadows are actually observable and measurable, and possible deviation from predictions could therefore reveal new features of astrophysical black holes. Consequently, a new interest for black hole shadows in modified gravity [186, 22:3] has recently come out, hoping to get more black hole "pictures" like the one in [3] , to be used as a testing tool for gravity theories. As an example, we mention [224] as an attempt to investigate the shadow of the disformal black hole solutions we have studied.

Further, the analysis of possible mechanisms for extracting rotational energy from the black hole, possibly exploiting vector interactions, could reveal new interesting features of such non-GR black hole solutions. Finally, the issue of stability of our configurations is an open issue that will need to be addressed for understanding the physical relevance of these objects.

In general, we stress the importance of disformal transformations as a tool to "generate" gravity theories qualitatively different from the ones on which the transformation has been applied, leading to the possibility of analytically studying black hole solutions in a larger family of gravity theories [225-227], thus opening new opportunities for finding ways to test these theories against astrophysical or cosmological observations. 


\subsection{Appendix: mass, angular momentum and charge}

In this appendix, we give a short derivation of the three fundamental quantities $(M, a, Q)$ which characterise the black hole solution we have studied. The black hole mass $M$, the rotation parameter $a$ and the charge $Q$ can be formally obtained as Komar charges, namely conserved quantities (at infinity) which arise from symmetries of the system; for more details, see [2.3]. For stationary geometries, that is spacetimes with a timelike Killing vector at spatial infinity, it is possible to define a spacetime energy as the Komar integral associate with the timelike Killing vector $k^{\mu}=\{1,0,0,0\}$. Given the Ricci tensor $R^{\mu \nu}$, we define a current

$$
J_{t}^{\mu}=k_{\nu} R^{\mu \nu}
$$

which is conserved by means of the Killing condition $\nabla_{[\mu} k_{\nu]}=0$ and of the Bianchi identity. We can use the current $J^{\mu}$ to define a conserved quantity at spatial infinity as:

$$
E_{B H}=\int_{\Sigma} d^{3} x \sqrt{\gamma^{(3)}} n_{\mu} J_{M}^{\mu}
$$

where $\Sigma$ is a space-like hypersurface with induced metric $\gamma_{i j}^{(3)}$ and $n_{\mu}$ is the unit normal vector to $\Sigma$. Using Stokes theorem, and the properties of Killing vectors, we can rewrite this quantity as

$$
E_{B H}=\int_{\partial \Sigma} d^{2} x \sqrt{\gamma^{(2)}} n_{\mu} \sigma_{\nu} \nabla^{\mu} k^{\nu}
$$

where the boundary $\partial \Sigma$ has metric $\gamma_{i j}^{(2)}$ and an outward-pointing normal vector $\sigma^{\mu}$. For GR black holes (Schwarzschild, RN, Kerr, KN), the energy $E_{B H}$ corresponds to the intrinsic gravitational energy of the spacetime, namely the black hole's mass; in our case, a computation of ([2.2.29) for the black hole solution ([2.68) gives $E_{M}=M$, and hence the parameter $M$ entering in the geometry corresponds to the gravitational mass of the object, as it happens in GR.

Similarly, starting from the Killing vector $r^{\mu}=\{0,0,0,1\}$ associated with 
rotational symmetry around the coordinate $\varphi$, we can define the current

$$
J_{\phi}^{\mu}=r_{\nu} R^{\mu \nu}
$$

As we did for the black hole mass, by the Stokes theorem we can define an asymptotic charge

$$
J_{B H}=-\frac{1}{2} \int_{\partial \Sigma} d^{2} x \sqrt{\gamma^{(2)}} n_{\mu} \sigma_{\nu} \nabla^{\mu} r^{\nu}
$$

which we evaluated for the black hole configuration (2.68) to find $J_{B H}=a M$, as in GR. Therefore, $J_{B H}$ is the black hole conserved angular momentum, and $a$ is the spinning parameter defined as

$$
a=\frac{J_{B H}}{M}
$$

Finally, using the 4-vector electric current

$$
J_{Q}^{\mu}=\nabla_{\nu} F^{\mu \nu}
$$

with $F_{\mu \nu}$ being the electromagnetic field strength, we can define a conserved electric charge

$$
Q_{B H}=-\int_{\partial \Sigma} d^{2} x \sqrt{\gamma^{(2)}} n_{\mu} \sigma_{\nu} F^{\mu \nu}
$$

which in our case gives, as in GR, $Q_{B H}=Q$, which can be interpreted as the black hole electric charge. Again, we have been referring to the electric case, but we could have been more generic considering generic $U(1)$ symmetries with the corresponding vector charges.

To sum up, we find three conserved asymptotic quantities with a clear physical interpretation, which can be associated with the physical parameters describing the geometry. Moreover, these results confirm that the geometry is not affected by the disformal transformation at spatial infinity, being the conserved charges identical to the GR ones, as one would expect considering that the additional contribution from the disformal transformation goes to infinity faster than the GR one. 


\section{Chapter 3}

\section{Scalar Clouds}

Black hole superradiance is a fascinating and largely studied topic in modern astrophysics and General Relativity. When coupled to rotating black holes, scalar fields can trigger superradiance, extracting rotational energy from the black hole and slowing it down. Under some particular circumstances, the superradiant instability may form scalar clouds in the black hole's surroundings, namely stationary configurations of the scalar field whose frequencies correspond to the resonant frequencies of the superradiant gain factor. In this chapter, we extend the work done in [228] to the case of an electromagnetic Kerr-Newman black hole with both electric and magnetic charge coupled to a massive scalar field. We find a stationary scalar cloud in the black hole's surroundings, whose resonant frequencies depend on the black hole's mass, the rotational parameter, both the electric and magnetic charges and on the scalar field's mass.

\subsection{Introduction and motivations}

Since the first Gravitational Wave detection GW150914 [3], black holes are no longer simply fascinating theoretical objects predicted one century ago by one of the most elegant and revolutionary physical theories ever published, but they have rather become outstanding "open sky" laboratories to test physical phenomena, and eventually to reveal unexpected ones [2, 5, 151, 84, ए70]. In particular, the Gravitational Wave detection from a binary system of two rotating black holes, 
spiralling and finally merging into a single new black hole, has opened the doors to a new era in studying gravity, allowing us to directly have access to strong regime gravity for the first time [22.9, [230]. Without any doubt, this remarkable step has completely changed our approach to gravity tests and, in future, it could eventually lead to even more outstanding results. In fact, although the General Relativity theory has already passed a large number of incredibly accurate tests at Solar System physical scale, strong gravity effects not detectable at Solar System scale could instead be detected in the context of black hole physics, giving us even more confirmations on GR or leading us to insights into other compatible strong gravity theories [2, 84, 231-233]. Thus, in the last years the study of black holes and their related astrophysical phenomena has gained much attention and popularity, strongly encouraged and supported by an extraordinary improvement of experimental sensitivity and accuracy. Moreover, the large number of projects and proposal for future interferometers and telescopes (LISA [2:34], KAGRA [2:35-2:37], Einstein-Telescope [238, [23:] ]) and the announced upgrade of the already existing ones (Advanced LIGO [240], Advanced VIRGO [241]) gives fuel to a more than ever active scientific community.

As we said, this golden era of gravitational waves detection [37] and multimessenger astronomy [86, 242] gives us the possibility to explore a previously unaccessible region of high energy physics; therefore, it comes the need of studying and understanding high energy phenomena related with black hole physics and other relativistic compact objects. In particular, black holes could be used to test cosmological theories alternative to GR, proposed to solve important open questions about the $\mathrm{CMB}$ radiation, dark energy and dark matter; specifically, the dark energy and dark matter problems appear to be quite good candidates to find answers for, since they could both suggest the existence of ultralight scalar particles outside the standard model which, as we will see soon, have interesting interactions with black holes. In fact, in the last decades a large number of theories and proposals about GR modifications has come out [8], with the aim of reproducing GR's already tested effects and solving (or at least improving) the current limits and weaknesses of Einstein's theory. [243] 
In the plethora of modified gravity theories, a large and well studied family is represented by scalar-tensor theories, where an extra scalar field (massive or massless, real or complex, dynamical or not: there are plenty of options!) is non minimally coupled to gravity [87, 244]. As already discussed in section $\mathbb{1 0 . 3}$, such scalar fields can either be motivated with cosmology [91, 245], particle physics [14] or string theory [96, 97]. Thus, even though the particular features of each theory depend on the scalar field's nature and on the specific model, the study of scalar fields in strong gravity scenarios has attracted the attention of the scientific community. In particular, a lot of work has been done on the interaction of scalar fields with black holes, leading to interesting and sometimes quite unexpected results. Among these, we have already discussed about black hole superradiance in chapter $\square$. As it should be clear at this point, superradiance is both a classical and quantum effect which causes an energy enhancement of a reflected wave/particle after a scattering event in the presence of a dissipative mechanism. The energy enhancement of the reflected wave is usually provided by an energy reduction of the target body, where the energy is transferred to the scattered wave during the collisions and due to the presence of dissipative mechanism. Therefore, after the collision the reflected wave has more energy than it had before the scattering, and that is superradiance. As already explained, superradiance naturally manifests when a scalar field impinges on a black hole, provided that the black hole has available energy to be transferred to the reflected scalar wave/particle. From thermodynamics considerations, we know that the simplest black hole configuration, namely the Schwarzschild one, has an irreducible total energy (neglecting quantum effects like Hawking's evaporation) which corresponds with the total mass of the black hole itself (see [246] for a review). This means that the black hole mass represents the total gravitational energy, and in this case it cannot be reduced: hence, Schwarzschild black holes cannot support superradiant effects.

However, if the black hole rotates or if it has an intrinsic charge (such as the electromagnetic one), the total black hole energy is given by both the gravitational energy coming from the mass and the energy contribution coming from the rotation and/or charge. In this case, the irreducible energy does not correspond to the total black hole energy, and it is therefore possible to extract part of the total energy: the first and simplest mechanism found to extract the black hole energy 
is the Penrose process [4]]. Thus, a scalar field scattering off a rotating or charge black hole can trigger the superradiance (which is sometimes described like a wave version of the Penrose process), and the reflected scalar wave effectively extracts energy from the black hole.

Considering an incident scalar wave with frequency $\omega$ and angular azimuthal number $m$ upon a rotating black hole with angular velocity $\Omega_{H}$, the superradiance is achieved in the regime $\omega>m \Omega_{H}$ [125]. Moreover, as it has been already shown in [1:31, [1.33, 247], in the case of a massive scalar field scattering off a rotating black hole it is possible to trigger an instability, namely an exponentially increasing scalar density around the black hole which eventually ends in a violent emission of energy commonly known as black hole bomb.

As already discussed, the condition to develop an instability and a black hole bomb is the presence of a screening mechanism surrounding the back hole, causing the waves reflected by the horizon to be trapped in between the horizon and the mirror, bouncing back and forth from the one to the other. In the superradiant regime, every time the wave is reflected by the horizon its energy increases: therefore, if the surrounding mirror is close enough, in the journey from the horizon to the mirror there is no energy decay of the wave, resulting in a exponential increase of the scalar field energy density. When the energy density can no longer be confined, a violent release of energy is developed and hence the name "black hole bomb", which partially depletes the scalar cloud around the black hole: during this process, the black hole loses part of its rotational energy due to superradiance. Quite remarkably, this scenario is not simply a totally unphysical toy model. In fact, an effective surrounding mirror can be obtained when matter is taken into account [135, 141, 248]; moreover, a massive scalar field can naturally provide a screening mechanism, since the mass term generates a Yukawa potential which screens the low frequencies such that $\omega<\mu$, where $\mu$ is the scalar field's mass. In particular, both analytical and numerical [247, 249] studies have shown that the instability most effective regime is

$$
a \simeq M, \quad M \mu \simeq \frac{1}{2}, \quad \omega \simeq \mu, \quad \omega \simeq m \Omega_{H}
$$

where $a$ and $M$ are respectively the black hole's spinning parameter and the mass. 
Remarkably, these instabilities can end up in a stable, stationary scalar field configuration around the black hole, usually referred to as scalar cloud [247].

As it has been already explained in the theory review chapter, there is a quite large zoo of admissible "hair" or "clouds" around either rotating or static, charged or neutral black holes, and hence the predicted existence of such scalar field configurations should not be considered as a pure unphysical speculation. On the contrary, since no-hair-like theorems usually hold for GR-like black hole solutions, the prediction of supported stable scalar field configurations around a black hole gives us the opportunity to eventually test such theories against GR, since the detectable black hole signatures (gravitational waves, shadows, ecc.) could be affected by the presence of scalar fields surrounding the black hole, and therefore we would be - at least in principle - be able to eventually detect deviations from GR predictions.

Considering all these motivations, the prediction of scalar hairy black holes or even simply scalar clouds should be considered an interesting opportunity in the study and comprehension of gravity in strong regime. Related with black hole superradiance, in addition to the scalar hair formation as result of black hole bombs, other possible interesting cloud configurations has been found [16.5, [24.9, [2.50].

In particular, in [165, 250] a massive scalar field is coupled to a rotating Kerr black hole; for a completely analytical study, the author shows that it is convenient to consider a nearly extremal Kerr black hole, namely $a \simeq M$. Considering the scalar field as a test field and therefore neglecting its backreaction on the background spacetime, the scalar field's equation of motion is the homogenous Klein-Gordon equation with a mass term included:

$$
\left(\square-\mu^{2}\right) \phi=0
$$

with $\square=\nabla_{\nu} \nabla^{\nu}$ being the D'Alembert operator and $\mu$ being the scalar field's mass. As we will see in details in the next section, assuming a spherical coordinate system $(t, r, \theta, \varphi)$ on the Kerr background the scalar field equation is separable in its radial and angular variables [168, 25]]. 


\section{SCALAR CLOUDS}

Using the following ansatz for the scalar field

$$
\phi(t, r, \theta, \varphi)=Z(r) S(\theta) e^{-i \omega t} e^{i m \varphi},
$$

it is possible to end up with the following equations for the angular and radial functions $S(\theta)$ and $Z(r)$, respectively:

$$
\begin{aligned}
& \frac{1}{\sin \theta} \frac{\partial}{\partial \theta}\left(\sin \theta \frac{\partial S}{\partial \theta}\right)+\left[K+M^{2}\left(\mu^{2}-\omega^{2}\right) \sin ^{2} \theta-\frac{m^{2}}{\sin ^{2} \theta}\right] S=0 \\
& \Delta \frac{d}{d r}\left(\Delta \frac{d Z}{d r}\right)+\left\{H^{2}+\Delta\left[2 m M \omega-K-\mu^{2}\left(r^{2}+M^{2}\right)\right]\right\}=0
\end{aligned}
$$

with

$$
\Delta=(r-M)^{2}, \quad H=\left(r^{2}+M^{2}\right) \omega-m M,
$$

since we have chosen $a \simeq M$ and with $K$ being the separation constant arising from the separation of $(r, \theta)$ variables.

To find physical solutions, it is necessary to impose appropriate boundary conditions both on the angular and on the radial function.

On the angular one, one requires regularity conditions at the boundaries: $S(0), S(\pi)$. On the radial one, depending on the kind of solutions we are looking for, one needs the appropriate requirements. In this case, since we are interested in asymptotically flat configurations, we require that the scalar field vanishes at spatial infinity, i.e. we have to require a decaying behaviour far from the horizon. Moreover, we require that the field on the event horizon is purely ingoing. These boundary conditions set up an eigenvalue problem for the scalar field frequency $\omega$, which is generically a complex quantity. Depending on the sign of the imaginary part of the frequency, the scalar wave can be exponentially increasing $(\operatorname{Im}(\omega)>0)$ or exponentially decaying $(\operatorname{Im}(\omega)<0)$ in time. A third option is the case $\operatorname{Im}(\omega)=0$, which corresponds to a pure real frequency and hence to a stationary behaviour of the scalar wave in time domain. This is the case studied in [165, [250], where infinitely long-lived quasibounded scalar field configurations surrounding the rotating black hole have been found. In particular, in [165, [250] the author studied the specific case $\omega \simeq m \Omega_{H}$, which is fully analytically solvable and leads to remarkable solutions. It is interesting noticing that, being the superradiance condition 
$\omega<m \Omega_{H}$, the resonant solutions in $[165,[250]$ live on the edge of the superradiant regime. So, the following scenario is plausible: considering a rotating Kerr black hole interacting with a massive scalar test-field, the high frequency modes such that $\omega>m \Omega_{H}$ do not trigger superradiance, hence they can survive without causing instabilities or extracting energy from the black hole. On the other hand, low frequency modes with $\omega<m \Omega_{H}$ effectively trigger superradiance, extracting rotational energy from the black hole and eventually leading to instabilities and to black hole bombs, leaving the black hole with a residual scalar hair [247]. At the end, modes such that $\omega=m \Omega_{H}$ may survive in stationary configurations, creating a scalar cloud surrounding the black hole.

So, even for different scenarios the black hole seems to be likely to support and develop a surrounding hair or cloud, making the existence (and hopefully the detection) of scalar fields around rotating black holes an interesting and promising eventuality to investigate.

In the next section, we will analytically extend the results found in [165, [228, [24.9, [250] to the case of a dyonic, rotating black hole interacting with a massive, neutral, scalar test-field. At the end, in the conclusions section, we will discuss about possible ways to detect such scalar field effects.

\subsection{Scalar field clouds}

As already discussed, dyonic black holes can be motivated with string theory arguments [1501, [252]. Moreover, hidden $U(1)$ gauge vectors have been proposed as possible candidates for dark matter [ [ $6,[25: 3]$, and the recent results about the unlikely contribution to dark matter by primordial black holes have increased the hidden fields viability [254]. In addition, recent investigations [378] have showed that black hole solutions in gravity theories including a $U(1)$ hidden sector are formally described by the same Kerr-Newman solution of Einstein-Maxwell theory. So, as in the Einstein-Maxwell theory the $U(1)$ gauge theory is the electromagnetism and the two charges $Q$ and $P$ are considered as the electric and the magnetic charge respectively, it is possible to associate a generic $U(1)$ gauge field to a generic $U(1)$ theory with generic charges $Q_{1}$ and $Q_{2}$. However, to keep things as simple as 


\section{SCALAR CLOUDS}

possible, in this section we will consider the gravito-electromagnetic Kerr-Newman solution, but we are aware that a generalisation with a generic $U(1)$ gauge theory should not be considered a speculative exercise.

The system we consider in this chapter consists in a rotating dyonic KerrNewman black hole coupled to a real, massive, neutral scalar field. Such problem can be considered a generalisation of the following works:

- [249]: real, massive, neutral scalar field coupled to a Schwarzschild black hole;

- [165, [250]: real, massive, neutral scalar field coupled to a Kerr black hole;

- [228]: real, massive, charged scalar field coupled to an electric Kerr-Newman black hole.

The action describing the system we are considering is

$$
S=\int d^{4} x \sqrt{-g}\left[\frac{R}{4}-\frac{1}{4} F_{\mu \nu} F^{\mu \nu}-\frac{1}{2} \partial_{\mu} \phi \partial^{\mu} \phi-\mu^{2} \phi^{2}+V(\phi)\right] .
$$

Since we are interested in the effects of an external scalar field which interacts with a fixed background, we neglect the backreaction of the scalar field with the background metric, and hence we consider the scalar field only as an external field. Moreover, we neglect further contribution to the scalar field except from the mass $\mu$, and we set $V(\phi)=0$. The scalar field's equation of motion is the homogeneous, massive Klein-Gordon equation:

$$
\left(\square-\mu^{2}\right) \phi=0
$$

where $\square$ is the D'Alambertian operator defined as $\square=\frac{1}{\sqrt{-g}} \partial_{\mu}\left(\sqrt{-g} g^{\mu \nu} \partial_{\nu}\right)$. From Carter's and Teukolsky's arguments [2201, [251, 255], we know that the homogeneous equation on a Kerr-like spacetime is separable in its variables. Using a Boyer-Lindquist coordinate system $(t, r, \theta, \varphi)$, on a dyonic Kerr-Newman spacetime 
the equation (B.7) reads

$$
\begin{aligned}
& \frac{\partial}{\partial r}\left(\Delta \frac{\partial \phi}{\partial r}\right)+a^{2} \sin ^{2} \theta \frac{\partial^{2} \phi}{\partial t^{2}}-\frac{\left(r^{2}+a^{2}\right)^{2}}{\Delta} \frac{\partial^{2} \phi}{\partial t^{2}}-\frac{2 a\left(P^{2}+Q^{2}-2 M r\right)}{\Delta} \frac{\partial \phi^{2}}{\partial t \partial \varphi} \\
& +\frac{1}{\sin \theta} \frac{\partial}{\partial \theta}\left(\sin \theta \frac{\partial \phi}{\partial \theta}\right)+\frac{1}{\sin ^{2} \theta} \frac{\partial^{2} \phi}{\partial \varphi^{2}}-\frac{a^{2}}{\Delta} \frac{\partial^{2} \phi}{\partial \varphi^{2}}-\mu^{2}\left(r^{2}-a^{2} \cos ^{2} \theta\right) \phi=0
\end{aligned}
$$

with $\Delta=r^{2}+a^{2}-2 M r+Q^{2}+P^{2}$.

Moreover, we use the following ansatz for the scalar field:

$$
\phi(t, r, \theta, \varphi)=\sum_{m} \int_{\omega} R(r) S(\theta) e^{i m \varphi} e^{-i \omega t} d \omega
$$

where $m$ is the azimuthal number and $\omega$ is the scalar field frequency. This explicit dependence by the time and the angular coordinate $\varphi$ comes from the fact that the background metric is stationary and axisymmetric, i.e. there is no explicit dependence on the coordinates $t$ and $\varphi$. To avoid pedantry and to keep the notation simple, from now on we will omit the integration over the frequency spectrum and the summation on the angular modes. Inserting the ansatz (B.9) in the KleinGordon equation (B.8), on the Left-Hand-Side (LHS) we get

$$
e^{i(m \varphi-\omega t)} R(r) S(\theta)\left[\frac{\Delta R^{\prime \prime}(r)+2(-M+r) R^{\prime}(r)}{R(r)}+\frac{p(r)}{\Delta}+\frac{S^{\prime \prime}(\theta)+\cot \theta S^{\prime}(\theta)}{S(\theta)}+q(\theta)\right]
$$

with

$$
\begin{aligned}
p(r)= & +\frac{1}{2}\left\{2 a^{2} m^{2}+\omega^{2}\left[a^{4}+a^{2}\left(2 M r-\left(P^{2}+Q^{2}\right)+3 r^{2}\right)+2 r^{4}\right]\right. \\
& \left.+4 a m \omega\left(P^{2}+Q^{2}-2 M r\right)\right\}-\mu^{2} r^{2} \Delta
\end{aligned}
$$

and

$$
q(\theta)=\frac{1}{2}\left(a^{2} \omega^{2} \cos 2 \theta-\frac{2 m^{2}}{\sin ^{2} \theta}\right)-\mu^{2} a^{2} \cos ^{2} \theta .
$$

In order to have equation (B.J) not trivially solved, the quantity into the square brackets must vanish; since the radial and the angular part are independent, it is possible to solve the equation through separation of variables, and with a bit of 
algebra we can rewrite both the radial and the angular equation separately:

$$
\begin{gathered}
\frac{1}{\sin \theta} \frac{\partial}{\partial \theta}\left(\sin \theta \frac{\partial S}{\partial \theta}\right)+\left[\Lambda+a^{2}\left(\mu^{2}-\omega^{2}\right) \sin ^{2} \theta-\frac{m^{2}}{\sin ^{2} \theta}\right] S=0 \\
\Delta \frac{\partial}{\partial r}\left(\Delta \frac{\partial R}{\partial r}\right)+\left\{a^{2} m^{2}+2 a m \omega\left(P^{2}+Q^{2}-2 M r\right)\right. \\
\left.+\omega^{2}\left(a^{2}+r^{2}\right)^{2}-\left[\mu^{2}\left(r^{2}+a^{2}\right)+\Lambda\right] \Delta\right\} R=0,
\end{gathered}
$$

where $\Lambda$ is the separation constant arising from the separation of variables [ [167, [251]. Imposing regularity boundary conditions (at $\theta=0$ and $\theta=\pi$ ) on the angular equation, a discrete set $\left\{\lambda_{l m}\right\}$ of eigenvalues for the separation constant is picked up, described by integer indices $l$ and $m$.

The eigenfunctions of the angular equation are the spheroidal harmonics [167, 255258]

$$
S_{l m}(\gamma, \cos \theta)
$$

which are an axisymmetric generalisation of the spherical harmonics $Y_{l m}(\theta)$. In this case, the spheroidal parameter $\gamma$ has the form

$$
\gamma^{2}=a^{2}\left(\omega^{2}-\mu^{2}\right)
$$

and it parametrizes the deviation from the spherical case, which is recovered in the limit $\gamma=0$ :

$$
S_{l m}(\gamma=0, \theta)=Y_{l m}(\theta)
$$

and it is achieved (for each frequency $\omega$ ) when $a=0$, corresponding to a spherically symmetric Schwarzschild (or Reisnner-Nordström) spacetime.

Hence, in the case

$$
a^{2}\left(\omega^{2}-\mu^{2}\right) \ll 1
$$

the eigenvalues $\lambda_{l m}$ can be approximated to spherical harmonics ones [1655, 168, [256], namely

$$
\lambda_{l m} \sim l(l+1)
$$

Consequently, both the angular and the radial equation depend on $(l, m)$ indices, and we should therefore denote with $S_{l m}(\theta)$ and $R_{l m}(r)$ the angular and the radial 
solutions, respectively. However, as we did for the summation, in order to avoid pedantic notation we will omit to write the indices, unless necessary.

To solve the radial equation, it is useful to introduce the tortoise coordinate $r^{*}$

$$
r^{*}=r+\frac{2 M r_{+}-\left(P^{2}+Q^{2}\right)}{r_{+}-r_{-}} \log \left(\frac{r}{r_{+}}-1\right)-\frac{2 M r_{-}-\left(P^{2}+Q^{2}\right)}{r_{+}-r_{-}} \log \left(\frac{r}{r_{-}}-1\right),
$$

such that

$$
d r^{*}=\frac{r^{2}+a^{2}}{\Delta} d r
$$

and we define the following radial function

$$
f\left(r^{*}\right)=\sqrt{r^{2}+a^{2}} R(r) .
$$

With these redefinitions, we can rewrite the radial equation as a time-independent Schrödinger-like equation [24.9]:

$$
-\frac{d^{2} f\left(r^{*}\right)}{d r^{* 2}}+W(r) f\left(r^{*}\right)=\omega^{2} f\left(r^{*}\right)
$$

where the effective potential $W(r)$ is defined as

$$
\begin{aligned}
W(r)= & +\frac{\mu^{2} \Delta+2 a m \omega}{a^{2}+r^{2}}-\frac{a^{2} m^{2}+\left(2 a m \omega-\lambda_{l m}\right) \Delta}{\left(a^{2}+r^{2}\right)^{2}} \\
& +\frac{\left(a^{2}-4 M r+P^{2}+Q^{2}+3 r^{2}\right) \Delta}{\left(a^{2}+r^{2}\right)^{3}}-\frac{3 r^{2} \Delta^{2}}{\left(a^{2}+r^{2}\right)^{4}} .
\end{aligned}
$$

Depending on the black hole's rotation parameter and on the scalar field's mass and frequency, the effective potential $W(r)$ can act as a barrier for low frequency modes. (As an example, in the following Figure B.D and Figure $\mathbf{3 . 2}$ the effective potentials for Schwarzschild and Kerr black holes are shown for different values of the scalar field's frequencies.) 


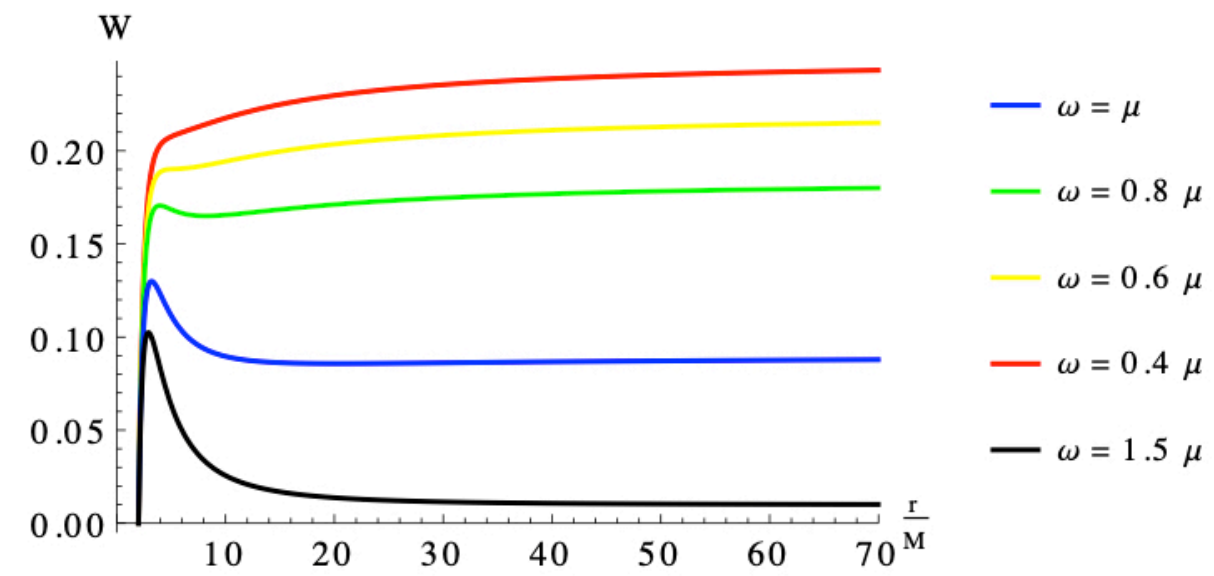

Figure 3.1: Effective potential for a Schwarzschild black hole in the case $M \mu=0.5$, with $M$ and $\mu$ being the black hole's mass and scalar field's mass respectively. On the plot, the radial coordinate $r / M=2$ corresponds to the Schwarzschild radius $r=2 M$, where the event horizon is located.

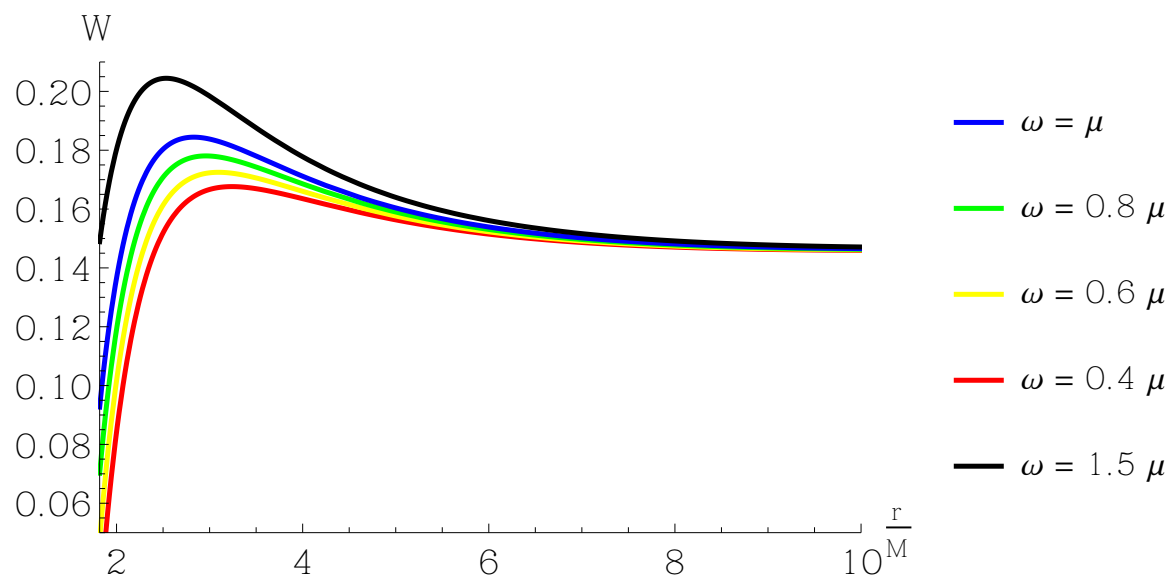

Figure 3.2: Effective potential for a Kerr black hole in the case $M \mu=0.5$, with $M$ and $\mu$ being the black hole's mass and scalar field's mass respectively. On the radial axis, the plot is truncated at the event horizon's location. 
In order to analytically solve the radial equation ([.2:3), we have to study separately the near horizon and the far horizon regions, looking for solutions which fit well in both regions (technically, we will match the near horizon solution with the far horizon one).

\subsubsection{Asymptotic region}

Far from the horizon, from the tortoise coordinate definition (B.20) we can assume $r^{*} \sim r$, and in the asymptotic limit $r^{*} \rightarrow \infty$ the radial equation simply reads

$$
\frac{d^{2} f\left(r^{*}\right)}{d r^{* 2}}+\left(\omega^{2}-\mu^{2}\right) f\left(r^{*}\right)=0
$$

The solution is given by

$$
f\left(r^{*}\right)=C_{1} e^{\sqrt{\mu^{2}-\omega^{2}} r^{*}}+C_{2} e^{-\sqrt{\mu^{2}-\omega^{2}} r^{*}},
$$

and since at large distances we have

$$
r^{*} \sim r, \quad R(r) \sim \frac{f(r)}{r}
$$

in the original radial coordinate the solution (3.26) reads

$$
R(r)=C_{1} \frac{1}{r} e^{i k_{\infty} r}+C_{2} \frac{1}{r} e^{-i k_{\infty} r},
$$

where we have defined

$$
k_{\infty}=\sqrt{\omega^{2}-\mu^{2}} .
$$

Since we are interested in decaying (bounded) solutions at spatial infinity, assuming $\omega^{2}<\mu^{2}$ we can neglect ${ }^{\mathbb{m}}$ the exponentially growing term in (3.28) and therefore we set $C_{2}=0$; now, the radial solution at infinity reads

$$
R(r)=C_{1} \frac{1}{r} e^{i k_{\infty} r}
$$

\footnotetext{
${ }^{1}$ Moreover, as explained in details in [249], in the regime $0<\omega^{2}<\mu^{2}$ it is possible to have the potential $W(r)$ in equation ([3.2.3) with a local minimum lying below $\mu^{2}$, allowing for resonance modes which tunnel through the potential barrier.
} 


\section{SCALAR CLOUDS}

which is an exponentially decaying term provided that $\omega^{2}<\mu^{2}$ and it decays as $1 / r$ in the case $\omega=\mu$. This result reflects the fact that the scalar field's mass behaves like a natural screen for low frequencies modes, stopping them from propagating to infinity.

\subsubsection{Near region}

Now we study the radial solution in the proximity of the black hole's event horizon. In the near horizon region, namely $r^{*} \rightarrow-\infty$ or equivalentely $r \rightarrow r_{+}$, it is useful to once again define a new radial coordinate:

$$
x=\frac{r-r_{+}}{r_{+}}
$$

and we also define the extremality parameter as

$$
\tau=\frac{r_{+}-r_{-}}{r_{+}}
$$

With these definitions, the horizon is located at $x=0$ and the extremal black hole configuration $r_{+}=r_{-}$corresponds to the choice $\tau=0$. Using the $x$ coordinate and the $\tau$ parameter we can rewrite the radial equation (B. 4 ) as:

$$
x(x+\tau) R^{\prime \prime}(x)+(2 x+\tau) R^{\prime}(x)+V(x) R(x)=0,
$$

where

$$
V(x)=\frac{\left[a^{2} \omega-a m+r_{+}^{2}(x+1)^{2} \omega\right]^{2}}{x(\tau+x) r_{+}^{2}}-\mu^{2}\left[a^{2}+r_{+}^{2}(x+1)^{2}\right]+2 a m \omega-\lambda_{l m} .
$$

Equations (3.3.3) and (B.34) coupled with opportune boundary conditions set up an eigenvalues problem for the field's frequencies $\omega$, which are in principle complex:

$$
\omega=\omega_{R}+i \omega_{I}
$$

Depending on the sign of the imaginary part, the modes could be exponentially decaying $\left(\omega_{I}<0\right)$ or exponentially growing $(\omega>0)$; the first scenario corresponds 
to the black hole's Quasi Normal Modes (QNMs), namely the damped oscillation frequencies of the black hole itself, while the exponentially growing scenario corresponds to the superradiant instability we have already discussed.

In our problem, we are interested in finding stationary solutions of the scalar field, namely long-lived modes supported by the black hole. This class of solution corresponds to the choice $\omega_{I}=0$, leaving us with purely real frequencies. The problem could be fully solved using numerical techniques (in [24.9] it has been solved for the spherically symmetric uncharged case): however, we are interested in finding completely analytical solutions, and so we need a further requirement. As it has been shown in [228] for a charged scalar field in an electric Kerr-Newman spacetime, for frequencies $\omega$ close to the critical superradiance frequency

$$
\omega_{c}=m \Omega=m \frac{a}{r_{+}^{2}+a^{2}}
$$

the problem is fully analytically solvable and, as expected, it admits stationary $\left(\omega_{I}=0\right)$, quasibounded (regular on the horizon and exponentially decaying at infinity), resonant $\left(\omega \sim \omega_{c}\right)$ solutions for the scalar field. Using the same terminology as in [228, 249], we will refer to these solutions as scalar clouds around rotating black holes. In fact, since we are considering a test scalar field with negligible backreaction on the spacetime, the scalar field configuration around the black hole cannot be considered an actual scalar hair (see [164] for a brief review of black holes with scalar hair); so, the scalar cloud is a stationary configuration for an external massive scalar field around a rotating black hole, rather than a full backreacting gravito-scalar spacetime. Since we are looking for stationary quasibounded resonance solutions for the scalar field around a dyonic black hole, from now on we will assume

$$
\omega \simeq \omega_{c}
$$

and with this assumption the potential $V(x)$ in equation (B.3.3) simplifies at

$$
V_{c}(x)=-\lambda_{l m}-\mu^{2}\left[a^{2}+r_{+}^{2}(x+1)^{2}\right]+2 a m \omega+\frac{r_{+}^{2}\left(x^{2}+2 x\right)^{2} \omega^{2}}{x(\tau+x)},
$$




\section{SCALAR CLOUDS}

and the radial equation becomes

$$
x(x+\tau) R^{\prime \prime}(x)+(2 x+\tau) R^{\prime}(x)+V_{c}(x) R(x)=0 .
$$

To analytically solve the last equation it is useful to study the extremal $\tau=0$ and the non extremal $\tau \neq 0$ cases separately, since the latter is quite more complicated than the former and to solve it we will use some results of the extremal case.

\subsubsection{Extremal case: $\tau=0$}

In the extremal case $\tau=0$, the radial equation (B.3.3) simply becomes

$$
x^{2} R^{\prime \prime}(x)+2 x R^{\prime}(x)+V_{c}^{0}(x) R(x)=0,
$$

with

$$
V_{c}^{0}(x)=-\lambda_{l m}-\left[a^{2}+\left(r_{+}^{2}(1+x)^{2}\right)\right] \mu^{2}+2 a m \omega+r_{+}^{2}(x+2)^{2} \omega^{2} .
$$

Equation (3.40) is analytically solved for all $x$-values, and the solution is a superposition of confluent hypergeometric functions of first type [Z56]:

$$
\begin{aligned}
R(x)= & +\tilde{c_{1}} e^{-\epsilon x} x^{-\frac{1}{2}+\beta}{ }_{1} F_{1}\left[\frac{1}{2}+\beta-\eta, 1+2 \beta, 2 \epsilon x\right] \\
& +\tilde{c_{2}} e^{-\epsilon x} x^{-\frac{1}{2}-\beta}{ }_{1} F_{1}\left[\frac{1}{2}-\beta-\eta, 1-2 \beta, 2 \epsilon x\right],
\end{aligned}
$$

where ${ }_{1} F_{1}[a, b, z]$ is the hypergeometric confluent function and we have defined

$$
\begin{aligned}
\beta & =\sqrt{\frac{1}{4}+\lambda_{l m}+\mu^{2}\left(a^{2}+r_{+}^{2}\right)-2 a m \omega-4 r_{+}^{2} \omega^{2}}, \\
\eta & =-\frac{\left(\mu^{2}-2 \omega^{2}\right) r_{+}}{\sqrt{\mu^{2}-\omega^{2}}}, \\
\epsilon & =r_{+} \sqrt{\mu^{2}-\omega^{2}}, \\
\tilde{c_{1}} & =c_{1} \frac{-2 \beta}{\Gamma\left(\frac{1}{2}-\beta-\eta\right)}+c_{2} \frac{\frac{1}{2}+\beta+\eta}{\Gamma\left(\frac{1}{2}-\beta+\eta\right) \Gamma(1+2 \beta)}, \\
\tilde{c_{2}} & =c_{2} 2^{-2 \beta} \frac{\Gamma(2 \beta)}{\Gamma\left(\frac{1}{2}-\eta+\beta\right)},
\end{aligned}
$$


with $c_{1}$ and $c_{2}$ integration constants.

In the near horizon region $x \sim 0$, we can take the limits

$$
e^{-\epsilon x} \sim 1, \quad{ }_{1} F_{1}[a, b, x] \sim 1
$$

and the radial equation simplifies at

$$
R(x)=\tilde{c_{1}} x^{-\frac{1}{2}+\beta}+\tilde{c_{2}} 2^{-2 \beta} x^{-\frac{1}{2}-\beta} .
$$

Since we are interested in solutions regular on the horizon, we impose

$$
\tilde{c_{2}}=c_{2}=0, \quad \operatorname{Re}(\beta)>\frac{1}{2}
$$

and we are left with

$$
R(x)=\tilde{c_{1}} x^{-\frac{1}{2}+\beta} .
$$

To consistency, we now have to check the behaviour of the field far from the horizon, matching the asymptotic result (B.30) with the near horizon one (B.47).

Imposing conditions ( 3.46$)$ on the equation ( $\overline{3.42}$ ) and taking the asymptotic behavior of the ${ }_{1} F_{1}$ function

$$
{ }_{1} F_{1}\left[\frac{1}{2}+\beta-\eta, 1+2 \beta, 2 \epsilon x\right] \sim \Gamma(1+2 \beta)\left[\frac{e^{2 \epsilon x}(2 \epsilon x)^{-\frac{1}{2}-\beta-\eta}}{\Gamma\left(\frac{1}{2}+\beta-\eta\right)}+\frac{(-2 \epsilon x)^{-\frac{1}{2}-\beta+\eta}}{\Gamma\left(\frac{1}{2}+\beta+\eta\right)}\right],
$$

we arrive at

$$
R(x)=\frac{c_{1}}{\Gamma\left(\frac{1}{2}-\beta+\eta\right)}\left[\frac{\Gamma\left(\frac{1}{2}+\beta+\eta\right)}{\Gamma\left(\frac{1}{2}+\beta-\eta\right)}(2 \epsilon)^{-\frac{1}{2}-\beta-\eta} e^{\epsilon x} x^{-1-\eta}+(-2 \epsilon)^{-\frac{1}{2}-\beta+\eta} e^{-\epsilon x} x^{-1+\eta}\right] .
$$

Since the near horizon solution has to match the asymptotic quasibounded solution which goes to zero at spatial infinity, we have to make the growing term in the previous equation vanishing.

Thus, since the Gamma functions has no zeros [256], we have to set

$$
\frac{1}{\Gamma\left(\frac{1}{2}+\beta-\eta\right)}=0
$$




\section{SCALAR CLOUDS}

The previous condition is satisfied at the Gamma's poles, which appear when

$$
\frac{1}{2}+\beta-\eta=n
$$

with $n$ being an integer, non-negative number.

Finally, the radial equation reads

$$
R(x)=C_{1} e^{-\epsilon x} x^{-\frac{1}{2}+\beta}{ }_{1} F_{1}\left[\frac{1}{2}+\beta-\eta, 1+2 \beta, 2 \epsilon x\right]
$$

and using the resonance condition (3.5]) we can write it in terms of the generalized Laguerre Polynomials $L_{a}^{b}(z)[256]$ as

$$
R(x)=C e^{-\epsilon x} x^{-\frac{1}{2}+\beta} L_{n}^{2 \beta}(2 \epsilon x)
$$

where we have used the relation

$$
{ }_{1} F_{1}[a, b z] \propto L_{-a}^{b-1}(z) .
$$

The resonance condition (B.5]) can be rewritten as

$$
\eta=\frac{1}{2}+\beta+n
$$

and reminding that $\operatorname{Re}(\beta)>\frac{1}{2}$ we obtain

$$
\eta>0
$$

Hence, from the definition of $\eta$ we have

$$
-\frac{\left(\mu^{2}-2 \omega^{2}\right) r_{+}}{\sqrt{\mu^{2}-\omega^{2}}}>0,
$$

and together with the condition $\omega^{2}-\mu^{2}<0$ it leads to

$$
\omega<\mu<\sqrt{2} \omega
$$

The last relation implies that for a massive scalar field there does not exist any 
static $(\omega=0)$, quasibounded, resonant solution around a dyonic KN black hole, and it shows that in the massless case $(\mu=0)$ no resonance solutions are supported.

The resonance condition (3.5.5) can be analytically solved in the regime

$$
\epsilon \ll 1
$$

which corresponds to the case $\mu \sim \omega$. In this regime, neglecting terms of order $\mathcal{O}\left(\epsilon^{3}\right)$ or higher, we have

$$
\begin{aligned}
& \mu(\epsilon)=\sqrt{\omega^{2}+\frac{\epsilon^{2}}{r_{+}^{2}}}, \\
& \eta(\epsilon)=\frac{r_{+}^{2} \omega^{2}}{\epsilon}-\epsilon, \\
& \beta^{2}(\epsilon)=\left(l+\frac{1}{2}\right)^{2}-a m \omega-4 r_{+}^{2} \omega^{2}+\frac{a m}{r_{+}^{2} \omega} \epsilon^{2},
\end{aligned}
$$

where we have used the approximation

$$
\lambda_{l m} \sim l(l+1) .
$$

So, the resonance condition leads to a polynomial equation for the variable $\epsilon$ :

$$
-r_{+}^{2} \omega^{2}+\left(n+\frac{1}{2}+\sqrt{\left(l+\frac{1}{2}\right)^{2}-a m \omega-4 r_{+}^{2} \omega^{2}}\right) \epsilon+\epsilon^{2}+\mathcal{O}\left(\epsilon^{3}\right)=0
$$

where, depending on the black hole parameters and on the indices $(n, l, m)$, an infinite family of solutions there exist:

$$
\tilde{\epsilon}_{n l m}(M, a, P, Q) \text {. }
$$

Finally, the field's masses corresponding to the resonance frequencies are given by

$$
\mu_{n l m}(M, a, P, Q)=\omega\left(1+\frac{1}{2} \tilde{\epsilon}^{2}+\mathcal{O}\left(\tilde{\epsilon}^{4}\right)\right)
$$


with

$$
\begin{aligned}
\tilde{\epsilon}=\{1 & +2 n+\sqrt{(2 l+1)^{2}-4 \omega\left(a m+4 r_{+}^{2} \omega\right)} \\
& \left.+\sqrt{\left(1+2 n+\sqrt{(2 l+1)^{2}-4 \omega\left(a m+4 r_{+}^{2} \omega\right)}\right)^{2}+16 r_{+}^{2} \omega^{2}}\right\}\left(4 r_{+} \omega\right)^{-1} .
\end{aligned}
$$

\subsubsection{General case: $\tau \neq 0$}

In the general case, it is not possible to find an analytic solution (for each $x$-value) of the radial equation

$$
x(x+\tau) R^{\prime \prime}(x)+(2 x+\tau) R^{\prime}(x)+V_{c}(x) R(x)=0 .
$$

So, we try to solve it in the limit

$$
x \ll 1,
$$

where the potential reads

$$
V_{c}^{0}(x)=-\lambda_{l m}-\mu^{2}\left(a^{2}+r_{+}^{2}\right)+2 a m \omega+k^{2} \frac{x}{x+\tau},
$$

where we have defined

$$
k=2 \omega r_{+}
$$

After performing the following redefinitions of functions and variables

$$
\begin{aligned}
& R(x)=\alpha(x) g(x), \\
& z=-\frac{x}{\tau}
\end{aligned}
$$

the radial equation becomes

$$
\begin{aligned}
& -\alpha(z)\left\{z(1-z) g^{\prime \prime}(z)+\left[\frac{2 \alpha^{\prime}(z)}{\alpha(z)} z(1-z)+(1-2 z)\right] g^{\prime}(z)\right. \\
& \left.+\left[\frac{\alpha^{\prime \prime}(z)}{\alpha(z)} z(1-z)+\frac{\alpha^{\prime}(z)}{\alpha(z)}(1-2 z)+k^{2} \frac{z}{1-z}-\tilde{V}\right] g(z)\right\}=0,
\end{aligned}
$$


where the effective potential $\tilde{V}$ is given by:

$$
\tilde{V}=-\lambda_{l m}-\mu^{2}\left(a^{2}+r_{+}^{2}\right)+2 a m \omega .
$$

Reminding the generic hypergeometric differential equation [256, [259],

$$
y(1-y) t^{\prime \prime}(y)+[c-(a+b+1) y] t^{\prime}(y)-a b t(y)=0,
$$

from equation (B.]त) we have to impose the following conditions:

$$
\begin{aligned}
& \frac{2 \alpha^{\prime}(z)}{\alpha(z)} z(1-z)+(1-2 z)=c-(a+b+1) z \\
& \frac{\alpha^{\prime \prime}(z)}{\alpha(z)} z(1-z)+\frac{\alpha^{\prime}(z)}{\alpha(z)}(1-2 z)+k^{2} \frac{z}{1-z}-\tilde{V}=-a b,
\end{aligned}
$$

which lead to

$$
\alpha(z)=(1-z)^{\frac{1}{2}(a+b+c)} z^{\frac{1}{2}(c-1)}
$$

with

$$
\begin{aligned}
& a=\frac{1}{2}+\beta-i k, \\
& b=\frac{1}{2}-\beta-i k, \\
& c=1,
\end{aligned}
$$

and with $\beta$ and $k$ defined as in (B.43) and in (B.6.9), respectively. So, using (B.70) together with (3.75) we can write the complete solution to the radial equation in the near horizon region:

$$
R(x)=\left(\frac{x+\tau}{\tau}\right){ }_{2} F_{1}\left[\frac{1}{2}+\beta-i k, \frac{1}{2}-\beta-i k, 1,-\frac{x}{\tau}\right],
$$

with ${ }_{2} F_{1}[a, b, c, z]$ the hypergeometric function of the second type [2.56] .

Far from the horizon, we have to solve the radial equation (3.66) in the limit

$$
x \gg \tau \text {. }
$$

In this limit, the radial equation is equal to the one we solved in the extremal case, 


$$
x^{2} R^{\prime \prime}(x)+2 x R^{\prime}(x)+V_{c}(x) R(x)=0,
$$

which has solution

$$
\begin{aligned}
R(x)= & +\tilde{C}_{1} e^{-\epsilon x} x^{-\frac{1}{2}+\beta}{ }_{1} F_{1}\left[\frac{1}{2}+\beta-\eta, 1+2 \beta, 2 \epsilon x\right] \\
& +\tilde{C}_{2} e^{-\epsilon x} x^{-\frac{1}{2}-\beta}{ }_{1} F_{1}\left[\frac{1}{2}-\beta-\eta, 1-2 \beta, 2 \epsilon x\right] .
\end{aligned}
$$

Following the Hod's approach [165, [250], now we have to match the near and far horizon solutions in the overlapping region

$$
\tau \ll x \ll 1
$$

To do it, we have to compare and match the Gamma functions coefficients resulting from expanding the hypergeometric functions in the far horizon region with the ones in the near horizon one; skipping some long algebraic steps (look at [2:28, [250] for an explicit similar calculation), after imposing regularity conditions on the horizon and decay at spatial infinity, as we did in the extremal case we can find the resonance condition from the gamma function's poles

$$
\eta=\frac{1}{2}+\beta+n>0
$$

Again, we find that for a massive scalar field any static, quasibounded, resonant configuration around a rotating dyonic black hole is not supported. This result is consistent with the one obtained in the extremal case, since for $\tau=0$ the overlapping condition $\tau \ll x \ll 1$ is satisfied and the far horizon region can be extended to spatial infinity. So, the condition ( 382$)$ holds for generically fast or slow rotating black holes, and the results obtained in the extremal case are extended to the generic non extremal regime. 


\subsection{Conclusions}

In this chapter it has been shown how a rotating dyonic black hole admits stationary, quasi-bounded configurations for a massive, scalar test-field surrounding the black hole. Such configurations, known as scalar (boson) clouds, are composed of the resonant modes of the scalar field interacting with the background black hole. Interestingly, assuming a coupling between the scalar field and the electromagnetic sector [260], the scalar cloud surrounding the black hole could deflect the radiation travelling near the black hole: as we will see in more details in the next chapter, this could lead to the detection of right/left polarised radiation [261]. Moreover, bosonic clouds surrounding black holes (or eventually other astrophysical compact objects which allow surrounding clouds [262, [26.3]) can affect the dynamics of a binary system, affecting the motion of small compact objects in the closeness [162, [264] and hence inducing potentially detectable signatures in the Gravitational Waves waveforms coming from EMRIs (extremal mass-ratio inspirals). In the case of a binary system, tidal perturbations can induce resonant transitions between different modes [119, 265, [266] of the cloud, with summation rules similar to the hydrogen atom system [247, [265], leading to a cloud depletion of the cloud through GWs emission [265, [266]. Quite remarkably, the superradiant configurations offer even more chances to be detected: in fact, since superradiant instabilities could lead to a lack of highly spinning black holes, an indirect observation of bosonic clouds could be given by gaps in the Regge plane [121, 125]. Moreover, non stationary clouds would slowly decay through the emission of Gravitational Waves, with detectable frequencies both in the continuous and in the stochastic background area [119, 1201, [267-269].

In conclusion, the existence of bosonic clouds surrounding rotating black holes (or even other compact objects) could be detected in several different ways; in particular, continuous/stochastic GWs emission from isolated objects or GWs emission from binary systems would give us a strong evidence of ultralight bosonic fields in the strong gravity regime, opening new frontiers in axion cosmology and particle physics and on the scalar-tensor gravity sector. 


\section{Chapter 4}

\section{Black hole hair}

Black hole physics can suggest new ways to test the existence of axions. In particular, in the last decades much work has been done to analyse the phenomenon of superradiance associated with axions in the ergoregion surrounding rotating black holes. In this chapter, based on the work [주] , we instead investigate how Chern-Simons axion couplings of the form $\phi F \tilde{F}$ and $\phi R \tilde{R}$, well motivated by particle physics and string theory, can induce long-range profiles for light axion fields around charged black holes, with or without spin. We extend known solutions describing axion hair around spherically symmetric, asymptotically flat dyonic black hole configurations, charged under $U(1)$ gauge symmetries, by including non-minimal couplings with gravity. The axion acquires a profile controlled by the black hole's conserved charges, and we analytically determine how it affects the black hole's horizon and its properties. Moreover, we find a Smarr formula applying to our configurations, and we then generalise known solutions describing axion hair around slowly rotating black hole configurations with charge. To make contact with phenomenology, we briefly study how long-range axion profiles induce a polarization dependent deflection of light rays, and the properties of ISCOs for the black hole configurations we investigate.

\subsection{Introduction}

Axions can be considered among the most well motivated candidates for physics beyond the Standard Model. Introduced in order to solve the strong CP problem 


\section{BLACK HOLE HAIR}

[92-94, 271] (see the review [14]), it was soon realised they have relevant implications for cosmology, as dark matter [106-108] or dark energy candidates [245] (see the review [9]]). Moreover, axion fields arise naturally in string theory constructions (see e.g. [96]) with a broad variety of couplings and masses, motivating the string axiverse scenario of [97]. The quest of their experimental detection is currently an active research field, see e.g. [272] for a recent pedagogical review. Very light axion fields are specially interesting dark matter candidates [273-281], and their specific properties can lead to distinctive observational consequences. In this chapter, based on [ [ZTU], we investigate the relation between the physics of axions and black holes, encouraged by the great development and opportunities given us by Gravitational Waves detectors (LIGO, VIRGO, LISA, KAGRA, etc.) and telescopes (EHT, Spektr-R, etc.). Light axions, whose Compton wavelength is of the order of a black hole's Schwarzschild radius, can deposit on the ergosphere of a rotating black hole, and cause instabilities associated with the phenomenon of black hole superradiance [30, [.32, [251]. Such instabilities can have observable implications, since axions can extract rotational energy from spinning black holes [121, 168, [247, [282] (see [125] for a comprehensive review), and lead to a distinctive emission of detectable gravitational waves ${ }^{\mathbf{m}}$ (see e.g. [119, [268]).

In chapter 3 we discussed the case of a massive scalar field in a cloud configuration surrounding a rotating, charged black hole, with frequency close to the superradiance threshold frequency $\omega_{c}=m \Omega$. Here we focus on the situation where the axion Compton wavelength is much larger than a black hole's Schwarzschild radius. In this regime, the axion $\phi$ can be considered effectively massless, and the axion Lagrangian enjoys a shift symmetry $\phi \rightarrow \phi+$ const. It is then natural to ask whether $\phi$ can develop an extended radial profile, leading to long-range axion hair around a black hole. As already discussed in section ㄷ.6, there are several no-hair arguments to overcome, starting from Bekenstein results [285, 286]; on the other hand, a key property of axion fields we can exploit is the fact that they are characterised by Chern-Simons couplings to gauge fields and gravity,

$$
\phi F \tilde{F}, \quad \phi R \tilde{R}
$$

\footnotetext{
${ }^{1}$ Possible ways to probe properties of axions with neutron stars are also being investigated, see e.g. [2622, 28:3, 284].
} 
with $\tilde{F}$ and $\tilde{R}$ respectively the Hodge dual of the gauge field strength and Riemann tensor. While the gauge Chern-Simons coupling naturally appears when dealing with anomalous symmetries of the QCD axion (see the reviews [14, 272]), the gravitational Chern-Simons coupling arises in explicit calculations in string theory constructions, see e.g. [96]. The linear Chern-Simons axion couplings in (4.D) source a non-vanishing asymptotic value for the axion fields for charged and/or rotating black holes, and allow one to avoid black hole no-hair theorems, somewhat analogously to what happens in Horndeski theories, where linear couplings with the Gauss-Bonnet invariant were found in [156, 287] to be an important ingredient for overcoming the no-hair theorem of [ $[288]$.

This chapter proceeds as follows:

- In section 4.2 we set the stage presenting the shift symmetric axion system we consider. We include additional derivative couplings of the axion field with curvature, allowed by the symmetries of the system, which can be motivated by high energy constructions. We explain in detail why the Chern-Simons couplings (4.d) are expected to induce regular axion hair around regular spherically symmetric charged black holes, and around rotating black holes (with or without charge).

- In section 4.3 we study spherically symmetric dyonic black holes, with electric and magnetic charges, equipped by a long range axion hair. We generalise known solutions [149, [150, [289] to cases where the axion has derivative couplings with the Ricci tensor, motivated in section 4.2 , showing that ChernSimons couplings can be effective in generating axion hair also in theories with non-minimal derivative couplings of axions to curvature. The axion profile has a secondary charge, i.e. it depends on the black hole's conserved charges. We work at leading order in an expansion of the relevant coupling constants, finding analytical solutions and discussing how the properties of the black hole's horizon and the basic black hole thermodynamics are modified by the axion hair. 
- In section 4.4 we consider slowly rotating charged black holes, finding new configurations with axion hair, which generalize and interpolate between known solutions [155, [290]. Interestingly, we find that the gauge and gravitational Chern-Simons couplings simultaneously play a role in characterising the axion hair and the geometry, leading to mixed contributions depending on both these parameters.

- In section 4.5 we study phenomenological applications of our findings, showing how long-range black hole axion hair can bend differently the two polarizations of light crossing in proximity of the black hole (elaborating on the methods of [ [261] ), and by studying properties of the innermost stable circular orbits (ISCOs) for our configurations. Moreover, we briefly discuss how these configurations fit in the contest of astrophysical Active Galactic Nuclei (AGNs).

\subsection{System under consideration}

\subsubsection{The action}

We consider the following action describing gravity coupled with a $U(1)$ gauge field $A_{\mu}$ (with $F_{\mu \nu}=\partial_{\mu} A_{\nu}-\partial_{\nu} A_{\mu}$ its field strength) and a pseudoscalar axion field $\phi$

$$
S=\int d^{4} x \sqrt{-g}\left[\frac{R}{4}-\frac{1}{4} F^{\mu \nu} F_{\mu \nu}-\mathcal{L}_{\phi}(\phi, \partial \phi)+\mathcal{L}_{I}\left(\phi, F_{\mu \nu}, g_{\mu \nu}\right)\right]
$$

where from now on we have set units such that $c=\hbar=G_{N}=1$ and $4 \pi \epsilon_{0}=1$. This action describes Einstein gravity coupled to a $U(1)$ Maxwell field, and a pseudoscalar axion described by the Lagrangian density $\mathcal{L}_{\phi}$ (to be specified in what follows). Moreover, the axion is non-minimally coupled to the gauge and the metric fields: such interaction terms are all included in the Lagrangian density $\mathcal{L}_{I}$, which will be soon specified. The axion can be identified with the NambuGoldstone boson of a spontaneously broken Peccei-Quinn symmetry, or with the Hodge dual of a three-form field strength which appears in string theory scenarios. In both cases, Chern-Simons couplings arise naturally; so, we consider a real, 
neutral, axion field $\phi$ coupled to a gauge field through a Chern-Simons interaction term, namely

$$
\mathcal{L}_{f}^{c s}=g_{f} \phi f_{\alpha \beta} \tilde{f}^{\alpha \beta}
$$

where $g_{f}$ is the coupling constant and $\tilde{f}^{\alpha \beta}$ are the components of the generic 2form $\tilde{f}$, defined as the Hodge dual of the 2-form $f=f_{\mu \nu} d x^{\mu} \wedge d x^{\nu}$. Explicitly, the components of the dual tensor are

$$
\tilde{f}^{\alpha \beta}=\frac{1}{2} \epsilon^{\lambda \delta \alpha \beta} f_{\lambda \delta}
$$

where $\epsilon^{\lambda \delta \alpha \beta}$ is the Levi-Civita antisymmetric tensor defined from

$$
\epsilon^{\lambda \delta \alpha \beta}=\frac{1}{\sqrt{-g}} \varepsilon^{\lambda \delta \alpha \beta},
$$

with $\varepsilon^{\lambda \delta \alpha \beta}$ being the antisymmetric Levi-Civita symbol defined as

$$
\varepsilon^{\lambda \delta \alpha \beta}= \begin{cases}+1 & \text { if } \lambda \delta \alpha \beta \text { is an even permutation of } 1234 \\ -1 & \text { if } \lambda \delta \alpha \beta \text { is an odd permutation of } 1234 \\ 0 & \text { otherwise }\end{cases}
$$

In this work, we are interested in studying an axion field non-minimally coupled to the gauge field strength and gravity through Chern-Simons interaction terms:

$$
\begin{aligned}
& \mathcal{L}_{F}^{c s}=g_{F} \phi \tilde{F}^{\alpha \beta} F_{\alpha \beta}, \\
& \mathcal{L}_{R}^{c s}=g_{R} \phi \tilde{R}^{\alpha \beta \gamma \delta} R_{\alpha \beta \gamma \delta},
\end{aligned}
$$

where the field strength's and the Riemann tensor's dual are respectively defined as

$$
\tilde{F}^{\mu \nu}=\frac{1}{2} \epsilon^{\mu \nu \rho \sigma} F_{\rho \sigma}, \quad \quad \tilde{R}^{\mu \nu \rho \sigma}=\frac{1}{2} \epsilon^{\mu \nu \alpha \beta} R_{\alpha \beta}^{\rho \sigma}
$$

and with $g_{F}$ and $g_{R}$ being the respective coupling constants.

The coupling constants of the Chern-Simons terms can be associated with the axion decay constants: for our purposes we appropriately weight those operators with powers of some fundamental mass scale (for example the Planck mass) and consider the quantities $g_{F}$ and $g_{R}$ as dimensionless free constant parameters, affecting the geometry of the spacetime we consider. Additionally, higher order operators 
coupling the axion to curvature and gauge fields could in principle be included, but since they are suppressed by higher powers of the fundamental mass scale we neglect them for simplicity (see however [2.91]). At the perturbative level, axion fields typically enjoy a shift symmetry

$$
\phi \rightarrow \phi+\text { const }
$$

which can be broken by non-perturbative effects giving a mass to the axion. For cosmological purposes - as dark energy or dark matter candidates - axions are typically very light. For the aim of this work, we consider axions whose Compton wavelength is much larger than the size of the black hole. Being the Compton wavelength $\lambda_{\text {Compton }}$ of an axion field of mass $m_{\phi}$ given by

$$
\lambda_{\text {Compton }} \simeq\left(\frac{10^{-10} \mathrm{eV}}{m_{\phi}}\right)\left(\frac{M_{B H}}{M_{\odot}}\right) r_{\mathrm{Schw}}^{\mathrm{sun}},
$$

we can assume that the axion masses are much smaller than $10^{-10} \mathrm{eV}$ when considering stellar black holes, or much smaller than $10^{-16} \mathrm{eV}$ when investigating supermassive black holes sitting in the centre of galaxies, such that $\lambda_{\text {Compton }}$ is well larger than the corresponding black hole's Schwarzschild radius. As a consequence, we will neglect shift symmetry-breaking effects, and consider the axion field as effectively massless. The shift-symmetric axion Lagrangian $\mathcal{L}_{\phi}$ we consider is quadratic in the axion field, so to respect the pseudoscalar properties of the axion. It contains the standard axion kinetic terms, plus non-minimal derivative couplings with gravity:

$$
\mathcal{L}_{\phi}=\frac{1}{2} \nabla^{\mu} \phi \nabla_{\mu} \phi-\lambda G^{\mu \nu} \nabla_{\mu} \phi \nabla_{\nu} \phi
$$

with $G^{\mu \nu}$ being the Einstein tensor. This is the most general shift-symmetric quadratic action for the pseudoscalar axion field, which is invariant under the parity symmetry properties of the pseudoscalar axion, and leads to second order equations of motion. The second contribution in (4.]) is a dimension-six operator belonging to the Horndeski action. We weight it with appropriate powers of the Planck mass, and consider the parameter $\lambda$ as dimensionless. We include the 
operator controlled by $\lambda$ for the following reasons:

i) it is allowed by the symmetries of the axion - shift symmetry and parity invariance - hence it can be expected from an effective field theory point of view, being induced by perturbative loop corrections to the tree-level action. Although derivative couplings of axions with curvature are rarely considered (see however [2.92] for an example), they can be motivated by string theory constructions. For example, [29:3] computed $\alpha^{\prime}$ and loop corrections in heterotic string systems including the 3-form antisymmetric tensor field $H_{\mu \nu \rho}$, finding various couplings of the latter with Riemann and Ricci tensors. Once the three form field is Hodge-dualised to a pseudoscalar, such couplings can lead to derivative couplings of the axion to curvature tensors ${ }^{\mathrm{m}}$, as in Lagrangian (4.1).

ii) Non-minimal couplings of scalar fields with curvature have a long history in models of dark energy (see e.g. [8] for a review) and cosmological inflation (as in Starobinsky [2.94] or Higgs inflation [2.95, 206]). Although they are typically suppressed by some high energy scale, they can have important implications when the scalar acquires a non-trivial vacuum-expectation-value (vev). Since their consequences for black hole physics and the existence of scalar hair evading strong no-hair theorems are well studied topics, it is then natural to investigate this topic for the case of pseudoscalar axions, and to explore the role of Chern-Simons couplings in allowing for black hole axion hair, including derivative couplings with gravity.

So, the complete action reads

$$
\begin{aligned}
S=\int d^{4} x \sqrt{-g}[ & \frac{R}{4}-\frac{1}{4} F^{\alpha \beta} F_{\alpha \beta}-\frac{1}{2} \partial^{\alpha} \phi \partial_{\alpha} \phi \\
& \left.-\lambda G^{\mu \nu} \partial_{\mu} \phi \partial_{\nu} \phi-\frac{g_{F}}{4} \phi F_{\alpha \beta} \tilde{F}^{\alpha \beta}+\frac{g_{R}}{4} \phi \tilde{R}^{\alpha \beta \gamma \delta} R_{\alpha \beta \gamma \delta}\right],
\end{aligned}
$$

\footnotetext{
${ }^{1}$ These are heuristic, preliminary considerations that would be interesting to further develop in a proper string theory setting; on the other hand, this goes beyond the scope of this work.
} 
and the equation of motion for the metric, the gauge vector and the axion are:

$$
\begin{gathered}
R_{\mu \nu}-\frac{1}{2} R g_{\mu \nu}=2\left(T_{\mu \nu}^{\phi}+T_{\mu \nu}^{F}+T_{\mu \nu}^{c s}+T_{\mu \nu}^{\lambda}\right), \\
\nabla_{\alpha}\left(\partial^{\alpha} A_{\mu}-\partial_{\mu} A^{\alpha}\right)-g_{F} \epsilon_{\mu \rho \alpha \beta} \partial^{\alpha} \phi \partial^{\beta} A^{\rho}=0, \\
\square \phi=\frac{1}{4}\left(g_{F} F_{\alpha \beta} \tilde{F}^{\alpha \beta}-g_{R} \tilde{R}^{\alpha \beta \gamma \delta} R_{\alpha \beta \gamma \delta}-2 \lambda G^{\alpha \beta} \nabla_{\beta} \partial_{\alpha} \phi\right),
\end{gathered}
$$

where the stress-energy tensors are defined as:

$$
\begin{aligned}
& T_{\mu \nu}^{\phi}=\partial_{\mu} \phi \partial_{\nu} \phi-\frac{1}{2} g_{\mu \nu} \partial^{\alpha} \phi \partial_{\alpha} \phi, \\
& T_{\mu \nu}^{F}=F_{\mu}{ }^{\alpha} F_{\nu \alpha}-\frac{1}{4} g_{\mu \nu} F^{\alpha \beta} F_{\alpha \beta}, \\
& T_{\mu \nu}^{c s}=-2 g_{R}\left[\partial^{\alpha} \phi \epsilon_{\alpha \beta \gamma(\mu} \nabla^{\gamma} R_{\nu)}{ }^{\beta}+\nabla^{\alpha} \partial^{\beta} \phi \tilde{R}_{\beta(\mu \nu) \alpha}\right], \\
& T_{\mu \nu}^{\lambda}=\lambda\left\{\begin{array}{l}
g_{\mu \nu}\left[(\square \phi)^{2}-\partial^{\alpha} \phi \partial_{\beta} \phi G_{\alpha \beta}+\partial^{\alpha} \phi \nabla_{\alpha} \square \phi-\partial^{\alpha} \phi \nabla^{\beta} \nabla_{\beta} \partial_{\alpha} \phi-\nabla_{\alpha} \partial^{\beta} \phi \nabla^{\alpha} \partial_{\beta} \phi\right] \\
\quad+2 \partial_{\mu} \phi \partial^{\alpha} \phi G_{\nu \alpha}+\partial_{\mu} \phi \partial_{\nu} \phi R+2 \nabla^{\alpha} \partial_{\mu} \phi \nabla_{\alpha} \partial_{\nu} \phi-2 \square \phi \nabla_{\nu} \partial_{\mu} \phi-\square \phi R_{\mu \nu} \\
\left.\quad+2 \partial^{\alpha} \phi\left(\nabla_{\mu} \nabla_{\alpha} \partial_{\nu} \phi+\nabla_{\nu} \nabla_{\alpha} \partial_{\mu} \phi+G_{\mu \alpha} \partial_{\nu} \phi-\nabla_{\alpha} \nabla_{\nu} \partial_{\mu} \phi\right)\right\} .
\end{array}\right.
\end{aligned}
$$

In the next subsection, we explain why the gauge and Chern-Simons couplings play an essential role in allowing axion hair on charged and rotating black holes.

\subsubsection{Long range axion hair and black holes}

Cosmological considerations motivate the investigation of light axion fields with Compton wavelengths well larger than the typical sizes of astrophysical black holes. In this context, the axion field can be considered as massless; it might acquire a long-range profile, affecting spherically symmetric configurations. But can a regular, asymptotically flat black hole with extended axion hair exist? There is a long list of no-hair theorems to overcome, starting from Bekenstein results [285, 2866], which forbid configurations with a long-range (pseudo)scalar $\phi$; no-hair arguments are particularly powerful in the case of shift-symmetric Lagrangians [288] like ours (see e.g. [154, 158, 164] for reviews). It is then interesting, both theoretically and phenomenologically, to assess whether long-range axion hair exist around black holes. The no-hair theorem discussed in [288] makes use of the properties of the conserved current $J^{\mu}$ associated with the shift symmetry: in fact, [288] shows that 
- for systems that do not contain linear terms in $\phi$ - regularity of the current at the horizon constrains a trivial radial profile for $\phi$. On the other hand, in the context of Horndeski theories, a way out has been found in [156] by noticing that the quintic Horndeski Lagrangian contains contributions linear in $\phi$ (coupling the field with the Gauss-Bonnet combination), hence evading the theorem and allowing for black hole hair in asymptotically flat configurations (exact solutions in related systems were found in $[\underline{\underline{\delta}}]]$ ).

In our shift-symmetric context described by action (4.2), we do as well have linear couplings of the axion field with gauge and gravitational Chern-Simons terms ${ }^{\text {m }}$, thus we could in principle find black holes with long-range axion hair. The aim of this work is indeed to demonstrate that these couplings offer new opportunities to find regular, asymptotically flat black hole solutions with long-range pseudoscalar fields. Examples of black hole solutions with axion hair, with and without charge and spin, have indeed been determined in the past [14.9, 150, 15.5, 28.9, 20.97[300], and the subject has been particularly developed in the context of ChernSimons gravity [15] (see e.g. [83] and the review [106]).

In our work, we further generalise these solutions at the light of these considerations, and extend them to the case of further derivative couplings of the axion with gravity, described by Lagrangian (A.T), and by considering the simultaneous presence of $g_{F}, g_{R}$ defined as in equations (4.7). The presence of Chern-Simons terms leads to sources for the axion field which are non-vanishing at spatial infinity, inevitably inducing long-range axion profiles around black holes. In particular, the $F \tilde{F}$ coupling provides a source for spherically symmetric dyonic black holes (with electric $(Q)$ and magnetic $(P)$ charges), since asymptotically far from the black hole it acquires the following profile:

$$
F \tilde{F} \sim \frac{P Q}{r^{4}}
$$

On the other hand, the gravitational $R \tilde{R}$ coupling sources an axion radial profile when the black hole geometry is axisymmetric, for example when it is rotating with rotation parameter $a=J / M,(J$ being the angular momentum and $M$ the

\footnotetext{
${ }^{1}$ Shift symmetry is ensured by the fact that $F \tilde{F}$ and $R \tilde{R}$ are by themselves total derivatives.
} 
black hole mass). In the uncharged case, and in the small rotation limit $(a \ll 1)$, one finds

$$
R \tilde{R} \sim \frac{a M^{2}}{r^{7}} \cos \theta
$$

Thus, the resulting axion field induced by the sources (4.17) and (4.18) backreacts on the geometry and affects the physical properties of the black hole at the position of the horizon.

In the next sections, we initially study charged spherically symmetric black hole configurations for this system; then we generalise to the study of slowly rotating charged solutions, and at the end we discuss phenomenological consequences of our results.

\subsection{Spherically symmetric configurations}

In this section, we discuss asymptotically flat, spherically symmetric configurations with axion hair around dyonic black holes, characterised by both magnetic and electric charges associated with the $U(1)$ gauge field $A_{\mu}$. This gauge field can be associated with some gauge symmetry beyond the Standard Model - for example associated with dark gauge bosons for dark matter interactions [6, 301, 302], or some additional gauge group motivated by string theory - or with standard electromagnetism: our discussion applies to both cases ${ }^{m}$. The Chern-Simons gauge coupling $F \tilde{F}$ sources an axion profile that scales as $1 / r$ asymptotically far from the object (while the gravitational Chern-Simons term $R \tilde{R}$ vanishes for spherically symmetric solutions). Its backreaction on the geometry and the gauge field can be analytically computed in a perturbative expansion in the dimensionless coupling constants $g_{F}$ and $\lambda$, appearing in action (4.J2) and scalar Lagrangian (4.T). We work at leading order in a perturbative expansion on these parameters to maintain our expressions relatively simple, and since interesting physical phenomena

\footnotetext{
${ }^{1}$ The configurations we consider differ from string theory configurations found in dilatonMaxwell-gravity systems and dilaton-axion-Maxwell-gravity systems (see e.g. respectively [311:3], [304] and the comprehensive review [30.5]), since in our case we do not have any scalar dilaton in our system, but only a pseudoscalar axion.
} 
already appear at leading order in such expansion. For completeness, in subsection 4.3 .2 we also discuss an exact solution for a specific, potentially large value of the coupling constant $\lambda$. The axion profile affects the electric field, but not the magnetic one. Our solution generalises the spherically symmetric configurations studied by Campbell et al [150, [28.9] and by Lee and Weinberg [14.9] (see also [2.97]) by including non-minimal couplings between axion and metric, described by the Horndeski coupling $\lambda$ in the Lagrangian density (4.T). As we will discuss, the properties of our configurations are different from other black hole solutions in theories with non-minimal couplings with gravity.

\section{The axion profile}

In a perturbative expansion of both $g_{F}$ and $\lambda$ parameters, from the equations of motion $(5.8,5.9,5.9)$ we observe that at leading order in the $g_{F}, \lambda$ couplings only the axion field's equation of motion gets a contribution. In fact, if we compare (5.9) with (5.8) and (5.9), we notice that a leading order contribution at the axion field implies a higher order contribution in both the metric and the gauge field. Therefore, we can initially solve the scalar field equation (5.9) at leading order in $g_{F}$ and $\lambda$ on a order zero background, and subsequently using the scalar field solution to solve the higher order metric and gauge field equations of motion. If we consider an electromagnetic field with vector potential $A_{\mu}$, the static, asymptotically flat, charged and spherically symmetric solution which satisfies the Einstein-Maxwell equations is the dyonic Reissner-Nordström $(\mathrm{RN})$ metric

$d s^{2}=\left(-1+\frac{2 M}{r}-\frac{P^{2}+Q^{2}}{r^{2}}\right) d t^{2}+\left(1-\frac{2 M}{r}+\frac{P^{2}+Q^{2}}{r^{2}}\right)^{-1} d r^{2}+r^{2} d \theta^{2}+r^{2} \sin ^{2} \theta d \varphi^{2}$

where $Q$ and $P$ are the electric and the magnetic charge ${ }^{\varpi}$ respectively, and where $r_{ \pm}$correspond to the outer/inner horizons of the RN black hole:

$$
r_{ \pm}=M \pm \sqrt{M^{2}-\left(P^{2}+Q^{2}\right)}
$$

\footnotetext{
${ }^{1}$ As explained in the motivations, the inclusion of the magnetic charge comes from the full electromagnetic dualism, as predicted by string theory.
} 
The $U(1)$ gauge field associated with the Reissner-Nordström metric is

$$
A_{\mu}=\left\{ \pm \frac{Q}{r}, 0,0, \pm P \cos \theta\right\}
$$

where the $A_{0}$ component

$$
A_{0}= \pm \frac{Q}{r}
$$

is defined up to a constant in accordance with gauge invariance. Since the geometry is static, the gravity Chern-Simons term $\tilde{R}_{\alpha \beta \gamma \delta} R^{\alpha \beta \gamma \delta}$ is everywhere null, since the Riemann tensor is everywhere vanishing. Instead, the electromagnetic ChernSimons term $\tilde{F}_{\alpha \beta} F^{\alpha \beta}$ is not flat if both the electric and the magnetic charges are on, thus we have:

$$
\tilde{F}^{\alpha \beta} F_{\alpha \beta}=-g_{F} \frac{P Q}{r^{4}}, \quad \tilde{R}^{\alpha \beta \gamma \delta} R_{\alpha \beta \gamma \delta}=0 .
$$

Explicitly, the equation of motion for the axion $\phi(t, r, \theta, \varphi)$ at leading order in $g_{F}$ and $\lambda$ reads

$$
\begin{aligned}
& \frac{\Delta\left[2 \lambda\left(P^{2}+Q^{2}\right)+r^{4}\right]}{r^{6}} \frac{\partial^{2} \phi}{\partial r^{2}}+\left[4 \lambda\left(P^{2}+Q^{2}\right) \frac{\left(\Delta-M r+P^{2}+Q^{2}\right)}{r^{7}}+\frac{2(r-M)}{r^{2}}\right] \frac{\partial \phi}{\partial r} \\
& +\frac{1}{\sin \theta} \frac{\partial}{\partial \theta}\left(\sin \theta \frac{\partial \phi}{\partial \theta}\right) \frac{2 \lambda\left(P^{2}+Q^{2}\right)+r^{4}}{r^{6}}+\frac{g_{F} P Q}{r^{4}}=0,
\end{aligned}
$$

where as in the RN case we have $\Delta=r^{2}-2 M r+P^{2}+Q^{2}$. Since the source term on the RHS of equation (4.24) does not depend on the coordinates $(t, \theta, \varphi)$, we expect and therefore assume that any supported scalar field configuration has to be static and spherically symmetric and it only depends on the radial coordinate:

$$
\phi(t, r, \theta, \varphi)=\phi(r)
$$

With this assumption, the axion equation simply reduces to

$$
\begin{aligned}
& {\left[\frac{4 \lambda\left(P^{2}+Q^{2}\right)\left(\Delta-M r+P^{2}+Q^{2}\right)}{r^{7}}+\frac{2(r-M)}{r^{2}}\right] \phi^{\prime}} \\
& +\frac{\Delta}{r^{2}}\left[1-\frac{2 \lambda\left(P^{2}+Q^{2}\right)}{r^{6}}\right] \phi^{\prime \prime}=-\frac{g_{F} P Q}{r^{4}} .
\end{aligned}
$$


Integrating the above equation, we find the radial scalar field solution up to two integration constant $\left(C_{1}, C_{2}\right)$ :

$$
\phi\left(r ; C_{1}, C_{2}\right) .
$$

Since we are interested in quasi-bounded scalar field configurations, i.e. solutions which are regular on the outer horizon and decay to zero at spatial infinity, we impose quasi-bounded boundary conditions fixing both $C_{1}$ and $C_{2}$ integration constants. At leading order in an expansion in both $g_{F}$ and $\lambda$, the unique solution for the scalar field's equation of motion that is regular at the black hole's horizon and decays at spatial infinity is

$$
\phi(r)=-g_{F} P Q\left\{\frac{\log \left(1-\frac{r_{-}}{r}\right)}{r_{-} r_{+}}-\frac{\lambda}{r_{-}^{4}}\left[\frac{2 r_{-}}{r}+\frac{r_{-}^{2}}{r^{2}}+\frac{2 r_{-}^{3}}{3 r^{3}}+\frac{r_{-}^{4}}{2 r^{4}}+2 \log \left(1-\frac{r_{-}}{r}\right)\right]\right\},
$$

where we could have added to this expression a constant $\phi_{0}$, which however has no physical consequences due to shift-symmetry.

The axion profile (4.28) describes a secondary hair, since there is no independent pseudoscalar charge but it is fully controlled by the black hole's charges $(M, P, Q)$; on the other hand, as we will see, the axion affects some properties of the black hole's horizon. Interestingly, we notice that the axion profile is regular at the position $r_{+}$of the outer horizon, while it diverges at the inner horizon $r_{-}$(more on this later). Expanding for large values of $r$, one finds ${ }^{\text {m }}$

$$
\phi \sim\left(\frac{g_{F} P Q}{r_{+}}\right) \frac{1}{r}+\mathcal{O}\left(\frac{1}{r^{2}}\right)
$$

Interestingly, we find that the pseudoscalar axion profile decays as $1 / r$, thus charged black holes with gauge couplings $\phi F \tilde{F}$ support monopolar axion configurations. This is a difference with respect to what happens with gravitational Chern-Simons couplings $\phi R \tilde{R}$, as we will discuss in section 4.4 , where the axion radial profile decays as $1 / r^{2}$ [15.5]. Such axion profile has newsworthy consequences for the physics of the horizon and for the phenomenology of the system. In fact,

\footnotetext{
${ }^{1}$ It is straightforward to verify that this asymptotic, large $r$ profile for the scalar field remains valid also beyond a leading order expansion in the coupling constants $\left(g_{F}, \lambda\right)$. We will use this fact in section 4.5 .1 .
} 
although the axion scales with the radial coordinate as $1 / r$, it can more easily circumvent fifth force constraints [245, 306], since being a pseudoscalar it does not directly couple to the trace of stress-energy tensor. Moreover, one can check that the corrections weighted by $\lambda$, associated with derivative couplings with the metric, appear in the axion profile at order $1 / r^{4}$ : this is probably a manifestation of Vainshtein screening in this context (see e.g. [5.5] for a review).

\section{Gauge field solution}

The vector potential associated to this configuration has both electric and magnetic components

$$
A_{\mu}=\left\{A_{t}(r), 0,0, A_{\varphi}(\theta)\right\}
$$

where, at leading order in a $\left(g_{F}, \lambda\right)$ expansion, we find the solution

$$
\begin{aligned}
A_{t}(r)= & -\frac{Q}{r}+\frac{g_{F}^{2} P^{2} Q}{r}[a(r)+\lambda b(r)], \\
a(r)= & \frac{r_{-}\left(r_{-} r_{+}-2 Q^{2}\right)+\left[Q^{2}\left(r_{-}-2 r\right)+r_{+} r_{-}\left(r-r_{-}\right)\right] \log \left(1-\frac{r_{-}}{r}\right)}{r_{-}^{3} r_{+}^{2}} \\
b(r)= & \frac{1}{30 r^{5} r_{-}^{2}}\left\{r _ { - } r _ { + } \left[60 r^{4}\left(r_{-}+4 r_{+}\right)+30 r^{3} r_{-}\left(r_{-}-4 r_{+}\right)+20 r^{2} r_{-}^{2}\left(r_{-}-2 r_{+}\right)\right.\right. \\
& \left.+5 r r_{-}^{3}\left(3 r_{-}-4 r_{+}\right)-12 r_{-}^{4} r_{+}\right]-5 P^{2}\left[12 r^{4}\left(r_{-}+5 r_{+}\right)+6 r^{3} r_{-}\left(r_{-}-5 r_{+}\right)\right. \\
& \left.+2 r^{2} r_{-}^{2}\left(2 r_{-}-5 r_{+}\right)+r r_{-}^{3}\left(3 r_{-}-5 r_{+}\right)-3 r_{-}^{4} r_{+}\right] \\
& \left.-60 \frac{r^{4}}{r_{-}} \log \left(1-\frac{r_{-}}{r}\right)\left[Q^{2}\left(5 r_{-} r_{+}-r r_{-}-5 r_{+} r\right)+r_{-} r_{+}\left(r r_{+}-r_{-} r_{+}\right)\right]\right\} \\
A_{\varphi}(\theta)= & -P \cos \theta
\end{aligned}
$$

with $r_{ \pm}$being the outer and inner horizon's radii, respectively. Such vector configuration corresponds to a magnetic monopole configuration with magnetic charge $P$, additionally charged under an electric field $E_{r}=\partial_{r} A_{t}(r)$ whose profile is mod- 
ulated by the axion field, and by the presence of the magnetic charge $P$. This fact resembles, in curved space-times, a phenomenon called Witten effect [307, 308], where an axion configuration switches on an electric field in a magnetic monopole background in the presence of a Chern-Simons coupling $\phi F \tilde{F}$.

\section{Metric solution}

Given the scalar hair solution (4.28), we now calculate the background metric's corrections due to the backreaction of the scalar field.

At leading order in a $\left(g_{F}, \lambda\right)$ expansion, the metric line element reads as follows:

$$
d s^{2}=-F(r) d t^{2}+\frac{d r^{2}}{h(r) F(r)}+r^{2} d \theta^{2}+r^{2} \sin ^{2} \theta d \varphi^{2}
$$

with

$$
\begin{aligned}
F(r)= & \left(1-\frac{r_{-}}{r}\right)\left(1-\frac{r_{+}}{r}\right)+g_{F}^{2}\left(\frac{P Q}{r_{-} r_{+}}\right)^{2}\left\{-\frac{2 r_{-}}{r}+\frac{r_{+} r_{-}}{r^{2}}-\frac{2 r-r_{+}-r_{-}}{r} \log \left(1-\frac{r_{-}}{r}\right)\right. \\
& +\frac{\lambda}{30 r_{-}^{4} r_{+}}\left[\frac{600}{r}-\frac{60\left(4 r_{-}+9 r_{+}\right)}{r^{2}}-\frac{70 r_{-}\left(r_{-}-3 r_{+}\right)}{r^{3}}-\frac{30 r_{-}^{2}\left(r_{-}-2 r_{+}\right)}{r^{4}}\right. \\
& \left.\left.-\frac{5 r_{-}^{3}\left(3 r_{-}-5 r_{+}\right)}{r^{5}}+\frac{12 r_{-}^{4} r_{+}}{r^{6}}+\frac{60\left(10 r^{2}-9 r\left(r_{-}+r_{+}\right)+8 r_{-} r_{+}\right) \log \left(1-\frac{r_{-}}{r}\right)}{r^{2} r_{-}}\right]\right\}
\end{aligned}
$$

and

$$
\begin{aligned}
h(r)= & +1+\frac{2 g_{F}^{2} P^{2} Q^{2}}{r_{-}^{2} r_{+}^{2}}\left\{\frac{r_{-}}{r-r_{-}}+\log \left(1-\frac{r_{-}}{r}\right)-\lambda \frac{10 r_{+}}{r_{-}^{2}\left(r-r_{-}\right)}\left[1-\frac{r_{-}}{2 r}-\frac{r_{-}^{2}}{6 r^{2}}\right.\right. \\
& \left.\left.-\frac{r_{-}^{3}\left(5 r_{+}-12 r_{-}\right)}{60 r_{+} r^{3}}-\frac{r_{-}^{4}}{4 r^{4}}-\left(1-\frac{r}{r_{-}}\right) \log \left(1-\frac{r_{-}}{r}\right)\right]\right\} .
\end{aligned}
$$

Setting $g_{F}=0$, one finds the standard Reissner-Nordström solution with magnetic and electric charges. Instead, setting only $\lambda=0$ one finds the solution of [149]. The axion profile backreacts on the metric, with corrections starting only at order $1 / r^{3}$ in a $1 / r$ expansion. Interestingly, the axion profile changes the position of the outer horizon of the charged black hole: at leading order in an expansion in 
the dimensionless couplings $\left(g_{F}, \lambda\right)$, the position of the outer horizon is

$$
r_{H}=r_{+}+g_{F}^{2} r_{S}
$$

where we have defined

$$
\begin{aligned}
r_{S}= & +\frac{P^{2} Q^{2}}{r_{+} r_{-}^{2}}\left[\log \left(1-\frac{r_{-}}{r_{+}}\right)+\frac{r_{-}}{r_{+}-r_{-}}\right. \\
& \left.+\lambda \frac{r_{-}\left(3 r_{-}^{4}+5 r_{-}^{3} r_{+}+10 r_{-}^{2} r_{+}^{2}+30 r_{-} r_{+}^{3}-60 r_{+}^{4}\right)-60 r_{+}^{4}\left(r_{+}-r_{-}\right) \log \left(1-\frac{r_{-}}{r_{+}}\right)}{30 r_{-}^{3} r_{+}^{3}\left(r_{+}-r_{-}\right)}\right] .
\end{aligned}
$$

The geometry is regular at the outer horizon, and we find (in our approximations scheme) that the presence of the axion hair increases the size of the outer horizon. Curvature invariants diverge at the position of the inner horizon $r_{-}$, hence the axion profile makes singular the inner horizon of the charged black hole. This fact was already found in [149, 28.9] for the case with minimal couplings with gravity, and it remains true also in our set-up with derivative couplings to gravity described by Lagrangian (4.1). So, as anticipated in subsection 4.2.2, Chern-Simons gauge couplings $\phi F \tilde{F}$ provide qualitatively new opportunities to find new black hole solutions with axion hair, also in the case of non-minimal couplings of the axion with gravity. Our analytic formulas - valid for small values of the coupling constants describe how the black hole geometry is affected by the long-range axion configurations.

To conclude this subsection, we qualitatively compare some aspects of our findings with some of the regular black hole solutions in Horndeski theories. In [156, 287], asymptotically flat, hairy solutions have been found in systems with a linear coupling between the scalar field and the Gauss-Bonnet combination (hence avoiding the no-hair theorem [288], see our section 4.2.2), weighted by a dimensionless quantity that we call $g_{G B}$. Moreover, [287] determines analytical solutions for the system in a perturbative expansion for small $g_{G B}$, finding that the geometry is affected only at next to leading order in such expansion, requiring a control of the theory up to next-to-leading level in the small parameter $g_{G B}$. Instead, in our case, differences with respect to the standard Reissner-Nordström configuration are already visible at leading order in our parameter expansion. Moreover, 
the works [30.9-3प] determine regular black hole solutions with non-minimal couplings to gravity like ours, but in order to avoid the no-hair theorem [288] the solutions are not asymptotically flat. A more comprehensive discussion of black holes in theories non-minimally coupled with gravity can be found in the review [312].

\subsubsection{A Smarr formula for our configurations}

Since we analytically determined - at leading order in a perturbative expansion in the parameters $\left(g_{F}, \lambda\right)$ - how the axion profile modifies the location of black hole's horizon, we can enquire whether classical black hole thermodynamics formulas remain valid in our system (see also [31.3] for a general discussion on black hole thermodynamics in theories containing non-minimal couplings with gravity and higher derivative interactions). The Smarr formula [17] is associated with the first law of thermodynamics, and relates the black hole entropy with conserved asymptotic charges. In the context of spherically symmetric geometries it reads:

$$
M=\frac{\kappa}{2 \pi} S+J \Omega_{H}+Q \Phi_{H}^{E}+P \Phi_{H}^{M}
$$

where $M$ is the ADM mass, $\kappa$ is the surface gravity, $S$ is the black hole's entropy, $J$ is the angular momentum, $\Omega_{H}$ is the black hole's angular velocity, $Q$ and $P$ are the electric and the magnetic charges respectively and $\Phi_{H}^{E, M}$ respectively are the electric and magnetic potentials evaluated at the horizon. As already discussed in the theory review in section $\square .2 .4$, the differential version of the Smarr formula (4.37) can be regarded as the first law of black hole thermodynamics, with $\kappa$ playing the same role of the temperature:

$$
T_{H}=\frac{\kappa}{2 \pi} \Rightarrow T_{H}=\frac{\partial E}{\partial S}=\frac{\partial M}{\partial S}
$$

Exploiting this analogy, we can relate the black hole entropy with the event horizon area through the relation

$$
S=\frac{A_{H}}{4},
$$


and hence we can rewrite the differential Smarr formula as

$$
d M=\frac{\kappa}{8 \pi} d A_{H}+\Omega_{H} d J+\Phi_{H}^{E} d Q+\Phi_{H}^{M} d P
$$

The event horizon area $A_{H}$ is given by the integral of the 2-dimensional induced volume element [2:3]

$$
A_{H}=\int \sqrt{\left|\Omega_{S^{2}}\right|} d \theta d \varphi
$$

with $\left|\Omega_{S^{2}}\right|$ being the determinant of the induced 2-dimensional metric on the horizon of the complete 4-dimensional metric $g_{\mu \nu}$.

The horizon's angular velocity is defined as

$$
\Omega_{H}=\left.\frac{d \varphi}{d t}\right|_{r_{+}}=\frac{a}{r_{+}^{2}+a^{2}}
$$

and the electric potential is given by

$$
\Phi_{H}^{E}=-A_{t}+\Omega_{H} A_{\varphi},
$$

where $A_{t}$ and $A_{\varphi}$ are respectively the $t$-component and the $\varphi$-component of the electromagnetic gauge vector $A_{\mu}$ in the Boyer-Lindquist coordinate system $(t, r, \theta, \varphi)$. For a static dyonic Reissner-Nordström black hole we have

$$
\Omega_{H}=0
$$

and the electric and the magnetic potential on the horizon are ([314], [315])

$$
\Phi_{H}^{E}=\frac{Q}{r_{+}}, \quad \Phi_{H}^{M}=\frac{P}{r_{+}},
$$

and the differential Smarr formula (4.37) reads:

$$
d M=\frac{\kappa}{8 \pi} d A_{H}+\Phi_{H}^{E} d Q+\Phi_{H}^{M} d P
$$

with surface gravity and event horizon area given by

$$
\kappa=\frac{r_{+}-r_{-}}{2 r_{+}^{2}}, \quad A_{H}=4 \pi r_{+}^{2}
$$


For our configurations (4.28), at leading order in $\left(g_{F}, \lambda\right)$ we find:

$$
\begin{gathered}
\kappa=+\frac{r_{+}-r_{-}}{2 r_{+}^{2}}-\frac{g_{F}^{2} P^{2} Q^{2}}{r_{-}^{2} r_{+}^{4}}\left[\frac{r_{-}\left(r_{+}-2 r_{-}\right)+\left(r_{+}-r_{-}\right)^{2} \log \left(1-\frac{r_{-}}{r_{+}}\right)}{r_{+}-r_{-}}-\lambda k_{\lambda}\right] \\
k_{\lambda}=\frac{\left(r_{-}^{5}-2 r_{-}^{4} r_{+}-5 r_{-}^{3} r_{+}^{2}-20 r_{-}^{2} r_{+}^{3}+90 r_{-} r_{+}^{4}-60 r_{+}^{5}\right)-60 r_{+}^{4} r_{-}\left(1-\frac{r_{+}}{r_{-}}\right)^{2} \log \left(1-\frac{r_{-}}{r_{+}}\right)}{10 r_{-}^{2} r_{+}^{3}\left(r_{+}-r_{-}\right)} \\
A_{H}=4 \pi\left(r_{+}^{2}+2 g_{F}^{2} r_{S} r_{+}\right) \\
\Omega_{H}=0 \\
\Phi_{H}^{E}=-A_{t}(r) \\
\Phi_{H}^{M}=\Phi_{H}^{E}(P \leftrightarrow Q)
\end{gathered}
$$

where $A_{t}$ is given in equation (4.30), $r_{S}$ in (4.36), and the magnetic potential can be computed using the procedure explained in [22], which in our case is simply related with the electric potential interchanging $P$ with $Q$. Substituting these quantities in (4.37), we find that the Smarr formula is satisfied for our black hole solutions: hence the first law of thermodynamics applies also to dyonic black holes with axion fields non-minimally coupled with gravity.

In [3/6], it has been shown that the scalar field contribution to the black hole thermodynamics is described by the additional term in the Smarr formula

$$
\left(\frac{\partial M}{\partial \phi}\right) d \phi
$$

where

$$
\left(\frac{\partial M}{\partial \phi}\right)=\Sigma
$$

and with $\Sigma$ being the scalar charge at infinity, namely

$$
\phi=\frac{\Sigma}{r}+\mathcal{O}\left(\frac{1}{r^{2}}\right)
$$

Surprisingly, for our black hole configuration the Smarr formula is satisfied without the extra term coming from the scalar charge: a possible reason is that the axion 
profile does not modify the asymptotic conserved charges of our configuration $(M, P, Q)$, since it only affects metric and gauge fields with higher order corrections in a $1 / r$ expansion. In fact, the asymptotic black hole's mass and charges do not receive any contribution from the axion, which consequently does not affect the overall energetic balance controlled by the Smarr formula.

\subsubsection{An exact solution for large values of $\lambda$}

In the previous section, we discussed a solution for a spherically symmetric dyonic black hole configuration at leading order in a perturbative expansion in the coupling constant $\lambda$, affecting derivative couplings of the axion to the curvature, introduced in equation (4.T). Solutions for arbitrary values of $\lambda$ can be found in general, but their expressions are very cumbersome. On the other hand, for special values of this parameter, their expression simplifies. For example, with the particular choice

$$
\lambda=-\frac{r_{-}^{3}}{2 r_{+}}
$$

the solution for the axion configuration is relatively simple, and reads

$$
\phi(r)=\frac{g_{F} P Q}{8 r_{-} r_{+}}\left[\frac{2 r_{-}}{r-r_{-}}+\log \left(r^{2}+r_{-}^{2}\right)+\log \left(r+r_{-}\right)-3 \log \left(r-r_{-}\right)+2 \tan ^{-1}\left(\frac{r_{-}}{r}\right)\right] .
$$

Notice that the choice (4.53) allows for tuning large values of $\lambda$, by choosing $r_{+}$ and $r_{-}$appropriately. Assuming a generic metric line element

$$
d s^{2}=-F(r) d t^{2}+\frac{d r^{2}}{h(r) F(r)}+r^{2} d \theta^{2}+r^{2} \sin ^{2} \theta d \varphi^{2}
$$

choosing $\lambda$ as in eq (4.5.3), at leading order in the coupling $g_{F}$ we find

$$
\begin{aligned}
F(r)= & \left(1-\frac{r_{+}}{r}\right)\left(1-\frac{r_{-}}{r}\right)+\frac{g_{F}^{2} P^{2} Q^{2}}{4 r_{-}^{2} r_{+}^{2}} f(r) \\
f(r)= & \frac{r_{-}\left(4 r_{+}-3 r\right)}{r^{2}}+\frac{\left(2 r-r_{-}-3 r_{+}\right)}{r} \tan ^{-1}\left(\frac{r_{-}}{r}\right)+\frac{\left(r^{2}-2 r r_{+}-r_{-} r_{+}\right)}{2 r^{2}} \log \left(r+r_{-}\right) \\
& -\frac{\left(r^{2}+2 r r_{-}-5 r_{-} r_{+}\right)}{2 r^{2}} \log \left(r-r_{-}\right)+\frac{\left(r r_{-}+r r_{+}-2 r_{-} r_{+}\right)}{2 r^{2}} \log \left(r^{2}+r_{-}^{2}\right)
\end{aligned}
$$




$$
\begin{aligned}
h(r)= & +1-\frac{g_{F}^{2} P^{2} Q^{2}}{2 r_{+}^{2}}\left[\frac{3 r^{8} r_{+}+r^{7} r_{-} r_{+}+r^{6} r_{-}^{2} r_{+}+r^{5} r_{-}^{3}\left(8 r_{-}+r_{+}\right)-13 r^{4} r_{-}^{4} r_{+}}{2 r_{-} r_{+}\left(r-r_{-}\right)^{3}\left(r+r_{-}\right)^{2}\left(r^{2}+r_{-}^{2}\right)^{2}}\right. \\
& \left.+\frac{-r^{3} r_{-}^{5} r_{+}-r^{2} r_{-}^{6} r_{+}-r r_{-}^{7} r_{+}+2 r_{-}^{8} r_{+}}{2 r_{-} r_{+}\left(r-r_{-}\right)^{3}\left(r+r_{-}\right)^{2}\left(r^{2}+r_{-}^{2}\right)^{2}}+\frac{2 \tan ^{-1}\left(\frac{r_{-}}{r}\right)+\tanh ^{-1}\left(\frac{r_{-}}{r}\right)}{r_{-}^{2}}\right] .
\end{aligned}
$$

As in the small coupling approximation $\lambda \ll 1$ (see section 42.2 ), the ReissnerNordström case is recovered setting $g_{F}=0$, while metric corrections due to axion's backreaction begin to appear only at order $1 / r^{3}$ in a $1 / r$ expansion. At leading order in a $g_{F}$ expansion, the outer horizon is located at

$$
r_{H}=r_{+}+g_{F}^{2} r_{S}
$$

with

$$
\begin{aligned}
r_{S}=\frac{P^{2} Q^{2}}{8 r_{-}^{2} r_{+}}[ & -\frac{2 r_{-}}{\left(r_{+}-r_{-}\right)}-\log \left(r_{-}^{2}+r_{+}^{2}\right)+\frac{\left(r_{+}-3 r_{-}\right)}{\left(r_{+}-r_{-}\right)} \log \left(r_{+}-r_{-}\right) \\
& \left.+\frac{\left(r_{-}+r_{+}\right)}{\left(r_{+}-r_{-}\right)} \log \left(r_{-}+r_{+}\right)+\frac{2\left(r_{-}+r_{+}\right)}{\left(r_{+}-r_{-}\right)} \tan ^{-1}\left(\frac{r_{-}}{r_{+}}\right)\right] .
\end{aligned}
$$

With the particular choice (4.5.3), compared with the Reissner-Nordström case the axion's backreaction increases the size of the outer horizon. It would be interesting to understand if there is any choice of the coupling $\lambda$ which could lead to a reduction of the size of the outer horizon, but we leave it to future works. The geometry is regular on the outer horizon $r_{H}$ and everywhere outside it, while there is a singularity located on the inner horizon $r_{-}$, which cannot be removed by any choice of the coupling parameter $\lambda$. Moreover, the axion's backreaction also modifies the gauge potential

$$
A_{\mu}=\left\{A_{t}(r), 0,0,-P \cos \theta\right\},
$$

and at leading order in a $g_{F}$ expansion we find 


$$
\begin{aligned}
A_{t}(r)=-\frac{Q}{r}+g_{F}^{2} Q\{ & {\left[\frac{r^{5}\left(3 r_{-}+r_{+}\right)+\left(r_{+}-r_{-}\right)\left(r^{4} r_{-}+r^{3} r_{-}^{2}+r^{2} r_{-}^{3}\right)+4 r r_{-}^{5}-8 r_{-}^{5} r_{+}}{4 r r_{+}\left(r-r_{-}\right)^{2}\left(r+r_{-}\right)\left(r^{2}+r_{-}^{2}\right)}\right.} \\
& -\frac{\left(r_{-}-5 r_{+}\right) \log \left(r^{2}+r_{-}^{2}\right)}{4 r_{-} r_{+}}+\frac{\left(5 r r_{-}-13 r r_{+}+4 r_{-} r_{+}\right) \log \left(r-r_{-}\right)}{8 r r_{-} r_{+}} \\
& \left.-\frac{\left(r r_{-}+7 r r_{+}+4 r_{-} r_{+}\right) \log \left(r+r_{-}\right)}{8 r r_{-} r_{+}}+\frac{\tan ^{-1}\left(\frac{r}{r_{-}}\right)}{r_{-}}\right] \frac{P^{2} Q^{2}}{8 r_{-}^{2} r_{+}^{2}} \\
& +\frac{P^{2}}{4 r_{-}^{2} r_{+}}\left[\frac{\left(r_{-}-r\right) \log \left(r^{2}+r_{-}^{2}\right)}{2 r}+\frac{\left(r-3 r_{-}\right) \log \left(r-r_{-}\right)}{2 r}\right. \\
& \left.\left.+\frac{\left(r+r_{-}\right) \log \left(r+r_{-}\right)}{2 r}-\frac{r \tan ^{-1}\left(\frac{r}{r_{-}}\right)+r_{-}}{r}+\frac{P^{2}}{r_{+}} \frac{\tan ^{-1}\left(\frac{r_{-}}{r}\right)}{r}\right]\right\} .
\end{aligned}
$$

As it happens for the perturbative solution (4.3) in the previous section, the magnetic potential is the same as in Einstein-Maxwell theory, while the electric potential is modified by the presence of the axion field.

\subsection{Slowly rotating charged configurations}

When considering rotating black holes, besides the gauge Chern-Simons coupling, also the gravitational Chern-Simons coupling $\phi R \tilde{R}$ acquires a non-zero value - see equation (4.18) - and it can source a non-trivial axion profile. This fact is related with the well-developed topic of rotating black hole solutions in Chern-Simons gravity, see e.g. [83, $317-323]$ and the review [16]. In this section, we investigate for the first time how the simultaneous presence of gauge and gravitational ChernSimons couplings affects the geometry of rotating charged black holes with a longrange axion hair. To stress the role of the gravitational Chern-Simons couplings, we consider only black holes with a magnetic monopole charge (no electric charge), and we work in the limit of small rotation, $a=J / M \ll 1$. For simplicity, we also turn off the non-minimal coupling with gravity, setting $\lambda=0$ in Lagrangian (4.D). Notice that this situation is different from Wald configuration of a black hole immersed in an external magnetic field [324], since in our case the black hole sources the magnetic field and it is therefore charged. 
The action describing the system we are considering reads

$$
S=\int d^{4} x \sqrt{g}\left[\frac{R}{4}-\frac{1}{4} F_{\mu \nu} F^{\mu \nu}-\frac{1}{2} \partial_{\mu} \phi \partial^{\mu} \phi-\frac{g_{F}}{8} \phi \tilde{F}^{\mu \nu} F_{\mu \nu}+\frac{g_{R}}{8} \phi \tilde{R}^{\mu \nu \alpha \beta} R_{\mu \nu \alpha \beta}\right]
$$

where $g_{F}$ and $g_{R}$ denote the coupling constants of the gauge and the gravity ChernSimons couplings, respectively.

For analytical solutions, we work in a perturbative expansion scheme of the parameters $\left(a, g_{F}, g_{R}\right)$; as explained in the spherically symmetric case, the scalar field backreaction on the background metric and gauge field appears at higher orders than the leading one, namely the leading order of the scalar field solution. Therefore, as we did for the spherical case, we can solve the leading order scalar field equation on a fixed "lower order" background. However, in this case we cannot use the order zero (on both the rotation parameter and couplings) background; in fact, since the Chern-Simons source term $\phi R \tilde{R}$ acquires a non-zero value only if the rotation parameter $a$ is not vanishing, the scalar field solution has leading order $\left(a, g_{i}\right)$. Thus, to solve the scalar field's equation of motion we have to consider the $(a, 0)$ order background metric and gauge field, and then using the leading order scalar field solution to compute higher orders corrections at both the metric and the gauge field. So, neglecting terms of order $\mathcal{O}\left(a^{2}\right)$ or greater, the background metric is the linearised magnetic Kerr-Newman one:

$$
\begin{aligned}
d s^{2}= & -\left(1-\frac{2 M}{r}+\frac{P^{2}}{r^{2}}\right) d t^{2}+\left(1-\frac{2 M}{r}+\frac{P^{2}}{r^{2}}\right)^{-1} d r^{2}+r^{2} d \theta^{2}+r^{2} \sin ^{2} \theta d \varphi^{2} \\
& +a \sin ^{2} \theta\left(-\frac{2 M}{r}+\frac{P^{2}}{r^{2}}\right) d t d \varphi+\mathcal{O}\left(a^{2}\right)
\end{aligned}
$$

and the gauge vector is

$$
A_{\mu}=\left\{\frac{a P \cos \theta}{r^{2}}, 0,0, \frac{-P r \cos \theta}{r}\right\}+\mathcal{O}\left(a^{2}\right)
$$


At leading order, the gravity Chern-Simons source term and the electromagnetic one are

$$
\begin{aligned}
& \tilde{R}^{\mu \nu \alpha \beta} R_{\mu \nu \alpha \beta}=-\frac{24 a\left[2 P^{2}-3 M r\right]\left(P^{2}-M r\right) \cos \theta}{r^{9}}+\mathcal{O}\left(a^{2}\right) \\
& \tilde{F}^{\mu \nu} F_{\mu \nu}=\frac{2 a P^{2} \cos \theta}{r^{5}}+\mathcal{O}\left(a^{2}\right) .
\end{aligned}
$$

Solving the Klein-Gordon equation

$$
\square \phi=g_{F} \tilde{F}^{\mu \nu} F_{\mu \nu}-g_{R} \tilde{R}^{\mu \nu \alpha \beta} R_{\mu \nu \alpha \beta}
$$

on the background (4.6.3) and (4.5.5), after imposing a quasi-bounded behaviour of the solution by fixing the integration constants, we find that the associated profile for the pseudoscalar axion field has a dipolar structure (i.e. it decays as $1 / r^{2}$ ) and it results

$$
\begin{aligned}
\phi(r, \theta)= & a g_{F} \frac{P^{6}}{\left(r_{+} r_{-}\right)^{4}}\left[2(M-r) \log \left(\frac{r-r_{-}}{r}\right)+\frac{r_{+} r_{-}}{r}-2 r_{-}\right] \cos \theta \\
& -\frac{8 a g_{R}}{5}\left\{\frac{10 M^{4}-21 M^{2}\left(r_{+} r_{-}\right)+6\left(r_{+} r_{-}\right)^{2}}{2 r\left(r_{+} r_{-}\right)^{3}}-\frac{29 M\left(r_{+} r_{-}\right)-15 M^{3}}{12 r^{2}\left(r_{+} r_{-}\right)^{2}}\right. \\
& -\frac{18\left(r_{+} r_{-}\right)-5 M^{2}}{12 r^{3}\left(r_{+} r_{-}\right)}-\frac{5 M}{4 r^{4}}+\frac{\left(r_{+} r_{-}\right)}{r^{5}} \\
& \left.+\frac{10 M^{4}-21 M^{2}\left(r_{+} r_{-}\right)+6\left(r_{+} r_{-}\right)^{2}}{\left(r_{+} r_{-}\right)^{4}}\left[(M-r) \log \left(\frac{r-r_{-}}{r}\right)-r_{-}\right]\right\} \cos \theta .
\end{aligned}
$$

Interestingly, it includes contributions from both the gauge and gravitational Chern-Simons terms, weighted by the (small) rotation parameter $a$. We can now use the solution (4.67) to find the backreaction contributions to the metric and the gauge field. The Ansatz we adopt for the metric is

$$
d s^{2}=-F(r) d t^{2}+\frac{d r^{2}}{F(r)}+r^{2} d \theta^{2}+r^{2} \sin ^{2} \theta d \varphi^{2}+2 a w(r) r^{2} \sin ^{2} \theta d t d \varphi,
$$


where the quantity $w(r)$ in the off-diagonal component controls the effects of black hole's rotation, and the gauge vector has the structure

$$
A_{\mu}=\left\{A_{t}(r, \theta) 0,0, A_{\varphi}(r, \theta)\right\}
$$

At leading order in the coupling constants $g_{F}$ and $g_{R}$, we find the solution

$$
\begin{aligned}
F(r)= & 1-\frac{2 M}{r}+\frac{P^{2}}{r^{2}} \\
w(r)= & -\frac{2 M}{r^{3}}+\frac{P^{2}}{r^{4}}-\frac{g_{F} g_{R}}{2} P^{2}\left(\frac{3}{8 M^{2} r^{4}}+\frac{1}{5 M r^{5}}+\frac{21}{10 r^{6}}\right)+g_{R}^{2}\left[\frac{5}{2 r^{6}}+\frac{30 M}{7 r^{7}}+\frac{27 M^{2}}{4 r^{8}}\right. \\
& \left.-P^{2}\left(\frac{3}{16 M^{4} r^{4}}+\frac{9}{40 M^{3} r^{5}}+\frac{3}{10 M^{2} r^{6}}+\frac{41 M}{4 r^{9}}+\frac{18}{7 M r^{7}}+\frac{21}{4 r^{8}}\right)\right]
\end{aligned}
$$

and the gauge components

$$
\begin{aligned}
& A_{t}(r, \theta)=\frac{a P \cos \theta}{r^{2}}\left[1+g_{F} g_{R}\left(-\frac{3}{16 M^{2}}+\frac{1}{8 M r}+\frac{11}{40 r^{2}}+\frac{9 M}{20 r^{3}}\right)\right. \\
& \left.-g_{R}^{2}\left(\frac{3}{16 M^{4}}+\frac{3}{16 M^{3} r}+\frac{9}{40 M^{2} r^{2}}+\frac{3}{10 M r^{3}}+\frac{1}{4 r^{4}}+\frac{9 M}{56 r^{5}}\right)\right], \\
& A_{\varphi}(r, \theta)=-P \cos \theta \text {. }
\end{aligned}
$$

At our level of approximations, the position of the black hole horizon and consequently the black hole thermodynamics are not modified with respect to the RN magnetised solution. On the other hand, the geometry and the gauge field receive non-trivial corrections in the metric coefficient $w(r)$ and in the gauge component $A_{t}(r, \theta)$, which acquire new "mixed" contributions proportional to $g_{F} g_{R}$ due to the simultaneous presence of gauge and gravitational Chern-Simons terms. As far as we are aware, this is the first time that such mixed contributions have been found in this context, showing there is an interplay between gauge and gravitational Chern-Simons terms for determining the black hole geometry with axion hair. Thanks to this contribution, our solution generalises to a rotating, charged setting similar to configurations discussed in the works [1.5.5], [2.90]. 


\subsection{Phenomenological considerations}

\subsubsection{Black hole axion hair and light polarisation}

In the previous sections, we have seen that charged black holes can develop longrange axion hair, thanks to the gauge and gravitational Chern-Simons couplings contained in action (4..2). The Abelian charges carried by the black holes are not necessarily electromagnetic, and they can be associated with some additional gauge group motivated by string theory constructions, or dark forces associated with dark matter interactions. Even if the black hole configurations are charged under extra Abelian gauge groups that do not correspond to the electromagnetic $U(1)$ symmetry, we make the hypothesis that axions additionally couple with photons through a coupling term of the form:

$$
g_{\mathrm{EM}} \phi \mathcal{F}_{\mu \nu} \tilde{\mathcal{F}}^{\mu \nu}
$$

with $\mathcal{F}_{\mu \nu}$ being the electromagnetic $U(1)$ field strength and $g_{\mathrm{EM}}$ the coupling constant controlling the axion-photon interaction. The previous formula denotes a dimension five operator, that we weight with appropriate powers of the Planck mass (as in the previous sections) and regard $g_{\mathrm{EM}}$ as a dimensionless coupling. In case the black holes are electromagnetically charged, then $g_{\mathrm{EM}}=g_{F}$ with $g_{F}$ being the gauge coupling we discussed in the previous sections.

A possible way to detect a pseudoscalar black hole hair is to measure the bending of polarised light travelling in the black hole's surroundings. In fact, the parity violating coupling (4.72) differently deviates left-handed and right-handed photons, with a magnitude depending also on the values of $\left(g_{E M}, g_{F}, g_{R}\right)$ : thus, quantifying this effect could help in probing the magnitude of the Chern-Simons couplings characterising the theory. This effect has been studied in detail in the recent work [26] ] for the case of photons travelling through the cloud of light axions depositing on a rotating black hole ergosphere. Instead, here we study such bending effect in the case of light rays travelling through the long-range axion profile studied in the previous sections. We use the same approach and methods of [261], hence we refer the reader to [26]] for more details on the derivations. We study two cases, 
spherically symmetric and slowly rotating black holes, which can probe different sets of parameters.

\section{VLBI (Very Long Baseline Interferometry)}

Before presenting our results, we now briefly discuss how it is possible to detect polarised light from astrophysical sources. As it happens for radiation coming from AGNs, light coming from astrophysical radio sources is usually linearly polarised, since it is due to synchrotron emission; so, the possible detection of circularly polarised light could be the smoke gun for some exotic phenomena, like the ones discussed in this chapter. To detect circularly polarised waves we therefore need a dual-polarisation receiver with sufficiently high angular resolution.

According to the Rayleigh criterion, the angular separation through a circular aperture in the Fraunhofer limit ${ }^{\mathbb{W}}$ is given by [3:5]

$$
\delta \theta=1.22 \frac{\lambda}{D} \operatorname{rad}
$$

where $\lambda$ is the wavelength of the detected ray and $D$ is the diameter of the aperture/telescope. Recalling that

$$
1 \operatorname{rad}=2 \cdot 10^{6} \operatorname{arcsec},
$$

the angular resolution expressed in arcsec becomes

$$
\delta \theta=2.5 \cdot 10^{5} \frac{\lambda}{D} \text { arcsec. }
$$

To have an idea, if we take the reference value ${ }^{\square}$

$$
E_{\gamma}=1 \mathrm{GHz} \rightarrow \lambda=0.3 m
$$

\footnotetext{
${ }^{1}$ far from the diffraction object and on the focal plane of the lens.

${ }^{2}$ from now on, we will always consider a reference wavelength $\lambda=0.3 \mathrm{~m}$.
} 
and we consider an average telescope of diameter

$$
D=20 m
$$

we get a resulting angular resolution of

$$
\delta \theta=3.7 \cdot 10^{3} \operatorname{arcsec}
$$

which is clearly not enough accurate for detection on galactic distances.

For astrophysical measurements, it is therefore needed to drastically increase the angular resolution.

With the interferometry technique, multiple telescopes are disposed on an array and they are used at the same time, in order to greatly increase the angular resolution. In fact, the array acts as an effective single telescope, with diameter as large as the distance between the two farthest telescope in the array. For astronomy radio interferometry, the Very Long Baseline Interferometry (VLBI) consists in an array of telescopes on Earth with a resulting effective diameter of $D \approx$ $5 \cdot 10^{3} \mathrm{~km}$. Hence, the resulting, effective angular resolution is

$$
\delta \theta \approx 10^{-3} \text { arcsec. }
$$

For radioastronomy, an ulterior enhancement of the interferometry technique is the space VLBI (SVLBI), in which a ground based VLBI array is supported by orbiting telescopes and antennas, combined to create an effective telescope with a diameter bigger than the Earth's diameter. This is the case of Spektr-R ([326], [327]), a $10 \mathrm{~m}$ radio telescope orbiting around the Earth with perigee of $57.000 \mathrm{~km}$ and apogee of $320.000 \mathrm{~km}$, and which together with some other ground-based radio telescopes acts as an effective telescope up to $3 \cdot 10^{5} \mathrm{~km}$ of diameter, with a corresponding angular resolution of

$$
\delta \theta \approx 2 \cdot 10^{-4} \text { arcsec. }
$$

With such resolution, angular deflection caused by photons-axion interactions would be measurable, and that's why we believe this is a promising way for testing possible effects of cosmological ultralight scalar fields. 


\section{- Spherically symmetric black hole configurations}

We study the bending of light passing in proximity of spherically symmetric, dyonic black hole configurations with long-range axion hair, discussed in section 4.3. At large distances from the black hole's horizon, the black hole's geometry can be described by the Schwarzschild metric (since the corrections associated with the axion hair backreaction appears only at order $1 / r^{3}$ in a large $r$ expansion), with a long-range axion hair profile, that scales with radius as in equation (4.2. ):

$$
\Phi_{\text {dyon }}(r)=\frac{g_{F} P Q}{r_{+}} \frac{1}{r}+\mathcal{O}\left(\frac{1}{r^{2}}\right)
$$

with $r_{+}$being the position of the external horizon of a dyonic ReissnerNordström black hole (see equation (4.201)). We assume that light travels sufficiently far from the black hole, so that the Schwarzschild metric is a good approximation for the geometry. For simplicity, we also assume that the trajectory of photons lies in the equatorial plane of the black hole. Therefore, the system we are studying is described by the action:

$$
S=\int d^{4} x \sqrt{g}\left[\frac{1}{4} F_{\mu \nu} F^{\mu \nu}-\frac{g_{E M}}{8} \phi_{H} \tilde{F}_{\mu \nu} F^{\mu \nu}\right]
$$

where $F_{\mu \nu} F^{\mu \nu}$ is the photons propagation term, $F_{\mu \nu}$ is the standard electromagnetic field strength $\left(F_{\mu \nu}=\nabla_{[\mu} A_{\nu]}\right), \phi_{H}$ is the scalar hair solution around the black hole (in this case $\phi_{H}=\Phi_{\text {dyon }}$ as in equation (4.79)) and $g_{E M}$ is the coupling constant between the photon and the axion.

The $A_{\mu}$ equation of motion obtained from (4.801) gives us the following dispersion relation [26], 328]:

$$
k^{4}+g_{E M}^{2} \partial_{\mu} \phi \partial^{\mu} \phi k^{\nu} k_{\nu}=g_{E M}^{2}\left[k_{\mu} \partial^{\mu} \phi\right]^{2},
$$

with $k^{\mu}=\left(E_{\gamma}, k^{\alpha}\right)$ being the 4-momentum of the propagating photon. 
At first order in the coupling parameter, the dispersion relation becomes

$$
\frac{r\left[E_{\gamma}^{2}-k_{r}^{2}+r\left(k_{\theta}^{2}+k_{\varphi}^{2}\right)(2 M-r)\right]}{(2 M-r)}= \pm g_{E M}\left(k_{r} \frac{\partial \phi(r, \theta)}{\partial r}+k_{\theta} \frac{\partial \phi(r, \theta)}{\partial \theta}\right) .
$$

If we introduce the conserved angular momentum $L$

$$
k^{\varphi}=\frac{d \varphi}{d \lambda}=\frac{L}{r^{2}}
$$

and we notice that on the equatorial plane we also have

$$
k^{r}=\frac{d r}{d \lambda}=\dot{r}, \quad k^{\theta}=\frac{d \theta}{d \lambda}=0,
$$

we get

$$
\frac{r^{3}\left(E_{\gamma}^{2}-\dot{r}^{2}\right)+4 L^{2}(2 M-r)}{r^{2}}= \pm g_{E M}(2 M-r) \dot{r} \frac{\partial \phi(r, \theta)}{\partial r} .
$$

Solving for $\dot{r}$ we obtain:

$$
\begin{aligned}
\frac{d r}{d \lambda}= & \pm \frac{g_{E M}(2 M-r)}{2 r} \frac{\partial \phi(r, \theta)}{\partial r} \\
& \pm \sqrt{E_{\gamma}^{2}+\frac{L^{2}(2 M-r)}{r^{3}}+\frac{g_{E M}^{2}(2 M-r)^{2}}{4 r^{2}}\left(\frac{\partial \phi(r, \theta)}{\partial r}\right)^{2}}
\end{aligned}
$$

and taking the "+" root we get the orbit equation

$$
\begin{aligned}
\frac{d \varphi_{ \pm}}{d r}=\frac{1}{r^{2}}[ & \frac{ \pm g_{\mathrm{EM}}}{2 L}\left(1-\frac{2 M}{r}\right) \Phi_{\mathrm{dyon}}^{\prime}(r) \\
& +\sqrt{\left.\frac{E_{\gamma}^{2}}{L^{2}}+\frac{2 M-r}{r^{3}}+\frac{g_{\mathrm{EM}}^{2}}{4 L^{2}} \frac{(2 M-r)^{2}}{r^{2}} \Phi_{\mathrm{dyon}}^{\prime 2}(r)\right]^{-1}},
\end{aligned}
$$

where \pm refer to the two photon polarisations, while $\Phi_{\text {dyon }}$ is the axion profile given in equation (4.79). The contributions proportional to $g_{\mathrm{EM}}$ are associated with the axion-photon interactions: remarkably, in the presence of a long-range axion profile they can "distinguish" among the two different photon polarisations. 
The distance of closest approach $r_{0}$ of the light orbit to the black hole is given by the ratio of the energy and the angular momentum:

$$
\frac{E_{\gamma}}{L}=\frac{1}{r_{0}} \sqrt{\frac{r_{0}-2 M}{r_{0}}}
$$

and substituting it back into the orbit equation (4.87) we obtain

$$
\begin{aligned}
\frac{d \varphi}{d r}=\frac{1}{r^{2}}\{ & \pm \frac{g_{E M}}{2 E_{\gamma}}\left(1-\frac{2 M}{r}\right) \sqrt{\frac{1-\frac{2 M}{r_{0}}}{r_{0}^{2}}} \Phi_{\text {dyon }}^{\prime}(r) \\
& \left.+\sqrt{-\frac{1-\frac{2 M}{r}}{r^{2}}+\frac{1-\frac{2 M}{r_{0}}}{r_{0}^{2}}\left[1+\frac{g_{E M}^{2}}{4 E_{\gamma}^{2}} \frac{(r-2 M)^{2}}{r^{2}} \Phi_{\text {dyon }}^{\prime 2}(r)\right]}\right\}^{-1} .
\end{aligned}
$$

At this point, we use the explicit axion solution (4.79) and, for simplicity, we make the following variable redefinitions:

$$
x=\frac{r}{M}, \quad x_{0}=\frac{r_{0}}{M},
$$

to obtain

$$
\frac{d \varphi}{d x}=\frac{2 E_{\gamma} M^{2} x x_{0}^{3 / 2}}{\mp \gamma_{\Phi}+\sqrt{4 E_{\gamma}^{2} M^{4} x^{3}\left[x^{3}\left(x_{0}-2\right)-x x_{0}^{3}+2 x_{0}^{3}\right]+\gamma_{\Phi}^{2}}},
$$

with

$$
\gamma_{\Phi}=g_{F} g_{E M} \frac{P Q}{r_{+}}(x-2) \sqrt{x_{0}-2}
$$

To obtain the deflection angle, it is necessary to integrate the differential orbit equation (4.90) between the spatial infinity and the distance of closest approach, namely

$$
x_{i}=\infty, \quad x_{\text {end }}=x_{0} .
$$

However, we are especially interested in the difference $\Delta \varphi_{ \pm}$of angular deflection between right and left polarised photons: thus, we evaluate the quantity $\Delta \varphi_{ \pm}:$

$$
\left|\partial \varphi_{+}-\partial \varphi_{-}\right|=\frac{\gamma_{\Phi} x_{0}^{3 / 2}}{E_{\gamma} M^{2} x^{2}\left[x^{3}\left(x_{0}-2\right)-x x_{0}^{3}+2 x_{0}^{3}\right]},
$$


and assuming $x \gg x_{0}$ (i.e. the radial distance is much bigger than the closest approach distance) we are simply left with

$$
\left|\partial \varphi_{+}-\partial \varphi_{-}\right|=\frac{\gamma_{\Phi} x_{0}^{3 / 2}}{E_{\gamma} M^{2} x^{5}\left(x_{0}-2\right)} .
$$

Finally, integrating the above expression from $x_{i}$ to $x_{e n d}$ and replacing $\gamma_{\Phi}$ by its definition we arrive to the following simple formula

$$
\left|\Delta \varphi_{ \pm}^{\text {charged }}\right|=\frac{\left(2 x_{0}-3\right)}{x_{0}^{5 / 2} \sqrt{x_{0}-2}} \frac{g_{F} g_{\mathrm{EM}}}{6 E_{\gamma} M^{2}} \frac{P Q}{r_{+}} .
$$

Thus, a measurement of such quantity could probe and constrain the electromagnetic coupling of the axion to photons.

\section{- Slowly rotating black hole configurations}

We now study polarised light deflection around the slowly rotating configurations examined in section 4.4. Since we are especially interested in the effects of the gravitational Chern-Simons coupling, for the following calculations we consider neutral, slowly rotating black holes $(P=Q=0)$. At large distances from the black hole's horizon, the geometry (at leading order in a $1 / r$ expansion) is still well described by the Schwarzschild metric, and the axion field corresponds to a dipolar configuration given by

$$
\Phi_{\mathrm{rot}}(r, \theta)=\frac{5}{8} \frac{a}{M} \frac{g_{R}}{r^{2}} \cos \theta+\mathcal{O}\left(\frac{1}{r^{3}}\right) .
$$

As we did in the previous case, we now study the trajectories of photons close to the black hole; however, since in this case the axion field has a dipole structure, we do not consider trajectories lying on the equatorial plane, but rather on the more convenient plane $(\theta=\pi / 6)$. Proceeding as we explained in the spherically symmetric case, we find that the angular deflection of 
polarised photons is governed by the following equation

$$
\begin{aligned}
\frac{d \varphi_{ \pm}}{d r}=\frac{2}{r_{0}^{2}}[ & \pm \alpha\left(1-\frac{2 M}{r}\right) \sqrt{\frac{1-\frac{2 M}{r_{0}}}{r_{0}^{2}}} \\
& \left.+\sqrt{-\frac{1-\frac{2 M}{r}}{r^{2}}+\frac{1-\frac{2 M}{r_{0}}}{r_{0}^{2}}\left(1+\frac{\alpha^{2}(r-2 M)^{2}}{r^{2}}\right)}\right]^{-1}
\end{aligned}
$$

where $r_{0}$ is the closest approach distance of the photon to the black hole see equation (4.88) - and the parameter $\alpha$ is defined as

$$
\alpha=\frac{g_{\mathrm{EM}}}{2 E_{\gamma}} \frac{\partial \Phi_{\mathrm{rot}}(r, \pi / 6)}{\partial r} .
$$

From the previous expressions, we see that different photon polarisations are bent to different angles by interactions with the axion field. Integrating over the radial distance, the difference $\Delta \varphi_{ \pm}$of the angular deflection for left-handed and right-handed photons now reads

$$
\left|\Delta \varphi_{ \pm}^{\mathrm{rot}}\right|=\frac{\sqrt{3}}{16} \frac{\left(5 x_{0}-8\right)}{x_{0}^{7 / 2} \sqrt{x_{0}-2}} \frac{a g_{R} g_{\mathrm{EM}}}{E_{\gamma} M^{4}} .
$$

Interestingly, comparing with its analogous (4.96) in the previous case, equation $(4.100)$ can probe a different set of parameters, potentially providing constrains also on the axion-gravity Chern-Simons coupling $g_{R}$.

To summarise, we find compact formulas, equations (4.96) and (4.100), for the difference of angular deviation of right-handed and left-handed photons travelling through a region containing black hole's axion hair, as described in sections 4.3 and 4.4. These formulas can probe different parameters: while in the spherically symmetric case the polarised light bending can probe couplings between axions and gauge Chern-Simons terms, in the slowly rotating case we can also probe gravitational Chern-Simons interactions - a coupling that as far as we are aware is not probed by direct axion experiments. As far as we are aware, future astronomical observations based on radio astronomy can achieve an angular resolution of order $\Delta \phi \sim 10^{-4}[\overline{26 \mathrm{G}}]$, and it therefore would be interesting to quantitatively estimate 
at what extent these precise measurements can test the existence and properties of black hole axionic hair, and their couplings with photons and/or gravity as suggested in the previous discussion.

\subsubsection{ISCOs}

As a second application of our findings, we now compute the ISCOs (Innermost Stable Circular Orbits) of the dyonic black hole configuration with axion hair we derived and discussed in section 4.33, including the effects of non-minimal couplings of axions with gravity (see Lagrangian ( $(\pi, \mathbb{T})$ ). The properties of ISCOs can be interesting for a phenomenological characterization of the geometrical features of the system under investigation. Since they depend on the properties of the configuration relatively near the black hole's horizon, they can be sensitive to the effects of Chern-Simons couplings, or of the derivative non-minimal couplings with gravity. For an asymptotically flat, spherically symmetric background we have the general line element

$$
d s^{2}=-e^{f(r)} d t^{2}+e^{s(r)} d r^{2}+r^{2} d \theta^{2}+r^{2} \sin ^{2} \theta d \varphi^{2}
$$

Thanks to spherical symmetry, we can choose to calculate the ISCOs trajectories on the convenient equatorial plane $(\theta=\pi / 2)$.

We introduce the 4-velocity vector

$$
u^{\mu}=\left\{u^{t}, u^{t}, u^{\theta}, u^{\varphi}\right\}
$$

and we can therefore define the conserved energy $E$ and the angular momentum $L$ as

$$
E=e^{f(r)} u^{t}, \quad L=r^{2} u^{\varphi}
$$

From the normalisation condition

$$
u^{\mu} u_{\mu}=-1
$$


using the relations (4.103) and the fact that on the ISCOs the radial velocity vanishes, we get the following equation

$$
e^{-[f(r)+s(r)]} E^{2}-\frac{e^{-s(r)}\left(L^{2}+R^{2}\right)}{r^{2}}=0
$$

which can be simplified and rewritten as

$$
E^{2}=e^{f(r)} \frac{L^{2}+r^{2}}{r^{2}}
$$

At this point, we define the effective potential $V(r)$ as

$$
V(r)=e^{f(r)} \frac{L^{2}+r^{2}}{r^{2}}
$$

and its first and second derivatives read:

$$
\begin{aligned}
& \frac{d V}{d r}=\left[r\left(L^{2}+r^{2}\right) f^{\prime}(r)-2 L^{2}\right] \frac{e^{f(r)}}{r^{3}} \\
& \frac{d^{2} V}{d r^{2}}=\left\{r\left[r\left(L^{2}+r^{2}\right) f^{\prime \prime}(r)+r\left(L^{2}+r^{2}\right) f^{\prime}(r)^{2}-4 L^{2} f^{\prime}(r)\right]+6 L^{2}\right\} \frac{e^{f(r)}}{r^{4}} .
\end{aligned}
$$

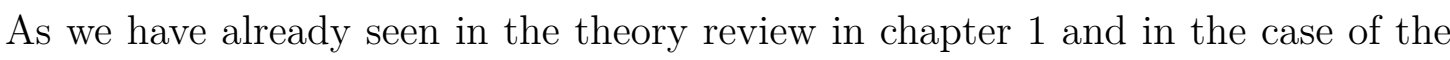
disformal black hole solution in chapter 2 , on the ISCO trajectory both the first and the second derivative of the effective potential vanish. From the first condition, we get

$$
r\left(L^{2}+r^{2}\right) f^{\prime}(r)-2 L^{2}=0
$$

which leads us to the conserved angular momentum on the ISCO:

$$
L_{I}=\left.\frac{r^{\frac{3}{2}} \sqrt{f^{\prime}(r)}}{\sqrt{2-r f^{\prime}(r)}}\right|_{r_{I S C O}} .
$$

Inserting the last expression back into the equation (4.06)), we get the Energy of the ISCO:

$$
E_{I}=\left.e^{\frac{f(r)}{2}} \sqrt{\frac{2}{2-r f^{\prime}(r)}}\right|_{I_{I S C O}} .
$$


Setting the second derivative of the potential to zero and using the above results we get

$$
3 f^{\prime}(r)-r f^{\prime}(r)^{2}+r f^{\prime \prime}(r)^{2}=0
$$

which can be solved with respect to the variable $r$ to obtain the ISCO's radius.

Finally, an expression for the angular frequency

$$
\omega=\frac{u^{\varphi}}{u^{t}}
$$

of the ISCO is given by

$$
\omega_{I}=\frac{L_{I}}{r^{2}} \frac{e^{A(r)}}{E_{I}}=\left.e^{\frac{A(r)}{2}} \sqrt{\frac{A^{\prime}(r)}{2 r}}\right|_{r_{I S C O}} .
$$

Comparing (4.10س) with (4.3.3), we have

$$
f(r)=\log [F(r)]
$$

and hence we can solve (4.12) to get the ISCO radius; for an analytical solution, we need to perform an expansion for small magnetic and electric charge $P$ and $Q$, and therefore we define the following expansion parameter:

$$
q^{2}=P^{2}+Q^{2}
$$

So, the quantity $P^{2} Q^{2}$ is an $\mathcal{O}\left(q^{4}\right)$ term, and looking at (4.3.3) we notice that we have to push our perturbative expansion up to order $\mathcal{O}\left(\epsilon^{6}\right)$ to glimpse the effects of the Chern-Simons coupling $g_{F}$ and the ones from the non-minimal couplings with gravity $\lambda$. Moreover, to solve the ISCO equation we use the following ansatz:

$$
r_{I S C O}=r_{S}+q^{2} r_{2}+q^{4} r_{4}+q^{6} r_{6}+\mathcal{O}\left(q^{8}\right)
$$

where $r_{S}$ is the radius of the Schwarzschild ISCO

$$
r_{S}=6 M
$$


and $r_{2,4,6}$ are the corrections at order $\mathcal{O}\left(q^{2}\right), \mathcal{O}\left(q^{4}\right)$ and $\mathcal{O}\left(q^{6}\right)$, respectively. With these approximations, the ISCO radius results

$$
\begin{aligned}
r_{I S C O}= & 6 M-\frac{P^{2}+Q^{2}}{2 M}-\frac{19\left(P^{2}+Q^{2}\right)^{2}}{72 M^{3}}-\frac{5\left(P^{2}+Q^{2}\right)^{3}}{48 M^{5}} \\
& +g_{F}^{2} P^{2} Q^{2}\left[\frac{19}{144}+\left(P^{2}+Q^{2}\right) \frac{\left(49410 M^{2}-193 \lambda\right)}{466560 M^{7}}\right] .
\end{aligned}
$$

The first line contains the General Relativity expression for the ISCO radius: the Schwarzschild result $(6 M)$ and the first corrections in small values of the charges associated with a dyonic Reissner-Nordström configuration. Instead, the second line contains the contributions associated with the presence of black hole's axion hair, the Chern-Simons terms, and non-minimal coupling with gravity. For small values of the charges, these contributions appear only at order $\mathcal{O}\left(q^{4}\right)$, thus it can be difficult to use the properties of ISCO to reveal the existence of axion hair. To conclude, we report the angular frequency associated with the ISCO trajectory

$$
\begin{aligned}
\omega_{I S C O}= & +\frac{1}{6 \sqrt{6} M}+\frac{7\left(P^{2}+Q^{2}\right)^{2}}{144 \sqrt{6} M^{3}}+\frac{49\left(P^{2}+Q^{2}\right)^{4}}{2304 \sqrt{6} M^{5}}+\frac{5489\left(P^{2}+Q^{2}\right)^{6}}{497664 \sqrt{6} M^{7}} \\
& +g_{F}^{2} P^{2} Q^{2}\left[-\frac{1}{216 \sqrt{6} M^{5}}+\left(P^{2}+Q^{2}\right)^{2}\left(\frac{11 \lambda}{699840 \sqrt{6} M^{9}}-\frac{47}{7776 \sqrt{6} M^{7}}\right)\right] .
\end{aligned}
$$

Again, the first term corresponds to the Schwarzschild result, while the contributions from the scalar field appear at order $\mathcal{O}\left(q^{4}\right)$.

\subsubsection{Scalar hair in Active Galactic Nuclei}

To conclude this chapter, we now give a very short insight on some possible connections and implications with astrophysical systems of the results we have derived and discussed in the previous sections.

Astrophysical black holes are not isolated, static objects which live in vacuum, but rather they are dynamical systems which interact with the surrounding matter and radiation, as we know that supermassive black holes sit at the center of most (or even all) galaxies. Active Galactic Nuclei (AGNs) are well known and observed highly dynamical systems where electromagnetic radiation is presumably emitted due to the accretion of matter by a supermassive black hole in the center 
of the host galaxy [32.9, 330]. The matter accretion is caused by the gravitational attraction of the black hole on the surrounding area; in a AGN, the black hole in the center of the host galaxy acquires surrounding matter "either by pulling interstellar plasma into its vicinity, or by disrupting passing stars and smearing their matter out around itself" [130].

Since in both cases the gas has a huge angular momentum, the black hole does not radially and directly "swallow" it, rather the gas starts fluctuating and orbiting forming a disk around the hole. At large distances $(r>100 M)$ the inclination of the disk depends on the direction of the original angular momentum of the gas; however, at closer distances $(r<100 M)$ the hole exerts such a strong attraction that the disk is forced to lay on the equatorial plane [13.9]. Near the hole, gravitational energy is continually converted into thermal energy, making the surrounding plasma highly ionized. For this reason, it can be well approximated and modeled as a perfect conductor, where electric charges can freely travel and the magnetic lines are frozen into it. The geometry of the magnetic field lines around the black hole is generically called magnetosphere: a detailed discussion on the magnetosphere dynamics is beyond the scopes of this work, so for a more exhaustive discussion on the magnetic field lines dynamics see [1;0]].

Remarkably, the presence and origin of strong magnetic fields around black holes and other compact objects are not fully understood yet. In the last decades, many explanations have been proposed; however, it is commonly accepted that despite of the unclear origin and nature of such magnetic fields, they are involved in the emission of high energy jets from compact objects.

On the basis of the model proposed by Goldreich and Julian [3:3] in 1969 for the axisymetric magnetosphere surrounding a pulsar, in 1977 Blandford and Znajek [217] argued that a rotating black hole surrounded by ionized plasma and threaded by a sufficiently strong magnetic field could efficiently extract electromagnetic energy from the magnetic field lines, through the emission of jets. The model proposed by Blandford and Znajek is based on a relativistic force-free magnetosphere configuration, where the plasma pressure is assumed to be small compared to the magnetic pressure (see [332-B34] for more details about the force-free condition). On the basis of this argument, in the last 20 years a lot of work has been done in the understanding and modeling of the force-free magnetosphere dynamics, with 
both analytical results and numerical simulations [1.34, 335-338].

However, although a definitive and completely exhaustive model does not exist yet, the relativistic force-free magnetohydrodynamics has been largely accepted to explain the energy extraction from a Kerr black hole and powering AGNs, relativistic jets in X-ray binaries and gamma-ray bursts [334].

Without motivating the origin and the nature of the magnetic field, we now consider a rotating Kerr black hole immersed in an external magnetic field, whose lines are frozen into the plasma surrounding the black hole. Using a $3+1$ spacetime decomposition [1.30, 1.3.9], for a Zero Angular Momentum Observer (ZAMO) we choose a spatial local coordinate basis $\mathcal{B}=\left\{\vec{e}_{r}, \vec{e}_{\theta}, \vec{e}_{\varphi}\right\}$. By definition, the chosen observer is at rest in the rotating space around the black hole. However, since the magnetic field lines do not rotate at the same angular velocity of the observer, they will feel an effective electric field induced by the rotating magnetic field. On the chosen basis, we can decompose the electric and the magnetic fields into a toroidal (denoted with T) and poloidal (denoted with P) components:

$$
\begin{array}{lll}
\vec{E}=\vec{E}^{T}+\vec{E}^{P}, & \vec{E}^{T}=E^{\varphi} \vec{e}_{\varphi}, & \vec{E}^{P}=E^{r} \vec{e}_{r}+E^{\theta} \vec{e}_{\theta} \\
\vec{B}=\vec{B}^{T}+\vec{B}^{P}, & \vec{B}^{T}=B^{\varphi} \vec{e}_{\varphi}, & \vec{B}^{P}=B^{r} \vec{e}_{r}+B^{\theta} \vec{e}_{\theta} .
\end{array}
$$

Since the vector $\vec{e}_{\varphi}$ is orthogonal to the field lines angular velocity, we have

$$
\vec{E}^{T}=0
$$

and hence the electric field is purely poloidal and it is given by

$$
\vec{E}^{P}=-\vec{v}_{F} \times \vec{B}^{P}
$$

Denoting with $\omega$ and $\Omega$ the angular velocity of the ZAMO and the field lines respectively, we have

$$
\vec{v}_{F}=\frac{1}{\alpha}(\Omega-\omega) \tilde{\omega} \vec{e}_{\varphi}
$$

with

$$
\alpha=\sqrt{\frac{\rho \Delta}{\Sigma}}, \quad \tilde{\omega}=\sqrt{\frac{\Sigma}{\rho}} \sin \theta, \quad \Sigma=\left(r^{2}+a^{2}\right)^{2}-a^{2} \Delta \sin ^{2} \theta .
$$

So, from the expression of the poloidal electric field (4.52:3) we get 


$$
\vec{E} \cdot \vec{B}=0
$$

which means that there is no Lorentz force carrying charges along the magnetic field lines (thus the name force-free). Macroscopically, the surrounding plasma is acting as a perfect conductor around the hole, rearranging the interior charge distribution to completely screen the electric field:

$$
\vec{E}_{t o t}=\vec{E}+\vec{v} \times \vec{B}=0
$$

which is the same result of (4.T2.3).

Thus, inside the plasma, the electromagnetic Chern-Simons term

$$
F_{\mu \nu} \tilde{F}^{\mu \nu}=-\vec{E} \cdot \vec{B}
$$

vanishes, since the electric field does. Consequently, in order to preserve and motivate a scalar hair arising from a Chern-Simons term like (4.28), we have three different possible scenarios.

In the simplest case, the Chern-Simons term which sources the axion hair $(4.28)$ and $(4.67)$ is not the electromagnetic one, namely $F_{\mu \nu} \neq F_{\mu \nu}^{E M}$, and hence the force-free magnetosphere screening does not affect the scalar hair.

A second possibility consists in having the force-free plasma screening the electric field and the Chern-Simons term only in the black hole's nearby area. The screening is effective as long as the force-free condition is satisfied, i.e. so long as the plasma is sufficiently highly ionized to be considered a perfect conductor and its pressure is negligible when compared to the magnetic one. Thus, it is reasonable to expect that these conditions are satisfied in the neighborhood of the black hole, where the gravitational attraction is very intense and the magnetic fields are extremely strong. On the other hand, far from the black hole the force-free condition could no longer be satisfied; in that case, the electric field may be unscreened, hence having a non vanishing Chern-Simons coupling term and consequently a scalar hair surrounding the black hole (a pictorial and schematic representation of this scenario is given in figure 4.1). 


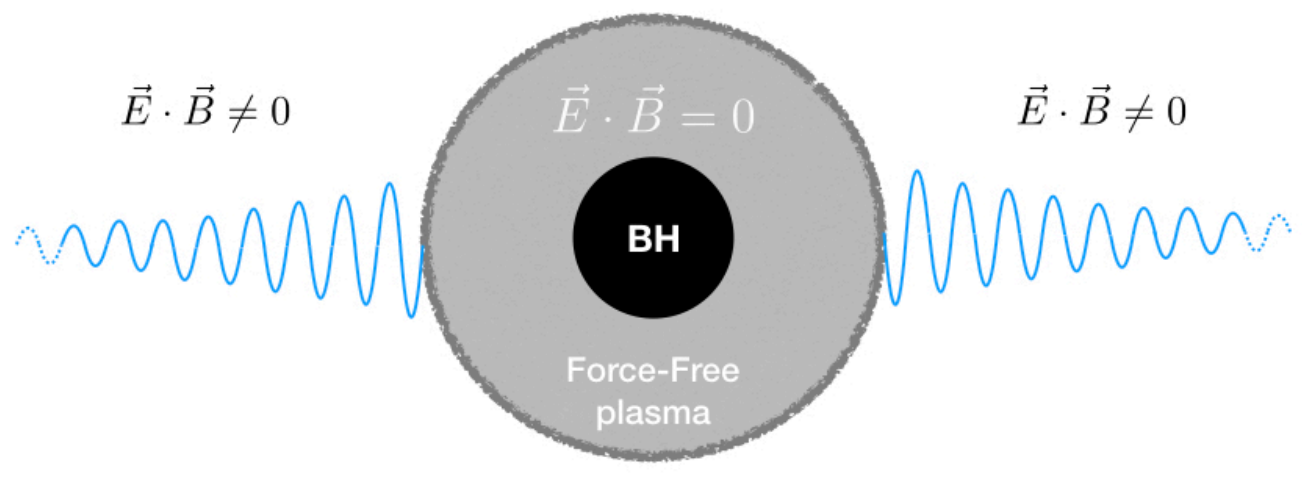

Figure 4.1: Force-Free plasma (grey area) surrounding the black hole (black area): inside the force-free area, the electric field is screened by the plasma, which acts as a perfect conductor. Thus, inside the force-free area there is no source for the scalar field (blue line), and no hair is developed. Instead, outside the grey area there is an unscreened, non vanishing electric field which can act as a source for the scalar field through a Chern-Simons coupling term. So, the black hole develops a scalar hair outside the force-free area.

The third possible scenario consists in having the force-free plasma screening the electric field and consequentely the axion hair from a radial effective screening distance $r_{e}$ such that $r_{e}>r_{+}$. In this case, the black hole would develop a scalar hair in the region between the event horizon and the effective screening distance $r_{e}$ (a pictorial and schematic representation of this scenario is given in figure 4.2. It is interesting to notice that this configuration naturally provides the black hole bomb scenario conditions, since the force-free plasma acts as a perfect screen for the scalar field at distances $r_{e}>r_{+}$; thus, as future investigations it would be interesting to study a non static scalar field, in order to see whether and how superradiance and eventually superradiant instability are triggered. 


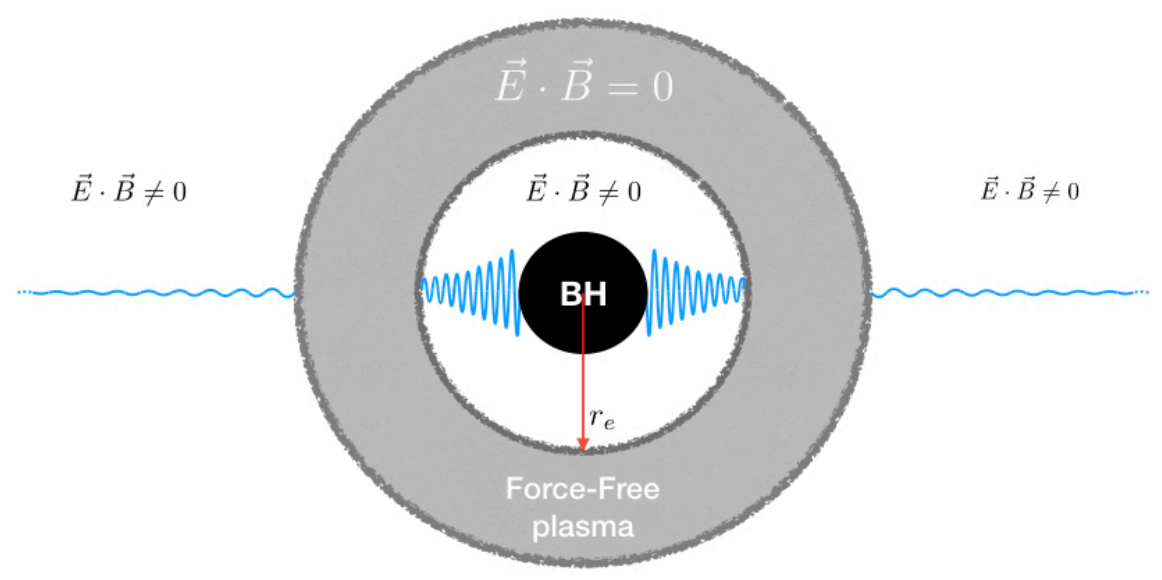

Figure 4.2: $\quad$ The force-free plasma (grey area) is effective from an effective screening distance $r_{e}$. In the region enclosed between the event horizon and the force-free area $\left(r_{H} \leq r \leq r_{e}\right)$ the electric field is non vanishing, hence sourcing a scalar hair (blue line) surrounding the black hole (black area). Inside the force-free region, the plasma totally screens the electric field, and consequently the Chern-Simons term proportional to $\vec{E} \cdot \vec{B}$ vanishes, screening the black hole's scalar hair.

\subsection{Conclusions}

In this chapter, we studied spherically symmetric and slowly rotating charged black hole configurations with long-range axion hair. We focused on Einstein-Maxwell theories equipped with an axion Lagrangian that preserves a shift symmetry for the axion field. Gauge and gravitational Chern-Simons couplings (equation (4.D)) are essential for evading no-hair theorems, and lead to long-range axion profiles. We extended known black hole solutions to cases where additional derivative couplings of axion to curvature appear (4.3), and to situations in which both gauge and gravitational Chern-Simons couplings are simultaneously present (4.67). In all cases, we determined analytical solutions at leading order in the coupling constants involved, determining how the axion profile backreacts on the metric and the gauge field. The metric remains regular outside the outer horizon, and the position of such horizon is increased with respect to dyonic solutions in Einstein-Maxwell 
gravity with the same asymptotic conserved charges. Moreover, the solution for the electric potential is modified with respect to the standard case due to the effect of the gauge Chern-Simons couplings. Furthermore, for the spherically symmetric configurations we analysed, we showed that the black hole thermodynamics Smarr formula holds even with contributions from the axion backreaction appearing at leading order in the coupling constants. Furthermore, to make contact with phenomenology, we studied two possible consequences of our findings. First, we studied how axion hair can induced a polarization dependent deviation of photons travelling within the range of the axion profile around a black hole. Then, we investigated the properties of ISCOs around the spherically symmetric configurations we studied. At the end, we gave a brief and intuitive suggestion (4.5.3) of the possible consequences and interactions of the black hole configurations we studied in the contest of astrophysical scenarios like AGNs.

For further investigations, it would be interesting in the future to study the stability of the considered systems under small perturbations of the fields involved, and investigate possible parity breaking effects - induced by the Chern-Simons couplings - in the dynamics of fluctuations around these geometries.

In conclusions, this chapter provides examples of black holes with surrounding long-range scalar hair, proposing possible detectable effects to test such axion fields. As already largely discussed, these axions appear in both particle physics and string theory, and they could have great impact on cosmology: thus, the importance of finding way to test their existence and nature, with black holes being the best candidates to spot them. 
4. BLACK HOLE HAIR 


\section{Chapter 5}

\section{Black hole perturbations}

Quasi Normal Modes (QNMs) are a detectable, distinctive feature of black holes which can provide a strong tool to identify and test gravity theories through gravitational waves detection. In particular, for binary systems merging into a single black hole the ringdown phase is characterized by the emission of Quasi Normal Modes. Thus, for any black hole solution to be tested with gravitational waves it is important to study its perturbation theory, looking for possible distinctive effects from General Relativity. In this chapter, we study the linear perturbation theory of an electromagnetic Reissner-Nordström black hole solution with an external scalar field, also including a Chern-Simons coupling between the scalar field and the gauge vector. Interestingly, differently to the Schwarzschild case, we find that axial (odd) and polar (even) perturbations are not decoupled, leading to a Regge-Wheeler perturbation equation involving terms with different behaviour under parity transformation.

\subsection{Introduction and motivations}

To conclude this work, we now briefly touch on a currently very popular and fascinating topic for both high energy physics and astrophysics: the black holes Quasi Normal Modes (QNMs). Originally studied to investigate black holes stability, QNMs have recently become a largely studied research area in high energy physics as well, due to the gauge-gravity dualities and the developments of condensed 
matter physics. With a simplistic definition, QNMs are the energy dissipation's modes of a perturbed object or field. When perturbed, objects/fields begin to vibrate with their proper (normal) vibration frequencies, which are hamiltonian eigenstates and therefore infinitely long-lived solutions; so, if the system was isolate and no dissipation occurred, the object would vibrate forever. However, in many physical systems dissipative effects are usually unavoidable, and the vibration modes are not infinitely long-lived but they rather decay in time, being therefore called quasi-normal. As it happens in everyday life with glasses and bells (whose normal frequencies are usually in the audible spectrum) and other objects, the same physical behaviour is manifested by black holes and quantum fields. As the glass QNMs depend on the glass structure only, in the same way the black holes QNMs depend only on the structure of the black hole they have been originated from. For this reason, black hole QNMs can be considered a recognition sign of the ringing black hole. And here it comes the great news: since black holes are usually (at least in General Relativity!) described by quite few parameters, hence the black holes QNMs are described by the same few parameters as well! According to the already largely discussed no-hair theorem, astrophysical black holes are usually described by 3 parameters: mass, angular momentum and charge ${ }^{m}$; consequently, black holes QNMs have to be fully described by these three quantities. However, as we have seen in the previous chapters, if we considered gravity theories different from GR where extra fields are taken into account in the action, the corresponding black hole solutions could carry additional degrees of freedom, which would therefore be carried by the QNMs as well.

So, it is now clear how black holes QNMs are a distinctive and authentic "footprint" of the black holes, and their direct detections would therefore give us information on the nature of the "ringing" black hole. But how are astrophysical black holes dissipative systems? Why are the vibration modes quasi-normal and not normal? An exhaustive answer to this question is beyond the aim of this chapter, but a quick and intuitively answer can be found if we think at the screening nature of the black hole event horizon, sometimes described as perfect dissipative

\footnotetext{
${ }^{1}$ In Einstein-Maxwell theory, the black hole charge is the electromagnetic one. However, we could generalise it to a generic charge associated with the corresponding symmetry of the action whose the black hole is solution.
} 
membrane (membrane paradigm [[130]) surrounding the causally inaccessible inner region. From this point of view, the event horizon can be regarded as a totally dissipative surface, which allows no energy/information to escape from it; in this sense, black holes are extremely dissipative systems, hence supporting quasinormal modes rather than normal.

In this chapter, we will show how the physical system described in the previous chapter has interesting features related with black hole perturbations, and consequently with the black hole's QNMs. So, before discussing our results, we want to give a very brief overview of this huge research area, focussing our attention on possible connections with detectable strong gravity phenomena and in particular with gravitational waves detections. In fact, as confirmed by GW150914 and the other GWs detections, immediately after the merger/collapse of a binary system and the formation of a single black hole, the system undergoes to a phase called ringdown, where the newly formed black hole settles down through the propagation of its QNMs. Thus, from the last moments of the detected GW signals from binary systems merging into a single black hole, we can extract the QNMs spectrum and consequently the physical parameters describing the new black hole. As shown in Figure 5. 5 , immediately after the merger of the two black holes there is a sharp decay of the strain's amplitude. After the merger, the ringdown phase begins: during this phase the black hole vibrates according to its QNMs, which are the damped, sinusoidal modes shown in the red area in Figure 5.2. Again, we stress the fact that since the QNMs depend only on the black hole's physical parameters, a specific data analysis of a ringdown signal such the one in Figure 5.1 can give us accurate informations about the newly formed black hole. For this reason, it is important to investigate whether in modified gravity theories black hole solutions exist and how their QNMs spectra look like. In this way, using the GWs data we could be able to test these theories against GR, looking for any hint of unexplored physics. 


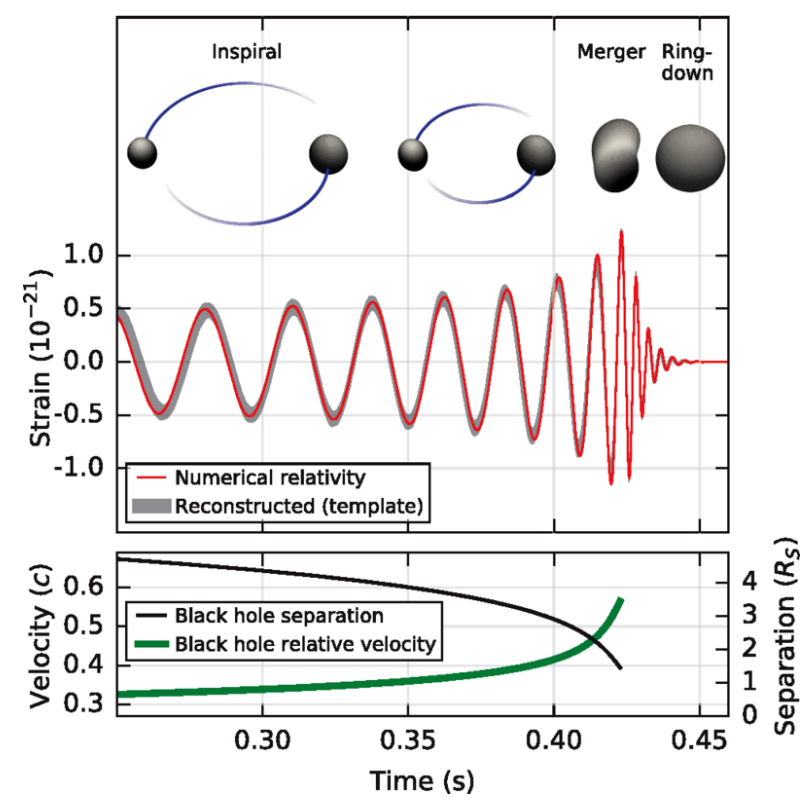

Figure 5.1: The gravitational wave signal of GW150914. Image from [3].

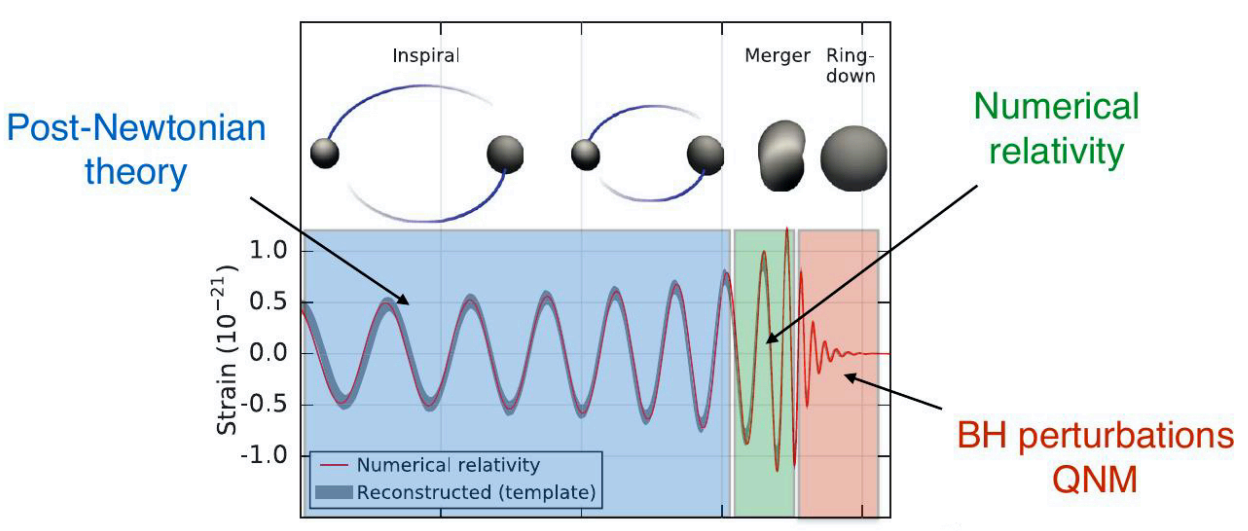

Figure 5.2: The picture shows how the theoretical Gravitational Wave signal is obtained for the different stages of the collapse of two rotating black holes into a single one: PN (Post-Newtonian) theory for the inspiral phase (blue region), numerical relativity for the merger phase (green region), black hole perturbation theory for the ringdown (red area). Image from the talk Gravitational Waves: a new window to explore the Universe by Philippe Jetzer at Joint annual meeting of the Swiss and Austrian Physical society in 201\%. 
Before going through the calculation and the main results of this chapter, we will provide a very brief historical review of this vast topic, quoting the milestones which have lead to the main developments in black holes QNMs research ${ }^{\mathbb{}}$ in the last sixty years. However, since this is not meant to be an exhaustive review, many important details and historically significant works will be omitted: for recent and exhaustive literature reviews on the topic, see [339, 340], while for older comprehensive theory review on black holes QNMs see [341-343].

Based on [33:9], we report the following milestones:

- 1957: Regge and Wheeler [18] firstly study the gravitational perturbations of Schwarzschild black holes. This is the beginning of the black hole perturbation theory.

- 1961: Newman and Penrose [344] develop a new formalism to treat general relativity in terms of spinor notation.

- 1963: Kerr finds an exact solution of Einstein's equations describing a rotating black hole [40].

- 1970: Zerilli extends the Regge-Wheeler analysis to generic perturbations of Schwarzschild black holes [345, 346].

- 1970: Vishveshwara [347] numerically studies the scattering of gravitational waves with a Schwarzschild black hole: at late times, the waveform is formed by damped sinusoids. The term ringdown is introduced to describe such late phase.

\footnotetext{
${ }^{1}$ In the following milestones list we have reported only achievements inherent with black holes QNMs. For an accurate review on other important QNMs research areas, see [3.3.9].
} 
- 1971: Press shows that ringdown waves are the free oscillation modes of the black hole [348].

- 1971: Davis et al firstly calculate (using black hole perturbation theory) the gravitational waves emission due to a test particle falling into a Schwarzschild black hole [349].

- 1972: Goebel describes black holes QNMs as gravitational waves [350]].

- 1972: Teukolski [251] shows the separability of perturbations equations on the Kerr geometry.

- 1974: Zerilli [35] studies perturbations of charged Reissner-Nordström black hole.

- 1975: Moncrief [352] develops a gauge invariant perturbation formalism to study electromagnetic perturbations.

- 1975: Chandrasekhar and Detweiler [35.3] numerically compute some QNMs frequencies.

- 1983: Chandrasekhar publishes a comprehensive monograph on black hole physics, containing an up-to-date review of black hole perturbation theory [341].

- 1983: Ferrari and Mashhoon analitically compute QNMs from black holes potentials [3.54]. 
- 1985: Stark and Piran [355] use numerical relativity to obtain the gravitational waves emission of a rotating system collapsing into a black hole.

- 1985: Leaver applies the continued fraction representations method to numerically compute black holes QNMs [356, 357].

- 1989: Echeverria [358] estimates the accuracy of extrapolating the black hole's mass and angular momentum from QNMs detections.

- 1993: Anninos et al. firstly simulate the collision of black holes with QNMs production during the ringdown phase [35.9].

- 1998: Flanagan and Hughes show the QNMs detection range in LIGO and LISA sensibilities [360].

- 1999: Kokkotas and Schmidt [342] and Nollert [34:3] publish detailed reviews on QNMs.

- 2003: Motl and Neitzke analytically compute highly damped QNMs using a monodromy technique [361].

- 2003: Kodama and Ishibashi generalise the Regge-Wheeler formalism to higher dimensional black holes [362].

- 2005: Pretorius fully simulates a stable evolution of a binary black hole system merging into a single black hole, with QNMs emitted in the ringdown phase [36:3]. 


\section{BLACK HOLE PERTURBATIONS}

- 2009-2015: black holes QNMs have been explored in many different modified gravity theories, such as massive gravity [364], Chern-Simons gravity [365] or with the addition of massive vector fields [366].

- 2015: GW150914 is the first gravitational waves detection event [3]. QNMs detection in the ringdown phase is in agreement with numerical and theory predictions.

As far as this work is concerned, the previous list was intentionally focused on QNMs of astrophysical black holes and their connections with gravitational waves astronomy. However, QNMs have also been largely investigated in other research areas. For example, starting from the 80's, many authors have been trying to clarify the connection between QNMs and quantum gravity; on such topic, we mention the work done by York [367], Hod [368], and Dreyer [36.9]. Moreover, in the last twenty years, QNMs have been living a new great season of interest, fueled by the development of the AdS/CFT correspondence. So, following the road opened by Maldacena [370], a lot of work has been done to apply QNMs results to strongly coupled quantum field theories [3r1-373], with a recent particular interest to condensed matter theories as well [374-B76].

The fact that a sixty years old topic is still current and fashionable is really remarkable. In this work, we are interested in QNMs for astrophysical black holes in modified gravity theories. Since we now have direct access to the single black hole gravitational waves emission (during the ringdown phase), it is possible to test and falsify gravity theories according to their QNMs spectra, comparing the predicted ones with the detections. With this in mind, we are interested in finding any peculiar feature about black hole gravitational perturbations which could potentially be tested with future observations and detections. In particular, in this chapter we will focus our attention on parity violating effects. As we will discuss, the Schwarzschild and Kerr backgrounds spontaneously decouple perturbations with different parity, leading to two independent master equations (one 
for the parity even perturbations and one for the parity odd ones). For this reason, it is interesting to investigate whether this "parity decoupling" happens also in other backgrounds, when extra fields and extra couplings are taken into account.

In this work we will not explicitly compute the black holes QNMs, but we rather investigate the master equations they are solution of. For a very brief review on how QNMs are computed, see [339, 342].

In general, QNMs are solutions of an eigenvalue problem with opportune boundary conditions. As we will see in the next sections, the study of black hole perturbations at linear order can be reduced to a Schrödinger-like equation

$$
\frac{d^{2} \Psi_{s}}{d r_{*}^{2}}+\left(\omega^{2}-V_{s}\right) \Psi_{s}=0
$$

with $s=0,1,2$ being the spin of the perturbed field and $V_{s}$ being the characteristic potential. For conveniency, the perturbation equation has been written using the radial tortoise coordinate $r_{*}$ and in the Fourier space, with $\omega=-i \partial_{t}$. Remarkably, equation (5.D) has the same structure for scalar, vector and tensor fields, making the study of the problem absolutely general. Equation (5.D) defines an eigenvalue problem for frequencies $\omega$ : QNMs are eigenfunctions which satisfy specific boundary conditions at spatial infinity (far zone) and on the black hole's horizon. In particular, QNMs are required to be decaying at infinity $\left(r_{*} \rightarrow \infty\right)$ and purely ingoing on the horizon $\left(r_{*} \rightarrow-\infty\right)$, in accordance with the perfectly dissipative behaviour of the horizon (membrane paradigm). As we will show, after expanding field's perturbations with an opportune spherical harmonics basis it is possible to separate them into two sectors (even and odd) according to their behaviour under parity transformations. In Schwarzschild background, the two parity sectors are independent/decoupled, and it is therefore possible to reduce the perturbations equations to a couple of independent Schrödinger-like master equations (with the form of equation (5.0)): the parity odd equation is usually named Regge-Wheeler equation, while the even one is usually referred to as the Zerilli equation. Interestingly, in the far zone (where $\omega r \gg 1$ ) the Regge-Wheeler and the Zerilli equations both decay as $1 / r$, and it can be shown (see [377]) that they are isospectra, hence admitting the same QNMs. So, it is important to 
investigate whether "exotic" gravity theories can be reduced to the same couple of master equations as in GR, and eventually compute their QNMs spectra to be compared with the gravitational waves signal from black holes ringdown.

We are now ready to move to the next sections, where we have explicitly computed the Regge-Wheeler master equation for various backgrounds, with particular interest for the Reissner-Nordström geometry. We are aware that a large part of the scientific community believes that charged black holes are not likely to be a large population, since in astrophysical settlements they presumably undergo to a neutralisation process. However, since the neutralisation time can be much longer than the average life of a binary neutron star system, we think it is not cautious to completely exclude them from investigations. Moreover, as clearly discussed in [378], the Reissner-Nordström and the Kerr-Newman black holes families are also the ones associated with a possible $U(1)$ hidden sector, which is still one the most promising candidates for dark matter and dark energy [4, 379, 380]. Therefore, we believe that the study of charged black holes should not be merely considered a speculative and unphysical investigation.

The remaining part of this chapter is structured as follows.

In section 52 we describe and discuss the global physical system we are considering, and we introduce the basis of black hole perturbation theory. In section 5.3 we choose the convenient gauge "RW" (Regge-Wheleer) and we decompose the perturbations in tensorial spherical harmonics, defining parity even perturbations and parity odd ones. In section 5.3 .0 we review the Schwarzschild case, studying the gravitational linear perturbation which lead to the independent Regge-Wheeler and Zerilli equations. In sections 5.4 and 5.5 we respectively study the electric and the magnetic Reissner-Nordström perturbations, deriving the Regge-Wheeler equations for both the gravitational and the electromagnetic perturbations. As we will see, the magnetic case reveals an interesting peculiar feature. Then, in sections 5.6 and 5.7 we study tensor, vector and scalar perturbations for electric and magnetic Reissner-Nordström backgrounds respectively, with an extra scalar-vector, Chern-Simons coupling included in the action. Finally, in section 5.8 we conclude with a brief discussion on possible implications and further developments. 


\subsection{Set-up}

In this chapter, we study the fields perturbations of a spherically symmetric black hole coupled to a vector field and a scalar (axion like) field. In particular, we consider black hole solutions of the Einstein-Maxwell action

$$
S_{E M}=\int d^{4} x \sqrt{-g}\left[\frac{R}{4}-\frac{1}{4} F^{\mu \nu} F_{\mu \nu}\right]
$$

where we use natural units $c=G=\hbar$ such that $4 \pi \epsilon_{0}=1$. Therefore, the scalar field is a test field, whose backreaction on the spacetime is neglected. Moreover, we consider a non-minimal coupling between the scalar and the vector field; in particular, as in the previous chapter, we study a system with a gauge ChernSimons coupling

$$
g_{F} \phi \tilde{F}^{\mu \nu} F_{\mu \nu},
$$

where $g_{F}$ is a dimensionless coupling constant (it has been weighted with appropriate powers of the Planck mass) and

$$
\tilde{F}_{\mu \nu}=\epsilon_{\mu \nu \alpha \beta} F^{\alpha \beta}
$$

as usual. Being the geometry spherically symmetric, the gravity Chern-Simons term

$$
g_{R} \phi \tilde{R}_{\mu \nu \alpha \beta} R^{\mu \nu \alpha \beta}
$$

vanishes. Hence, the full action reads:

$$
S=\int d^{4} x \sqrt{-g}\left[\frac{R}{4}-\frac{1}{4} F^{\mu \nu} F_{\mu \nu}-\frac{1}{2} \partial_{\mu} \phi \partial^{\mu} \phi-\frac{g_{F}}{8} \phi F^{\mu \nu} \tilde{F}_{\mu \nu}\right] .
$$

The Einstein's Equation, the vector's equation and the scalar field's equation respectively are:

$$
\begin{gathered}
R_{\mu \nu}-\frac{1}{2} R g_{\mu \nu}=2\left(T_{\mu \nu}^{\phi}+T_{\mu \nu}^{F}\right), \\
\nabla_{\alpha}\left(\partial^{\alpha} A_{\mu}-\partial_{\mu} A^{\alpha}\right)-g_{F} \epsilon_{\mu \rho \alpha \beta} \partial^{\alpha} \phi \partial^{\beta} A^{\rho}=0, \\
\square \phi=\frac{1}{4}\left(g_{F} F_{\alpha \beta} \tilde{F}^{\alpha \beta}\right),
\end{gathered}
$$




\section{BLACK HOLE PERTURBATIONS}

where the energy-stress-momentum tensors are defined as:

$$
\begin{aligned}
& T_{\mu \nu}^{\phi}=\partial_{\mu} \phi \partial_{\nu} \phi-\frac{1}{2} g_{\mu \nu} \partial^{\alpha} \phi \partial_{\alpha} \phi \\
& T_{\mu \nu}^{F}=F_{\mu}{ }^{\alpha} F_{\nu \alpha}-\frac{1}{4} g_{\mu \nu} F^{\alpha \beta} F_{\alpha \beta} .
\end{aligned}
$$

As it can be noticed from (5.8), the Chern-Simons coupling does not appear in the Einstein's equation. This fact was expected, since the Chern-Simons term (5.3]) is a total derivative with respect to the metric; on the contrary, both the vector and the scalar equation depend on the Chern-Simons coupling. As discussed in the previous section, motivated by GWs detections there is currently great interest about black holes QNMs and surrounding scalar fields, whose QNMs could affect the ringdown frequencies at the end of the merger phase. For this reason, we now study the black hole perturbations, the vector and the scalar ones for the system described by the action (5.6) .

The linear perturbations of the gravity, the vector and the scalar field are respectively defined as:

$$
\begin{aligned}
& g_{\mu \nu} \rightarrow \bar{g}_{\mu \nu}+h_{\mu \nu}, \\
& A_{\mu} \rightarrow \bar{A}_{\mu}+a_{\mu}, \\
& \phi \rightarrow \bar{\phi}+\delta \phi,
\end{aligned}
$$

where the barred quantities denote the background solutions.

In the scenario we are considering, since the scalar field is a test field and it does not backreact with the geometry, the background axion field $\bar{\phi}$ vanishes. However, to keep equations as more general as possible, we will keep the background fields unspecified until the explicit solutions will be determined. In the following, to avoid an unnecessary heavy notation, we will omit the bars, and we will denote simply with $g, A$ and $\phi$ the background solutions. At linear order in perturbations, 
the Einstein's equation reads:

$$
\begin{aligned}
& +h_{\mu \nu}\left[R-2 \nabla_{\gamma} \phi \nabla^{\gamma} \phi+2\left(\nabla_{\gamma} A_{\delta}-\nabla_{\delta} A_{\gamma}\right) \nabla^{\delta} A^{\gamma}\right] \\
& -4 h_{\gamma \delta}\left[\nabla_{\mu} A^{\gamma} \nabla_{\mu} A^{\delta}-\nabla_{\mu} A^{\delta} \nabla^{\gamma} A_{\nu}+\nabla^{\gamma} A_{\mu}\left(-\nabla_{\nu} A^{\delta}+\nabla^{\delta} A_{\nu}\right)\right] \\
& +4\left(\nabla_{\nu} a_{\mu}-\nabla_{\gamma} a_{\nu}\right)\left(\nabla_{\mu} A^{\gamma}-\nabla^{\gamma} A_{\mu}\right)+4\left(\nabla_{\mu} a_{\gamma}-\nabla_{\gamma} a_{\mu}\right)\left(\nabla_{\nu} A^{\gamma}-\nabla^{\gamma} A_{\nu}\right) \\
& +4 \nabla_{\mu} \delta \phi \nabla_{\nu} \phi+4 \nabla_{\mu} \phi \nabla_{\nu} \delta \phi+\nabla_{\nu} \nabla_{\mu} h_{\gamma}^{\gamma}-\nabla_{\gamma} \nabla_{\mu} h_{\nu}^{\gamma}-\nabla_{\gamma} \nabla_{\nu} h_{\mu}^{\gamma}+\nabla_{\gamma} \nabla^{\gamma} h_{\mu \nu} \\
& -g_{\mu \nu}\left\{h^{\gamma \delta} R_{\gamma \delta}-\nabla_{\delta} \nabla_{\gamma} h^{\gamma \delta}+\nabla_{\delta} \nabla^{\delta} h_{\gamma}^{\gamma}+2 \nabla^{\gamma} \phi\left(2 \nabla_{\gamma} \delta \phi-h_{\gamma \delta} \nabla^{\delta} \phi\right)\right. \\
& \left.\quad-2 \nabla^{\delta} A^{\gamma}\left[2 \nabla_{\gamma} a_{\delta}-2 \nabla_{\delta} a_{\gamma}+h_{\delta \eta} \nabla^{\eta} A_{\gamma}+h_{\gamma \eta}\left(\nabla_{\delta} A^{\eta}-2 \nabla^{\eta} A_{\delta}\right)\right]\right\}=0 .
\end{aligned}
$$

Instead, the vector equation (5.9) perturbed at linear order is:

$$
\begin{aligned}
& h_{\gamma}^{\gamma}\left(-\nabla_{\nu} \nabla_{\mu} A^{\nu}+\nabla_{\nu} \nabla^{\nu} A_{\mu}\right)-\left(\nabla_{\mu} A^{\nu}-\nabla^{\nu} A_{\mu}\right)\left(\nabla_{\nu} h_{\gamma}^{\gamma}-2 \nabla_{\gamma} h_{\nu}^{\gamma}\right) \\
& -2\left\{\nabla_{\nu} \nabla_{\mu} a^{\nu}-\nabla_{\nu} \nabla^{\nu} a_{\mu}-\nabla_{\nu} h_{\mu \gamma} \nabla^{\gamma} A^{\nu}+\nabla_{\gamma} h_{\mu \nu} \nabla^{\gamma} A^{\nu}+h_{\nu \gamma}\left(-\nabla^{\gamma} \nabla_{\mu} A^{\nu}+\nabla^{\gamma} \nabla^{\nu} A_{\mu}\right)\right. \\
& \left.\quad+g_{F}\left[\left(\epsilon_{\mu \gamma \delta \eta} h_{\nu}^{\eta}-\epsilon_{\mu \nu \delta \eta} h_{\gamma}^{\eta}+\epsilon_{\mu \nu \gamma \eta} h_{\delta}^{\eta}-\epsilon_{\mu \nu \gamma \delta} h_{\eta}^{\eta}\right) \nabla^{\delta} \phi-\epsilon_{\mu \nu \gamma \delta} \nabla^{\gamma} A^{\nu} \nabla^{\delta} \delta \phi\right]\right\}=0 .
\end{aligned}
$$

At the end, perturbing the scalar equation (5.9) up to linear order we get:

$+\nabla_{\mu} \nabla^{\mu} \delta \phi+\nabla^{\mu} \phi\left(\nabla_{\mu} h_{\nu}^{\nu}-2 \nabla_{\nu} h_{\mu}^{\nu}\right)-2 h^{\mu \nu} \nabla_{\nu} \nabla_{\mu} \phi+h_{\mu}^{\mu} \nabla_{\nu} \nabla^{\nu} \phi$
$+g_{F} \nabla^{\nu} A^{\mu}\left[-2 \epsilon_{\nu \gamma \delta \eta} h_{\mu}^{\eta} \nabla^{\delta} A^{\gamma}+2 \epsilon_{\mu \gamma \delta \eta} h_{\nu}^{\eta} \nabla^{\delta} A^{\gamma}-\epsilon_{\mu \nu \gamma \delta}\left(h_{\eta}^{\eta} \nabla^{\delta} A^{\gamma}+2 \nabla^{\delta} a^{\gamma}\right)\right]=0$.

\subsection{Regge-Wheeler gauge}

Since we want to study perturbations around a spherically symmetric black hole solution, we use the following generic ansatz for a spherically symmetric background metric:

$$
g_{\mu \nu}=\left(\begin{array}{cccc}
-F(r) & 0 & 0 & 0 \\
0 & G(r) & 0 & 0 \\
0 & 0 & r^{2} & 0 \\
0 & 0 & 0 & r^{2} \sin ^{2} \theta
\end{array}\right) \text {, }
$$




\section{BLACK HOLE PERTURBATIONS}

where the standard Schwarzschild case is obtained with

$$
\begin{aligned}
& G(r)=F^{-1}(r) \\
& F(r)=1-\frac{2 M}{r} .
\end{aligned}
$$

To explicitly compute the perturbations, we expand the metric, the gauge vector and the scalar field perturbations in tensor spherical harmonics $\mathbf{S}_{\mu \nu}^{a}$ (see [377] or [381] for more details), where the index $a$ label each independent component of the basis (before fixing the gauge, there are 10 independent basis components). As the usual scalar spherical harmonics $Y_{l m}$, each tensor spherical harmonic $\mathbf{S}_{\mu \nu}^{a}$ carries the angular indices $(l, m)$, and to be precise we should write the tensor spherical harmonics as $\left(\mathbf{S}_{l m}^{a}\right)_{\mu \nu}$. However, to keep the expressions as easily readable as possible, from now on we will omit the $(l, m)$ indices, with the exception for the cases where the indices $(l, m)$ have an impact on the results. At this point, we exploit the gauge freedom to choose the convenient Regge-Wheeler gauge [367], which reduces the basis tensor harmonics to 6 independent components denoted by labels ( $t t, L 0, R t, T 0, B t, B 1)$ :

$$
\begin{aligned}
h_{\mu \nu}= & +\sum_{l=2}^{\infty} \sum_{m=-l}^{l} h^{B t} \mathbf{S}_{\mu \nu}^{B t}+\sum_{l=1}^{\infty} \sum_{m=-l}^{l} h^{B 1} \mathbf{S}_{\mu \nu}^{B 1}+\sum_{l=0}^{\infty} \sum_{m=-l}^{l}\left[h^{t t} \mathbf{S}_{\mu \nu}^{t t}+h^{L 0} \mathbf{S}_{\mu \nu}^{L 0}\right] \\
& +\sum_{l=1}^{\infty} \sum_{m=-l}^{l} h^{R t} \mathbf{S}_{\mu \nu}^{R t}+\sum_{l=2}^{\infty} \sum_{m=-l}^{l} h^{T 0} \mathbf{S}_{\mu \nu}^{T 0} .
\end{aligned}
$$

Adopting Maggiore's notation [377], it is convenient to redefine the perturbation functions $h^{a}$ in the following way:

$$
\begin{aligned}
& h^{t t}(t, r)=F(r) H_{0}(t, r) \\
& h^{L 0}(t, r)=G(r) H_{2}(t, r), \\
& h^{T 0}(t, r)=r^{2} K(t, r), \\
& h^{R t}(t, r)=H_{1}(t, r), \\
& h^{B t}(t, r)=-h_{0}(t, r), \\
& h^{B 1}(t, r)=-h_{1}(t, r) .
\end{aligned}
$$


With these redefinitions, writing explicitly the angular terms the full metric's expansion reads:

$$
h_{\mu \nu}=\left(\begin{array}{cccc}
F(r) H_{0} & H_{1} & -h_{0} \sin \theta^{-1} \partial_{\varphi} & h_{0} \sin \theta \partial_{\theta} \\
H_{1} & G(r) H_{2} & -h_{1} \sin \theta^{-1} \partial_{\varphi} & h_{1} \sin \theta \partial_{\theta} \\
-h_{0} \sin \theta^{-1} \partial_{\varphi} & -h_{1} \sin \theta^{-1} \partial_{\varphi} & K r^{2} & 0 \\
h_{0} \sin \theta \partial_{\theta} & h_{1} \sin \theta \partial_{\theta} & 0 & K r^{2} \sin ^{2} \theta
\end{array}\right) Y(\theta, \varphi) .
$$

where we have omitted the $(t, r)$ dependence of the perturbation functions.

In the same way, we now need to expand the vector field $A_{\mu}$ in terms of vector spherical harmonics (the full derivation can be found in [377]), and we get:

$$
a^{\mu}=\left(\begin{array}{c}
a_{0}(t, r) \\
a_{R}(t, r) \\
a_{B}(t, r) \sin \theta^{-1} \partial_{\varphi}+a_{E}(t, r) \partial_{\theta} \\
a_{E}(t, r) \partial_{\varphi}-a_{B}(t, r) \sin \theta \partial_{\theta}
\end{array}\right) Y(\theta, \varphi)
$$

Finally, we also have to expand the scalar field's perturbations; since those are scalar perturbations, the angular expansion in simply the usual one with scalar spherical harmonics:

$$
\delta \phi=\delta \phi(t, r) Y(\theta, \varphi)
$$

Under a parity transformation

$$
\theta \rightarrow \pi-\theta, \quad \varphi \rightarrow \pi+\varphi
$$

tensor harmonics which transform as $(-1)^{l}$ are said to be even or polar, while tensor harmonics transforming as $(-1)^{l+1}$ are said to be odd or axial. Therefore, we extend the definition of polar/axial to the scalar functions associated with the tensor harmonics, thus we have the functions $\left(H_{0}, H_{1}, H_{2}, a_{0}, a_{R}, a_{E}, \delta \phi\right)$ being polar and the functions $\left(h_{0}, h_{1}, a_{B}\right)$ being axial. Since the Schwarzschild background does not mix parity odd and parity even perturbations, it is convenient to split the metric perturbations into an odd (axial) and an even (polar) sector:

$$
h_{\mu \nu}=h_{\mu \nu}^{a x}+h_{\mu \nu}^{p o l},
$$


with

$$
\begin{aligned}
h_{\mu \nu}^{a x}= & -\sum_{l=2}^{\infty} \sum_{m=-l}^{l} h_{0} \mathbf{S}_{\mu \nu}^{B t}-\sum_{l=1}^{\infty} \sum_{m=-l}^{l} h_{1} \mathbf{S}_{\mu \nu}^{B 1} \\
h_{\mu \nu}^{p o l}= & +\sum_{l=0}^{\infty} \sum_{m=-l}^{l}\left[F(r) H_{0}(t, r) \mathbf{S}_{\mu \nu}^{t t}+G(r) H_{2}(t, r) \mathbf{S}_{\mu \nu}^{L 0}\right] \\
& +\sum_{l=1}^{\infty} \sum_{m=-l}^{l}\left[H_{1}(t, r) \mathbf{S}_{\mu \nu}^{R t}+r^{2} K(t, r) \mathbf{S}_{\mu \nu}^{T 0}\right] .
\end{aligned}
$$

In the same way, we also decompose the generic energy-stress-momentum tensor $T_{\mu \nu}$ :

$$
T_{\mu \nu}=T_{\mu \nu}^{a x}+T_{\mu \nu}^{p o l}
$$

with

$$
\begin{aligned}
T_{\mu \nu}^{a x}= & +\sum_{l=2}^{\infty} \sum_{m=-l}^{l} s^{B 2} \mathbf{S}_{\mu \nu}^{B 2}+\sum_{l=1}^{\infty} \sum_{m=-l}^{l}\left[s^{B t} \mathbf{S}_{\mu \nu}^{B t}+s^{B 1} \mathbf{S}_{\mu \nu}^{B 1}\right] \\
T_{\mu \nu}^{p o l}= & +\sum_{l=0}^{\infty} \sum_{m=-l}^{l}\left[s^{t t} \mathbf{S}_{\mu \nu}^{t t}+s^{R t} \mathbf{S}_{\mu \nu}^{R t}+s^{L 0} \mathbf{S}_{\mu \nu}^{L 0}+s^{T 0} \mathbf{S}_{\mu \nu}^{T 0}\right] \\
& +\sum_{l=1}^{\infty} \sum_{m=-l}^{l}\left[s^{E t} \mathbf{S}_{\mu \nu}^{E t}+s^{E 1} \mathbf{S}_{\mu \nu}^{E 1}\right]+\sum_{l=2}^{\infty} \sum_{m=-l}^{l} s^{E 2} \mathbf{S}_{\mu \nu}^{E 2}
\end{aligned}
$$

In the following, we will discuss Schwarzschild, Reissner-Nordström and ChernSimons perturbations in the RW gauge. 


\subsubsection{Schwarzschild case}

To have a reference point, we now discuss the metric's perturbations equation for the Schwarzschild case, where the metric is:

$$
d s^{2}=-F(r) d t^{2}+\frac{1}{F(r)} d r^{2}+r^{2} d \theta^{2}+r^{2} \sin ^{2} \theta d \varphi^{2}
$$

with $F(r)=1-\frac{2 M}{r}$.

From the perturbed Einstein's Equation (5.12), using the explicit expansion of the perturbations we get:

$$
\left\{F\left[\frac{2}{r} \partial_{t} h_{1}+\partial_{t} \partial_{r} h_{1}-\partial_{r}^{2} h_{0}\right]+h_{0}\left[F^{\prime \prime}+\frac{2 F^{\prime}}{r}+\frac{2 F-2}{r^{2}}\right]\right\} \sin \theta \partial_{\theta} Y(\theta, \varphi)
$$

$(t \varphi)$ :

$$
\begin{aligned}
& -\frac{h_{0}}{r^{2}} \sin \theta \partial_{\theta}\left[\frac{1}{\sin ^{2} \theta} \partial_{\theta}^{2} Y(\theta, \varphi)+\frac{\cos \theta}{\sin \theta} \partial_{\theta} Y(\theta, \varphi)+\partial_{\varphi}^{2} Y(\theta, \varphi)\right] \\
& +\left\{F^{\prime} H_{1}+F \partial_{r} H_{1}-\partial_{t} H_{2}-\partial_{t} K\right\} \partial_{\varphi} Y(\theta, \varphi)=0,
\end{aligned}
$$$$
\left\{\frac{1}{F}\left[\partial_{t}^{2} h_{1}-\partial_{r} \partial_{t} h_{0}+\frac{2}{r} \partial_{t} h_{0}\right]+h_{1}\left[F^{\prime \prime}+\frac{2 F^{\prime}}{r}-\frac{2}{r^{2}}\right]\right\} \sin \theta \partial_{\theta} Y(\theta, \varphi)
$$

$(r \varphi): \quad-\frac{h_{1}}{r^{2}} \sin \theta \partial_{\theta}\left[\frac{1}{\sin ^{2} \theta} \partial_{\theta}^{2} Y(\theta, \varphi)+\frac{\cos \theta}{\sin \theta} \partial_{\theta} Y(\theta, \varphi)+\partial_{\varphi}^{2} Y(\theta, \varphi)\right]$

$(\theta \varphi)$

$$
+\left\{\frac{\left[H_{0}-H_{2}\right]\left[r F^{\prime}-2 F\right]+2 F \partial_{r}\left[H_{0}-K\right]-2 r \partial_{t} H_{1}}{2 r F}\right\} \partial_{\varphi} Y(\theta, \varphi)=0,
$$

$$
\frac{\partial_{t} h_{0}-F F^{\prime} h_{1}-F^{2} \partial_{r} h_{1}}{F}\left[\frac{1}{\sin ^{2} \theta} \partial_{\varphi}^{2} Y(\theta, \varphi)+\frac{\cos \theta}{\sin \theta} \partial_{\theta} Y(\theta, \varphi)+\partial_{\theta}^{2} Y(\theta, \varphi)\right] \sin \theta
$$

$$
+\left[H_{0}-H_{2}\right]\left[\frac{1}{\sin ^{2} \theta} \partial_{\varphi} Y(\theta, \varphi)-\partial_{\varphi} \partial_{\theta} Y(\theta, \varphi)\right]=0
$$

$(t t):$

$$
\left\{\frac{r^{2} F^{\prime}}{2 F} \partial_{r} K+\frac{K}{F}-\frac{H_{0}}{F}\left[-1+F+r F^{\prime}\right]-H_{2}+r \partial_{r}\left(H_{2}-3 K\right)-r^{2} \partial_{r}^{2} K\right\} Y(\theta, \varphi)
$$

$$
+\frac{1}{2 F}\left[H_{2}+K\right]\left[\frac{1}{\sin ^{2} \theta} \partial_{\theta}^{2} Y(\theta, \varphi)+\frac{\cos \theta}{\sin \theta} \partial_{\theta} Y(\theta, \varphi)+\partial_{\varphi}^{2} Y(\theta, \varphi)\right]=0,
$$


$(r r):\left\{2\left[H_{2}-K\right]+r F \partial_{r}\left[H_{0}-K\right]-r^{2} F^{\prime} \partial_{r} K-4 \partial_{t} H_{1}+\frac{2 r^{2}}{F} \partial_{t}^{2} K\right\} Y(\theta, \varphi)$

$(\theta \theta):$

$$
+\left[H_{0}-K\right]\left[\frac{1}{\sin ^{2} \theta} \partial_{\theta}^{2} Y(\theta, \varphi)+\frac{\cos \theta}{\sin \theta} \partial_{\theta} Y(\theta, \varphi)+\partial_{\varphi}^{2} Y(\theta, \varphi)\right]=0,
$$

$$
+\frac{2}{F}\left[F F^{\prime} h_{1}+F^{2} \partial_{r} h_{1}-\partial_{t} h_{0}\right] \frac{\partial_{\varphi}}{\sin \theta}\left[Y(\theta, \varphi)-\partial_{\theta} Y(\theta, \varphi)\right]
$$

$$
\begin{aligned}
& -r\left\{F\left[\partial_{r} H_{0}+\partial_{r} H_{2}-2 \partial_{r} K+r \partial_{r}^{2} H_{0}-r \partial_{r}^{2} K\right]+\frac{r}{F}\left[+\partial_{t}^{2} H_{2}+\partial_{t}^{2} K-F^{\prime} \partial_{t} H_{1}\right]\right. \\
& \left.-\left[\left(H_{2}-K\right)\left(2 F^{\prime}+r F^{\prime \prime}\right)+\frac{r F^{\prime}}{2} \partial_{r}\left(3 H_{0}+H_{2}-K\right)-2 \partial_{t}\left(H_{1}-r \partial_{r} H_{1}\right)\right]\right\} Y(\theta, \varphi) \\
& +\left[H_{2}-H_{0}\right]\left[\frac{1}{\sin ^{2} \theta} \partial_{\varphi}^{2} Y(\theta, \varphi)+\frac{\cos \theta}{\sin \theta} \partial_{\theta} Y(\theta, \varphi)\right]=0,
\end{aligned}
$$

$$
+\frac{2}{F}\left[F F^{\prime} h_{1}+F^{2} \partial_{r} h_{1}-\partial_{t} h_{0}\right] \frac{\partial_{\varphi}}{\sin \theta}\left[Y(\theta, \varphi)-\partial_{\theta} Y(\theta, \varphi)\right]
$$

$$
+r\left\{F\left[\partial_{r} H_{0}+\partial_{r} H_{2}-2 \partial_{r} K+r \partial_{r}^{2} H_{0}-r \partial_{r}^{2} K\right]+r\left[\partial_{t}^{2} K-F^{\prime} \partial_{t} H_{1}+\partial_{t}^{2} H_{2}\right]\right.
$$

$\left.+\left[\left(H_{2}-K\right)\left(2 F^{\prime}+r F^{\prime \prime}\right)+\frac{r}{2} F^{\prime} \partial_{r}\left(3 H_{0}+H_{2}-2 K\right)-2 \partial_{t}\left(H_{1}+r \partial_{r} H_{1}\right)\right]\right\} Y(\theta, \varphi)$

$+F\left[H_{0}-H_{2}\right] \partial_{\theta}^{2} Y(\theta, \varphi)=0$,

$(t r):$

$$
\left\{F H_{1}+r^{2} \frac{F^{\prime}}{2 F} \partial_{t} K+r H_{2}-\partial_{t} K-r^{2} \partial_{r} \partial_{t} K-\frac{H_{1}}{2}\left[-1+r F^{\prime}\right]\right\} Y(\theta, \varphi)
$$

$$
\begin{aligned}
& -\frac{H_{1}}{2}\left[\frac{1}{\sin ^{2} \theta} \partial_{\theta}^{2} Y(\theta, \varphi)+\frac{\cos \theta}{\sin \theta} \partial_{\theta} Y(\theta, \varphi)+\partial_{\varphi}^{2} Y(\theta, \varphi)\right]=0, \\
& \left\{F\left[\partial_{r}^{2} h_{0}-\frac{2}{r} \partial_{t} h_{1}-\partial_{t} \partial_{r} h_{1}\right]+h_{0}\left[2-2 F-\partial_{r}\left(r^{2} F^{\prime}\right)\right]\right\} \partial_{\varphi} Y(\theta, \varphi)
\end{aligned}
$$

$(t \theta): \quad+\frac{h_{0}}{r^{2}} \partial_{\varphi}\left[\frac{1}{\sin ^{2} \theta} \partial_{\theta}^{2} Y(\theta, \varphi)+\frac{\cos \theta}{\sin \theta} \partial_{\theta} Y(\theta, \varphi)+\partial_{\varphi}^{2} Y(\theta, \varphi)\right]$

$$
+\left\{\partial_{r}\left[F H_{1}\right]-\partial_{t}\left[H_{2}+K\right]\right\} \sin \theta \partial_{\theta} Y(\theta, \varphi)=0,
$$




$$
\begin{aligned}
& \left\{F h_{1}\left[2-2 r F^{\prime}-r^{2} F^{\prime \prime}\right]-\partial_{t}^{2} h_{1}+\partial_{t} \partial_{r} h_{0}-\frac{2}{r} \partial_{t} h_{0}\right\} \partial_{\varphi} Y(\theta, \varphi) \\
(r \theta): \quad & +F h_{1} \partial_{\varphi}\left[\frac{1}{\sin ^{2} \theta} \partial_{\theta}^{2} Y+\frac{\cos \theta}{\sin \theta} \partial_{\theta} Y(\theta, \varphi)+\partial_{\varphi}^{2} Y(\theta, \varphi)\right] \\
& +\frac{r F^{\prime}\left[H_{0}+H_{2}\right]+2 F\left[H_{2}-H_{0}+r \partial_{r} H_{0}-r \partial_{r} K\right]-2 r \partial_{t} H_{1}}{2 r} \sin \theta \partial_{\theta} Y(\theta, \varphi),
\end{aligned}
$$

Looking at the previous equations, we notice that it is possible to separate each equation into an axial and a polar part, namely into two equations containing only axial/polar functions. In fact, from $(5.28-5.37)$ we observe that the angular dependence of the axial and the polar parts are mutually orthogonal (in the sense of spherical harmonics orthonormality), and it is therefore possible to solve the two equations (axial and polar) separately. So, this justifies the statement that the Schwarzschild background decouples the axial and the polar perturbations, splitting the perturbations equations into two independent sets of equations, usually referred to as sectors.

\section{$\underline{\text { AXIAL SECTOR }}$}

We start looking at the axial sector of the metric's perturbations. Since we can split each equation into an axial and a polar part which must be independently satisfied, here we work only on the axial parts.

From the equations ( $5.28-5.37)$, we notice that each axial equation can be divided into a radial and an angular part, and we can therefore proceed with the standard separation of variables $(r, \theta, \varphi)$.

The angular equation is

$$
\left[\frac{1}{\sin ^{2} \theta} \partial_{\varphi}^{2} Y(\theta, \varphi)+\frac{\cos \theta}{\sin \theta} \partial_{\theta} Y(\theta, \varphi)+\partial_{\theta}^{2} Y(\theta, \varphi)\right]+\lambda Y(\theta, \varphi)=0,
$$

which is a General Legendre Equation, whose solution are the spherical harmonics 


\section{BLACK HOLE PERTURBATIONS}

$Y_{l m}(\theta, \varphi)$ and the separation constant is

$$
\lambda=l(l+1)
$$

For the axial sector, we can reduce the 10 equations to a set of 3 equations (see $[3 / 7])$ :

$$
\begin{aligned}
& \partial_{r}^{2} h_{0}-\left(\partial_{r}+\frac{2}{r}\right) \partial_{0} h_{1}+\frac{1}{F}\left[\frac{2}{r} \frac{d F}{d r}-\frac{l(l+1)}{r^{2}}\right] h_{0}=+4 s_{l m}^{B t} \\
& \partial_{0}^{2} h_{1}-\left(\partial_{r}-\frac{2}{r}\right) \partial_{0} h_{0}+F \frac{(l-1)(l+2)}{r^{2}} h_{1}=-4 F s_{l m}^{B 1} \\
& \frac{1}{F} \partial_{0} h_{0}-\partial_{r}\left(F h_{1}\right)=-4 s_{l m}^{B 2}
\end{aligned}
$$

where the first two equations hold for $l \geq 1$ and the last one holds for $l \geq 2$.

For more generality, in $(5.40)$ we have included on the RHSs the source terms $\left(s_{l m}^{B t}, s_{l m}^{B 1}, s_{l m}^{B 2}\right)$, although the equations $(5.28-5.37)$ correspond to the vacuum case, with the sources being: $s_{l m}^{B t}=s_{l m}^{B 1}=s_{l m}^{B 2}=0$. Looking at (5.40), we have three equations relating two metric functions $\left(h_{0}, h_{1}\right)$, which means that the equations are not independent and whose consistency is ensured by Bianchi identities and by the stress-energy tensor conservation. Introducing the Regge-Wheeler function

$$
Q_{l m}(t, r)=\frac{1}{r} F(r) h_{1}(t, r),
$$

it is possible to reduce (details can be found on [377]) the set (5.40) to a single differential equation for the function $Q_{l m}(t, r)$. After introducing the radial tortoise coordinate $r_{*}$ defined by

$$
\partial_{*}=\frac{\partial}{\partial r_{*}}=F(r) \frac{\partial}{\partial r}=F(r) \partial_{r}
$$

we arrive at a single differential equation:

$$
\left(\partial_{*}^{2}-\partial_{0}^{2}\right) Q_{l m}(t, r)-V_{l}^{\mathrm{RW}}(r) Q_{l m}(t, r)=S_{l m}^{\mathrm{ax}}(t, r),
$$

with potential

$$
V_{l}^{\mathrm{RW}}(r)=F(r)\left[\frac{l(l+1)}{r^{2}}-\frac{6 M}{r^{3}}\right]
$$


and source term

$$
S_{l m}^{\mathrm{ax}}(t, r)=4 \frac{F(r)}{r}\left\{F(r) s_{l m}^{B 1}(t, r)+\left(\partial_{r}-\frac{2}{r}\right)\left[F(r) s_{l m}^{B 2}(t, r)\right]\right\} .
$$

The equation (5.43) is commonly known as Regge-Wheeler equation and the potential $V(r)$ is the Regge-Wheeler potential. Remarkably, the form of the ReggeWheeler potential remains almost identical in the cases of vector and scalar perturbations:

$$
V_{l}^{\mathrm{RW}}(r)=F(r)\left[\frac{l(l+1)}{r^{2}}+\frac{3\left(1-\mathrm{s}^{2}\right) r_{S}}{r^{3}}\right],
$$

where $\mathrm{s}=2,1,0$ for gravitational, electromagnetic and scalar perturbations respectively and $r_{S}$ is the Schwarzschild radius: $r_{S}=2 M$. Alternatively, after a Fourier transform $\left(\partial_{t}=-i \omega\right)$ we can recast the equation (5.43) as a Schrödingerlike equation

$$
\partial_{*}^{2} \tilde{Q}_{l m}(\omega, r)+\left[\omega^{2}-V_{l}^{\mathrm{RW}}(r)\right] \tilde{Q}_{l m}(\omega, r)=\tilde{S}_{l m}^{\mathrm{ax}}(\omega, r) .
$$

\section{POLAR SECTOR}

In the polar sector, the Einstein's Equations (5.28-5.37) lead to a set of 7 equations [377]:

$$
\begin{aligned}
& F^{2} \partial_{r}^{2} K+\frac{F}{r}\left(3-\frac{5 M}{r}\right) \partial_{r} K-\frac{F^{2}}{r} \partial_{r} H_{2}+\frac{F}{r^{2}}\left(K-H_{2}\right)-F \frac{l(l+1)}{2 r^{2}}\left(K+H_{2}\right)=-s^{t t}, \\
& \partial_{t}\left[\partial_{r} K+\frac{1}{r}\left(K-H_{2}\right)-\frac{M}{r^{2} F} K\right]-\frac{l(l+1)}{2 r^{2}} H_{1}=-s^{R t}, \\
& \frac{\partial_{t}^{2} K}{F^{2}}-\frac{r-M}{r^{2} F} \partial_{r} K-\frac{2 \partial_{t} H_{1}}{r F}+\frac{\partial_{r} H_{0}}{r}-\frac{K-H_{2}}{r^{2} F}+\frac{l(l+1)}{2 r^{2} F}\left(K-H_{0}\right)=-s^{L 0}, \\
& F \partial_{r}^{2}\left(K-H_{0}\right)-\frac{1}{F} \partial_{t}^{2}\left(K+H_{2}\right)+\left(1-\frac{M}{r}\right) \frac{2}{r} \partial_{r} K+2 \partial_{r} \partial_{t} H_{1}+\frac{2}{r F}\left(1-\frac{M}{r}\right) \partial_{t} H_{1} \\
& -\frac{1}{r}\left(1-\frac{M}{r}\right) \partial_{r} H_{2}-\frac{1}{r}\left(1+\frac{M}{r}\right) \partial_{r} H_{0}-\frac{l(l+1)}{2 r^{2}}\left(H_{2}-H_{0}\right)=\frac{4 s^{T 0}}{r^{2}},
\end{aligned}
$$




\section{BLACK HOLE PERTURBATIONS}

which are valid for $l \geq 0$; then we have

$$
\begin{aligned}
& \partial_{r}\left(F H_{1}\right)-\partial_{t}\left(K+H_{2}\right)=4 s^{E t}, \\
& \frac{1}{F} \partial_{t} H_{1}+\partial_{r}\left(K-H_{0}\right)-\frac{2 M}{F r^{2}} H_{0}-\frac{1-M / r}{F r}\left(H_{2}-H_{0}\right)=-4 s^{E 1}
\end{aligned}
$$

which are valid for $l \geq 1$; finally we also have

$$
\left(H_{0}-H_{2}\right)=8 s^{E 2}
$$

which is valid for $l \geq 2$ (we have included the sources $s_{l m}^{i}$ for more generality).

As it happens in the axial sector, the equations above are not independent; after some manipulations (again, details can be found on [377]), we can introduce the Zerilli function $\tilde{Z}_{l m}$ :

$$
\tilde{Z}_{l m}(\omega, r)=\frac{r^{2}}{\lambda r+3 M} \tilde{K}(\omega, r)+\frac{r F(r)}{i \omega(\lambda r+3 M)} \tilde{H}_{1}(\omega, r)
$$

with

$$
\lambda=\frac{(l-1)(l+2)}{2}
$$

and where the tilded functions are the Fourier transforms of the correspondent functions:

$$
\tilde{f}(\omega, r)=\int f(t, r) e^{i \omega t} d t .
$$

With these redefinitions, after introducing the tortoise coordinate $r_{*}$ it is possible to reduce the polar sector equations to a single differential equation for the Zerilli function:

$$
\partial_{*}^{2} \tilde{Z}_{l m}(\omega, r)+\left[\omega^{2}-V_{l}^{Z}(r)\right] \tilde{Z}_{l m}(\omega, r)=\tilde{S}_{l m}^{p o l}(\omega, r),
$$


where

$$
\begin{aligned}
V_{l}^{\mathrm{Z}}(r) & =F(r) \frac{2 \lambda^{2}(\lambda+1) r^{3}+6 \lambda^{2} M r^{2}+18 \lambda M^{2} r+18 M^{3}}{r^{3}(\lambda r+3 M)^{2}}, \\
\tilde{S}_{l m}^{\mathrm{pol}}= & F \partial_{r}\left(F J_{l m}\right)-\frac{4 \tilde{s}_{l m}^{E t}}{i \omega} \frac{F\left[\lambda(\lambda+1) r^{2}+3 M r \lambda+6 M^{2}\right]}{r(\lambda r+3 M)^{2}}-2 \frac{\tilde{s}_{l m}^{R t}}{i \omega} \frac{\lambda r^{2} F^{2}}{(\lambda r+3 M)^{2}} \\
& +2\left(r \tilde{s}_{l m}^{L 0}+2 \tilde{s}_{l m}^{E 1}\right) \frac{r F^{2}}{\lambda r+3 M}-\frac{8 F}{r} \tilde{s}_{l m}^{E 2}
\end{aligned}
$$

and with

$$
J_{l m}=\frac{r}{i \omega(\lambda r+3 M)}\left(r \tilde{s}_{l m}^{R t}+2 \tilde{s}_{l m}^{E t}\right) .
$$

In the next sections, we will study different scenarios including the electromagnetic vector: first, we will study the perturbations of the electric and the magnetic Reissner-Nordström black hole, and then we will investigate the case with a ChernSimons coupling on a Reissner-Nordström background.

\subsection{Electric Reissner-Nordström case}

A few years after the publication of the original Regge-Wheeler paper [18], many authors generalised the purely gravitational perturbations formalism to the case of gravito-electromagnetic ones. In the following, we report a very concise literature review of the most significant works about black hole perturbations in EinsteinMaxwell theory. In particular, we have focused our attention on the most influential results dealing with the Reissner-Nordström geometry, since we are specifically interested in charged, static and spherically symmetric black hole configurations.

- 1974: Zerilli [35] studies gravitational and electromagnetic perturbations of the Reissner-Nordström spacetime. Performing an expansion in tensor and vector spherical harmonics, according to their parity the perturbations can be decoupled into two separate second order Schrödinger equations. 


\section{BLACK HOLE PERTURBATIONS}

- 1974: Moncrief [382, 383] studies both even and odd perturbations of the Reissner-Nordström spacetime, investigating whether the geometry admits unstable modes.

- 1975: Moncrief [352] introduces a gauge invariant approach to study gravitational and electromagnetic perturbations.

- 1981: Xanthopoulos [384] studies the Reissner-Nordström perturbations with an alternative approach, avoiding the traditional distinction between even and odd perturbations.

- 1983: Chandrasekhar [341] publishes a comprehensive monograph on black holes QNMs, with great attention on the charged case.

In the majority of the cited works, the Reissner-Nordström geometry has been considered with solely electric charge, namely with only one non vanishing component of the gauge vector. In this case, authors have found that gravitational and electromagnetic perturbations can be decoupled into an odd and even sector, as it happens in the Schwarzschild case. Moreover, when both the electric and the magnetic charge have been taken into account (such as in [352]), the parity even and the parity odd sectors have been separately studied. In this way, an eventual mixing of parity sectors cannot be spotted, and both gravitational and electromagnetic perturbations lead to Schrödinger-like equations "well contained" into the proper sector, without contributions from opposite parity terms. Continuing our brief chronological literature review, we mention:

- 1988: Kokkotas and Schutz [385] apply WKB techniques to calculate complex frequencies of the Reissner-Nordström QNMs, comparing the results with the numerical integration proposed by Chandrasekhar and Detweiler in [353]. 
- 1990: Leaver [386] introduces a matrix-eigenvalue algorithm based on continued fraction representations method to numerically compute the QNMs of a static, charged black hole.

- 1996: Andersson and Onozawa [387] compute for both slowly and rapidly damped modes the nearly-extremal Reissner-Nordström QNMs .

- 1999: Kokkotas and Schmidt publish an exhaustive review [342] on QNMs including static, rotating, neutral, charged black holes and relativistic stars.

- 2004: Kodama and Ishibashi extend the higher dimensional analysis proposed in [362] to the case of charged black holes [388]; assuming the existence of an electric charge and an electric field surrounding the black hole, the perturbation equations are reduced to two decoupled Schrödinger equations of second order.

- 2005: Natario and Schiappa [38.9] systematically study and classify the QNMs frequencies found in [362, 388], finding that the Reissner-Nordström QNMs frequencies are dimensional dependent.

- 2016: Cardoso et al. [378] show how viable models of minicharged dark matter black holes are described by Reissner-Nordström or Kerr-Newman spacetimes, with an hidden $U(1)$ symmetry playing the role of the usual electromagnetic $U(1)$. Moreover, the authors show that GWs detections can put constraints to the black hole charges, regardless of their nature.

- 2018: Brito and Pacilio [3.90] study the QNMs spectrum of static/slowly rotating black holes with weakly charged Einstein-Maxwell-dilaton coupling. It is found that the system is stable under gravitational, electromagnetic and 
scalar perturbations, with the electromagnetic modes exhibiting "dilatondependent breaking of isospectrality between the axial and polar sectors".

- 2019: Myung and Zou [391] analyse the stability of scalarised, charged black holes in the Einstein-Maxwell-scalar theory. Moreover, in [392] the authors also include a mass term for the scalar field, leading to mass-dependent constraints for the stability of the Reissner-Nordström spacetime.

- 2019: Dotti and Fernandez [393, 394] study the nonmodal linear stability of the outer region of a Reissner-Nordström (A)dS spacetime.

After this short literature review, we can now proceed with a more technical analysis, and we start by studying the metric and the vector perturbations of an electrically charged Reissner-Nordström black hole. As already said, the results we present in this section are known from the Seventies [351, 382], but it is anyway useful to discuss them in order to have a model to be compared to in the next sections.

The electric Reissner-Nordström spacetime is described by the metric

$$
h_{\mu \nu}=\left(\begin{array}{cccc}
-F(r) & 0 & 0 & 0 \\
0 & F(r)^{-1} & 0 & 0 \\
0 & 0 & r^{2} & 0 \\
0 & 0 & 0 & r^{2} \sin ^{2} \theta
\end{array}\right)
$$

with

$$
F(r)=1-\frac{2 M}{r}+\frac{Q^{2}}{r^{2}}
$$

and where the gauge vector reads

$$
A_{\mu}=\left\{-\frac{Q}{r}, 0,0,0\right\}
$$

with $Q$ being the electric charge. 
Since we are now interested in the black hole's perturbations only, we do not consider any external field, and we therefore neglect both the scalar kinetic term and the Chern-Simons coupling in the action ([5.6). So, in this scenario we have

$$
\phi=0, \quad \delta \phi=0
$$

As we have discussed in the previous section, in the Schwarzschild case it is possible to decouple the metric's equation into disconnected axial and polar sectors, and it is therefore possible to separately solve the axial and the polar equations. In the electric Reissner-Nordström case, this separability still holds (see for example the original works [351, 382, 38:3]), and hence we can choose to focus our attention on the axial sector only. As in the Schwarzschild case, from the components $(t \varphi),(r \varphi)$ and $(\theta \varphi)$ of the perturbed Einstein's Equations we can obtain the axial equations:

$$
\begin{aligned}
& (t \varphi):\left\{F\left[\frac{2}{r} \partial_{t} h_{1}+\partial_{t} \partial_{r} h_{1}-\partial_{r}^{2} h_{0}\right]+h_{0}\left[F^{\prime \prime}+\frac{2 F^{\prime}}{r}+\frac{2 F-2}{r^{2}}\right]+4 F A_{0}^{\prime} \partial_{r} a_{B}\right. \\
& \left.-2\left(A_{0}^{\prime}\right)^{2} h_{0}\right\} S_{\theta} Y(\theta, \varphi)-\frac{h_{0}}{r^{2}} S_{\theta}\left[\frac{1}{\sin ^{2} \theta} \partial_{\theta}^{2} Y(\theta, \varphi)+\frac{\cos \theta}{\sin \theta} \partial_{\theta} Y(\theta, \varphi)+\partial_{\varphi}^{2} Y(\theta, \varphi)\right] \\
& +\left\{F^{\prime} H_{1}+F \partial_{r} H_{1}-\partial_{t} H_{0}-\partial_{t} K+4 F A_{0}^{\prime}\left[a_{R}-\partial_{r} a_{E}\right]\right\} \partial_{\varphi} Y(\theta, \varphi)=0, \\
& (r \varphi):\left\{\frac{1}{F}\left[\partial_{t}^{2} h_{1}-\partial_{r} \partial_{t} h_{0}+\frac{2}{r} \partial_{t} h_{0}\right]+h_{1}\left[F^{\prime \prime}+2 \partial_{r} \frac{F}{r}\right]+4 \frac{A_{0}^{\prime}}{F} \partial_{t} a_{B}\right. \\
& \left.-2\left(A_{0}^{\prime}\right)^{2} h_{1}\right\} S_{\theta} Y(\theta, \varphi)-\frac{h_{1}}{r^{2}} S_{\theta}\left[\frac{1}{\sin ^{2} \theta} \partial_{\theta}^{2} Y(\theta, \varphi)+\frac{\cos \theta}{\sin \theta} \partial_{\theta} Y(\theta, \varphi)+\partial_{\varphi}^{2} Y(\theta, \varphi)\right] \\
& +\frac{1}{F}\left\{F^{\prime} H_{0}+F \partial_{r} H_{0}-F \partial_{r} K-\partial_{t} H_{1}+4 A_{0}^{\prime}\left[a_{0}-\partial_{t} a_{E}\right]\right\} \partial_{\varphi} Y(\theta, \varphi)=0, \\
& (\theta \varphi): \frac{\partial_{t} h_{0}-F F^{\prime} h_{1}-F^{2} \partial_{r} h_{1}}{F}\left[\frac{1}{\sin ^{2} \theta} \partial_{\varphi}^{2} Y(\theta, \varphi)+\frac{\cos \theta}{\sin \theta} \partial_{\theta} Y(\theta, \varphi)+\partial_{\theta}^{2} Y(\theta, \varphi)\right] \sin \theta \\
& +\left[H_{0}-H_{2}\right]\left[\frac{1}{\sin ^{2} \theta} \partial_{\varphi} Y(\theta, \varphi)-\partial_{\varphi} \partial_{\theta} Y(\theta, \varphi)\right]=0 .
\end{aligned}
$$

where we have written $S_{\theta}=\sin \theta \partial_{\theta}$ for brevity.

From these equations, we notice that the axial sector $\left(h_{0}, h_{1}, a_{B}\right)$ is orthogonal (in the sense of the spherical harmonics orthonormality) to the polar one, as expected. 
Moreover, from the $\theta$ component of the perturbed vector equation (5.13) we get:

$$
\begin{aligned}
A_{\theta}: & F^{2} r^{2} \partial_{r}^{2} a_{B}-r^{2} \partial_{t}^{2} a_{B}-F\left[-r^{2} F^{\prime} \partial_{r} a_{B}\right]-F r^{2} A_{0}^{\prime}\left[-\frac{2}{r} h_{0}+\partial_{r} h_{0}-\partial_{t} h_{1}\right] \\
& +F a_{B}\left[\frac{1}{\sin ^{2} \theta} \partial_{\varphi}^{2} Y(\theta, \varphi)+\frac{\cos \theta}{\sin \theta} \partial_{\theta} Y(\theta, \varphi)+\partial_{\theta}^{2} Y(\theta, \varphi)\right]=0 .
\end{aligned}
$$

Again, due to spherical harmonics orthonormality we can decouple the previous equation into an axial and a polar one, which can be solved separately. Due to this, it is evident that axial functions are decoupled from the polar ones, and therefore there is no parity mixing. Looking at the axial equation, after performing a separation of variables we have to solve the angular equation. With a bit of further manipulation, introducing the tortoise derivative

$$
\partial_{*}=F(r) \partial_{r}
$$

to reduce the set (5.60) to a single master equation, and then moving to the Fourier space $\left(\partial_{t}=-i \omega\right)$ to recast the axial equations in the in terms of just 2 axial functions $\tilde{W}(\omega, r)$ and $\tilde{a}_{B}(\omega, r)$ we arrive at:

$$
\begin{aligned}
& \partial_{*}^{2} \tilde{W}(\omega, r)+\omega^{2} \tilde{W}(\omega, r)-F\left[\frac{l(l+1)}{r^{2}}-\frac{6 M}{r^{3}}+\frac{4 Q^{2}}{r^{4}}\right] \tilde{W}(\omega, r)=-4 i \omega \frac{F A_{0}^{\prime}}{r} \tilde{a}_{B}(\omega, r), \\
& \partial_{*}^{2} \tilde{a}_{B}(\omega, r)+\omega^{2} \tilde{a}_{B}(\omega, r)-F\left[\frac{l(l+1)}{r^{2}}+\frac{4 Q^{2}}{r^{4}}\right] \tilde{a}_{B}(\omega, r)=-F A_{0}^{\prime} \frac{(l+2)(l-1)}{i \omega r} \tilde{W}(\omega, r),
\end{aligned}
$$

with $\tilde{W}(\omega, r)$ being the Fourier transform of the time dependent function

$$
W(t, r)=\frac{F(r)}{r} h_{1}(t, r)
$$

Equations (5.64) are both in the Regge-Wheeler form, and they agree with both Chandrasekhar and Zerilli results; as it can be seen, in the limits $A_{0}(r) \rightarrow 0$ and $a_{B}(t, r) \rightarrow 0$ the Schwarzschild case is recovered. When the gauge vector is on, the vector perturbation works as a source for the metric's equation, and on the other hand the metric perturbations act as a source for the vector equation. However, it is important to notice that equations (5.64) contain only axial functions, and hence in this case there is no parity mixing, as it happens in the Schwarzschild case. 


\subsection{Magnetic Reissner-Nordström case}

In this case, the line element describing the background spacetime is

$$
d s^{2}=-F(r) d t^{2}+F(r)^{-1} d r^{2}+r^{2} d \theta^{2}+r^{2} \sin ^{2} \theta d \varphi^{2}
$$

with $F(r)=1-2 M / r+P^{2} / r^{2}$ and with background gauge vector

$$
A_{\mu}=\{0,0,0,-P \cos \theta\},
$$

being $P$ the magnetic charge. As in the electric case, there is no scalar field supported $(\phi=0, \delta \phi=0)$ and no contribution from the Chern-Simons term $\left(\phi \tilde{F}^{\mu \nu} F_{\mu \nu}=0\right)$. Since in the Schwarzschild and in the electric Reissner-Nordström case the $\{(t \varphi),(r \varphi),(\theta \varphi)\}$ components of the Einstein's Equations are separable in axial and polar equations due to the orthogonality properties of the spherical harmonics, let's study these components:

$$
\begin{aligned}
& (t \varphi):\left\{F\left[\frac{2}{r} \partial_{t} h_{1}+\partial_{t} \partial_{r} h_{1}-\partial_{r}^{2} h_{0}\right]+h_{0}\left[F^{\prime \prime}+\frac{2 F^{\prime}}{r}+\frac{2 F-2}{r^{2}}\right]+\frac{4 P}{r^{2}}\left[\partial_{t} a_{E}-a_{0}\right]\right. \\
& \left.+\frac{2 P^{2} h_{0}}{r^{4}}\right\} S_{\theta} Y(\theta, \varphi)-\frac{h_{0}}{r^{2}} S_{\theta}\left[\frac{1}{\sin ^{2} \theta} \partial_{\theta}^{2} Y(\theta, \varphi)+\frac{\cos \theta}{\sin \theta} \partial_{\theta} Y(\theta, \varphi)+\partial_{\varphi}^{2} Y(\theta, \varphi)\right] \\
& +\left[F^{\prime} H_{1}+F \partial_{r} H_{1}-\partial_{t} H_{0}-\partial_{t} K+\frac{4 P}{r^{2}} \partial_{t} a_{B}\right] \partial_{\varphi} Y(\theta, \varphi)=0 \\
& (r \varphi):\left\{\frac{1}{F}\left[\partial_{t}^{2} h_{1}-\partial_{r} \partial_{t} h_{0}+\frac{2}{r} \partial_{t} h_{0}\right]+h_{1}\left[F^{\prime \prime}+2 \partial_{r} \frac{F}{r}\right]+\frac{4 P}{r^{2}}\left[\partial_{r} a_{E}-a_{R}\right]\right. \\
& \left.+\frac{2 P^{2} h_{1}}{r^{4}}\right\} S_{\theta} Y(\theta, \varphi)-\frac{h_{1}}{r^{2}} S_{\theta}\left[\frac{1}{\sin ^{2} \theta} \partial_{\theta}^{2} Y(\theta, \varphi)+\frac{\cos \theta}{\sin \theta} \partial_{\theta} Y(\theta, \varphi)+\partial_{\varphi}^{2} Y(\theta, \varphi)\right] \\
& +\left[\frac{F^{\prime} H_{0}+F \partial_{r} H_{0}-F \partial_{r} K-\partial_{t} H_{1}}{F}+\frac{4 P}{r^{2}} \partial_{r} a_{B}\right] \partial_{\varphi} Y(\theta, \varphi)=0 \\
& (\theta \varphi): \frac{\partial_{t} h_{0}-F F^{\prime} h_{1}-F^{2} \partial_{r} h_{1}}{F}\left[\frac{1}{\sin ^{2} \theta} \partial_{\varphi}^{2} Y(\theta, \varphi)+\frac{\cos \theta}{\sin \theta} \partial_{\theta} Y(\theta, \varphi)+\partial_{\theta}^{2} Y(\theta, \varphi)\right] \sin \theta \\
& +\left[H_{0}-H_{2}\right]\left[\frac{1}{\sin ^{2} \theta} \partial_{\varphi} Y(\theta, \varphi)-\partial_{\varphi} \partial_{\theta} Y(\theta, \varphi)\right]=0
\end{aligned}
$$




\section{BLACK HOLE PERTURBATIONS}

where we have written $S_{\theta}=\sin \theta \partial_{\theta}$ for brevity.

Remarkably, since the angular part of the axial sector is no longer orthogonal to the polar one, we notice that the gauge component $A_{\varphi} \propto P$ mixes the axial and the polar sectors. Specifically, $A_{\varphi}$ couples the vector axial function $a_{B}(t, r)$ to the polar sector of the metric, and the polar functions $\left\{a_{0}(t, r), a_{R}(t, r), a_{E}(t, r)\right\}$ to the axial metric's sector.

From the previous three equations, introducing the tortoise radial derivative $\partial_{*}$ and the Fourier transform function

$$
\tilde{W}(\omega, r)=\int e^{i \omega t} W(t, r) d t
$$

of the time dependent function

$$
W(t, r)=\frac{F(r)}{r} h_{1}(t, r),
$$

with a bit of calculation we arrive at a single Regge-Wheeler equation:

$$
\left(\partial_{*}^{2}+\omega^{2}\right) \tilde{W}(\omega, r)-F(r)\left[\frac{l(l+1)}{r^{2}}-\frac{6 M}{r^{3}}+\frac{8 P^{2}}{r^{4}}\right] \tilde{W}(\omega, r)=s_{P}(\omega, r) .
$$

with source term

$$
s_{P}(\omega, r)=-4 P \frac{F(r)^{2}}{r^{3}}\left[\tilde{a}_{0}(\omega, r)+i \omega \tilde{a}_{E}(\omega, r)\right]
$$

Comparing (5.7]) with the analogous equation (5.641.a) for the electric case, we notice that although the structure of the two equations is identical (they are both written in a Regge-Wheeler form), the last equation mixes functions with different parity, while in the electric case only axial functions appeared. In particular, we notice that in the magnetic case the source term of the axial metric function is a polar term, specifically a function of two polar vector components $\left(a_{0}\right.$ and $\left.a_{E}\right)$. 
Moreover, from the component $A_{\theta}$ of the vector equation we get:

$$
\begin{aligned}
A_{\theta}: & \left\{r^{2}\left[F^{2} \partial_{r}^{2} a_{B}+F F^{\prime} \partial_{r} a_{B}-\partial_{t}^{2} a_{B}\right]+P F K\right\} \partial_{\varphi} Y(\theta, \varphi) \\
& +F a_{B} \partial_{\varphi}\left[\frac{1}{\sin ^{2} \theta} \partial_{\varphi}^{2} Y(\theta, \varphi)+\frac{\cos \theta}{\sin \theta} \partial_{\theta} Y(\theta, \varphi)+\partial_{\theta}^{2} Y(\theta, \varphi)\right] \\
& +r^{2}\left\{F F^{\prime}\left[\partial_{r} a_{E}-a_{R}\right]+F^{2}\left[\partial_{r}^{2} a_{E}-\partial_{r} a_{R}\right]+\partial_{t} a_{0}-\partial_{t}^{2} a_{E}\right. \\
& \left.+P\left[F^{2} \partial_{r} h_{1}-2 \frac{F^{2}}{r} h_{1}-\partial_{t} h_{0}+F F^{\prime} h_{1}\right]\right\} \sin \theta \partial_{\theta} Y(t, r)=0
\end{aligned}
$$

From the equation above, we notice that the gauge component $A_{\varphi} \propto P$ mixes the axial and the polar sectors, as it happens in the metric's equations, and therefore it is not possible to decouple the equation (5.7.3) into two separate axial and polar equations. Specifically, we notice that the $A_{\phi}$ component couples the polar function $K(t, r)$ to the axial equation (first 2 lines of equation (5.T3)), and the axial functions $h_{0}(t, r)$ and $h_{1}(t, r)$ to the polar equation (last 2 lines of (5.73)) . Considering the axial part of equation (5.73), after solving the angular part and introducing the tortoise derivative $\partial_{*}$ we arrive at

$$
\partial_{*}^{2} \tilde{a}_{B}(\omega, r)+\omega^{2} \tilde{a}_{B}(\omega, r)-F(r)\left[\frac{l(l+1)}{r^{2}}\right] \tilde{a}_{B}(\omega, r)=-P \frac{F(r)}{r^{2}} \tilde{K}(\omega, r),
$$

where for conveniency we moved into Fourier space.

The previous equation is written in a Regge-Wheeler form, but it is important to notice that the source term, namely the RHS of the equation, is a polar term, making the polarity mixing explicit.

We can try to make sense of these results if we look at the Einstein's Equation from a different prospective. At background level, we have

$$
R_{\mu \nu}=T_{\mu \nu}^{F} \propto \mathbf{B}^{2}
$$

where $\vec{B}$ is the magnetic field (axial vector) associated to the vector potential $\vec{A}$ such that $A_{\mu}=\{\Phi, \vec{A}\}$. Conceptually, perturbing at linear order the Einstein's equations means

$$
\delta g=\delta T
$$




\section{BLACK HOLE PERTURBATIONS}

with $g$ being the metric and with $\delta$ denoting the perturbation. Specifically, in the magnetic case we are considering we have

$$
\delta T \propto \delta g \mathbf{B}^{2}+g \delta B B
$$

and therefore we have

$$
\delta g \propto \delta g \mathbf{B}^{2}+g \delta B B
$$

Since the background metric and the magnetic field are even and odd quantities respectively, the previous equation formally reads

$$
\delta g=\delta g \cdot(E V E N)+\delta B \cdot(O D D)
$$

and therefore it is clear that in order to guarantee the consistency of the previous equation we need

$$
\delta g_{\text {even }}^{\text {odd }} \leftrightarrow \delta B_{\text {odd }}^{\text {even }}
$$

which agrees with the results we have found. So, due to the presence of an axial vector $(\vec{B})$ in the action, the metric's perturbations couple to vector's perturbations with opposite parity, although the action is globally parity invariant.

\subsection{Electric Chern-Simons case}

We now study the case of an electric Reissner-Nordström background including the perturbations of a scalar-vector Chern-Simons coupling of the form

$$
\phi \tilde{F}^{\mu \nu} F_{\mu \nu}
$$

This configuration is identical to the electric Reissner-Nordström one for both the background metric and the background gauge vector: 


$$
h_{\mu \nu}=\left(\begin{array}{cccc}
-F(r) & 0 & 0 & 0 \\
0 & F(r)^{-1} & 0 & 0 \\
0 & 0 & r^{2} & 0 \\
0 & 0 & 0 & r^{2} \sin ^{2} \theta
\end{array}\right)
$$

with

$$
F(r)=1-\frac{2 M}{r}+\frac{Q^{2}}{r^{2}}
$$

and where the gauge vector reads

$$
A_{\mu}=\left\{-\frac{Q}{r}, 0,0,0\right\}
$$

with $Q$ being the electric charge. However, since the Chern-Simons coupling is now on $\left(g_{F} \neq 0\right)$, we have to take into account the perturbations of the scalar field as well:

$$
\phi=0, \quad \delta \phi \neq 0
$$

Since the Chern-Simons does not contribute to the metric's equations of motion, they are formally identical to the electric Reissner-Nordström ones, even though the background vector field perturbations are directly coupled to the scalar field's ones. Hence, the metric's axial equation keeps the form

$$
\left(\partial_{*}^{2}+\omega^{2}\right) \tilde{W}(\omega, r)-F(r)\left[\frac{l(l+1)}{r^{2}}-\frac{6 M}{r^{3}}+\frac{4 Q^{2}}{r^{4}}\right] \tilde{W}(\omega, r)=-4 i \omega \frac{F(r) A_{0}^{\prime}(r)}{r} \tilde{a}_{B}(\omega, r) .
$$

Therefore, although not explicitly, the metric perturbations have to couple with the Chern-Simons term through the vector and scalar perturbations.

As a term of comparison, we remind that the electric Reissner-Nordström case was parity decoupled, with the axial perturbations being decoupled from the polar ones; on the contrary, the magnetic Reissner-Nordström case showed a mixing of terms with different parity. 


\section{BLACK HOLE PERTURBATIONS}

\section{Axial vector field's equation}

Instead, the vector equations get contributions from the Chern-Simons term, and they read:

$A_{t}: \operatorname{Fr}\left\{\left[K-H_{0}\right]\left[2 A_{0}^{\prime}+r A_{0}^{\prime \prime}\right]+r\left[A_{0}^{\prime} \partial_{r} K+\partial_{r}^{2} a_{0}-\partial_{t} \partial_{r} a_{R}\right]+2 \partial_{r} a_{0}-2 \partial_{t} a_{R}\right\} Y(\theta, \varphi)$

$+\left[a_{0}-\partial_{t} a_{E}\right]\left[\frac{1}{\sin ^{2} \theta} \partial_{\varphi}^{2} Y(\theta, \varphi)+\frac{\cos \theta}{\sin \theta} \partial_{\theta} Y(\theta, \varphi)+\partial_{\theta}^{2} Y(\theta, \varphi)\right]=0$,

$A_{r}:\left\{\frac{r^{2}}{F}\left[A_{0}^{\prime} \partial_{t} K+\partial_{t} \partial_{r} a_{0}-\partial_{t}^{2} a_{R}\right]-r H_{1}\left[2 A_{0}^{\prime}+r A_{0}^{\prime \prime}\right]\right\} Y(\theta, \varphi)$

$+\left[a_{R}-\partial_{r} a_{E}\right]\left[\frac{1}{\sin ^{2} \theta} \partial_{\varphi}^{2} Y(\theta, \varphi)+\frac{\cos \theta}{\sin \theta} \partial_{\theta} Y(\theta, \varphi)+\partial_{\theta}^{2} Y(\theta, \varphi)\right]=0$,

$A_{\theta}:\left\{\left[F^{2} \partial_{r}^{2}+F F^{\prime} \partial_{r}-\partial_{t}^{2}\right] a_{B}+F A_{0}^{\prime}\left[2 \frac{h_{0}}{r}+\partial_{t} h_{1}-\partial_{r} h_{0}\right]+2 g_{F} F \delta \phi A_{0}^{\prime}\right\} \partial_{\varphi} Y(\theta, \varphi)$

$+\frac{F}{r^{2}} a_{B} \partial_{\varphi}\left[\frac{1}{\sin ^{2} \theta} \partial_{\varphi}^{2} Y(\theta, \varphi)+\frac{\cos \theta}{\sin \theta} \partial_{\theta} Y(\theta, \varphi)+\partial_{\theta}^{2} Y(\theta, \varphi)\right]$

$+\left\{F F^{\prime}\left[\partial_{r} a_{E}-a_{R}\right]+F^{2}\left[\partial_{r}^{2} a_{E}-\partial_{r} a_{R}\right]+\partial_{t} a_{0}-\partial_{t}^{2} a_{E}\right\} \sin \theta \partial_{\theta} Y(t, r)=0$,

$A_{\varphi}:\left\{\left[\partial_{t}^{2}-F \partial_{r}\left(F \partial_{r}\right)\right] a_{B}-F A_{0}^{\prime}\left[\frac{2 h_{0}}{r}+\partial_{t} h_{1}-\partial_{r} h_{0}\right]-2 g_{F} F \delta \phi A_{0}^{\prime}\right\} \sin \theta \partial_{\theta} Y(\theta, \varphi)$

$+\frac{F}{r^{2}} a_{B} \sin \theta \partial_{\theta}\left[\frac{1}{\sin ^{2} \theta} \partial_{\theta}^{2} Y(\theta, \varphi)+\frac{\cos \theta}{\sin \theta} \partial_{\theta} Y(\theta, \varphi)+\partial_{\varphi}^{2} Y(\theta, \varphi)\right]$

$+\left\{F F^{\prime}\left[\partial_{r} a_{E}-a_{R}\right]+F^{2}\left[\partial_{r}^{2} a_{E}-\partial_{r} a_{R}\right]+\partial_{t} a_{0}-\partial_{t}^{2} a_{E}\right\} \partial_{\varphi} Y(\theta, \varphi)=0$.

We can notice that the equation for $A_{\theta}$ is just the corresponding electric ReissnerNordström equation with the addition of the $g_{F}$ term, which appears in the axial part of the equation since it has the same angular dependence of the axial contribution, which is orthogonal to the polar one. Hence, as we did in the ReissnerNordström case, we can write the equation of the axial vector component $a_{B}$ :

$$
\partial_{*}^{2} \tilde{a}_{B}(\omega, r)+\omega^{2} \tilde{a}_{B}(\omega, r)-F(r)\left[\frac{l(l+1)}{r^{2}}+\frac{4 Q^{2}}{r^{4}}\right] \tilde{a}_{B}(\omega, r)=\tilde{s}_{B}(\omega, r),
$$

with

$$
\tilde{s}_{B}(\omega, r)=-F(r) A_{0}^{\prime}(r) \frac{(l+2)(l-1)}{i \omega r} \tilde{W}(\omega, r)-2 g_{F} F(r) A_{0}^{\prime}(r) \tilde{\delta \phi}(\omega, r),
$$


where for convenience we used the Fourier transform functions $\tilde{a}_{B}$ and $\tilde{\delta \phi}$.

\section{Scalar field's equation}

The scalar field's equation can be written as

$$
\begin{aligned}
& \left\{r^{2} F \partial_{r}^{2} \delta \phi-\frac{r^{2}}{F} \partial_{t}^{2} \delta \phi+r\left[r F^{\prime}+2 F\right] \partial_{r} \delta \phi\right\} Y(\theta, \varphi) \\
& +\left[\delta \phi-g_{F} a_{B} A_{0}^{\prime}\right]\left[\frac{1}{\sin ^{2} \theta} \partial_{\varphi}^{2} Y(\theta, \varphi)+\frac{\cos \theta}{\sin \theta} \partial_{\theta} Y(\theta, \varphi)+\partial_{\theta}^{2} Y(\theta, \varphi)\right]=0 .
\end{aligned}
$$

Again, we perform a separation of variables and we first solve the angular equation, which is again the General Legendre Equation whose solution are the spherical harmonics $Y_{l m}(\theta, \varphi)$ and the separation constant is $\lambda=l(l+1)$. So, the radial equation we are left to solve is

$$
r^{2} F \partial_{r}^{2} \delta \phi-\frac{r^{2}}{F} \partial_{t}^{2} \delta \phi+r\left[r F^{\prime}+2 F\right] \partial_{r} \delta \phi-l(l+1)\left[\delta \phi-g_{F} a_{B} A_{0}^{\prime}\right]=0 .
$$

From the previous equation, after some manipulations we finally arrive at:

$$
\partial_{*}^{2} \delta \phi(t, r)-\partial_{t}^{2} \delta \phi(t, r)-\frac{F}{r^{2}}\left[l(l+1)+r F^{\prime}\right] \delta \phi(t, r)=-g_{F} l(l+1) \frac{F A_{0}^{\prime}}{r} a_{B}(t, r),
$$

where we have defined

$$
\delta \phi(t, r)=\frac{1}{r} \delta \phi(t, r)
$$

and the tortoise derivative

$$
\partial_{*}=F(r) \partial_{r}
$$

Even if it's supposed to be a polar function, equation (5.92) satisfies a ReggeWheleer like equation, with potential given by

$$
V(r)=\frac{F(r)}{r^{2}}\left[l(l+1)-r F^{\prime}(r)\right] .
$$




\section{BLACK HOLE PERTURBATIONS}

To recap, we the three equations describing the axial sector are:

$$
\begin{aligned}
& {\left[\partial_{*}^{2}+\omega^{2}\right] \tilde{W}(\omega, r)-F\left[\frac{l(l+1)}{r^{2}}-\frac{6 M}{r^{3}}+\frac{4 Q^{2}}{r^{4}}\right] \tilde{W}(\omega, r)=-4 i \omega \frac{F A_{0}^{\prime}}{r} \tilde{a}_{B}(\omega, r),} \\
& \partial_{*}^{2} \tilde{a}_{B}(\omega, r)+\omega^{2} \tilde{a}_{B}(\omega, r)-F\left[\frac{l(l+1)}{r^{2}}+\frac{4 Q^{2}}{r^{4}}\right] \tilde{a}_{B}(\omega, r)=\tilde{s}_{B}(\omega, r), \\
& \partial_{*}^{2} \tilde{\Phi}_{0}(\omega, r)+\omega^{2} \tilde{\Phi}_{0}(\omega, r)-\frac{F}{r^{2}}\left[l(l+1)+r F^{\prime}\right] \tilde{\Phi}_{0}(\omega, r)=-g_{F} l(l+1) \frac{F A_{0}^{\prime}}{r} \tilde{a}_{B}(\omega, r),
\end{aligned}
$$

with $\tilde{s}_{B}$ defined as

$$
\tilde{s}_{B}(\omega, r)=-F A_{0}^{\prime} \frac{(l+2)(l-1)}{i \omega r} \tilde{W}(\omega, r)-2 g_{F} \frac{F A_{0}^{\prime}}{r} \tilde{\delta \phi}(\omega, r) .
$$

A schematic diagram of the connections between metric-vector-scalar perturbations is given below in Figure 5.3 .

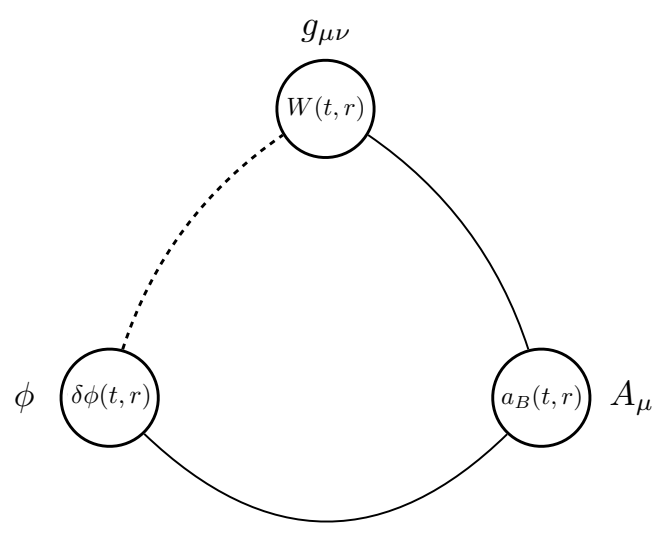

Figure 5.3: Schematic diagram of the axial sector for metric, vector and scalar perturbations. The axial metric's perturbations $W(t, r)$ are sourced by the vector ones $a_{B}(t, r)$, which are sourced by the scalar field perturbations $\delta \phi(t, r)$. Therefore, although the link is not explicitly manifested from the equations, the metric's perturbations implicitly depend on the scalar ones. In the picture, the solid black lines denotes the "direct" dependencies, while the dashed one denotes the "implicit" dependency metric/scalar. 


\subsection{Magnetic Chern-Simons case}

The last case we study is the one with a magnetic Reissner-Nordström background with an additional scalar-vector Chern-Simons coupling, similarly to what we did in the previous section for the electric case. Again, we consider a vanishing scalar field on the background, hence enhancing only the perturbations of the scalar field and consequently the Chern-Simons ones:

$$
\phi=0, \quad \delta \phi \neq 0
$$

Since the scalar field does not backreact with the geometry, the configuration we are studying is identical to the magnetic Reissner-Nordström one for both the background metric and the background gauge vector:

$$
h_{\mu \nu}=\left(\begin{array}{cccc}
-F(r) & 0 & 0 & 0 \\
0 & F(r)^{-1} & 0 & 0 \\
0 & 0 & r^{2} & 0 \\
0 & 0 & 0 & r^{2} \sin ^{2} \theta
\end{array}\right) \text {, }
$$

with

$$
F(r)=1-\frac{2 M}{r}+\frac{P^{2}}{r^{2}},
$$

and where the gauge vector reads

$$
A_{\mu}=\{0,0,0,-P \cos \theta\}
$$

with $P$ being the magnetic charge.

Given that in the magnetic Reissner-Nordström case we found a mixing of the parity sectors, we now investigate if that still holds when an extra parity violating term is taken into action. As in the previous case, since the Chern-Simons does not contribute to the metric's equations of motion they are formally identical to the electric Reissner-Nordström ones, and the axial metric's equation still reads $\left[\partial_{*}^{2}+\omega^{2}\right] \tilde{W}(\omega, r)-F\left[\frac{l(l+1)}{r^{2}}-\frac{6 M}{r^{3}}+\frac{8 P^{2}}{r^{4}}\right] \tilde{W}(\omega, r)=-\frac{4 P F^{2}}{r^{3}}\left[\tilde{a}_{0}(\omega, r)+i \omega \tilde{a}_{E}(\omega, r)\right]$. 


\section{BLACK HOLE PERTURBATIONS}

However, as it happens in the electric Chern-Simons case, since the metric perturbations are coupled to the vector ones and these are coupled to the scalar field's ones, the metric perturbations turn to be dependent on the scalar ones as well, even though not explicitely.

\section{Vector field's equation}

As it happens for the electric Chern-Simons case, the vector equations get contributions from the Chern-Simons term, and they read:

$$
\begin{aligned}
A_{t}: & +r F\left[2 \partial_{r} a_{0}+r \partial_{r}^{2} a_{0}-2 \partial_{t} a_{R}-r \partial_{t} \partial_{r} a_{R}+2 g_{F} P \frac{\partial_{r} \delta \phi}{r}\right] Y(\theta, \varphi) \\
& +\left[a_{0}-\partial_{t} a_{E}-P \frac{h_{0}}{r}\right]\left[\frac{1}{\sin ^{2} \theta} \partial_{\varphi}^{2} Y(\theta, \varphi)+\frac{\cos \theta}{\sin \theta} \partial_{\theta} Y(\theta, \varphi)+\partial_{\theta}^{2} Y(\theta, \varphi)\right]=0 \\
A_{r}: \quad & +r^{2}\left[\partial_{t} \partial_{r} a_{0}-\partial_{t}^{2} a_{R}+2 g_{F} P \frac{\partial_{t} \delta \phi}{r^{2}}\right] Y(\theta, \varphi) \\
& +F\left[a_{R}-\partial_{r} a_{E}-P \frac{h_{1}}{r^{2}}\right]\left[\frac{1}{\sin ^{2} \theta} \partial_{\varphi}^{2} Y(\theta, \varphi)+\frac{\cos \theta}{\sin \theta} \partial_{\theta} Y(\theta, \varphi)+\partial_{\theta}^{2} Y(\theta, \varphi)\right]=0 \\
A_{\theta}:\{ & \left.r^{2}\left[F^{2} \partial_{r}^{2} a_{B}+F F^{\prime} \partial_{r} a_{B}-\partial_{t}^{2} a_{B}\right]+P F K\right\} \partial_{\varphi} Y(\theta, \varphi) \\
& +F a_{B} \partial_{\varphi}\left[\frac{1}{\sin ^{2} \theta} \partial_{\varphi}^{2} Y(\theta, \varphi)+\frac{\cos \theta}{\sin \theta} \partial_{\theta} Y(\theta, \varphi)+\partial_{\theta}^{2} Y(\theta, \varphi)\right] \\
& +r^{2}\left\{F F^{\prime}\left[\partial_{r} a_{E}-a_{R}\right]+F^{2}\left[\partial_{r}^{2} a_{E}-\partial_{r} a_{R}\right]+\partial_{t} a_{0}-\partial_{t}^{2} a_{E}\right. \\
& \left.+P\left[F^{2} \partial_{r} h_{1}-2 \frac{F^{2}}{r} h_{1}-\partial_{t} h_{0}+F F^{\prime} h_{1}\right]\right\} \sin \theta \partial_{\theta} Y(t, r)=0 \\
A_{\varphi}:\{ & \left.r^{2}\left[\partial_{t}^{2} a_{B}-F^{2} \partial_{r}^{2} a_{B}-F F^{\prime} \partial_{r} a_{B}\right]-P F K\right\} \sin \theta \partial_{\theta} Y(\theta, \varphi) \\
& +F a_{B} \sin \theta \partial_{\theta}\left[\frac{1}{\sin ^{2} \theta} \partial_{\theta}^{2} Y(\theta, \varphi)+\frac{\cos \theta}{\sin \theta} \partial_{\theta} Y(\theta, \varphi)+\partial_{\theta}^{2} Y(\theta, \varphi)\right] \\
& +r^{2}\left\{F F^{\prime}\left[\partial_{r} a_{E}-a_{R}\right]+F^{2}\left[\partial_{r}^{2} a_{E}-\partial_{r} a_{R}\right]+\partial_{t} a_{0}-\partial_{t}^{2} a_{E}\right. \\
& \left.+P\left[F F^{\prime} h_{1}+F^{2} \partial_{r} h_{1}-2 h_{1} \frac{F^{2}}{r}-\partial_{t} h_{0}\right]\right\} \partial_{\varphi} Y(\theta, \varphi)=0
\end{aligned}
$$

We notice that the Chern-Simons terms (recognizable by the coupling $g_{F}$ ) do not enter in the equations of the axial vector component $a_{B}(t, r)$, which therefore still 
satisfies the equation:

$$
\partial_{*}^{2} \tilde{a}_{B}(\omega, r)+\omega^{2} \tilde{a}_{B}(\omega, r)-F(r)\left[\frac{l(l+1)}{r^{2}}\right] \tilde{a}_{B}(\omega, r)=-P \frac{F(r)}{r^{2}} \tilde{K}(\omega, r),
$$

as in the magnetic Reissner-Nordström case we discussed in the previous section. Apparently, the Chern-Simons coupling term does not interact with the axial gauge vector components; however, from the equations (5.103.1) and (5.10.3.2), we notice that the Chern-Simons coupling term interacts with the polar vector components $\left\{a_{0}(t, r), a_{R}(t, r), a_{E}(t, r)\right\}$. Therefore, taking cognizance of the axial metric equation (5.102), it is clear that the Chern-Simons contributions affect the axial metric component, coupling the scalar field's perturbations to the vector polar ones and therefore to the metric as well.

\section{Scalar field's equation}

The perturbations equation of the scalar field reads:

$$
\begin{aligned}
& \left\{r^{2} F \partial_{r}^{2} \delta \phi-\frac{r^{2}}{F} \partial_{t}^{2} \delta \phi+r\left[r F^{\prime}+2 F\right] \partial_{r} \delta \phi+g_{F} P\left[\partial_{r} a_{0}-\partial_{t} a_{E}\right]\right\} Y(\theta, \varphi) \\
& +\delta \phi\left[\frac{1}{\sin ^{2} \theta} \partial_{\varphi}^{2} Y(\theta, \varphi)+\frac{\cos \theta}{\sin \theta} \partial_{\theta} Y(\theta, \varphi)+\partial_{\theta}^{2} Y(\theta, \varphi)\right]=0
\end{aligned}
$$

where for simplicity we have already used the background solution

$$
A_{\varphi}=-P \cos \theta
$$

As in the previous case, we can perform a separation of variables and solve the angular equation

$$
\left[\frac{1}{\sin ^{2} \theta} \partial_{\varphi}^{2} Y(\theta, \varphi)+\frac{\cos \theta}{\sin \theta} \partial_{\theta} Y(\theta, \varphi)+\partial_{\theta}^{2} Y(\theta, \varphi)\right]+\lambda Y(\theta, \varphi)=0,
$$

with separation constant $\lambda$ given as usual by

$$
\lambda=l(l+1) .
$$




\section{BLACK HOLE PERTURBATIONS}

After redefining the scalar field as

$$
\delta \phi(t, r)=\frac{1}{r} \delta \phi(t, r)
$$

and introducing the tortoise derivative $\partial_{*}=F(r) \partial_{r}$, we finally arrive at the following equation for the scalar field's perturbations:

$$
\partial_{*}^{2} \delta \phi(t, r)-\partial_{t}^{2} \delta \phi(t, r)-\frac{F}{r^{2}}\left[l(l+1)+r F^{\prime}\right] \delta \phi(t, r)=g_{F} P \frac{F \partial_{t} a_{E}(t, r)-\partial_{*} a_{0}(t, r)}{r} .
$$

To recap, the equations we found are:

$$
\begin{aligned}
& {\left[\partial_{*}^{2}+\omega^{2}\right] \tilde{W}(\omega, r)-F\left[\frac{l(l+1)}{r^{2}}-\frac{6 M}{r^{3}}+\frac{8 P^{2}}{r^{4}}\right] \tilde{W}(\omega, r)=-\frac{4 P F^{2}}{r^{3}}\left[\tilde{a}_{0}(\omega, r)+i \omega \tilde{a}_{E}(\omega, r)\right]} \\
& \partial_{*}^{2} \tilde{a}_{B}(\omega, r)+\omega^{2} \tilde{a}_{B}(\omega, r)-F\left[\frac{l(l+1)}{r^{2}}\right] \tilde{a}_{B}(\omega, r)=-P \frac{F}{r^{2}} \tilde{K}(\omega, r) \\
& \partial_{*}^{2} \delta \phi(t, r)-\partial_{t}^{2} \delta \phi(t, r)-\frac{F}{r^{2}}\left[l(l+1)+r F^{\prime}\right] \delta \phi(t, r)=g_{F} P \frac{F \partial_{t} a_{E}(t, r)-\partial_{*} a_{0}(t, r)}{r} .
\end{aligned}
$$

A schematic diagram of the connections between metric-vector-scalar perturbations is given below in Figure 5.4.

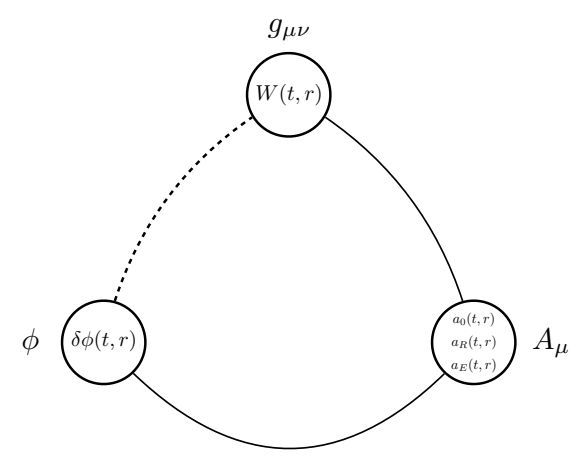

Figure 5.4: Schematic diagram of the mixed metric, vector and scalar perturbations. The axial metric's perturbations $W(t, r)$ are sourced by the polar vector ones $a_{0}(t, r), a_{R}(t, r), a_{E}(t, r)$, which are sourced by the scalar field perturbations $\delta \phi(t, r)$. Although not explicitly, the metric's perturbations implicitly depend on the scalar ones through the polar vector perturbations. The solid black lines denotes the "direct" dependencies, while the dashed one denotes the "implicit" one. 


\subsection{Conclusions}

In this chapter we have studied the perturbations of Reissner-Nordström spacetime in the presence of magnetic charge (5.5) and Chern-Simons scalar-vector couplings $(5.6,5.7)$. In these cases, we have found a mixing of perturbations (gravitational, electromagnetic and scalar) with opposite parity. Since the parity decoupling usually leads to two independent Schrödinger equations with the same QNMs spectrum, a parity mixing behaviour can be a distinguishable, distinctive feature of such black hole systems. Moreover, an accurate QNMs analysis could eventually reveal any detectable effect through Gravitational Waves emission, giving us an ulterior tool to test alternative theories of gravity. Therefore, although an accurate QNMs analysis is beyond the aim of this project, it should be still considered a very interesting area to be further investigated. 


\section{Chapter 6}

\section{Conclusions}

In this thesis we studied various aspects of modern cosmology related with black hole physics. In particular, encouraged by the great impact the GW150914 detection and the subsequents ones have had, we investigated black holes physical phenomena which could provide detectable effects in the context of testing General Relativity or alternative theories of gravity, with particular interest to the case of theories involving extra scalar (axion) fields. Before discussing possible further developments and future investigations, we now briefly summarize the main results of this work.

In chapter [2, we studied an exact, rotating black hole solution arising from a vector-tensor theory, obtained through a disformal transformation of the EinsteinMaxwell solution. In particular, we used as disformal vector the gauge vector satisfying the Einstein-Maxwell equations of motion. We found that such disformal transformation caused the gauge invariance to be explicitly broken, losing the gauge freedom to arbitrarily remove some components of the gauge vector. In-

stead, depending on the particular choice of the radial and polar components of the vector, the resulting geometry could be either a black hole or a naked singularity. Thus, we determined a particular choice of the gauge vector which makes the geometry a static/stationary black hole everywhere regular outside an event horizon, with both the horizon and the ergosurface depending on the disformal coupling. For such solution, we studied the ISCOs and the maximum amount of 
extractable binding energy.

In chapter 3 , we considered the interaction between a rotating, charged black hole and an external, ultralight scalar field. In particular, we studied the scalar cloud formation around an extremal electromagnetic Kerr-Newman black hole, extending previous results obtained for an electric black hole to an electromagnetic one. We found that depending on the frequency of the scalar modes the system could trigger superradiance, leading to the formation of a long-lived (stationary) scalar cloud surrounding the black hole. Interestingly, the frequencies of the modes of the scalar cloud correspond to the resonant frequencies of the superradiant gain factor, which is totally determined by the black hole's and scalar's parameters.

In chapter $⿴$, we studied electromagnetic black holes non minimally coupled with a massless scalar field through parity violating scalar-vector and scalar-tensor Chern-Simons coupling terms, namely $g_{F} \phi F_{\mu \nu} F^{\mu \nu}$ and $g_{R} \phi R^{\mu \nu \alpha \beta} R_{\mu \nu \alpha \beta}$, with $\phi$ being the scalar field and $g_{F}$ and $g_{R}$ being the coupling constants. We found that in the static, electromagnetic case there is a contribution from the scalarvector coupling to the geometry, with a spontaneous formation of a secondary scalar hair depending on the coupling $g_{F}$. In the case of rotating black holes, using a small couplings and a slow rotation approximation scheme we analytically found the hairy black hole solution depending on both $g_{F}$ and $g_{R}$. For such solutions, we verified that the thermodynamics Smarr formula is satisfied. Looking for detectable, distinctive phenomena, we calculated the ISCO trajectories and frequencies; moreover, we studied how the light traveling through the scalar field surrounding the black hole is subjected to a natural polarization splitting, arising from the parity violating scalar-vector coupling.

At the end, in chapter $\square$ we investigated the linear perturbation theory of an electromagnetic Reissner-Nordström black hole, also including a Chern-Simons scalar-vector coupling. Working in the Regge-Wheleer gauge, expanding the tensor, vector and scalar perturbations into axial/polar components we found that in the Reissner-Nordström magnetic case the resulting Regge-Wheeler equation for 
parity odd perturbations contains both polar and axial terms, unlikely what happens in the electric case. Moreover, such parity mixing also appears when we take into account the Chern-Simons scalar-vector coupling, leading to a Regge-Wheeler gravitational perturbations equation involving both vector and scalar perturbations with different parity.

The main idea behind this work was to study various physical systems involving black hole physics, in order to find detectable, distinctive effects which could provide a tool to test and eventually falsify gravity theories. In doing this, we were motivated by the fact that black holes are still the best candidates for gravitational waves observable emissions, with current (LIGO, VIRGO, etc.) and next generations detectors (LISA, KAGRA, etc.). Thus, in this work we have provided some examples of non standard General Relativity black hole configurations with their distinctive, (maybe) observable traits. In particular, we focused our attention on systems with scalar fields, since they continue to be good candidates for solving relevant open questions, such as dark energy and dark matter. Therefore, we think that further investigations are necessary to explore new scenarios, supported by the observations from the current detectors and the ones which are to come. For interesting further developments of the results discussed in this work, we propose the following ideas.

- Since the black hole solution we found in chapter $\nabla$ comes from a very simple and ad hoc disformal transformation, it would be useful to investigate if a more general approach is viable. In particular, we think that a rigorous study of generic disformal transformations applied to known black hole solutions could be a fascinating systematic way to find exact black hole solutions in modified gravity theories, which is still an open and appealing question. About this topic, we mention [226, 227] for recent developments.

- On the basis of the hairy solution found in chapter $\mathbb{6}$, it would be interesting to try to extend such results to the case of arbitrarily fast rotating black holes, with also arbitrarily large couplings with Chern-Simons terms. 
Furthermore, a perturbations and QNMs analysis could reveal some distinctive feature of the Chern-Simons terms in the gravitational waves spectrum, making it comparable with the observations.

- In chapter 日e studied the linear perturbations for various black hole solutions, finding a peculiar parity mixing in some of them. For such cases, it would be interesting to undertake a full study of QNMs, looking for possible detectable modes comparable with waveforms coming from ringdown phases of binary systems. Moreover, it would be interesting to extend the analysis we made to the case of rotating black holes (electromagnetic Kerr-Newman), also including a Chern-Simons scalar-gravity coupling term. 


\section{References}

[1] C. Heinicke and F. W. Hehl, "Schwarzschild and Kerr Solutions of Einstein's Field Equation - an introduction," Int. J. Mod. Phys. D24 no. 02, (2014) 1530006, arXiv: 1503.02172 [gr-qc]. 四, 回, ㅁ.

[2] E. Berti et al., "Testing General Relativity with Present and Future Astrophysical Observations," Class. Quant. Grav. 32 (2015) 243001,

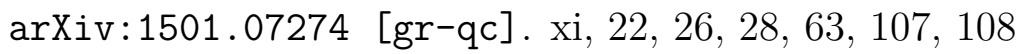

[3] LIGO Scientific, Virgo Collaboration, B. P. Abbott et al., "Observation of Gravitational Waves from a Binary Black Hole Merger," Phys. Rev. Lett. 116 no. 6, (2016) 061102, arXiv:1602.03837 Lgr-qc]. 区ய, उ4, 미, 마, 182

[4] A. Einstein, "Die Feldgleichungen der Gravitation," Sitzungsberichte der Königlich Preußischen Akademie der Wissenschaften (Berlin (Jan., 1915) 844-847. Ш, 田

[5] LIGO Scientific, Virgo Collaboration, B. P. Abbott et al., "Properties of the Binary Black Hole Merger GW150914," Phys. Rev. Lett. 116 no. 24, (2016) 241102, arXiv:1602.03840 [gr-qc]. П, В4, एण7

[6] L. Ackerman, M. R. Buckley, S. M. Carroll, and M. Kamionkowski, "Dark Matter and Dark Radiation," Phys. Rev. D79 (2009) 023519, arXiv:0810.5126 [hep-ph]. [,277(2008)]. [, प40

[7] N. Arkani-Hamed, D. P. Finkbeiner, T. R. Slatyer, and N. Weiner, "A Theory of Dark Matter," Phys. Rev. D79 (2009) 015014, arXiv:0810.0713 [hep-ph]. आ, 24, ㅁ.3, ए84 


\section{REFERENCES}

[8] T. Clifton, P. G. Ferreira, A. Padilla, and C. Skordis, "Modified Gravity and Cosmology," Phys. Rept. 513 (2012) 1-189, arXiv:1106.2476 [astro-ph.C0]. [2, [22, [25, 62, 미, ए3.

[9] S. Nojiri, S. Odintsov, and V. Oikonomou, "Modified gravity theories on a nutshell: Inflation, bounce and late-time evolution," Physics Reports 692 (Jun, 2017) 1-104. http://dx.doi.org/10.1016/j.physrep.2017.06.001.

[10] L. Heisenberg, "Generalization of the Proca Action," JCAP 1405 (2014) 015, arXiv:1402.7026 Lhep-th]. B], 62, 64

[11] A. De Felice, L. Heisenberg, R. Kase, S. Mukohyama, S. Tsujikawa, and Y.-l. Zhang, "Cosmology in generalized Proca theories," JCAP 1606 no. 06, (2016) 048, arXiv: 1603.05806 [gr-qc]. B], 67

[12] J. D. Bekenstein, "The Relation between physical and gravitational geometry," Phys. Rev. D48 (1993) 3641-3647, arXiv:gr-qc/9211017 [gr-qc]. [3], [6. [6], 67]

[13] D. Bettoni and S. Liberati, "Disformal invariance of second order scalar-tensor theories: Framing the Horndeski action," Phys. Rev. D88 (2013) 084020, arXiv:1306.6724 Lgr-qc]. [3, 63], 6.5, 66]

[14] J. E. Kim and G. Carosi, "Axions and the Strong CP Problem," Rev. Mod. Phys. 82 (2010) 557-602, arXiv:0807.3125 Lhep-ph]. [4, [36, ए0.9, ए32, ए33

[15] R. Jackiw and S. Y. Pi, "Chern-Simons modification of general relativity," Phys. Rev. D68 (2003) 104012, arXiv:gr-qc/0308071 Lgr-qc]. [1, [3.3, [3.9]

[16] S. Alexander and N. Yunes, "Chern-Simons Modified General Relativity," Phys. Rept. 480 (2009) 1-55, arXiv:0907.2562 [hep-th]. औ, [3], ⒊1, ए52

[17] L. Smarr, "Mass formula for Kerr black holes," Phys. Rev. Lett. 30 (1973) 71-73. [Erratum: Phys. Rev. Lett.30,521(1973)]. 由, ए2, प44 
[18] T. Regge and J. A. Wheeler, "Stability of a schwarzschild singularity," Phys. Rev. 108 (Nov, 1957) 1063-1069. https://link.aps.org/doi/10.1103/PhysRev.108.1063. ब, ए79, ए97

[19] M. Visser, "The Kerr spacetime: A Brief introduction," in Kerr Fest: Black Holes in Astrophysics, General Relativity and Quantum Gravity Christchurch, New Zealand, August 26-28, 2004. 2007. arXiv:0706.0622

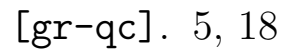

[20] S. A. Teukolsky, "The Kerr Metric," Class. Quant. Grav. 32 no. 12, (2015) [240)(16, arXiv: 1410.2130 [gr-qc]. [8, [4], [8]

[21] T. Adamo and E. T. Newman, "The Kerr-Newman metric: A Review," Scholarpedia 9 (2014) 31791, arXiv:1410.6626 Lgr-qc]. [1, ㅁ6, [201, [14]

[22] S. M. Carroll, Spacetime and geometry: An introduction to general relativity. 2004. http:

//wWw.slac.stanford.edu/spires/find/books/wWw?cl=QC6:C37:2004.

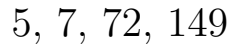

[23] J. B. Hartle, "Gravity: An Introduction to Einstein's General Relativity," American Journal of Physics 71 no. 10, (2003) 1086-1087,

https://doi.org/10.1119/1.1604390.

https://doi.org/10.1119/1.1604390. 82, 831, प92, प33, ㅁ61, ㅁ04, 마8

[24] T. Cheng, Relativity, Gravitation and Cosmology: A Basic Introduction.

Oxford Master Series in Physics. OUP Oxford, 2010.

https://books.google.co.uk/books?id=Q6p0DgAAQBAJ. [0]

[25] E. Poisson, A Relativist's Toolkit: The Mathematics of Black-Hole

Mechanics. Cambridge University Press, 2004. 띠, [12

[26] R. M. Wald, General relativity. Chicago Univ. Press, Chicago, IL, 1984. https://cds.cern.ch/record/106274. 8, ㅁ. 
[27] S. W. Hawking and G. F. R. Ellis, The Large Scale Structure of Space-Time. Cambridge Monographs on Mathematical Physics. Cambridge University Press, 1973. [, ए0]

[28] A. Einstein, "The Foundation of the General Theory of Relativity," Annalen Phys. 49 no. 7, (1916) 769-822. [Annalen Phys.354,no.7,769(1916)].

[29] K. Schwarzschild, "On the gravitational field of a mass point according to Einstein's theory," Sitzungsber. Preuss. Akad. Wiss. Berlin (Math. Phys.) 1916 (1916) 189-196, arXiv:physics/9905030 [physics]. [

[30] R. Penrose, "Gravitational collapse: The role of general relativity," Riv. Nuovo Cim. 1 (1969) 252-276. [Gen. Rel. Grav.34,1141(2002)]. ㅁ], प6, ए32

[31] K. A. e. a. The Event Horizon Telescope Collaboration, "First M87 Event Horizon Telescope Results. I. The Shadow of the Supermassive Black Hole," Astrophys. J. Lett. 875 (2019). https://doi.org/10.3847/2041-8213/ab0ec7. ㅁ., 34, ㅁ.3

[32] K. A. e. a. The Event Horizon Telescope Collaboration, "First M87 Event Horizon Telescope Results. II. Array and Instrumentation," Astrophys. J. Lett. 875 (2019). https://doi.org/10.3847/2041-8213/ab0c96.

[33] K. A. e. a. The Event Horizon Telescope Collaboration, "First M87 Event Horizon Telescope Results. III. Data Processing and Calibration," Astrophys. J. Lett. 875 (2019). https://doi.org/10.3847/2041-8213/ab0c57.

[34] K. A. e. a. The Event Horizon Telescope Collaboration, "First M87 Event Horizon Telescope Results. IV. Imaging the Central Supermassive Black Hole," Astrophys. J. Lett. 875 (2019). https://doi.org/10.3847/2041-8213/ab0e85.

[35] K. A. e. a. The Event Horizon Telescope Collaboration, "First M87 Event Horizon Telescope Results. V. Physical Origin of the Asymmetric Ring," 
Astrophys. J. Lett. 875 (2019) .

https://doi.org/10.3847/2041-8213/ab0f43.

[36] K. A. e. a. The Event Horizon Telescope Collaboration, "First M87 Event Horizon Telescope Results. VI. The Shadow and Mass of the Central Black Hole," Astrophys. J. Lett. 875 (2019). https://doi.org/10.3847/2041-8213/ab0f43. ㅁ., 34, ㅁ.3

[37] LIGO Scientific, Virgo Collaboration, B. P. Abbott et al., "GWTC-1: A Gravitational-Wave Transient Catalog of Compact Binary Mergers Observed by LIGO and Virgo during the First and Second Observing Runs," arXiv:1811.12907 [astro-ph.HE]. 미, [4, 62, ए08

[38] H. Reissner, "Über die eigengravitation des elektrischen feldes nach der einsteinschen theorie," Annalen der Physik 355 no. 9, (1916) 106-120, https://onlinelibrary.wiley.com/doi/pdf/10.1002/andp.19163550905. https:

//onlinelibrary.wiley.com/doi/abs/10.1002/andp.19163550905. [4]

[39] G. Nordström, "On the Energy of the Gravitation field in Einstein's Theory," Koninklijke Nederlandse Akademie van Wetenschappen Proceedings Series B Physical Sciences 20 (Jan, 1918) 1238-1245. ㄸ4

[40] R. P. Kerr, "Gravitational Field of a Spinning Mass as an Example of Algebraically Special Metrics," Phys. Rev. Lett. 11 (Sep, 1963) 237-238. https://link.aps.org/doi/10.1103/PhysRevLett.11.237. ㅁ6, ए79

[41] R. Penrose and R. M. Floyd, "Extraction of Rotational Energy from a Black Hole," Nature Physical Science 229 no. 6, (1971) 177-179. https://doi.org/10.1038/physci229177a0. ㅁ, 201, 201, प6], 미

[42] C. Rovelli, "Black hole entropy from loop quantum gravity," Phys. Rev. Lett. 77 (1996) 3288-3291, arXiv:gr-qc/9603063 [gr-qc]. ए0]

[43] R. Bousso, "The Holographic principle," Rev. Mod. Phys. 74 (2002) 82.5-874, arXiv:hep-th/0203101 [hep-th]. 


\section{REFERENCES}

[44] S. Carlip, "Black Hole Thermodynamics," Int. J. Mod. Phys. D23 (2014) [4:30102:3, arXiv:1410.1486 [gr-qc]. 201

[45] J. M. Bardeen, B. Carter, and S. W. Hawking, "The four laws of black hole mechanics," Comm. Math. Phys. 31 no. 2, (1973) 161-170. https://projecteuclid.org:443/euclid.cmp/1103858973. [D]

[46] R. M. Wald, "The thermodynamics of black holes," Living Rev. Rel. 4 (2001) 6, arXiv:gr-qc/9912119 [gr-qc]. ए0]

[47] K. S. Virbhadra, "Energy associated with a Kerr-Newman black hole," Phys. Rev. D 41 (Feb, 1990) 1086-1090. https://link.aps.org/doi/10.1103/PhysRevD.41.1086. 201]

[48] D. Christodoulou and R. Ruffini, "Reversible Transformations of a Charged Black Hole," Phys. Rev. D 4 (Dec, 1971) 3552-3555. https://link.aps.org/doi/10.1103/PhysRevD .4.3552. [20]

[49] J. D. Bekenstein, "Black Holes and Entropy," Phys. Rev. D 7 (Apr, 1973) 2333-2346. https://link.aps.org/doi/10.1103/PhysRevD.7.2333. 2]

[50] S. W. Hawking, "Gravitational Radiation from Colliding Black Holes," Phys. Rev. Lett. 26 (May, 1971) 1344-1346. https://link.aps.org/doi/10.1103/PhysRevLett.26.1344. एा]

[51] L. Barack et al., "Black holes, gravitational waves and fundamental physics: a roadmap," arXiv:1806.05195 [gr-qc]. [22, 107

[52] J. Frieman, M. Turner, and D. Huterer, "Dark Energy and the Accelerating Universe," Ann. Rev. Astron. Astrophys. 46 (2008) 385-432, arXiv:0803.0982 [astro-ph]. [2:3]

[53] M. P. Hobson, G. P. Efstathiou, and A. N. Lasenby, General Relativity: An Introduction for Physicists. Cambridge University Press, 2006. 24

[54] A. Vainshtein, "To the problem of nonvanishing gravitation mass," Physics Letters B 39 no. 3, (1972) 393 - 394. http: 
//wWw.sciencedirect.com/science/article/pii/0370269372901475.

\section{[26], 62}

[55] "An introduction to the Vainshtein mechanism," Class. Quant. Grav. 30 (2013) 184001, arXiv:1304.7240 [gr-qc]. ए26, ए44

[56] J. Khoury and A. Weltman, "Chameleon fields: Awaiting surprises for tests of gravity in space," Phys. Rev. Lett. 93 (2004) 171104, arXiv:astro-ph/0309300 [astro-ph]. [26], [1], 62]

[57] J. Khoury and A. Weltman, "Chameleon cosmology," Phys. Rev. D 69 (2004) 044026, arXiv:astro-ph/0309411. ए6, उ

[58] M. Milgrom, "A modification of the Newtonian dynamics as a possible alternative to the hidden mass hypothesis.," apj 270 (July, 1983) 365-370. 26]

[59] M. Milgrom, "A modification of the Newtonian dynamics - Implications for galaxies.," apj 270 (July, 1983) 371-389.

[60] M. Milgrom, "A modification of the newtonian dynamics : implications for galaxy systems.," apj 270 (July, 1983) 384-389.

[61] B. Famaey and S. S. McGaugh, "Modified newtonian dynamics (mond): Observational phenomenology and relativistic extensions," Living reviews in relativity 15 no. 1, (2012) 10-10.

https://pubmed.ncbi.nlm.nih.gov/28163623.

[62] M. Milgrom, "MOND theory," Can. J. Phys. 93 no. 2, (2015) 107-118, arXiv:1404.7661 Lastro-ph.CO]. [26]

[63] D. Lovelock, "The einstein tensor and its generalizations," Journal of Mathematical Physics 12 no. 3, (1971) 498-501,

https://doi.org/10.1063/1.1665613.

https://doi.org/10.1063/1.1665613. 26] 


\section{REFERENCES}

[64] D. Lovelock, "The fourdimensionality of space and the einstein tensor," Journal of Mathematical Physics 13 no. 6, (1972) 874-876, https://doi.org/10.1063/1.1666069. https://doi.org/10.1063/1.1666069. 26]

[65] R. P. Woodard, "Ostrogradsky's theorem on Hamiltonian instability," Scholarpedia 10 no. 8, (2015) 32243, arXiv:1506.02210 [hep-th]. ए7]

[66] T. Kaluza, "Zum Unitätsproblem der Physik," Sitzungsberichte der Königlich Preußischen Akademie der Wissenschaften (Berlin (Jan., 1921) 966-972. ए7, एप]

[67] O. Klein, "Quantentheorie und fünfdimensionale Relativitätstheorie," Zeitschrift fur Physik 37 no. 12, (Dec., 1926) 895-906. 7, ए39

[68] V. Faraoni, Cosmology in scalar tensor gravity, vol. 139. 2004. [28, 5.3]

[69] A. Salvio, "Quadratic Gravity," Front. in Phys. 6 (2018) 77, arXiv:1804.09944 [hep-th]. [28]

[70] V. Faraoni and E. Gunzig, "Einstein frame or Jordan frame?," Int. I. Theor. Phys. 38 (1999) 217-225, arXiv:astro-ph/9910176. उ0]

[71] M. Postma and M. Volponi, "Equivalence of the Einstein and Jordan frames," Phys. Rev. D 90 no. 10, (2014) 103516, arXiv:1407.6874 [astro-ph.CO]. [30

[72] D. M. Eardley, "Observable effects of a scalar gravitational field in a binary pulsar.," apjl 196 (Mar., 1975) L59-L62. В]

[73] T. Damour and G. Esposito-Farèse, "Nonperturbative strong-field effects in tensor-scalar theories of gravitation," Phys. Rev. Lett. 70 (Apr, 1993) 2220-2223. https://link.aps.org/doi/10.1103/PhysRevLett.70.2220. 31]

[74] G. W. Horndeski, "Second-order scalar-tensor field equations in a four-dimensional space," International Journal of Theoretical Physics 10 no. 6, (1974) 363-384. https://doi.org/10.1007/BF01807638. 3] 
[75] T. Kobayashi, M. Yamaguchi, and J. Yokoyama, "Generalized G-inflation: Inflation with the most general second-order field equations," Prog. Theor. Phys. 126 (2011) 511-529, arXiv:1105.5723 [hep-th]. एس

[76] C. Brans and R. H. Dicke, "Mach's principle and a relativistic theory of gravitation," Phys. Rev. 124 (Nov, 1961) 925-935. https://link.aps.org/doi/10.1103/PhysRev.124.925. B]

[77] D. Langlois and K. Noui, "Degenerate higher derivative theories beyond Horndeski: evading the Ostrogradski instability," JCAP 02 (2016) 034, arXiv:1510.06930 [gr-qc]. 四

[78] K. S. Stelle, "Renormalization of higher-derivative quantum gravity," Phys. Rev. D 16 (Aug, 1977) 953-969. https://link.aps.org/doi/10.1103/PhysRevD.16.953. B2

[79] J. H. Schwarz, "Dilaton - axion symmetry," in International Workshop on String Theory, Quantum Gravity and the Unification of Fundamental Interactions, pp. 503-520. 9, 1992. arXiv:hep-th/9209125. उ2

[80] P. Brax, C. van de Bruck, A.-C. Davis, and D. Shaw, "The Dilaton and Modified Gravity," Phys. Rev. D 82 (2010) 063519, arXiv:1005.3735 [astro-ph.CO]. B2]

[81] P. Kanti, N. E. Mavromatos, J. Rizos, K. Tamvakis, and E. Winstanley, "Dilatonic black holes in higher curvature string gravity," Phys. Rev. D54 (1996) 5049-5058, arXiv:hep-th/9511071 Lhep-th]. [33, [3.

[82] C. Burgess, "Introduction to Effective Field Theory," Ann. Rev. Nucl. Part. Sci. 57 (2007) 329-362, arXiv:hep-th/0701053. B3]

[83] N. Yunes and F. Pretorius, "Dynamical Chern-Simons Modified Gravity. I. Spinning Black Holes in the Slow-Rotation Approximation," Phys. Rev. D79 (2009) 084043, arXiv:0902.4669 [gr-qc]. [3.3, [.3प, ए.52 
[84] LIGO Scientific, Virgo Collaboration, B. Abbott et al., "Tests of general relativity with GW150914," Phys. Rev. Lett. 116 no. 22, (2016) 221101, arXiv:1602.03841 [gr-qc]. [Erratum: Phys.Rev.Lett. 121, 129902 (2018)]. उ5, एण7, एण8

[85] LIGO Scientific, Virgo Collaboration, B. Abbott et al., "Tests of General Relativity with GW170817," Phys. Rev. Lett. 123 no. 1, (2019) 0I1112, arXiv:1811.00364 Lgr-qc]. B5

[86] LIGO Scientific, Virgo Collaboration, B. P. Abbott et al., "GW170817: Observation of Gravitational Waves from a Binary Neutron Star Inspiral," Phys. Rev. Lett. 119 no. 16, (2017) 161101, arXiv:1710.05832 [gr-qc]. उ.5, ए18

[87] D. Langlois, R. Saito, D. Yamauchi, and K. Noui, "Scalar-tensor theories and modified gravity in the wake of GW170817," Phys. Rev. D97 no. 6, (2018) 061501, arXiv:1711.07403 [gr-qc]. 35, ए0.9

[88] J. M. Ezquiaga and M. Zumalacárregui, "Dark Energy After GW170817: Dead Ends and the Road Ahead," Phys. Rev. Lett. 119 no. 25, (2017) 251304, arXiv:1710.05901 Lastro-ph.CO].

[89] P. Creminelli and F. Vernizzi, "Dark Energy after GW170817 and GRB170817A," Phys. Rev. Lett. 119 no. 25, (2017) 251302, arXiv:1710.05877 [astro-ph.C0].

[90] O. J. Tattersall, P. G. Ferreira, and M. Lagos, "Speed of gravitational waves and black hole hair," Phys. Rev. D 97 no. 8, (2018) 084005, arXiv:1802.08606 [gr-qc]. B.

[91] D. J. E. Marsh, "Axion Cosmology," Phys. Rept. 643 (2016) 1-79, arXiv:1510.07633 Lastro-ph.C0]. 361, 38, 401, 0.9, ए32

[92] F. Wilczek, "Problem of Strong $P$ and $T$ Invariance in the Presence of Instantons," Phys. Rev. Lett. 40 (1978) 279-282. ए6, ㅍ.32 
[93] R. D. Peccei and H. R. Quinn, "CP Conservation in the Presence of Pseudoparticles," Phys. Rev. Lett. 38 (1977) 1440-1443. [,328(1977)]. \$6, उ7

[94] R. D. Peccei and H. R. Quinn, "Constraints Imposed by CP Conservation in the Presence of Instantons," Phys. Rev. D16 (1977) 1791-1797. उ6], ए3.

[95] S. Coleman, Aspects of Symmetry: Selected Erice Lectures. Cambridge University Press, 1988.

http://books.google.co.uk/books?id=PX2Al8LE9FkC. [36, 37

[96] P. Svrcek and E. Witten, "Axions In String Theory," JHEP 06 (2006) 051, arXiv:hep-th/0605206 [hep-th]. [36, [39, ㅁ.9, ए32, ए33.

[97] A. Arvanitaki, S. Dimopoulos, S. Dubovsky, N. Kaloper, and

J. March-Russell, "String Axiverse," Phys. Rev. D81 (2010) 123530,

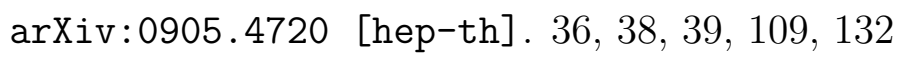

[98] R. D. Peccei, "The Strong CP problem and axions," Lect. Notes Phys. 741 (2008) 3-17, arXiv:hep-ph/0607268 Lhep-ph]. [,3(2006)]. 361, B8]

[99] R. Crewther, P. D. Vecchia, G. Veneziano, and E. Witten, "Chiral estimate of the electric dipole moment of the neutron in quantum chromodynamics," Physics Letters B 88 no. 1, (1979) 123 - 127. http:

//www.sciencedirect.com/science/article/pii/037026937990128X. 30

[100] C. A. Baker et al., "An Improved experimental limit on the electric dipole moment of the neutron," Phys. Rev. Lett. 97 (2006) 131801, arXiv:hep-ex/0602020 Lhep-ex]. B]

[101] J. E. Kim, "Light pseudoscalars, particle physics and cosmology," Physics Reports 150 no. 1, (1987) 1 - 177. http: //wWw.sciencedirect.com/science/article/pii/0370157387900172. 38

[102] J. E. Kim, "Weak-interaction singlet and strong CP invariance,". 38 


\section{REFERENCES}

[103] M. A. Shifman, A. I. Vainshtein, and V. I. Zakharov, "Can confinement ensure natural CP invariance of strong interactions?," Nuclear Physics B 166 no. 3, (Apr., 1980) 493-506. 38

[104] M. Dine, W. Fischler, and M. Srednicki, "A simple solution to the strong CP problem with a harmless axion," Physics Letters B 104 no. 3, (Aug., 1981) 199-202. 38

[105] A. R. Zhitnitskij, "On possible suppression of the axion-hadron interactions,". http://inis.iaea.org/search/search.aspx?orig_q=RN:11563932. 38

[106] J. Preskill, M. B. Wise, and F. Wilczek, "Cosmology of the Invisible Axion," Phys. Lett. B120 (1983) 127-132. [,URL(1982)]. [8, ए32]

[107] L. F. Abbott and P. Sikivie, "A Cosmological Bound on the Invisible Axion," Phys. Lett. B120 (1983) 133-136. [,URL(1982)].

[108] M. Dine and W. Fischler, "The Not So Harmless Axion," Phys. Lett. B120 (1983) 137-141. [,URL(1982)]. B8, ए32

[109] M. B. Green, J. H. Schwarz, and E. Witten, SUPERSTRING THEORY. VOL. 1: INTRODUCTION. Cambridge Monographs on Mathematical Physics. 1988. http://www.cambridge.org/us/academic/subjects/ physics/theoretical-physics-and-mathematical-physics/ superstring-theory-volume-1. [3.

[110] M. B. Green, J. H. Schwarz, and E. Witten, SUPERSTRING THEORY. VOL. 2: LOOP AMPLITUDES, ANOMALIES AND PHENOMENOLOGY. 1988. http://www.cambridge.org/us/academic/ subjects/physics/theoretical-physics-and-mathematical-physics/ superstring-theory-volume-2.

[111] J. Polchinski, String theory. Vol. 1: An introduction to the bosonic string. Cambridge Monographs on Mathematical Physics. Cambridge University Press, 2007. 
[112] J. Polchinski, String Theory: Volume 2, Superstring Theory and Beyond. Cambridge Monographs on Mathematical Physics. Cambridge University Press, 1998. https://books.google.it/books?id=WKatSc5pj0gC.

[113] K. Becker, M. Becker, and J. H. Schwarz, String theory and M-theory: A modern introduction. Cambridge University Press, 2006. B.]

[114] E. Witten, "Some properties of o(32) superstrings," Physics Letters B 149 no. 4, (1984) 351 - 356. http:

//www.sciencedirect.com/science/article/pii/0370269384904222.

उ.9

[115] P. Candelas, G. T. Horowitz, A. Strominger, and E. Witten, "Vacuum configurations for superstrings," Nuclear Physics B 258 (1985) 46 - 74. http:

//wWw.sciencedirect.com/science/article/pii/0550321385906029.

[3.]

[116] A. H. Guth, "The Inflationary Universe: A Possible Solution to the Horizon and Flatness Problems," Phys. Rev. D23 (1981) 347-356. [Adv. Ser. Astrophys. Cosmol.3,139(1987)]. [3.

[117] A. Linde, "A new inflationary universe scenario: A possible solution of the horizon, flatness, homogeneity, isotropy and primordial monopole problems," Physics Letters B 108 no. 6, (1982) 389 - 393. http: //wWw.sciencedirect.com/science/article/pii/0370269382912199.

[118] D. Baumann, "Inflation,"] in Physics of the large and the small, TASI 09, proceedings of the Theoretical Advanced Study Institute in Elementary Particle Physics, Boulder, Colorado, USA, 1-26 June 2009, pp. 523-686. 2011. arXiv:0907.5424 [hep-th]. उप]

[119] A. Arvanitaki, M. Baryakhtar, and X. Huang, "Discovering the QCD Axion with Black Holes and Gravitational Waves," Phys. Rev. D91 no. 8, (2015) 084011, arXiv:1411.2263 [hep-ph]. 40, ㅁ29, ए3: 


\section{REFERENCES}

[120] A. Arvanitaki, M. Baryakhtar, S. Dimopoulos, S. Dubovsky, and R. Lasenby, "Black Hole Mergers and the QCD Axion at Advanced LIGO," Phys. Rev. D95 no. 4, (2017) 043001, arXiv:1604.03958 [hep-ph]. 40, एवप

[121] A. Arvanitaki and S. Dubovsky, "Exploring the String Axiverse with Precision Black Hole Physics," Phys. Rev. D83 (2011) 044026, arXiv:1004.3558 [hep-th]. 401, 46, ए22., ए32

[122] R. Brito, S. Ghosh, E. Barausse, E. Berti, V. Cardoso, I. Dvorkin, A. Klein, and P. Pani, "Gravitational wave searches for ultralight bosons with LIGO and LISA," Phys. Rev. D96 no. 6, (2017) 064050, arXiv:1706.06311 [gr-qc]. 40]

[123] M. Gross and S. Haroche, "Superradiance: An essay on the theory of collective spontaneous emission," Physics Reports 93 no. 5, (1982) $301-$ 396. http:

//www.sciencedirect.com/science/article/pii/0370157382901028. 410

[124] J. D. Bekenstein and M. Schiffer, "The Many faces of superradiance," Phys. Rev. D58 (1998) 064014, arXiv:gr-qc/9803033 Lgr-qc].

[125] R. Brito, V. Cardoso, and P. Pani, "Superradiance," Lect. Notes Phys. 906

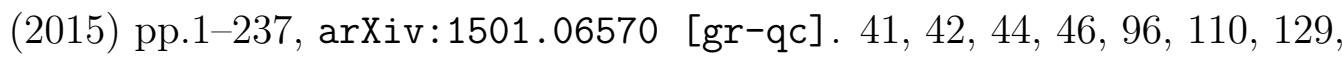
प32

[126] O. Klein, "Die reflexion von elektronen an einem potentialsprung nach der relativistischen dynamik von dirac," Zeitschrift für Physik 53 no. 3, (Mar, 1929) 157-165. https://doi.org/10.1007/BF01339716. 40

[127] V. L. Ginzburg and I. M. Frank 4. , Zh. Éksp. Teor. Fiz. 16, 15 ; J. Phys. (Moscow) 9, 353 (1945) (1946) . 401

[128] R. H. Dicke, "Coherence in spontaneous radiation processes," Phys. Rev. 93 (Jan, 1954) 99-110. https://link.aps.org/doi/10.1103/PhysRev.93.99. 41] 
[129] Y. B. Zel'Dovich, "Generation of Waves by a Rotating Body," Soviet Journal of Experimental and Theoretical Physics Letters 14 (Aug., 1971) 180. 410, 4.3, प6]

[130] K. S. Thorne, R. H. Price, and D. A. MacDonald, Black holes: The membrane paradigm. 1986. 431, ए68, ए69, ए77

[131] W. Press and S. Teukolsky, "Floating orbits, superradiant scattering and the black-hole bomb," Nature 238 no. 5361, (12, 1972) 211-212. 45, 46, 미

[132] V. Cardoso, O. J. C. Dias, J. P. S. Lemos, and S. Yoshida, "The Black hole bomb and superradiant instabilities," Phys. Rev. D70 (2004) 044039, arXiv:hep-th/0404096 Lhep-th]. [Erratum: Phys. Rev.D70,049903(2004)]. 4.9, ए32

[133] S. Hod and O. Hod, "Analytic treatment of the black-hole bomb," Phys. Rev. D81 (2010) 061502, arXiv:0910.0734 [gr-qc]. 15, 46, ए1

[134] C. Palenzuela, T. Garrett, L. Lehner, and S. L. Liebling, "Magnetospheres of Black Hole Systems in Force-Free Plasma," Phys. Rev. D82 (2010) 044045, arXiv:1007.1198 [gr-qc]. 45, 47, ㅁ69

[135] V. Cardoso, I. P. Carucci, P. Pani, and T. P. Sotiriou, "Matter around Kerr black holes in scalar-tensor theories: scalarization and superradiant instability," Phys. Rev. D88 (2013) 044056, arXiv:1305.6936 [gr-qc]. 52, 5.3, 54, 56, 5.9, 미

[136] W. E. East and F. Pretorius, "Superradiant Instability and Backreaction of Massive Vector Fields around Kerr Black Holes," Phys. Rev. Lett. 119 no. 4, (2017) 041101, arXiv:1704.04791 Lgr-qc]. 45

[137] T. Damour, N. Deruelle, and R. Ruffini, "On quantum resonances in stationary geometries," Lettere al Nuovo Cimento (1971-1985) 15 no. 8, (1976) 257-262. https://doi.org/10.1007/BF02725534. 47 
[138] H. Furuhashi and Y. Nambu, "Instability of massive scalar fields in Kerr-Newman space-time," Prog. Theor. Phys. 112 (2004) 983-995, arXiv:gr-qc/0402037 [gr-qc]. 40

[139] V. P. Frolov and I. D. Novikov, eds., Black hole physics: Basic concepts and new developments, vol. 96. 1998. 47, ए68, ए69

[140] M. H. P. M. van Putten, "Superradiance in a Torus Magnetosphere Around a Black Hole," Science 284 no. 5411, (1999) 115-118. 47

[141] S. R. Dolan, S. Ponglertsakul, and E. Winstanley, "Stability of black holes in Einstein-charged scalar field theory in a cavity," Phys. Rev. D92 no. 12, (2015) 124047, arXiv:1507.02156 Lgr-qc]. 47, 49, 501, 50, 58, 미

[142] N. Sanchis-Gual, J. C. Degollado, P. J. Montero, J. A. Font, and C. Herdeiro, "Explosion and Final State of an Unstable

Reissner-Nordström Black Hole," Phys. Rev. Lett. 116 no. 14, (2016) 141101, arXiv: 1512.05358 [gr-qc]. 47, 58

[143] W. Israel, "Event Horizons in Static Vacuum Space-Times," Physical Review 164 no. 5, (Dec, 1967) 1776-1779. 47

[144] R.Ruffini and J.A.Wheeler, "Introducing the black hole," Physics Today 24 no. 30, ((1971)) . 47

[145] H. Luckock and I. Moss, "Black holes have skyrmion hair," Physics Letters B 176 no. 3, (1986) 341 - 345. http:

//www.sciencedirect.com/science/article/pii/0370269386901759. 47

[146] M. S. Volkov and D. V. Galtsov, "NonAbelian Einstein Yang-Mills black holes," JETP Lett. 50 (1989) 346-350. [Pisma Zh. Eksp. Teor.

Fiz.50,312(1989)]. 47

[147] M. S. Volkov and D. V. Galtsov, "Black holes in Einstein Yang-Mills theory.," Sov. J. Nucl. Phys. 51 (1990) 747-753. [Yad. Fiz.51,1171(1990)]. 47 
[148] M. S. Volkov and D. V. Galt'sov, "Gravitating non-abelian solitons and black holes with yang-mills fields," Physics Reports 319 no. 1, (1999) $1-$ 83. http:

//wWw.sciencedirect.com/science/article/pii/S0370157399000101. 47

[149] K.-M. Lee and E. J. Weinberg, "Charge black holes with scalar hair," Phys. Rev. D44 (1991) 3159-3163. ㅍ33, ㅈप, 메, ㅁ45, ㅁ46

[150] B. A. Campbell, N. Kaloper, and K. A. Olive, "Classical hair for Kerr-Newman black holes in string gravity," Physics Letters B 285 no. 3, (1992) 199 - 205. http:

//wWw.sciencedirect.com/science/article/pii/037026939291452F. ए13, ए3.3, ए3., प4य

[151] P. Breitenlohner, P. Forgács, and D. Maison, "Static spherically symmetric solutions of the einstein-yang-mills equations," Communications in Mathematical Physics 163 no. 1, (1994) 141-172. https://doi.org/10.1007/BF02101738.

[152] S. W. Hawking, M. J. Perry, and A. Strominger, "Soft Hair on Black Holes," Phys. Rev. Lett. 116 no. 23, (2016) 231301, arXiv:1601.00921 [hep-th]. 47

[153] J. D. Bekenstein, "Black hole hair: twenty-five years after," 1996. 47

[154] M. S. Volkov, "Hairy black holes in the XX-th and XXI-st centuries," in Proceedings, 14th Marcel Grossmann Meeting on Recent Developments in Theoretical and Experimental General Relativity, Astrophysics, and Relativistic Field Theories (MG14) (In 4 Volumes): Rome, Italy, July 12-18, 2015, vol. 2, pp. 1779-1798. 2017. arXiv:1601.08230 [gr-qc]. 47, $\square 38$

[155] B. A. Campbell, M. J. Duncan, N. Kaloper, and K. A. Olive, "Axion hair for Kerr black holes," Phys. Lett. B251 (1990) 34-38. 48, ㅁ.34, ․3., ㄸ.3, ㄷ.5. 


\section{REFERENCES}

[156] T. P. Sotiriou and S.-Y. Zhou, "Black hole hair in generalized scalar-tensor gravity," Phys. Rev. Lett. 112 (2014) 251102, arXiv:1312.3622 [gr-qc]. [ए.33, , [1.39, ㅁ.46]

[157] C. A. R. Herdeiro and E. Radu, "Kerr black holes with scalar hair," Phys. Rev. Lett. 112 (2014) 221101, arXiv:1403.2757 [gr-qc]. [88, [63, 87

[158] C. A. R. Herdeiro and E. Radu, "Asymptotically flat black holes with scalar hair: a review," Int. J. Mod. Phys. D24 no. 09, (2015) 1542014, arXiv:1504.08209 [gr-qc]. 62, [.38

[159] R. Benkel, T. P. Sotiriou, and H. Witek, "Black hole hair formation in shift-symmetric generalised scalar-tensor gravity," Class. Quant. Grav. 34 no. 6, (2017) 064001, arXiv:1610.09168 [gr-qc].

[160] C. Herdeiro, E. Radu, and H. Rúnarsson, "Kerr black holes with Proca hair," Class. Quant. Grav. 33 no. 15, (2016) 154001, arXiv:1603.02687 [gr-qc].

[161] L. Heisenberg, R. Kase, M. Minamitsuji, and S. Tsujikawa, "Hairy black-hole solutions in generalized Proca theories," Phys. Rev. D96 no. 8 , (2017) 084049, arXiv:1705.09662 [gr-qc]. 62, 67

[162] J. Zhang and H. Yang, "Gravitational floating orbits around hairy black holes," Phys. Rev. D99 no. 6, (2019) 064018, arXiv:1808.02905 [gr-qc]. 487, 다미

[163] N. Yunes and L. C. Stein, "Non-Spinning Black Holes in Alternative Theories of Gravity," Phys. Rev. D83 (2011) 104002, arXiv:1101.2921 $[\mathrm{Lr}-\mathrm{qc}]$. 52

[164] T. P. Sotiriou, "Black Holes and Scalar Fields," Class. Quant. Grav. 32 no. 21, (2015) 214002, arXiv:1505.00248 [gr-qc]. 62, [2], ए38

[165] S. Hod, "Stationary Scalar Clouds Around Rotating Black Holes," Phys. Rev. D86 (2012) 104026, arXiv:1211.3202 Lgr-qc]. [Erratum: Phys.

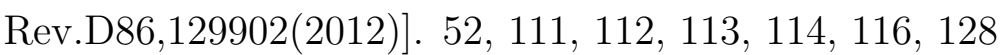


[166] W. F. Buell and B. A. Shadwick, "Potentials and bound states," American Journal of Physics 63 no. 3, (1995) 256-258,

https://doi.org/10.1119/1.17935.

https://doi.org/10.1119/1.17935. 56

[167] D. R. Brill, P. L. Chrzanowski, C. M. Pereira, E. D. Fackerell, and J. R. Ipser, "Solution of the scalar wave equation in a kerr background by separation of variables," Phys. Rev. D 5 (Apr, 1972) 1913-1915. https://link.aps.org/doi/10.1103/PhysRevD.5.1913. 58, ए6

[168] S. Detweiler, "Klein-gordon equation and rotating black holes," Phys. Rev. D 22 (Nov, 1980) 2323-2326. https://link.aps.org/doi/10.1103/PhysRevD.22.2323. 5.9, ШП, ए6, ए32

[169] F. Filippini and G. Tasinato, "An exact solution for a rotating black hole in modified gravity," JCAP 1801 no. 01, (2018) 033, arXiv:1709.02147 [hep-th]. 6], 62

[170] N. Yunes, K. Yagi, and F. Pretorius, "Theoretical Physics Implications of the Binary Black-Hole Mergers GW150914 and GW151226," Phys. Rev. D94 no. 8, (2016) 084002, arXiv:1603.08955 [gr-qc]. 62, ए07

[171] E. J. Copeland, M. Sami, and S. Tsujikawa, "Dynamics of dark energy," Int. J. Mod. Phys. D15 (2006) 1753-1936, arXiv:hep-th/0603057 [hep-th]. 62

[172] A. Joyce, B. Jain, J. Khoury, and M. Trodden, "Beyond the Cosmological Standard Model," Phys. Rept. 568 (2015) 1-98, arXiv:1407.005.9 [astro-ph.CO]. 62

[173] A. Nicolis, R. Rattazzi, and E. Trincherini, "The Galileon as a local modification of gravity," Phys. Rev. D79 (2009) 064036, arXiv:0811.2197 [hep-th]. 62 


\section{REFERENCES}

[174] G. W. Horndeski, "Second-Order Scalar-Tensor Field Equations in a Four-Dimensional Space," International Journal of Theoretical Physics 10 (Sept., 1974) 363-384. 62

[175] B. M. Gripaios, "Modified gravity via spontaneous symmetry breaking," JHEP 10 (2004) 069, arXiv: hep-th/0408127 Lhep-th]. 62, 64

[176] G. Tasinato, "Cosmic Acceleration from Abelian Symmetry Breaking," JHEP 04 (2014) 067, arXiv: 1402.6450 [hep-th]. 62, 64

[177] J. Chagoya, G. Niz, and G. Tasinato, "Black Holes and Abelian Symmetry Breaking," Class. Quant. Grav. 33 no. 17, (2016) 175007, arXiv:1602.08697 [hep-th]. 62, 67

[178] M. Minamitsuji, "Solutions in the generalized Proca theory with the nonminimal coupling to the Einstein tensor," Phys. Rev. D94 no. 8, (2016) (0840:39, arXiv: 1607.06278 [gr-qc].

[179] A. Cisterna, M. Hassaine, J. Oliva, and M. Rinaldi, "Static and rotating solutions for Vector-Galileon theories," Phys. Rev. D94 no. 10, (2016) [114(1):39, arXiv:1609.03430 [gr-qc].

[180] E. Babichev, C. Charmousis, and M. Hassaine, "Black holes and solitons in an extended Proca theory," JHEP 05 (2017) 114, arXiv: 1703.07676 $\lfloor g r-q c]$.

[181] J. Chagoya, G. Niz, and G. Tasinato, "Black Holes and Neutron Stars in Vector Galileons," Class. Quant. Grav. 34 no. 16, (2017) 165002, arXiv:1703.09555 [gr-qc].

[182] L. Heisenberg, R. Kase, M. Minamitsuji, and S. Tsujikawa, "Black holes in vector-tensor theories," JCAP 1708 no. 08, (2017) 024, arxiv:1706.0511b $[\mathrm{gr}-\mathrm{qc}]$.

[183] J. Chagoya and G. Tasinato, "Stealth configurations in vector-tensor theories of gravity," JCAP 1801 no. 01, (2018) 046, arXiv:1707.079b1 [hep-th]. 
[184] S. Nakamura, R. Kase, and S. Tsujikawa, "Suppression of matter couplings with a vector field in generalized Proca theories," Phys. Rev. D96 no. 8, (2017) 084005, arXiv:1707.09194 [gr-qc]. 62, 67

[185] E. Babichev and A. Fabbri, "Rotating black holes in massive gravity," Phys. Rev. D90 (2014) 084019, arXiv:1406.6096 [gr-qc]. 6.3

[186] J. W. Moffat, "Modified Gravity Black Holes and their Observable Shadows," Eur. Phys. J. C75 no. 3, (2015) 130, arXiv:1502.016r7 [gr-qc]. [63, ㅁ.33

[187] P. Pani, C. F. B. Macedo, L. C. B. Crispino, and V. Cardoso, "Slowly rotating black holes in alternative theories of gravity," Phys. Rev. D84 (2011) 087501, arXiv:1109.3996 [gr-qc]. 6.3

[188] B. Kleihaus, J. Kunz, S. Mojica, and E. Radu, "Spinning black holes in Einstein-Gauss-Bonnet-dilaton theory: Nonperturbative solutions," Phys. Rev. D93 no. 4, (2016) 044047, arXiv:1511.05513 [gr-qc]. [63], 87

[189] A. Maselli, H. O. Silva, M. Minamitsuji, and E. Berti, "Slowly rotating black hole solutions in Horndeski gravity," Phys. Rev. D92 no. 10, (2015) 104(1)49, arXiv: 1508.03044 [gr-qc]. 6.3]

[190] T. Johannsen and D. Psaltis, "A Metric for Rapidly Spinning Black Holes Suitable for Strong-Field Tests of the No-Hair Theorem," Phys. Rev. D83 (2011) 124015, arXiv:1105.3191 Lgr-qc]. 6.3

[191] R. Konoplya, L. Rezzolla, and A. Zhidenko, "General parametrization of axisymmetric black holes in metric theories of gravity," Phys. Rev. D93 no. 6, (2016) 064015, arXiv:1602.02378 Lgr-qc]. 63]

[192] M. Zumalacárregui and J. García-Bellido, "Transforming gravity: from derivative couplings to matter to second-order scalar-tensor theories beyond the Horndeski Lagrangian," Phys. Rev. D89 (2014) 064046, arXiv:1308.4685 [gr-qc]. 6.3 


\section{REFERENCES}

[193] G. Koutsoumbas, K. Ntrekis, E. Papantonopoulos, and E. N. Saridakis, "Unification of Dark Matter - Dark Energy in Generalized Galileon Theories," JCAP 1802 (2018) 003, arXiv:1704.08640 [gr-qc]. 64

[194] R. Kimura, A. Naruko, and D. Yoshida, "Extended vector-tensor theories," JCAP 1701 no. 01, (2017) 002, arXiv:1608.07066 Lgr-qc]. 65, 66]

[195] G. Domènech, S. Mukohyama, R. Namba, and V. Papadopoulos, "Vector disformal transformation of generalized Proca theory," Phys. Rev. D 98 no. 6, (2018) 064037, arXiv:1807.06048 [gr-qc]. 6.5

[196] G. Tasinato, "A small cosmological constant from Abelian symmetry breaking," Class. Quant. Grav. 31 (2014) 225004, arXiv:1404.4883 [hep-th]. 67

[197] A. De Felice, L. Heisenberg, R. Kase, S. Tsujikawa, Y.-l. Zhang, and G.-B. Zhao, "Screening fifth forces in generalized Proca theories," Phys. Rev. D93 no. 10, (2016) 104016, arXiv:1602.00371 [gr-qc].

[198] C. M. Nieto and Y. Rodriguez, "Massive Gauge-flation," Mod. Phys. Lett. A31 no. 21, (2016) 1640005, arXiv:1602.07197 [gr-qc].

[199] A. De Felice, L. Heisenberg, R. Kase, S. Mukohyama, S. Tsujikawa, and Y.-l. Zhang, "Effective gravitational couplings for cosmological perturbations in generalized Proca theories," Phys. Rev. D94 no. 4, (2016) (1441)24, arXiv:1605.05066 [gr-qc].

[200] L. Heisenberg, R. Kase, and S. Tsujikawa, "Anisotropic cosmological solutions in massive vector theories," JCAP 1611 no. 11, (2016) 008, arXiv:1607.03175 Lgr-qc].

[201] S. Nakamura, R. Kase, and S. Tsujikawa, "Cosmology in beyond-generalized Proca theories," Phys. Rev. D95 no. 10, (2017) 104001, arXiv:1702.08610 [gr-qc]. 
[202] A. de Felice, L. Heisenberg, and S. Tsujikawa, "Observational constraints on generalized Proca theories," Phys. Rev. D95 no. 12, (2017) 123540, arXiv:1703.09573 Lastro-ph.CO]. 67

[203] N. Khosravi, "Spontaneous scalar-vector Galileons from a Weyl biconnection model," Phys. Rev. D89 no. 12, (2014) 124027, arXiv:1404.7503 [hep-th]. 67

[204] P. Fleury, J. P. Beltran Almeida, C. Pitrou, and J.-P. Uzan, "On the stability and causality of scalar-vector theories," JCAP 1411 no. 11, (2014) 043, arXiv: 1406.6254 [hep-th].

[205] M. Hull, K. Koyama, and G. Tasinato, "A Higgs Mechanism for Vector Galileons," JHEP 03 (2015) 154, arXiv:1408.6871 Lhep-th].

[206] M. Hull, K. Koyama, and G. Tasinato, "Covariantized vector Galileons," Phys. Rev. D93 no. 6, (2016) 064012, arXiv:1510.07029 [hep-th].

[207] F. Charmchi, Z. Haghani, S. Shahidi, and L. Shahkarami, "One-loop corrections to vector Galileon theory," Phys. Rev. D93 no. 12, (2016) [241444, arXiv: 1511.07034 [hep-th].

[208] J. Chagoya and G. Tasinato, "Galileon Higgs Vortices," JHEP 02 (2016) 063, arXiv:1511.07805 [hep-th].

[209] L. Heisenberg, R. Kase, and S. Tsujikawa, "Beyond generalized Proca theories," Phys. Lett. B760 (2016) 617-626, arXiv:1605.05565 [hep-th].

[210] A. Amado, Z. Haghani, A. Mohammadi, and S. Shahidi, "Quantum corrections to the generalized Proca theory via a matter field," Phys. Lett. B772 (2017) 141-151, arXiv:1612.06938 [hep-th].

[211] J. Bonifacio, K. Hinterbichler, and R. A. Rosen, "Positivity constraints for pseudolinear massive spin-2 and vector Galileons," Phys. Rev. D94 no. 10, (2016) 104001, arXiv:1607.06084 [hep-th]. 
[212] E. Allys, P. Peter, and Y. Rodriguez, "Generalized Proca action for an Abelian vector field," JCAP 1602 no. 02, (2016) 004, arXiv:1511.03101 [hep-th].

[213] J. Beltran Jimenez and L. Heisenberg, "Derivative self-interactions for a massive vector field," Phys. Lett. B757 (2016) 405-411,

arXiv:1602.03410 [hep-th]. 67

[214] J. M. M. Garcia, "http://www.exact.es.". [76]

[215] R. C. Myers and M. J. Perry, "Black holes in higher dimensional space-times," Annals of Physics 172 (Dec., 1986) 304-347. 87

[216] J. M. Bardeen, W. H. Press, and S. A. Teukolsky, "Rotating Black Holes: Locally Nonrotating Frames, Energy Extraction, and Scalar Synchrotron Radiation," apj 178 (Dec., 1972) 347-370. 8.

[217] R. D. Blandford and R. L. Znajek, "Electromagnetic extractions of energy from Kerr black holes," Mon. Not. Roy. Astron. Soc. 179 (1977) 433-456. प01,

[218] M. Bhat, S. Dhurandhar, and N. Dadhich, "Energetics of the Kerr-Newman black hole by the Penrose process," Journal of Astrophysics and Astronomy 6 (June, 1985) 85-100. 96

[219] M. Banados, J. Silk, and S. M. West, "Kerr Black Holes as Particle Accelerators to Arbitrarily High Energy," Phys. Rev. Lett. 103 (2009) III102, arXiv:0909.0169 Lhep-ph]. 96

[220] B. Carter, "Global structure of the kerr family of gravitational fields," Phys. Rev. 174 (Oct, 1968) 1559-1571. https://link.aps.org/doi/10.1103/PhysRev.174.1559. ए03, ए4]

[221] J. M. Bardeen, "Timelike and null geodesics in the Kerr metric.," in Black Holes (Les Astres Occlus), C. Dewitt and B. S. Dewitt, eds., pp. 215-239. 1973. ㅁ.:3 
[222] H. Falcke, F. Melia, and E. Agol, "Viewing the Shadow of the Black Hole at the Galactic Center," apjl 528 (Jan., 2000) L13-L16, astro-ph/9912263. ए10:3

[223] H.-M. Wang, Y.-M. Xu, and S.-W. Wei, "Shadows of Kerr-like black holes in a modified gravity theory," JCAP 1903 no. 03, (2019) 046, arXiv:1810.12767 Lgr-qc]. [0.3

[224] T. Vetsov, G. Gyulchev, and S. Yazadjiev, "Shadows of Black Holes in Vector-Tensor Galileons Modified Gravity," arXiv:1801.04592 [gr-qc]. ए10.3

[225] S. Ajith, A. Saffer, and K. Yagi, "Rotating black holes in valid vector-tensor theories after gw170817," 2020. ए03

[226] T. Anson, E. Babichev, C. Charmousis, and M. Hassaine, "Disforming the kerr metric," 2020. एप]

[227] J. Ben Achour, H. Liu, H. Motohashi, S. Mukohyama, and K. Noui, "On Rotating Black Holes in DHOST Theories," arXiv:2006.07245 [gr-qc]. [0.3], [219]

[228] S. Hod, "Kerr-Newman black holes with stationary charged scalar clouds," Phys. Rev. D90 no. 2, (2014) 024051, arXiv:1406.1179 [gr-qc]. ए07, ए.3, ए4, ए21, ए28

[229] E. Berti, K. Yagi, and N. Yunes, "Extreme Gravity Tests with Gravitational Waves from Compact Binary Coalescences: (I) Inspiral-Merger," Gen. Rel. Grav. 50 no. 4, (2018) 46, arXiv:1801.03208 [gr-qc]. ए08

[230] E. Berti, K. Yagi, H. Yang, and N. Yunes, "Extreme Gravity Tests with Gravitational Waves from Compact Binary Coalescences: (II) Ringdown," Gen. Rel. Grav. 50 no. 5, (2018) 49, arXiv:1801.03587 [gr-qc]. एण8 
[231] C. M. Will, "The Confrontation between General Relativity and Experiment," Living Rev. Rel. 17 (2014) 4, arXiv:1403.7377 [gr-qc]. ए18

[232] N. Yunes and X. Siemens, "Gravitational-Wave Tests of General Relativity with Ground-Based Detectors and Pulsar Timing-Arrays," Living Rev. Rel. 16 (2013) 9, arXiv:1304.3473 [gr-qc].

[233] J. R. Gair, M. Vallisneri, S. L. Larson, and J. G. Baker, "Testing General Relativity with Low-Frequency, Space-Based Gravitational-Wave Detectors," Living Rev. Rel. 16 (2013) 7, arXiv:1212.5575 [gr-qc]. एण8

[234] LISA Collaboration, H. Audley et al., "Laser Interferometer Space Antenna," arXiv:1702.00786 [astro-ph. IM]. ए08]

[235] K. Kuroda, M. OHASHI, S. MIYOKI, D. TATSUMI, S. SATO, H. ISHIZUKA, M.-K. Fujimoto, S. KAWAMURA, R. Takahashi, T. YAMAZAKI, K. ARAI, M. FUKUSHIMA, K. WASEDA, S. TELADA, A. UEDA, T. Shintomi, A. Yamamoto, T. Suzuki, Y. Saito, and M. E. Tobar, "Large-scale cryogenic gravitational wave telescope," International Journal of Modern Physics D 8 (01, 2005) 557-579. ए08

[236] KAGRA Collaboration, K. Somiya, "Detector configuration of KAGRA: The Japanese cryogenic gravitational-wave detector," Class. Quant. Grav. 29 (2012) 124007, arXiv:1111.7185 [gr-qc].

[237] KAGRA Collaboration, T. Akutsu et al., "Construction of KAGRA: an Underground Gravitational Wave Observatory," PTEP 2018 no. 1, (2018)

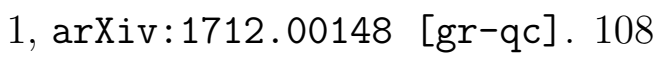

[238] S. Kroker and R. Nawrodt, "The einstein telescope," IFiFi Instrumentation Measurement Magazine 18 no. 3, (June, 2015) 4-8. ए08

[239] B. Sathyaprakash et al., "Scientific Objectives of Einstein Telescope," Class. Quant. Grav. 29 (2012) 124013, arXiv:1206.0331 [gr-qc]. [Erratum: Class. Quant. Grav.30,079501(2013)]. ए08 
[240] LIGO Scientific Collaboration, J. Aasi et al., "Advanced LIGO," Clas.s. Quant. Grav. 32 (2015) 074001, arXiv:1411.4547 [gr-qc]. एuा

[241] VIRGO Collaboration, F. Acernese et al., "Advanced Virgo: a second-generation interferometric gravitational wave detector," Clas.s. Quant. Grav. 32 no. 2, (2015) 024001, arXiv:1408.3978 Lgr-qc]. 미

[242] E. Burns et al., "A Summary of Multimessenger Science with Neutron Star Mergers," arXiv:1903.03582 Lastro-ph.HE]. 미

[243] K. Koyama, "Cosmological Tests of Modified Gravity," Rept. Prog. Phys. 79 no. 4, (2016) 046902, arXiv:1504.04623 [astro-ph.C0]. एण8

[244] Y. Fujii and K.-i. Maeda, The Scalar-Tensor Theory of Gravitation. Cambridge Monographs on Mathematical Physics. Cambridge University Press, 2003. ए0.

[245] S. M. Carroll, "Quintessence and the rest of the world: Suppressing long-range interactions," Phys. Rev. Lett. 81 (Oct, 1998) 3067-3070. https://link.aps.org/doi/10.1103/PhysRevLett.81.3067. [00.9, ㅁ32, प144

[246] T. Damour, "'The Entropy of black holes: A Primer,"] 2004. arXiv:hep-th/0401160 [hep-th]. [0.9]

[247] S. R. Dolan, "Instability of the massive Klein-Gordon field on the Kerr spacetime," Phys. Rev. D76 (2007) 084001, arXiv:0705.2880 [gr-qc].

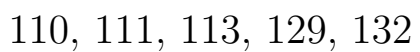

[248] V. Cardoso, I. P. Carucci, P. Pani, and T. P. Sotiriou, "Black holes with surrounding matter in scalar-tensor theories," Phys. Rev. Lett. 111 (2013) ШIII), arXiv:1308.6587 [gr-qc]. ㅁ]

[249] J. Barranco, A. Bernal, J. C. Degollado, A. Diez-Tejedor, M. Megevand, M. Alcubierre, D. Nunez, and O. Sarbach, "Schwarzschild black holes can wear scalar wigs," Phys. Rev. Lett. 109 (2012) 081102, arXiv:1207.2153

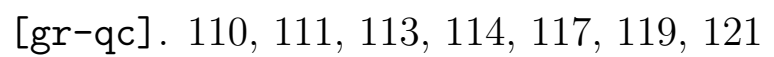


[250] S. Hod, "Stationary resonances of rapidly-rotating Kerr black holes," Eur. Phys. J. C73 no. 4, (2013) 2378, arXiv:1311.5298 [gr-qc]. ШШ, Ш2, ए3, ए4, ए28

[251] S. A. Teukolsky, "Rotating black holes: Separable wave equations for gravitational and electromagnetic perturbations," Phys. Rev. Lett. 29 (Oct, 1972) 1114-1118.

https://link.aps.org/doi/10.1103/PhysRevLett.29.1114. ШШ, Ш4, ए16, ए.32, ए81]

[252] G. Cheng, W. Lin, and R. Hsu, "Dyonic black holes in dilaton gravity," Journal of Mathematical Physics 35 no. 9, (1994) 4839-4847,

https://doi.org/10.1063/1.530817. https://doi.org/10.1063/1.530817. Ш०3

[253] N. Arkani-Hamed and N. Weiner, "LHC Signals for a SuperUnified Theory of Dark Matter," JHEP 12 (2008) 104, arXiv:0810.0714 [hep-ph]. Ш3]

[254] M. Zumalacarregui and U. Seljak, "Limits on stellar-mass compact objects as dark matter from gravitational lensing of type Ia supernovae," Phys. Rev. Lett. 121 no. 14, (2018) 141101, arXiv:1712.02240 [astro-ph.C0]. एँ3

[255] W. Press and S. A. Teukolsky, "Perturbations of a rotating black hole. ii. dynamical stability of the kerr metric," The Astrophysical Journal 185 (09, 1973) 649-674. ㅍ, ए6

[256] I. A. S. M. Abramowitz, Handbook of Mathematical Functions with Formulas, Graphs, and Mathematical Tables. Dover Printing, Dover, New York, 1964. ए6, ए22, ए2:3, ए24, ए27

[257] A. Ronveux, Heun's Differential Equations. Oxford university Press, Oxford, 1995.

[258] C. Flammer, Spheroidal Wave Functions. Stanford University Press, Stanford, 1956. 미 
[259] Handbook of Differential Equations. MA: Academic Press, Boston, 1997. $\square 27$

[260] G. Carosi, A. Friedland, M. Giannotti, M. J. Pivovaroff, J. Ruz, and J. K. Vogel, "Probing the axion-photon coupling: phenomenological and experimental perspectives. A snowmass white paper," in Proceedings, 2013 Community Summer Study on the Future of U.S. Particle Physics: Snowmass on the Mississippi (CSS2013): Minneapolis, MN, USA, July 29-August 6, 2013. 2013. arXiv:1309.7035 Lhep-ph]. http://www.slac.stanford.edu/econf/C1307292/docs/ submittedArxivFiles/1309.7035.pdf. ए29

[261] A. D. Plascencia and A. Urbano, "Black hole superradiance and polarization-dependent bending of light," JCAP 1804 no. 04, (2018) 059,

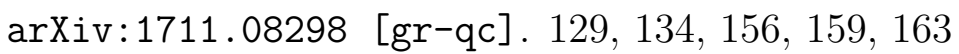

[262] B. Garbrecht and J. I. McDonald, "Axion configurations around pulsars," JCAP 1807 no. 07, (2018) 044, arXiv:1804.04224 Lastro-ph.C0]. [24, ए32

[263] S. Hod, "Charged reflecting stars supporting charged massive scalar field configurations," Eur. Phys. J. C78 no. 3, (2018) 173, arXiv:1801.02801 [hep-th]. [2.9

[264] M. C. Ferreira, C. F. B. Macedo, and V. Cardoso, "Orbital fingerprints of ultralight scalar fields around black holes," Phys. Rev. D96 no. 8, (2017) [883017, arXiv:1710.00830 [gr-qc]. ए2.9]

[265] D. Baumann, H. S. Chia, and R. A. Porto, "Probing Ultralight Bosons with Binary Black Holes," Phys. Rev. D99 no. 4, (2019) 044001, arXiv:1804.03208 [gr-qc]. ए29]

[266] E. Berti, R. Brito, C. F. B. Macedo, G. Raposo, and J. L. Rosa, "Ultralight boson cloud depletion in binary systems," arXiv:1904.03131 Lgr-qc]. एवप 


\section{REFERENCES}

[267] H. Yoshino and H. Kodama, "Gravitational radiation from an axion cloud around a black hole: Superradiant phase," PTEP 2014 (2014) 043E02, arXiv:1312.2326 Lgr-qc]. ए29

[268] R. Brito, S. Ghosh, E. Barausse, E. Berti, V. Cardoso, I. Dvorkin, A. Klein, and P. Pani, "Stochastic and resolvable gravitational waves from ultralight bosons," Phys. Rev. Lett. 119 no. 13, (2017) 131101, arXiv:1706.05097 [gr-qc]. [ए:2]

[269] L. Tsukada, T. Callister, A. Matas, and P. Meyers, "A first search for a stochastic gravitational-wave background from ultralight bosons," arXiv:1812.09622 Lastro-ph.HE]. ए29

[270] F. Filippini and G. Tasinato, "On long range axion hairs for black holes," Class. Quant. Grav. 36 no. 21, (2019) 215015, arXiv:1903.02950 [gr-qc]. ए3], ए32

[271] S. Weinberg, "A New Light Boson?," Phys. Rev. Lett. 40 (1978) 223-226. ए.3.

[272] A. Hook, "TASI Lectures on the Strong CP Problem and Axions," arXiv:1812.02669 [hep-ph]. [332, [333

[273] M. S. Turner, "Coherent Scalar Field Oscillations in an Expanding Universe," Phys. Rev. D28 (1983) 1243. ㅍ.32

[274] W. H. Press, B. S. Ryden, and D. N. Spergel, "Single Mechanism for Generating Large Scale Structure and Providing Dark Missing Matter," Phys. Rev. Lett. 64 (1990) 1084.

[275] S.-J. Sin, "Late time cosmological phase transition and galactic halo as Bose liquid," Phys. Rev. D50 (1994) 3650-3654, arXiv:hep-ph/9205208 [hep-ph].

[276] W. Hu, R. Barkana, and A. Gruzinov, "Cold and fuzzy dark matter," Phys. Rev. Lett. 85 (2000) 1158-1161, arXiv:astro-ph/0003365 [astro-ph]. 
[277] J. Goodman, "Repulsive dark matter," New Astron. 5 (2000) 103, arXiv:astro-ph/0003018 [astro-ph].

[278] P. J. E. Peebles, "Fluid dark matter," Astrophys. J. 534 (2000) L127, arXiv:astro-ph/0002495 [astro-ph].

[279] L. Amendola and R. Barbieri, "Dark matter from an ultra-light pseudo-Goldsone-boson," Phys. Lett. B642 (2006) 192-196, arXiv:hep-ph/0509257 [hep-ph].

[280] H.-Y. Schive, T. Chiueh, and T. Broadhurst, "Cosmic Structure as the Quantum Interference of a Coherent Dark Wave," Nature Phys. 10 (2014) 496-49.9, arXiv:1406.6586 Lastro-ph.GA].

[281] L. Hui, J. P. Ostriker, S. Tremaine, and E. Witten, "Ultralight scalars as cosmological dark matter," Phys. Rev. D95 no. 4, (2017) 043541, arXiv:1610.08297 Lastro-ph.C0]. [32]

[282] V. Cardoso and S. Yoshida, "Superradiant instabilities of rotating black branes and strings," JHEP 07 (2005) 009, arXiv:hep-th/0502206 [hep-th]. [32

[283] A. Hook and J. Huang, "Probing axions with neutron star inspirals and other stellar processes," JHEP 06 (2018) 036, arXiv: 1708.08464 [hep-ph]. [32

[284] J. Huang, M. C. Johnson, L. Sagunski, M. Sakellariadou, and J. Zhang, "Prospects for axion searches with Advanced LIGO through binary mergers," arXiv:1807.02133 Lhep-ph]. [132

[285] J. D. Bekenstein, "Nonexistence of baryon number for static black holes," Phys. Rev. D5 (1972) 1239-1246. ए32, ए38

[286] J. D. Bekenstein, "Novel no-scalar-hair theorem for black holes," Phys. Rev. D51 no. 12, (1995) R6608. ㅍ.2, ए38 


\section{REFERENCES}

[287] T. P. Sotiriou and S.-Y. Zhou, "Black hole hair in generalized scalar-tensor gravity: An explicit example," Phys. Rev. D90 (2014) 124063, arXiv:1408.1698 [gr-qc]. [3:3, ए446]

[288] L. Hui and A. Nicolis, "No-Hair Theorem for the Galileon," Phys. Rev.

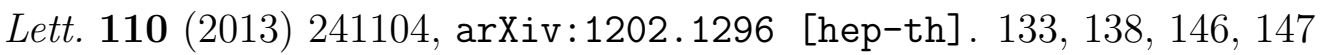

[289] B. A. Campbell, N. Kaloper, and K. A. Olive, "Axion hair for dyon black

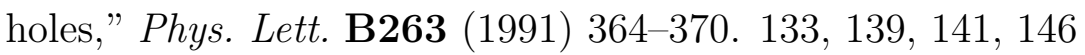

[290] M. Boskovic, R. Brito, V. Cardoso, T. Ikeda, and H. Witek, "Axionic instabilities and new black hole solutions," Phys. Rev. D99 no. 3, (2019) [335006, arXiv: 1811.04945 Lgr-qc]. [34, ए55

[291] A. B. Balakin and W.-T. Ni, "Non-minimal coupling of photons and axions," Class. Quant. Grav. 27 (2010) 055003, arxiv:0911.2946 [gr-qc]. [36]

[292] S. Folkerts, C. Germani, and J. Redondo, "Axion Dark Matter and Planck favor non-minimal couplings to gravity," Phys. Lett. B728 (2014) 532-536, arXiv:1304.7270 [hep-ph]. ए37

[293] R. R. Metsaev and A. A. Tseytlin, "Order alpha-prime (Two Loop) Equivalence of the String Equations of Motion and the Sigma Model Weyl Invariance Conditions: Dependence on the Dilaton and the Antisymmetric Tensor," Nucl. Phys. B293 (1987) 385-419. ए37

[294] A. A. Starobinsky, "A New Type of Isotropic Cosmological Models Without Singularity," Phys. Lett. B91 (1980) 99-102. [,771(1980)]. ए37

[295] F. L. Bezrukov and M. Shaposhnikov, "The Standard Model Higgs boson as the inflaton," Phys. Lett. B659 (2008) 703-706, arXiv:0710.3755 [hep-th]. [137

[296] C. Germani and A. Kehagias, "New Model of Inflation with Non-minimal Derivative Coupling of Standard Model Higgs Boson to Gravity," Phys. Rev. Lett. 105 (2010) 011302, arXiv:1003.2635 [hep-ph]. ए37 
[297] M. Reuter, "A Mechanism generating axion hair for Kerr black holes," Class. Quant. Grav. 9 (1992) 751-756. ㅍप, 띠

[298] A. B. Balakin and A. E. Zayats, "Einstein-Maxwell-axion theory: dyon solution with regular electric field," Eur. Phys. J. C77 no. 8, (2017) 519, arXiv:1703.08858 Lgr-qc].

[299] A. B. Balakin and A. E. Zayats, "Nonminimal black holes with regular electric field," Int. J. Mod. Phys. D24 no. 09, (2015) 1542009, arXiv:1506.05236 [gr-qc].

[300] A. B. Balakin, J. P. S. Lemos, and A. E. Zayats, "Regular nonminimal magnetic black holes in spacetimes with a cosmological constant," Phys. Rev. D93 no. 2, (2016) 024008, arXiv:1512.02653 [gr-qc]. [3.

[301] J. L. Feng, M. Kaplinghat, H. Tu, and H.-B. Yu, "Hidden Charged Dark Matter," JCAP 0907 (2009) 004, arXiv:0905.3039 [hep-ph]. [4]

[302] P. Agrawal, F.-Y. Cyr-Racine, L. Randall, and J. Scholtz, "Make Dark Matter Charged Again," JCAP 1705 no. 05, (2017) 022, arXiv:1610.04611 Lhep-ph]. [40]

[303] G. W. Gibbons and K.-i. Maeda, "Black Holes and Membranes in Higher Dimensional Theories with Dilaton Fields," Nucl. Phys. B298 (1988) $741-775$. 매]

[304] A. D. Shapere, S. Trivedi, and F. Wilczek, "Dual dilaton dyons," Mod. Phys. Lett. A6 (1991) 2677-2686. 40

[305] T. Ortin, Gravity and Strings. Cambridge Monographs on Mathematical Physics. Cambridge University Press, 2015. http://www.cambridge.org/mw/academic/subjects/physics/ theoretical-physics-and-mathematical-physics/ gravity-and-strings-2nd-edition. 40 


\section{REFERENCES}

[306] E. G. Adelberger, B. R. Heckel, and A. E. Nelson, "Tests of the gravitational inverse square law," Ann. Rev. Nucl. Part. Sci. 53 (2003) [77-121, arXiv:hep-ph/0307284 Lhep-ph]. ए44

[307] E. Witten, "Dyons of Charge e theta/2 pi," Phys. Lett. B86 (1979) 283-287. [,283(1979)]. 145

[308] F. Wilczek, "Two Applications of Axion Electrodynamics," Phys. Rev. Lett. 58 (1987) 1799. ㄸ45

[309] M. Rinaldi, "Black holes with non-minimal derivative coupling," Phys. Rev. D86 (2012) 084048, arXiv:1208.0103 [gr-qc]. 147

[310] A. Anabalon, A. Cisterna, and J. Oliva, "Asymptotically locally AdS and flat black holes in Horndeski theory," Phys. Rev. D89 (2014) 084050, arXiv:1312.3597 [gr-qc].

[311] M. Minamitsuji, "Solutions in the scalar-tensor theory with nonminimal derivative coupling," Phys. Rev. D89 (2014) 064017, arxiv:1312.3759 [gr-qc]. 147

[312] "Asymptotically flat black holes in Horndeski theory and beyond," ICAP 1704 no. 04, (2017) 027, arXiv:1702.01938 Lgr-qc]. $\square 47$

[313] T. Jacobson, G. Kang, and R. C. Myers, "On black hole entropy," Phys. Rev. D49 (1994) 6587-6598, arXiv:gr-qc/9312023 [gr-qc]. 147

[314] G. Clément and D. Gal'tsov, "On the Smarr formula for rotating dyonic black holes," Phys. Lett. B773 (2017) 290-294, arXiv:170\%.01332 [gr-qc]. 148

[315] V. S. Manko and H. García-Compeán, "Smarr formula for black holes endowed with both electric and magnetic charges," Class. Quant. Grav. 35 no. 6, (2018) 064001, arXiv:1506.03870 Lgr-qc]. 148

[316] G. W. Gibbons, R. Kallosh, and B. Kol, "Moduli, scalar charges, and the first law of black hole thermodynamics," Phys. Rev. Lett. 77 (1996) 4992-4995, arXiv:hep-th/9607108 [hep-th]. [44] 
[317] K. Konno, T. Matsuyama, and S. Tanda, "Rotating black hole in extended Chern-Simons modified gravity," Prog. Theor. Phys. 122 (2009) 561-568, arXiv:0902.4767 [gr-qc]. ए5.

[318] M. Cambiaso and L. F. Urrutia, "An extended solution space for Chern-Simons gravity: the slowly rotating Kerr black hole," Phys. Rev. D82 (2010) 101502, arXiv:1010.4526 [gr-qc].

[319] K. Yagi, N. Yunes, and T. Tanaka, "Slowly Rotating Black Holes in Dynamical Chern-Simons Gravity: Deformation Quadratic in the Spin," Phys. Rev. D86 (2012) 044037, arXiv:1206.6130 Lgr-qc]. [Erratum: Phys. Rev.D89,049902(2014)].

[320] L. C. Stein, "Rapidly rotating black holes in dynamical Chern-Simons gravity: Decoupling limit solutions and breakdown," Phys. Rev. D90 no. 4, (2014) 044061, arXiv:1407.2350 [gr-qc].

[321] K. Konno and R. Takahashi, "Scalar field excited around a rapidly rotating black hole in Chern-Simons modified gravity," Phys. Rev. D90 no. 6, (2014) 064011, arXiv:1406.0957 [gr-qc].

[322] "Extremal black holes in dynamical Chern?Simons gravity," Class. Quant. Grav. 33 no. 23, (2016) 235013, arXiv:1512.05453 [gr-qc].

[323] T. Delsate, C. Herdeiro, and E. Radu, "Non-perturbative spinning black holes in dynamical Chern?Simons gravity," Phys. Lett. B787 (2018) 8-15, arXiv:1806.06700 [gr-qc]. 152

[324] R. M. Wald, "Black hole in a uniform magnetic field," Phys. Rev. D10 (1974) 1680-1685. ए52

[325] G. R. Fowles, Introduction to Modern Optics. Dover Publications, New York, 1989. 157

[326] Lobanov, A. P., Gómez, J. L., Bruni, G., Kovalev, Y. Y., Anderson, J., Bach, U., Kraus, A., Zensus, J. A., Lisakov, M. M., Sokolovsky, K. V., and Voytsik, P. A., "Radioastron space vlbi imaging of polarized radio emission 


\section{REFERENCES}

in the high-redshift quasar $0642+449$ at 1.6 ghz," $A \mathscr{E A} 583$ (2015) A100. https://doi.org/10.1051/0004-6361/201526335. [58

[327] RadioAstron Collaboration, N. S. Kardashev and V. V. Khartov, "RadioAstron - a Telescope with a Size of 300000 km: Main Parameters and First Observational Results," Astronomy Reports 57 (2013) 153-194, arXiv:1303.5013 Lastro-ph.IM]. 158

[328] S. M. Carroll, G. B. Field, and R. Jackiw, "Limits on a lorentz- and parity-violating modification of electrodynamics," Phys. Rev. D 41 (Feb, 1990) 1231-1240. https://link.aps.org/doi/10.1103/PhysRevD.41.1231. [5y

[329] V. Beckmann and C. R. Shrader, Active Galactic Nuclei. Aug., 2012. ए68

[330] H. Netzer, The Physics and Evolution of Active Galactic Nuclei. Nov., 2013. ए68

[331] P. Goldreich and W. H. Julian, "Pulsar electrodynamics," Astrophys. J. 157 (1969) 869. ए68

[332] H. Grad and H. Rubin, "Hydromagnetic equilibria and force-free fields," tech. rep., United Nations (UN), 1958.

http://inis.iaea.org/search/search.aspx?orig_q=RN:39082408. ए68

[333] G. E. Marsh, Force-Free Magnetic Fields: Solutions, Topology and Applications. WORLD SCIENTIFIC, 1996. https://www.worldscientific.com/doi/pdf/10.1142/2965. https://wWw.worldscientific.com/doi/abs/10.1142/2965.

[334] I. Contopoulos, D. Kazanas, and D. B. Papadopoulos, "The Force-free Magnetosphere of a Rotating Black Hole," apj 765 no. 2, (2013) 113. ㄸ68, ए69

[335] S. S. Komissarov, "Electrodynamics of black hole magnetospheres," Monthly Notices of the Royal Astronomical Society 350 no. 2, (05, 2004) $427-448$, 


\section{REFERENCES}

http://oup.prod.sis.lan/mnras/article-pdf/350/2/427/3884469/350-2-427.pdf. https://doi.org/10.1111/j.1365-2966.2004.07598.x. ए69]

[336] J. C. McKinney and R. D. Blandford, "Stability of relativistic jets from rotating, accreting black holes via fully three-dimensional magnetohydrodynamic simulations," Monthly Notices of the Royal Astronomical Society: Letters 394 no. 1, (03, 2009) L126-L130, http://oup.prod.sis.lan/mnrasl/article-pdf/394/1/L126/3778390/394-1-L126.pdf. https://doi.org/10.1111/j.1745-3933.2009.00625.x.

[337] A. Nathanail and I. Contopoulos, "Black Hole Magnetospheres," Astrophys. J. 788 no. 2, (2014) 186, arXiv:1404.0549 [astro-ph.HE].

[338] H. Yang, F. Zhang, and L. Lehner, "Magnetosphere of a Kerr black hole immersed in magnetized plasma and its perturbative mode structure," Phys. Rev. D91 no. 12, (2015) 124055, arXiv:1503.06/88 [astro-ph.HE]. [6.]

[339] E. Berti, V. Cardoso, and A. O. Starinets, "Quasinormal modes of black holes and black branes," Class. Quant. Grav. 26 (2009) 163001, arXiv:0905.2975 Lgr-qc]. ए79, ए83

[340] P. Pani, "Advanced Methods in Black-Hole Perturbation Theory," Int. . . Mod. Phys. A28 (2013) 1340018, arXiv:1305.6759 [gr-qc]. ㅁ]

[341] S. Chandrasekhar, The mathematical theory of black holes. 1983. 때, 80, ए98

[342] K. D. Kokkotas and B. G. Schmidt, "Quasinormal modes of stars and black holes," Living Rev. Rel. 2 (1999) 2, arXiv:gr-qc/9909058 Lgr-qc]. ए8], 183], [19.9

[343] H.-P. Nollert, "TOPICAL REVIEW: Quasinormal modes: the characteristic 'sound' of black holes and neutron stars," Class. Quant. Grav. 16 (1999) R159-R216. 머, 미 


\section{REFERENCES}

[344] E. Newman and R. Penrose, "An approach to gravitational radiation by a method of spin coefficients," Journal of Mathematical Physics 3 no. 3, (1962) 566-578, https://doi.org/10.1063/1.1724257. https://doi.org/10.1063/1.1724257. एт.9

[345] F. J. Zerilli, "Effective potential for even-parity regge-wheeler gravitational perturbation equations," Phys. Rev. Lett. 24 (Mar, 1970) 737-738. https://link.aps.org/doi/10.1103/PhysRevLett.24.737. ए79]

[346] F. J. Zerilli, "Gravitational field of a particle falling in a schwarzschild geometry analyzed in tensor harmonics," Phys. Rev. D 2 (Nov, 1970) 2141-2160). https://link.aps.org/doi/10.1103/PhysRevD.2.2141. ए7.

[347] C. V. Vishveshwara, "Stability of the schwarzschild metric," Phys. Rev. D 1 (1970) 2870-2879. 따

[348] W. H. Press, "Long wave trains of gravitational waves from a vibrating black hole," apjl 170 (Dec, 1971) L105. 180

[349] M. Davis, R. Ruffini, W. H. Press, and R. H. Price, "Gravitational radiation from a particle falling radially into a schwarzschild black hole," Phys. Rev. Lett. 27 (Nov, 1971) 1466-1469. https://link.aps.org/doi/10.1103/PhysRevLett.27.1466. 181]

[350] C. J. Goebel, "Comments on the "vibrations" of a black hole.," apjl 172 (Mar, 1972) L95. ए80

[351] F. J. Zerilli, "Perturbation analysis for gravitational and electromagnetic radiation in a reissner-nordström geometry," Phys. Rev. D 9 (Feb, 1974) 860-868. https://link.aps.org/doi/10.1103/PhysRevD .9.860. 80, [प7], [200, ए201]

[352] V. Moncrief, "Gauge-invariant perturbations of reissner-nordström black holes," Phys. Rev. D 12 (Sep, 1975) 1526-1537. https://link.aps.org/doi/10.1103/PhysRevD.12.1526. 미, एप8 
[353] S. Chandrasekhar and S. Detweiler, "The quasi-normal modes of the schwarzschild black hole," Proceedings of the Royal Society of London. Series A, Mathematical and Physical Sciences 344 no. 1639, (1975) 441-452. http://www . jstor.org/stable/78902. ए80, ए98

[354] V. Ferrari and B. Mashhoon, "New approach to the quasinormal modes of a black hole," Phys. Rev. D 30 (Jul, 1984) 295-304. https://link.aps.org/doi/10.1103/PhysRevD.30.295. 미

[355] R. F. Stark and T. Piran, "Gravitational-wave emission from rotating gravitational collapse," Phys. Rev. Lett. 55 (Aug, 1985) 891-894. https://link.aps.org/doi/10.1103/PhysRevLett.55.891. 8]

[356] E. W. Leaver, "An Analytic representation for the quasi normal modes of Kerr black holes," Proc. Roy. Soc. Lond. A402 (1985) 285-298. प8ा

[357] E. W. Leaver, "Spectral decomposition of the perturbation response of the schwarzschild geometry," Phys. Rev. D 34 (Jul, 1986) 384-408. https://link.aps.org/doi/10.1103/PhysRevD.34.384. ए8]

[358] F. Echeverria, "Gravitational-wave measurements of the mass and angular momentum of a black hole," Phys. Rev. D 40 (Nov, 1989) 3194-3203. https://link.aps.org/doi/10.1103/PhysRevD.40.3194. ㅁ]

[359] P. Anninos, D. Hobill, E. Seidel, L. Smarr, and W.-M. Suen, "Collision of two black holes," prl 71 no. 18, (Nov, 1993) 2851-2854, arXiv:gr-qc/9309016 [gr-qc]. 181

[360] E. E. Flanagan and S. A. Hughes, "Measuring gravitational waves from binary black hole coalescences: 1 . Signal-to-noise for inspiral, merger, and ringdown," Phys. Rev. D57 (1998) 4535-4565, arXiv:gr-qc/9701039 [gr-qc].

[361] L. Motl and A. Neitzke, "Asymptotic black hole quasinormal frequencies," Adv. Theor. Math. Phys. 7 no. 2, (2003) 307-330, arXiv:hep-th/0301173 [hep-th]. [8] 


\section{REFERENCES}

[362] H. Kodama and A. Ishibashi, "A Master equation for gravitational perturbations of maximally symmetric black holes in higher dimensions," Prog. Theor. Phys. 110 (2003) 701-722, arXiv:hep-th/0305147 [hep-th]. ए8], पप9!

[363] F. Pretorius, "Evolution of binary black hole spacetimes," Phys. Rev. Lett. 95 (2005) 121101, arXiv:gr-qc/0507014 [gr-qc]. 8]

[364] I. Sachs and S. N. Solodukhin, "Quasi-Normal Modes in Topologically Massive Gravity," JHEP 08 (2008) 003, arXiv:0806.1788 [hep-th]. 182

[365] P. Gonzalez, E. Papantonopoulos, and J. Saavedra, "Chern-Simons black holes: scalar perturbations, mass and area spectrum and greybody factors," JHEP 08 (2010) 050, arXiv:1003.1381 [hep-th]. 18.

[366] J. G. Rosa and S. R. Dolan, "Massive vector fields on the Schwarzschild spacetime: quasi-normal modes and bound states," Phys. Rev. D85 (2012) 044043, arXiv: 1110.4494 [hep-th]. 182

[367] J. W. York, "Dynamical origin of black-hole radiance," Phys. Rev. D 28 (Dec, 1983) 2929-2945. https://link.aps.org/doi/10.1103/PhysRevD.28.2929. 182

[368] S. Hod, "Bohr's correspondence principle and the area spectrum of quantum black holes," Phys. Rev. Lett. 81 (Nov, 1998) 4293-4296. https://link.aps.org/doi/10.1103/PhysRevLett.81.4293. 182

[369] O. Dreyer, "Quasinormal modes, the area spectrum, and black hole entropy," Phys. Rev. Lett. 90 (2003) 081301, arXiv:gr-qc/0211076 [gr-qc]. 182

[370] J. M. Maldacena, "The Large N limit of superconformal field theories and supergravity," Int. J. Theor. Phys. 38 (1999) 1113-1133, arXiv:hep-th/9711200 [hep-th]. [Adv. Theor. Math. Phys.2,231(1998)]. प18. 
[371] D. T. Son and A. O. Starinets, "Minkowski space correlators in AdS / CFT correspondence: Recipe and applications," JHEP 09 (2002) 042, arXiv:hep-th/0205051 [hep-th]. 8:

[372] P. K. Kovtun and A. O. Starinets, "Quasinormal modes and holography," Phys. Rev. D72 (2005) 086009, arXiv: hep-th/0506184 [hep-th].

[373] L. Fidkowski, V. Hubeny, M. Kleban, and S. Shenker, "The Black hole singularity in AdS / CFT," JHEP 02 (2004) 014, arXiv:hep-th/0306170 [hep-th]. 182

[374] P. Kovtun, D. T. Son, and A. O. Starinets, "Viscosity in strongly interacting quantum field theories from black hole physics," Phys. Rev. Lett. 94 (2005) 111601, arXiv:hep-th/0405231 [hep-th]. 182

[375] S. A. Hartnoll, "Lectures on holographic methods for condensed matter physics," Class. Quant. Grav. 26 (2009) 224002, arXiv:0903.3246 [hep-th].

[376] C. P. Herzog, "Lectures on Holographic Superfluidity and Superconductivity," J. Phys. A42 (2009) 343001, arXiv:0904.1975 [hep-th]. 182

[377] M. Maggiore, Gravitational waves: Volume 2: Astrophysics and cosmology. 01, 2018. ए83, ए88, ⒏, ए94, ए95, ए96

[378] V. Cardoso, C. F. B. Macedo, P. Pani, and V. Ferrari, "Black holes and gravitational waves in models of minicharged dark matter," ICAP $\mathbf{1 6 0 5}$ no. 05, (2016) 054, arXiv: 1604.07845 Lhep-ph]. Ш3], ए84, ए99

[379] J. Redondo and A. Ringwald, "Light shining through walls," Contemp. Phys. 52 (2011) 211-236, arXiv:1011.3741 [hep-ph]. 184

[380] R. Foot and S. Vagnozzi, "Dissipative hidden sector dark matter," Phys. Rev. D91 (2015) 023512, arXiv:1409.7174 [hep-ph]. 184 


\section{REFERENCES}

[381] V. Cardoso and J. P. S. Lemos, "Quasinormal modes of schwarzschild-anti-de sitter black holes: Electromagnetic and gravitational perturbations," Physical Review D 64 no. 8, (Sep, 2001). http://dx.doi.org/10.1103/PhysRevD.64.084017. 188

[382] V. Moncrief, "Odd-parity stability of a Reissner-Nordstrom black hole," Phys. Rev. D9 (1974) 2707-2709. पㅛ, ए201, एण0

[383] V. Moncrief, "Stability of reissner-nordström black holes," Phys. Rev. D 10 (Aug, 1974) 1057-1059. https://link.aps.org/doi/10.1103/PhysRevD.10.1057. पप8, ए201

[384] B. Xanthopoulos, "Metric and electromagnetic perturbations of the reissner-nordstrom black hole," Royal Society of London Proceedings Series A $378(09,1981) 73-88$. एप्8

[385] K. D. Kokkotas and B. F. Schutz, "Black-hole normal modes: A wkb approach. iii. the reissner-nordström black hole," Phys. Rev. D 37 (Jun, 1988) 3378-3387. https://link.aps.org/doi/10.1103/PhysRevD.37.3378. एप8

[386] E. W. Leaver, "Quasinormal modes of reissner-nordström black holes," Phys. Rev. D 41 (May, 1990) 2986-2997. https://link.aps.org/doi/10.1103/PhysRevD.41.2986. [999]

[387] N. Andersson and H. Onozawa, "Quasinormal modes of nearly extreme reissner-nordström black holes," Physical Review D 54 no. 12, (Dec, 1996) [47(1)-7475. http://dx.doi.org/10.1103/PhysRevD.54.7470. [प99]

[388] H. Kodama and A. Ishibashi, "Master equations for perturbations of generalized static black holes with charge in higher dimensions," Prog. Theor. Phys. 111 (2004) 29-73, arXiv:hep-th/0308128 [hep-th]. [प9!

[389] J. Natario and R. Schiappa, "On the classification of asymptotic quasinormal frequencies for d-dimensional black holes and quantum gravity," Adv. Theor. Math. Phys. 8 no. 6, (2004) 1001-1131, arXiv:hep-th/0411267 [hep-th]. एप9 
[390] R. Brito and C. Pacilio, "Quasinormal modes of weakly charged Einstein-Maxwell-dilaton black holes," Phys. Rev. D98 no. 10, (2018) [104042, arXiv: 1807.09081 Lgr-qc]. [प99]

[391] Y. S. Myung and D.-C. Zou, "Quasinormal modes of scalarized black holes in the Einstein-Maxwell-Scalar theory," Phys. Lett. B790 (2019) 400-407, arXiv:1812.03604 [gr-qc]. 201]

[392] D.-C. Zou and Y. S. Myung, "Scalarized Charged Black Holes with Scalar Mass Term," arXiv:1909.11859 [gr-qc]. ए200

[393] J. M. Fernández Tío and G. Dotti, "Black hole nonmodal linear stability under odd perturbations: The Reissner-Nordström case," Phys. Rev. 095 no. 12, (2017) 124041, arXiv:1607.00975 [gr-qc]. [200

[394] G. Dotti and J. M. F. Tío, "Black hole nonmodal linear stability: even perturbations of Reissner-Nordström," arXiv:1911.04562 [gr-qc]. [200] 